INEL-95/0590

November 1995

Nonintrustive Sensing and Control for Intelligent Processing and Design of Castings

RECEIVED

JUL 29996

OOTI

D. C. Kunerth

J. Gray

Engineering

Laboratory

$\mid$ 


\section{DISCLAIMER}

This report was prepared as an account of work sponsored by an agency of the United States Government. Neither the United States Government nor any agency thereof, nor any of their employees, makes any warranty, express or implied, or assumes any legal liability or responsibility for the accuracy, completeness. or usefuliness of any information, apparatus, product or process disclosed, or represents that its use would not infringe privately owned rights. References herein to any specific commercial product, process, or senvice by trade name, trademark, manufacturer, or othenvise, does not necessarily constitute or imply its endorsement, recommendation, or favoring by the United States Government or any agency thereol. The views and opinions of authors expressed herein do not necessarily state or reflect those of the United States Government or any agency thereof. 


\title{
Nonintrusive Sensing and Control for Intelligent Processing and Design of Castings
}

\author{
D. C. Kunerth
}

J. Gray

Published November 1995

\section{Idaho National Engineering Laboratory Materials Physics Department \\ Lockheed Idaho Technologies Company \\ Idaho Falls, Idaho 83415}



DISCLAIMER

Portions of this document may be illegible in electronic image products. Images are produced from the best available original document. 



\begin{abstract}
Two technologies, laser ultrasonics (LUT) for detecting inclusions in liquid metals and $\mathrm{X}$-ray stereography (XS) for detecting and locating porosity and inclusions in castings, were evaluated. LUT is a relatively new technology that has the potential to operate in the harsh environment of metal melting, without melt contamination, because of its noncontacting nature. It was shown that LUT can detect and monitor inclusions in liquid metal, but limitations exist that restrict its implementation as well as minimize the advantage gained from its noncontacting nature.

In many applications it is acceptable for castings to have a limited amount of inclusions or porosity if these are not in a critical location. XS can determine the physical location of defects and, when integrated into a real-time radiographic system, is useful for quickly evaluating the quality of castings prior to adding value via finish steps. It was demonstrated that XS can achieve a location accuracy of $0.2 \mathrm{~mm}$ relative to a part surface and can be configured as an inexpensive add-on to existing real-time systems.
\end{abstract}





\section{CONTENTS}

ABSTRACT

INTRODUCTION AND SUMMARY

PART A: LASER ULTRASONIC DETECTION OF INCLUSIONS IN LIQUID METALS

Objective

Introduction

Ultrasonic Detection Methodologies

Pulse/Echo/Backscatter Defect Detection

Forward Scatter Defect Detection

Laser Ultrasonic Measurement System

Experimentation

Solid Samples

Liquid Metal

Technical Issues

Conclusions and Recommendations

References

Appendix A-Ultrasonic Backscatter Data For Cast Iron Samples

Appendix B--Ultrasonic Attenuation Data For Cast Iron Samples

PART B: X-RAY STEREOGRAPHY TO LOCATE POROSITY ON METAL CASTINGS

\section{Figures}

1. Laser ultrasonic measurement system.

2. Ultrasonic backscatter data for cast steel samples.

3. Ultrasonic attenuation data for case steel samples.

4. Ultrasonic scatter data for Sn-Fe samples obtained with piezoelectric-based system.

5. Ultrasonic scatter data for $\mathrm{Sn}$-Fe samples obtained with laser-based system. 
6. Laser ultrasonic data illustrating increased scatter with increased Fe content.

7. Laser ultrasonic attenuation measurements of liquid tin containing Fe powder.

\section{Tables}

1. Casting data on Alloy 1025 samples from Foundry 103

2. Casting data on carbon steel samples from Foundry 111 


\section{Nonintrusive Sensing and Control for Intelligent Processing and Design of Castings}

\section{INTRODUCTION AND SUMMARY}

The objective of this collaborative effort between the American Foundrymen's Society, Inc., the Idaho National Engineering Laboratory, ${ }^{b}$ and Iowa State University ${ }^{c}$ was to evaluate two technologies, laser ultrasonics (LUT) and x-ray stereography (XS), for use in the casting industry as part of the U.S. Department of Energy's Metal Casting Competitiveness Program. Laser ultrasonics was evaluated for detection of inclusions in liquid metals and real-time based x-ray stereography (XS) for detection and location of porosity and inclusions in castings.

Ultrasonics is capable of detecting inclusions in liquid metals. Unfortunately, use of this technology to determine the inclusion content of liquid metals has been hindered by the need of conventional ultrasonic transducers to physically couple to the metal because the harsh environment is detrimental to the transducers and contact has the potential for melt contamination. LUT is a relatively new technology that has the potential to overcome these problems because it is noncontacting. LUT uses lasers for the generation and detection of ultrasonic waves and thus is well suited for remote implementation in harsh environments. The work performed determined that LUT can detect and monitor inclusions in liquid metal, but limitations were found that restrict its implementation as well as minimize the advantage gained from its noncontacting nature.

Castings are currently used for a variety of applications in which a limited amount of inclusions or porosity can be tolerated if these are not located in a critical area, e.g. stress points or surfaces created by machining. Single projection $\mathrm{x}$-ray radiography has been used for years to detect and size such defects, but it is incapable of locating the defects in three dimensions. XS has the ability to determine the physical location of the defects and, when integrated into a real-time radiographic system, is a useful tool for quickly evaluating the quality of castings prior to adding

a. American Foundrymen's Society, Inc., Des Plaines, Illinois 60016

b. D. C. Kunerth, Idaho National Engineering Laboratory, Idaho Falls, Idaho 83415

c. J. Gray, Iowa State University, Center for Nondestructive Testing, Ames, Iowa 50011 
value via finish steps. The work performed demonstrates that XS can achieve_a location accuracy of $0.2 \mathrm{~mm}$ relative to a part surface and can be an inexpensive add-on to existing real-time systems, thus improving the system capabilities.

This report is composed of two sections, Part A: Laser Ultrasonic Detection of Inclusions in Liquid Metals, on research performed by the Idaho National Engineering Laboratory, and Part $B: X$ ray Stereography to Locate Porosity on Metal Castings, on research performed by Iowa State University. 


\title{
PART A: LASER ULTRASONIC DETECTION OF INCLUSIONS IN LIQUID METALS
}

\author{
Dennis C. Kunerth \\ Idaho National Engineering Laboratory
}

\section{Objective}

The objective of this effort is to demonstrate the feasibility of laser ultrasonics to detect and characterize inclusions in liquid metal.

\section{Introduction}

A significant amount of work by others has demonstrated the ability of ultrasonics (UT) to characterize inclusion content in liquid metals using pulse echo detection, attenuation, and velocity measurement techniques. ${ }^{1-7}$ With respect to industrial implementation, the basic weakness of that work is that conventional ultrasonic transducers need to be physically coupled to the test material. The harsh environment typically associated with liquid metals makes coupling very difficult and unreliable, while increasing the potential for melt contamination through contact. Laser ultrasonics has the potential to overcome some of these problems. Standoff laser ultrasonic techniques use lasers for both production and detection of ultrasonic waves, making them totally noncontacting. Although previously applied to liquid metal, 8 laser ultrasonics were untested for the measurement of inclusions. This laboratory study was performed to determine their feasibility and possible limitations.

\section{Ultrasonic Detection Methodologies}

\section{Pulse Echo/Backscatter Defect Detection}

Pulse-echo defect detection techniques depend on the direct backscatter/scatter of acoustic energy from defects. For a single-phase liquid, inclusions and bubbles are the only acoustic reflectors present. Information about individual inclusions can be obtained via scatter data, but is typically limited to reflectors one half the wavelength and greater. Because of the large volume of liquid metal and possible distribution of inclusions, trying to detect and characterize individual inclusions is deemed 
to be impractical. A more useful approach is to use bulk backscatter techniques that measure the cumulative energy scattered back from all discontinuities within the confines of the UT beam. This technique will indicate localized defect concentrations. The expected trend is to see increased backscatter energy with increased inclusion content. The defects that are detectable are typically larger than or equal to one tenth of the acoustic wavelength. The respective wavelengths of a $5 \mathrm{MHz}$ acoustic wave in aluminum $\left(4.67 \mathrm{~mm} / \mu \mathrm{s}\right.$ at $\left.660^{\circ} \mathrm{C}\right)$ and iron $\left(3.93 \mathrm{~mm} / \mu \mathrm{s}\right.$ at $\left.1539^{\circ} \mathrm{C}\right)$ are 0.93 and $0.79 \mathrm{~mm} .{ }^{9}$ This corresponds to potential inclusion detection capabilities of approximately $0.08 \mathrm{~mm}$ and greater.

The advantage of the bulk backscatter method is that it can be a one-sided measurement, not requiring through transmission or a known reflector in the melt at a known distance. Compared to attenuation and velocity measurements, the disadvantage of bulk backscatter measurements is that a high signal-to-noise ratio is required to allow the small backscatter energy to be detected. This will be a problem for current laser detection systems because their sensitivity is lower than that of piezoelectric transducers.

\section{Forward Scatter Defect Detection}

Attenuation and velocity measurement techniques depend on scattering and/or dissipation of the forward propagating wave and are sensitive to temperature and composition as well as inclusions. Attenuation is the loss of energy in the primary ultrasonic wave packet as it propagates through a known distance. Propagation through and/or reflection off interfaces need to be controlled to prevent unknown losses. Attenuation is proportional to inclusion content, i.e. increased inclusion content increases attenuation. Velocity is the measurement of propagation time of the primary ultrasonic wave packet through a known distance in a single material. Increased inclusion content corresponds to decreased velocity.

Similar to the backscatter measurements, these techniques are typically sensitive to discontinuities greater than or equal to one tenth of the acoustic wavelength, and thus have equivalent defect size detection capabilities. The advantage of these techniques compared to bulk backscatter measurements is that lower signal-to-noise ratios can be tolerated. The disadvantage is that through transmission or use of a known reflector at a known depth in the test material for pulse echo measurements is required. In addition, any interfaces in the path of the acoustic wave will need to be 
controlled or, at the minimum, maintained with constant acoustic properties with respect to reflection and transmission.

\section{Laser Ultrasonic Measurement System}

The laser ultrasonic system used for this effort is illustrated in Figure 1. A pulsed Nd/YAG laser having a $10 \mathrm{~ns}$ pulse width was used to generate ultrasonic waves in the test sample. The generation mechanism was in the ablative regime, in which sufficient pulsed laser energy is used and absorbed to physically expel material from the sample surface. The recoil of the surface produces a high amplitude longitudinal acoustic wave that propagates normal to the sample surface. Depending on the sample, transverse (solid samples) and surface (liquid and solid samples) waves are also produced, but these are not important for this application unless they disrupt the signal of interest. Pulsed laser focal spot sizes used for this study ranged from 3 to $6 \mathrm{~mm}$ with pulse energies from 70 to $190 \mathrm{~mJ} /$ pulse. A confocal Fabry-Perot interferometer detected the acoustic wave on the surface of the sample via Doppler shifting of reflected/scattered laser light having a known base frequency. The frequency-shifted signal was demodulated by setting the detection laser base frequency on the edge of one of the response curves of the interferometer to obtain an intensity output proportional to

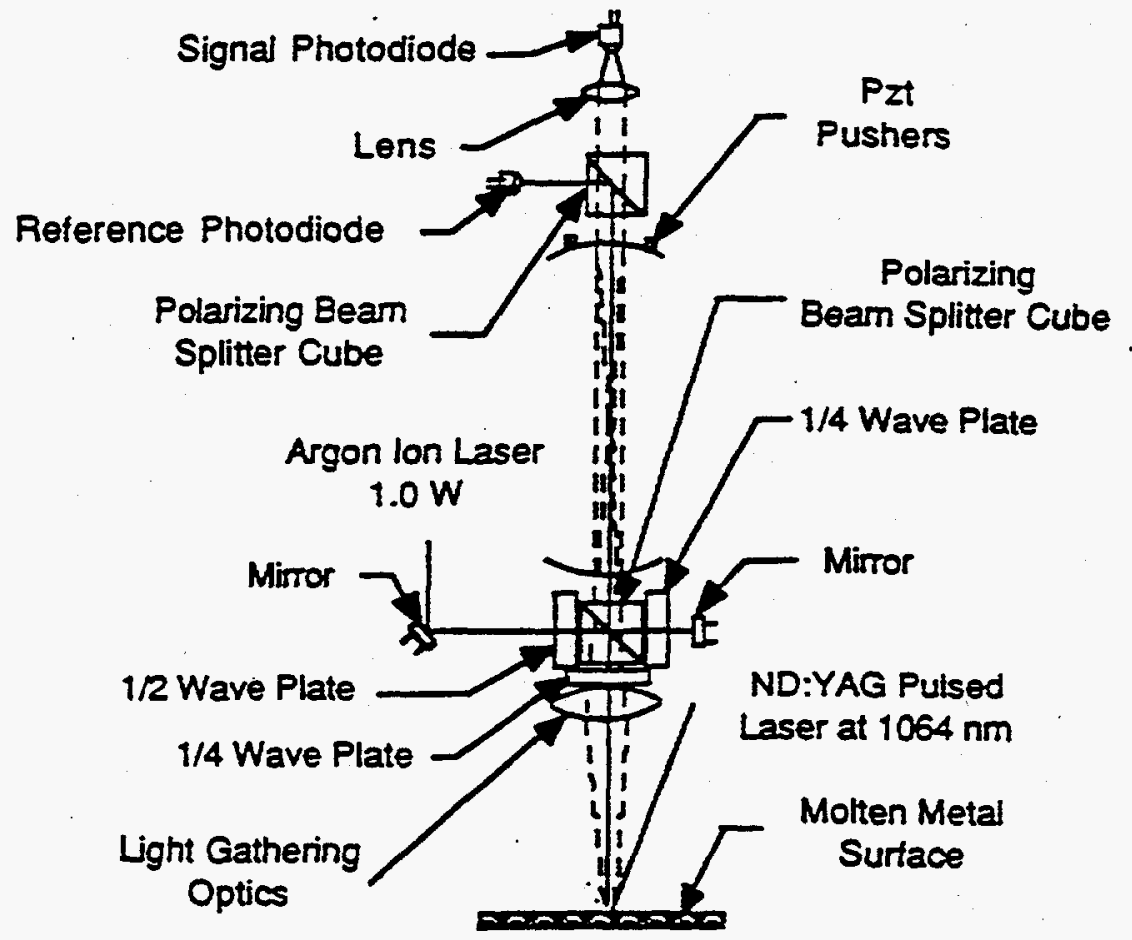

Figure 1. Laser ultrasonic measurement system. 
frequency/surface velocity. With this approach, responses can be collected from diffuse surfaces, polished surfaces are not required, and strict optical alignment is not necessary. The basic requirement for signal detection is that sufficient light is collected and passed though the interferometer to achieve an acceptable signal-to-noise ratio. The argon ion detection laser power ranged from 0.3 to $0.4 \mathrm{~W}$.

\section{Experimentation}

Experimentation proceeded in two phases. The first developed baseline data comparing conventional ultrasonics to laser ultrasonics by making measurements on solid samples. Due to the variability in surface conditions and geometry, only comparative measurements and results were obtained. The second, and most informative, phase was liquid metal measurements with laser ultrasound. The purpose of these measurements was to demonstrate feasibility and identify potential technical difficulties.

\section{Solid Samples}

Initial experimentation was performed on steel samples provided by members of The American Foundrymen's Society. Tables 1 and 2 list the samples provided and the processing variables used during their fabrication. The samples are rectangular plates approximately $255 \mathrm{~mm} \times 255 \mathrm{~mm}$ $x 15 \mathrm{~mm}$. Baseline measurements were made with conventional piezoelectric transducers on the as-received samples. A $20 \mathrm{MHz}$ immersion transducer was used to measure backscatter at five locations (A through $E$ ) on each sample, starting $25.4 \mathrm{~mm}$ from one edge and progressing inward (perpendicular to the edge) using $25.4 \mathrm{~mm}$ steps. Attenuation measurements were performed for each

plate at locations $A$ and $B$. The backscatter results for the five measurement locations are summarized in Figure 2. Comparative attenuation measurements for locations $\mathrm{A}$ and $\mathrm{B}$ are plotted in Figure 3. Significant measurement variations were observed for the different test locations within individual samples as well as from sample to sample. A compilation of the MATLABTM based computer program used to process the data, as well as the raw and processed data, is provided in Appendix A for the backscatter experiments and in Appendix B for the attenuation experiments.

In general, these results verify what is already known, that cast samples are microstructurally complex, and that phases, composition, grain size, porosity, and/or inclusions can vary within and between samples. These microstructural variables, individually and in combination, contribute to 
Table 1. Casting data on Alloy 1025 samples from Foundry 103."

\begin{tabular}{|c|c|c|c|c|c|c|}
\hline $\begin{array}{l}\text { Casting } \\
\text { Numberb }\end{array}$ & $\begin{array}{l}\text { Ladle } \\
\text { Sizec }\end{array}$ & Pouring Arrangement & Mold Type & $\begin{array}{c}\text { Pouring } \\
\text { Temperature }\left({ }^{\circ} \mathrm{F}\right)\end{array}$ & $\begin{array}{l}\text { Pouring } \\
\text { Time (s) }\end{array}$ & $\begin{array}{c}\text { Radiograph } \\
\text { Rating }\end{array}$ \\
\hline $103-602$ & $1500 \mathrm{lb}$ & Submerged pour, clay shroud & $\begin{array}{l}\text { Green sand, silica-zircon } \\
\text { splash basin }\end{array}$ & 2802 & 30 & 3 \\
\hline $103-605$ & $1700 \mathrm{lb}$ & AFS 1:2:2 Gating & Green sand & 2900 & 18 & 2 \\
\hline $103-606$ & $1700 \mathrm{lb}$ & AFS $1: 2: 2$ Gating & Green sand & 2900 & 18 & 2 \\
\hline $103-607$ & $1700 \mathrm{lb}$ & $\begin{array}{l}\text { AFS 1:2:2 Gating, mechanical } \\
\text { connection to ladle }\end{array}$ & Green sand & 2900 & 12 & 2 \\
\hline $103-608$ & $1700 \mathrm{lb}$ & $\begin{array}{l}\text { AFS } 1: 2: 2 \text { Gating, mechanical } \\
\text { connection to ladle }\end{array}$ & Green sand & 2900 & 12 & 2 \\
\hline $103-612$ & $1700 \mathrm{lb}$ & AFS $1: 2: 2$ Gating & Green sand & 2900 & 22 & 2 \\
\hline $103-616$ & $1500 \mathrm{lb}$ & Submerged pour, clay shroud & $\begin{array}{l}\text { Green sand, silica-zircon } \\
\text { splash basin }\end{array}$ & 2885 & 6 & 6 \\
\hline $103-618$ & -- & $\cdots$ & --- & --- & $\cdots$ & $\cdots$ \\
\hline $103-619$ & $\cdots$ & -- & --- & -- & $\cdots$ & $\cdots$ \\
\hline $103-620$ & -- & $\cdots$ & -- & $\cdots$ & $\cdots$ & $\cdots$ \\
\hline $\begin{array}{l}\text { a. Melted } \\
\text { b. Castin } \\
\text { c. Botton }\end{array}$ & $\begin{array}{l}\text { acid elect } \\
\text { umber is } \\
\text { ur. }\end{array}$ & $\begin{array}{l}\text { arc furnace with silica brick li } \\
\text { mped on edge of each plate. }\end{array}$ & Deoxidized with $\mathrm{Al}$ sh & Graphidox). & & \\
\hline
\end{tabular}


Table 2. Casting data on carbon steel samples from Foundry $111 .{ }^{a}$

\begin{tabular}{|c|c|c|c|c|}
\hline $\begin{array}{l}\text { Casting } \\
\text { Number }\end{array}$ & Ladle Treatment & $\begin{array}{c}\text { Pouring } \\
\text { Temperature }\left({ }^{\circ} \mathrm{F}\right)\end{array}$ & $\begin{array}{l}\text { Pouring } \\
\text { Time (s) }\end{array}$ & $\begin{array}{l}\text { Radiograph } \\
\text { Rating }\end{array}$ \\
\hline $111-101$ & Ca wire inject only; $0.73 \mathrm{lbs} /$ ton; $37 \mathrm{ppm}$ recovery & -- & -- & 7 \\
\hline $111-104$ & $\begin{array}{l}\text { Ca wire + argon; stir } 0.57 \mathrm{lbs} \mathrm{Ca/ton;} 35 \mathrm{ppm} \text { recovery; stir } 1 \mathrm{~min} \text {. } \\
\text { at } 30 \mathrm{CFH} \text { prior to } \mathrm{Ca}, 1 \mathrm{~min} \text {.; at } 20 \mathrm{CFH} \text { after } \mathrm{Ca}\end{array}$ & -- & - & 5 \\
\hline $111-105$ & Argon stir only; $8 \mathrm{~min}$ at $20-25 \mathrm{CFH}$ & - & -- & 6 \\
\hline $\begin{array}{l}\text { a. Melted } \\
\text { calcium alt } \\
\text { b. Casting }\end{array}$ & $\begin{array}{l}\text { basic electric arc furnace. } 15 \text { ton bottom pour ladle, } 70 \% \text { alumina line } \\
\text { inate ladle addition. } \\
\text { lumber is stamped on edge of each plate. }\end{array}$ & Deoxidized with & nge (Fe & $15 \mathrm{lb} / \mathrm{ton}$ \\
\hline
\end{tabular}






Sample Number

Sample

103-602

103-605

103-606

103-607

103-608

103-612

103-616

103-618

103-619

103-620

111-101

111-104

111-105

Figure 2. Ultrasonic backscatter data for cast steel samples.

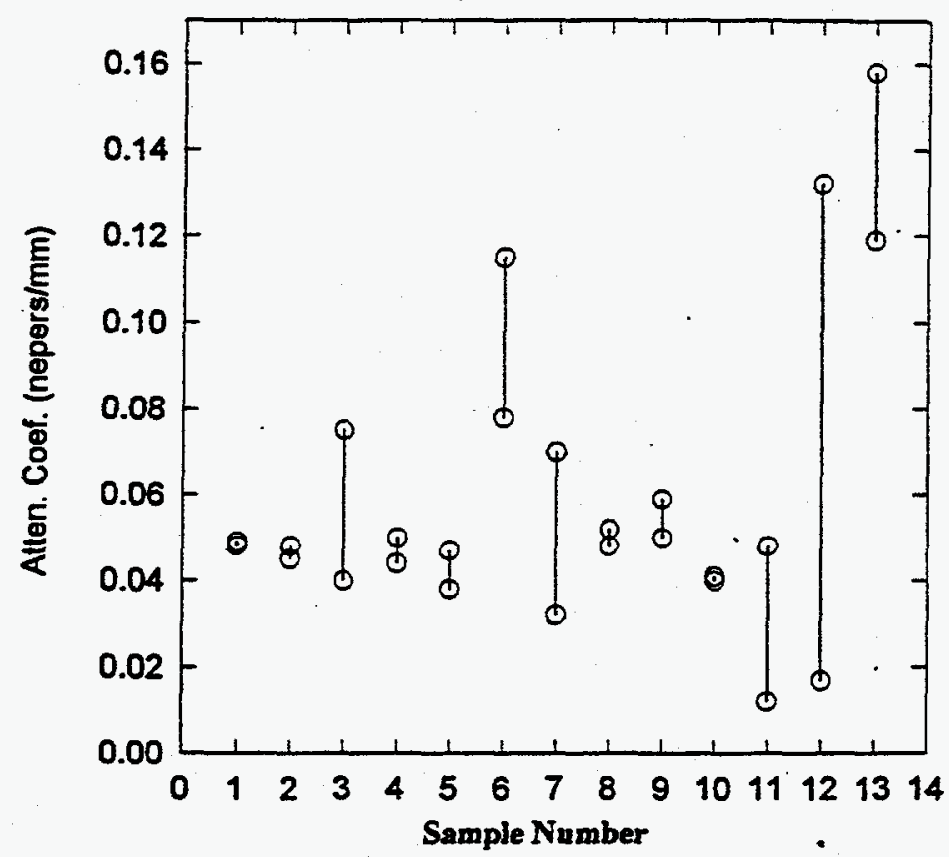

Sumple Number

Sample

103-602

103-605

$103-606$

103-607

103-608

103-612

103-616

103-618

103-619

103-620

111-101

111-104

111-105

Figure 3. Ultrasonic attenuation data for cast steel samples. 
backscatter and attenuation and their effects cannot be separated without additional information. Because of the complexity of the samples, the results obtained are not useful for addressing the objective of this task. However, the results do point out the potential of UT to monitor the variability of cast samples. Of the two measurement techniques used for these samples, attenuation will be less reliable than backscatter because of its sensitivity to surface conditions and interface geometries. A limited examination of the backscatter data indicates that the average backscatter values of samples 103-602, 103-606, 111-101, and 111-105 are higher than those of the other samples; this may be indicative of increased porosity or inclusions (assuming that the microstructure is similar for all samples). The highest backscatter values for samples 103-606 and 111-105 can be explained by coarse porosity revealed by the radiographs at the corresponding test locations. Interpretation and correlation of the attenuation results with the backscatter results is impractical because of unknown variations in microstructure, composition, and geometry.

Laboratory-fabricated tin samples were used to address sample complexity. Samples were prepared by swaging tin powder $(\leq 45 \mu \mathrm{m}$ ) seeded with different quantities of iron powder (180 to $250 \mu \mathrm{m})$ in a die. The resulting fine-grain solid samples were $97 \%$ dense with an iron distribution adequate for experimentation. Ultrasonic scatter measurements were performed using both piezoelectric and laser transducers. Figure 4 is a summary of the piezoelectric data. As can be seen, the scatter measured is proportional to the iron content. Figure 5 is a summary of the laser ultrasonic measurements. The results follow the same trend as the piezoelectric measurements, but the lower signal-to-noise ratio associated with the existing laser system resulted in decreased precision. Figure 6 contains three laser ultrasonic A-scans illustrating the increase in scatter signal with increased iron content. Although the A-scans cannot be directly compared because of sample thickness variations, they illustrate the effects of ultrasonic attenuation by the dramatic decrease in amplitude of the second pressure wave compared to the first. The second pressure wave is the remains of the first pressure wave after it has made a round trip

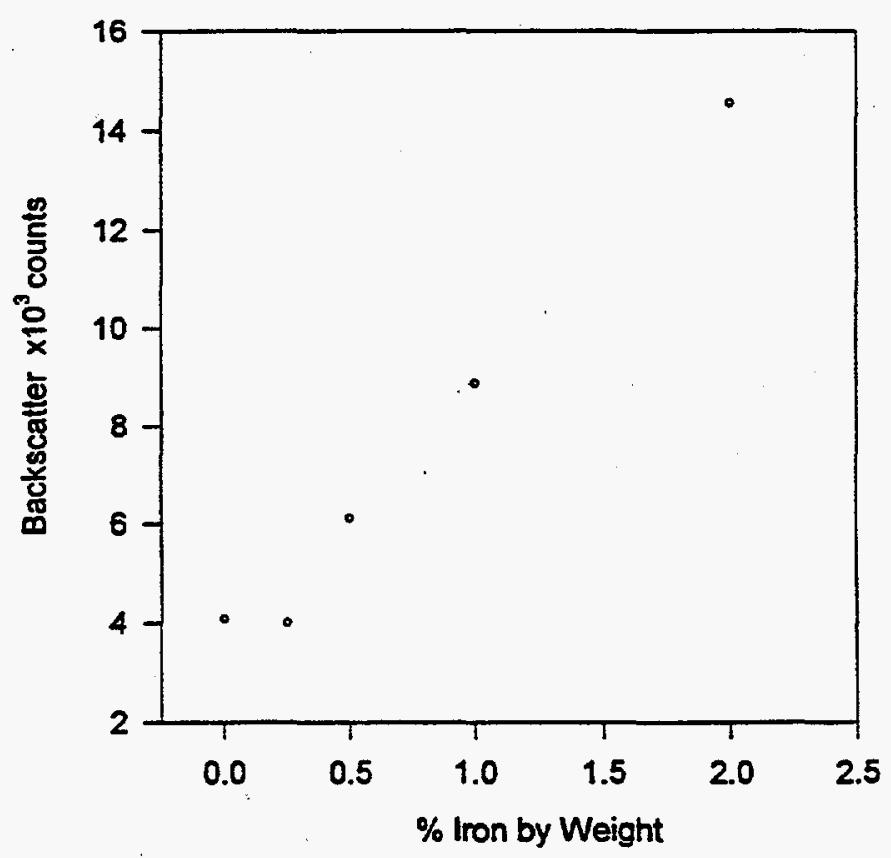

Figure 4. Ultrasonic scatter data for $\mathrm{Sn}-\mathrm{Fe}$ samples obtained with piezoelectric-based system. 


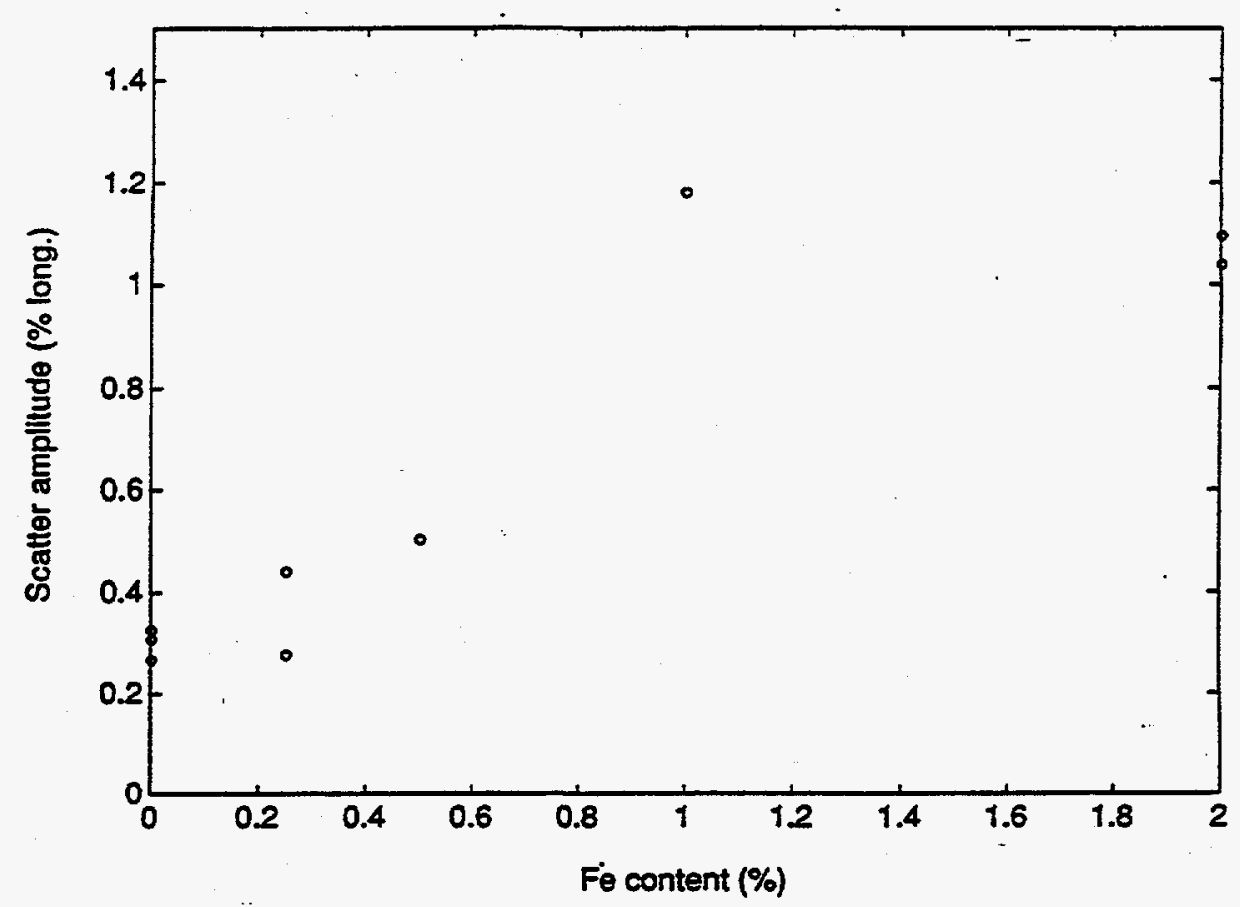

Figure 5. Ultrasonic scatter data for $\mathrm{Sn}-\mathrm{Fe}$ samples obtained with laserbased system.

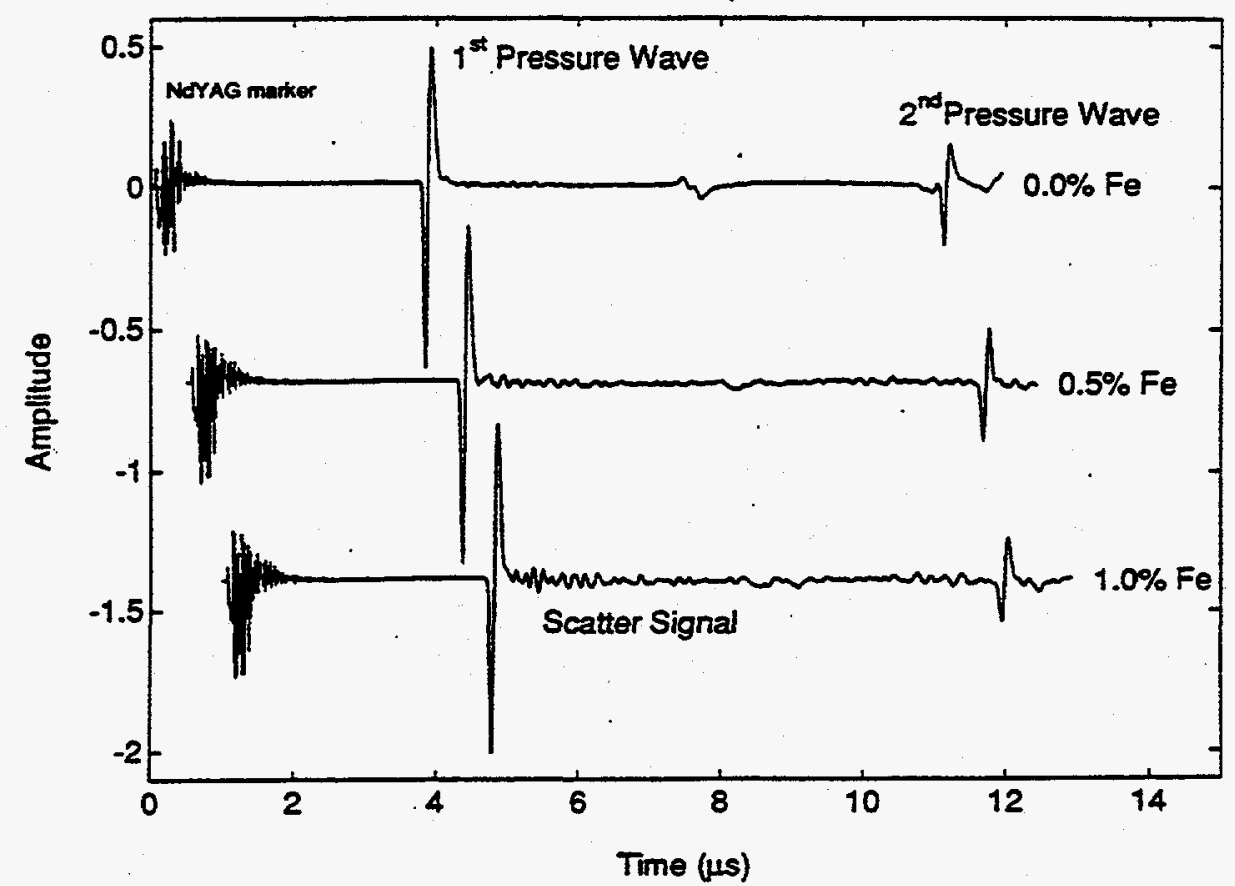

Figure 6. Laser ultrasonic data illustrating increased scatter with increased Fe content. 
through the sample via reflection at the front and back surfaces. These results demonstrate the feasibility of laser ultrasonics for the type of measurements deemed most useful for measuring inclusions in liquid metals.

\section{Liquid Metal}

Experiments were performed using liquid tin seeded with iron powder (250 to $500 \mu \mathrm{m}$ particle size). The intent of these experiments was to examine a number of tin melts containing different inclusion concentrations. However, a problem was encountered in maintaining a known distribution of iron powder in the melt. After initial agitation to homogenize the melt, the iron quickly settled to the bottom, resulting in unknown distributions and/or concentrations. Because of the time required to allow surface turbulence to subside, no meaningful measurements could be obtained when using the free surface of the melt to produce and detect ultrasound. Side wall, through transmission measurements proved to be more successful. Both backscatter and attenuation measurements were performed.

The backscatter measurements were deemed unsuccessful because of the inability to reliably detect the inclusions with the low signal-to-noise ratio encountered. With this laser ultrasound system and application, the largest focal spot practical for generating ultrasound is approximately $6 \mathrm{~mm}$. This results in considerable diffraction of the beam. A higher power laser would allow the generation focal spot to be increased, thereby decreasing the diffraction of the beam. This would improve the signalto-noise ratio by increasing the energy density of the propagating wave. It is presently unknown if sufficient increase in the signal-to-noise ratio could be achieved to detect the backscatter of the inclusions.

Figure 7 shows two sets of attenuation measurements, illustrating the ability to monitor melt inclusion content over time. The acoustic attenuation of the melt decreases with time because the iron powder is settling to the bottom, leaving fewer suspended particles to scatter the propagating beam. These results demonstrate the feasibility of laser ultrasonics to detect and monitor inclusions in liquid metal.

\section{Technical Issues}

When using the free surface of the liquid metal for the generation and detection of ultrasound, surface motion due to turbulence, external vibration, and/or momentum imparted by the pulsed 


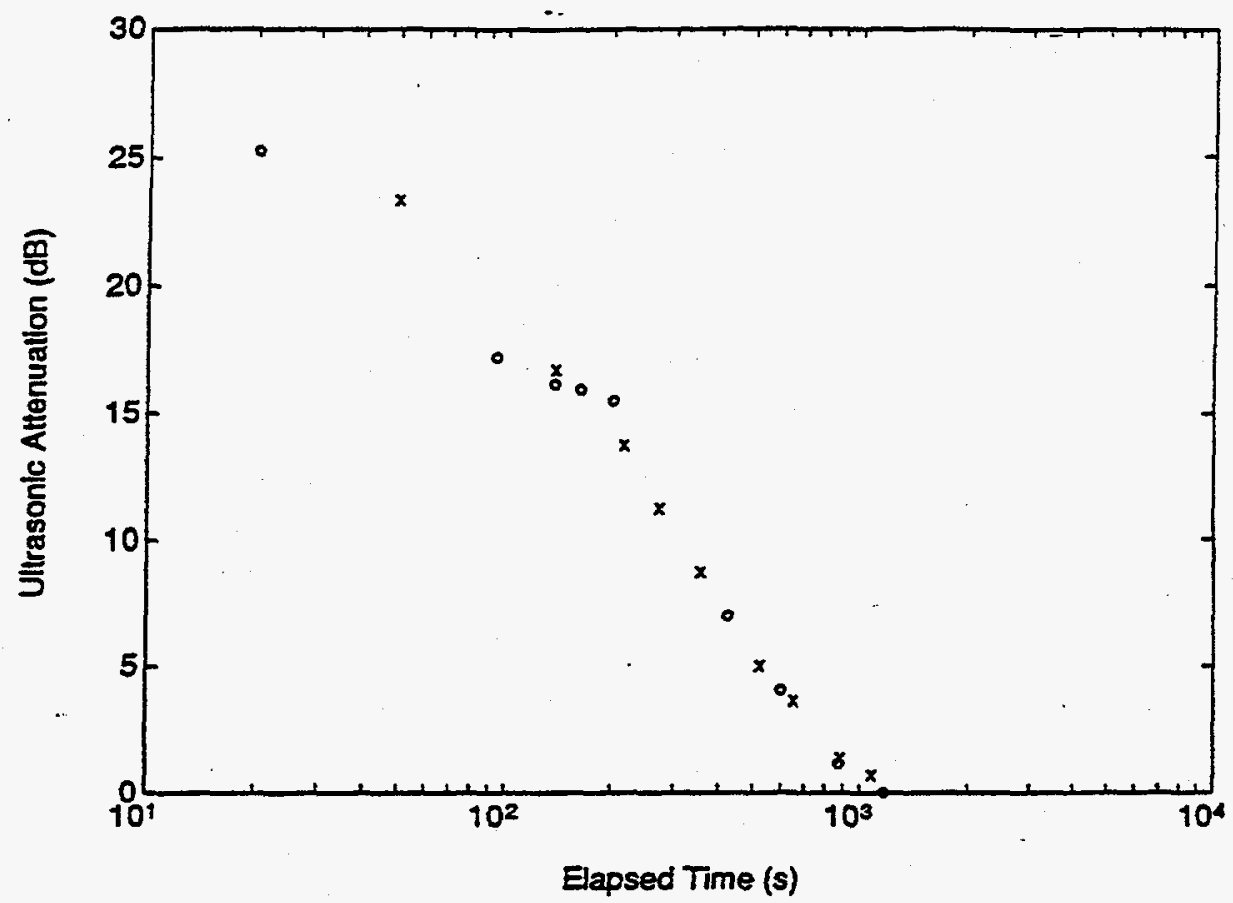

Figure 7. Laser ultrasonic attenuation measurements of liquid tin containing Fe powder.

generation laser can scatter parts or all of the detection laser beam beyond the acceptance angle of the optics. This results in inconsistent signal detection. Similarly, variations in surface reflectivity due to oxidation reduce the amount of laser light available for detection, thereby reducing the signal-to-noise ratio. Another undesirable phenomenon is the production of an atmospheric shock wave by the generation laser as it briefly disrupts the detection laser. Any ultrasonic signal coinciding with the disruption will be lost. It is believed that these problems will not prevent the use of laser ultrasonics for the detection of inclusions in liquids, but they present some limitations on how the technology is applied and the information acquired. Finally, the limited sensitivity of the laser-based detectors will most likely prevent practical ultrasonic bulk backscatter measurements. This limits the technique to attenuation measurements, which require known path lengths and controlled interfaces that are inserted into the process. stream and thus loses the advantage of being totally noncontacting.

\section{Conclusions and Recommendations}

Laser ultrasonic attenuation measurements can be used to detect and monitor inclusions in liquid metals. However, the limits of detectability (size and concentration) have not been established and implementation of the technique will require specific conditions whose compatibility with industrial processes are presently unknown. The limitations identified may reduce laser ultrasonics to a 
contacting form of measurement that is nearly equivalent to piezoelectric measurements and thus technically too complex and cost ineffective.

To determine the feasibility of industrial implementation, as well as any advantages over piezoelectric methods, it is recommended that additional development work be performed to address the stated caveats. This development effort will require industrial support and direction in the form of:

1) Consultation on the practicality, mechanisms, and economics of integrating laser ultrasonic attenuation measurements with industrial processes.

2) Stock material having known inclusion concentrations and size distributions representative of industrial processes. It will be necessary to initially use a low melting point material such as aluminum to simplify laboratory-based experimentation. Ferrous materials can be addressed when the laser technology is deemed to be useful and implementable.

3) Direction on proper melt practices for the stock material will to prevent alteration of the known properties.

\section{References}

1. D. I. Walker et al., "The Development of Ultrasonic Sensors for the Early Detection of Slag Carryover," Proc. 9th Process Technology Conference, March 25-28, 1990, Detroit, MI.

2. N. D. G. Mountford et al., "Progress in the Development of an Ultrasonic Sensor for the Measurement of Liquid Metal Cleanliness," Proc. Steelmaking Conference, Iron and Steel Soc., A.I.M.E., pp. 773-781, 1991.

3. N. D. G. Mountford et al., "The Relationship Between Inclusion Contents as Measured by Ultrasonic Testing in Liquid Steel and the Cast Metal Quality," Proc. Electric Furnace Conference, pp. 243-248, 1991.

4. R. I. L. Guthrie and H. C. Lee, "On-Line Measurements of Inclusions in Steelmaking Operations," Proc. Steelmaking Conference, Iron and Steel Soc., A.I.M.E., pp. 799-805, 1992.

5. S. Dawson, "The Application of Ultrasonics to the Evaluation of Liquid Steel Cleanliness," M. A. Sc. Thesis, University of Toronto, 1987.

6. T. L. Mansfield, "Ultrasonic Technology for Measuring Molten Aluminum Quality," Materials Evaluation, Vol. 41, No. 5, pp. 743-747, 1983. 
7. N. D. G. Mountford, "Part III: The Detection of Impurities in Liquid Aluminum," British Aluminum Company Limited, Report No. 50/6, March 29, 1950.

8. G. V. Garcia et al., "Noncontacting Laser Ultrasonic Generation and Detection at the Surface of Molten Metal," Review of Progress in Quantitative Nondestructive Evaluation, ed. D. O. Thompson and D. E. Chimenti, Plenum Press, New York, Vol. 9B, 1990, pp. 1981-1986.

9. V. W. Kurz and B. Lese, Berg-und-Huttenmabblsche Monatshefte, Vol. 114, pp.123-130, 1969. 


\section{Appendix A \\ Ultrasonic Backscatter Data For Cast Iron Samples}

Appendix A contains a compilation of the ultrasonic backscatter data for the cast steel samples supplied by the American Foundrymen's Society. Provided is a listing of the MATLAB ${ }^{\mathrm{TM}}$ computer program used to process the data followed sequentially by the raw ultrasonic A-scans and measured backscatter values for each sample. 
\%scatter.m

\%compares the scatter or noise for an ascan from a ROI defined by the

\%user, usually in between the front and back reflections.

\%T. K. O'Brien jan 14, 1994

$t f=1 e-6 ; t b=3.5 e-6 ;$ freq $=100 e 6 ;$ field $=301: 305 ;$ cont $=1 ; \%$ initial param eters

while cont $==1$;

fr=input (['enter sampling frequency [', num2str(freq/le6),' Mhz]: ' ])*1e6;

if $\mathrm{fr}^{-}=[\mathrm{]}$; frequfr; end

tl=input (['time past front scatter $[$ ', num2str(tf/le-6),' us]: '])* $1 e-6$;

if $t 1^{-}=[] ; t f=t l$; end

t2=input (['time for ROI [', num $2 \operatorname{str}(t b / 1 e-6), '$ uS ]: '])*1e-6;

if $t 2^{-}=[] ; t b=t 2$; end

fie=input (['enter data file range (current range [', num2str(field( 1)), ': ', num2str(field (length (field))), ']): ']);

if fie $^{-}=[]$; field=fie;end

sample=('sample name? '):

$t=[1: 1024]^{\prime} . * 1 /$ freq;

for $i=f i e l d ;$

file $=[$ 'wd', num2str(i), '.txt'] :

eval (['load ',file]); wd=eval (['wd', num2str(i)]);

off=mean (wd):

$w d=w d-m e a n(w d)$;

$k=f$ ind $(a b s(w d)>100) ; n=k(1)+t f * f r e q: k(1)+(t f+t b) * f r e q ; n 1=n(1) ; n 2=n($ length $(n))$;

energy=sum $(\operatorname{abs}(w d(n)))$;

esample $(i)=e n e r g y$;

$t 1=$ ones $(1,51) * t(n 1) ; t 2=$ ones $(1,51) * t(n 2) ; h=-300: 12: 300$;

figure (1), plot (t, wd, t(n), wd (n), tl,h, '. ',t2,h, '. ');

title(['File: ',file(1:length(file)-4),' Energy density: ', num2s tr(energy),' offset: ', num2str(off)]);

xlabel ('time (sec)'):

ylabel ('amplitude (cnts)');

text (1000,-280, ['offset: ', num2str(off)]);

text (1000,-230, ['Energy density: ', num2str(energy)]);

\%pause 
print

end

figure (2),plot (field, esample (field), field, esample (field), '0'), xlabel ('file numbers')

ylabel ('backscatter (total cnts)')

set (gca, 'xtick', field)

print

pause

\%text (axis(1)+10*axis (1), axis (2)+10*axis(2), 'Edge of Sample');

field=field+5;

cont=input ('continue? (1/2) ')

end 
File: wd301 Energy density: $1.292 e+004$ offset: 0.0004453

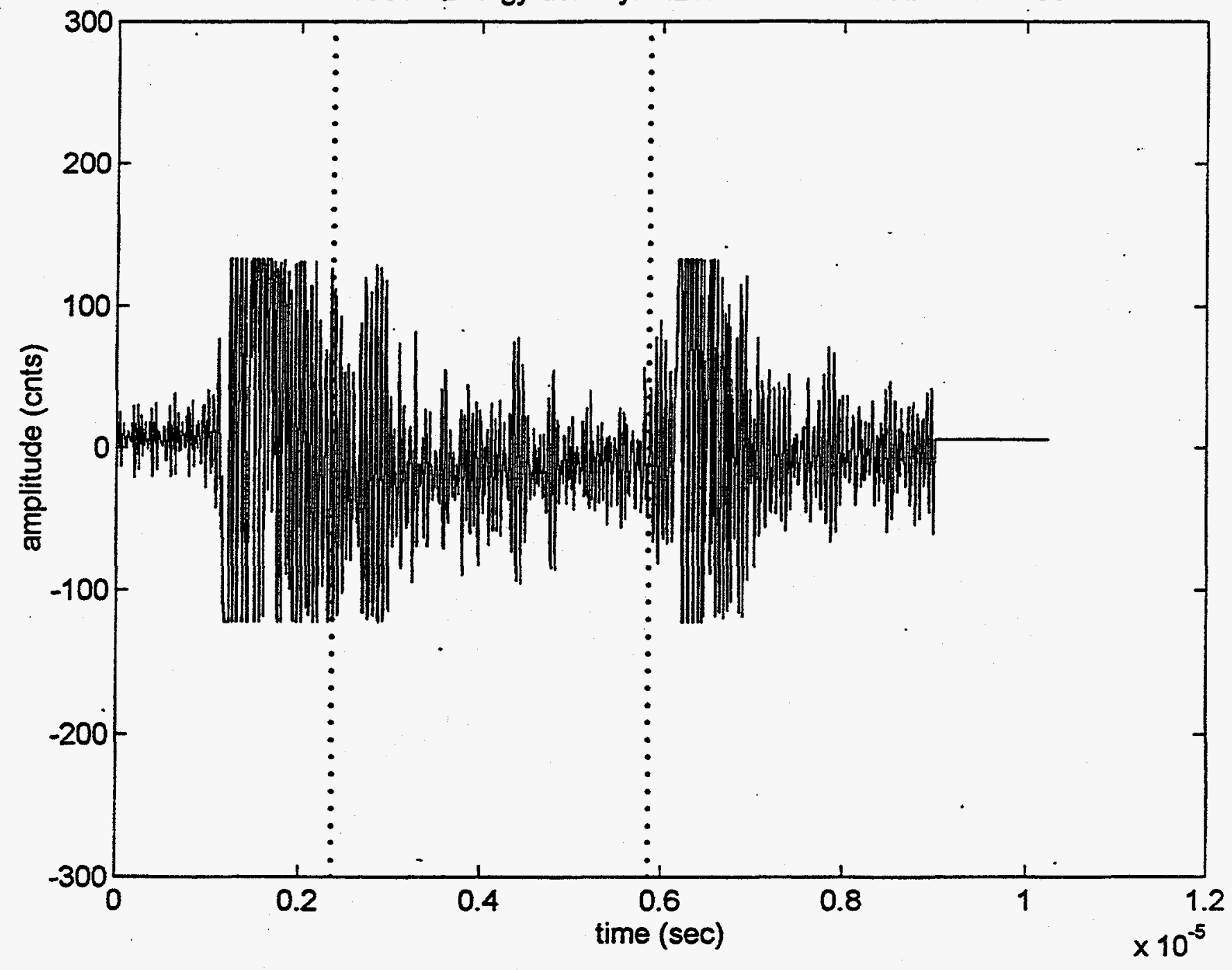


File: wd302 Energy density: $1.225 e+004$ offset: -0.000375

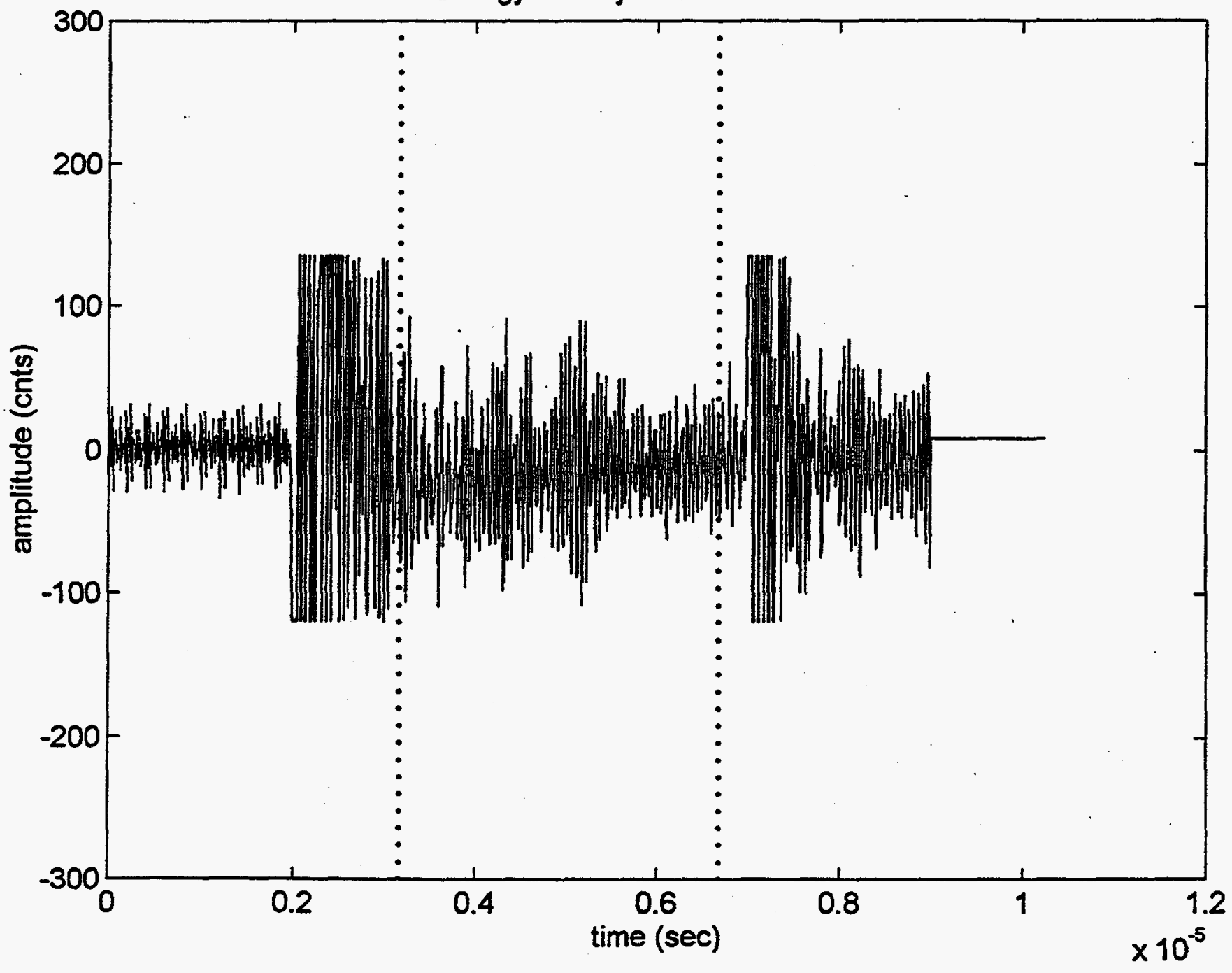


File: wd303 Energy density: $1.202 e+004$ offset: 0.0002812

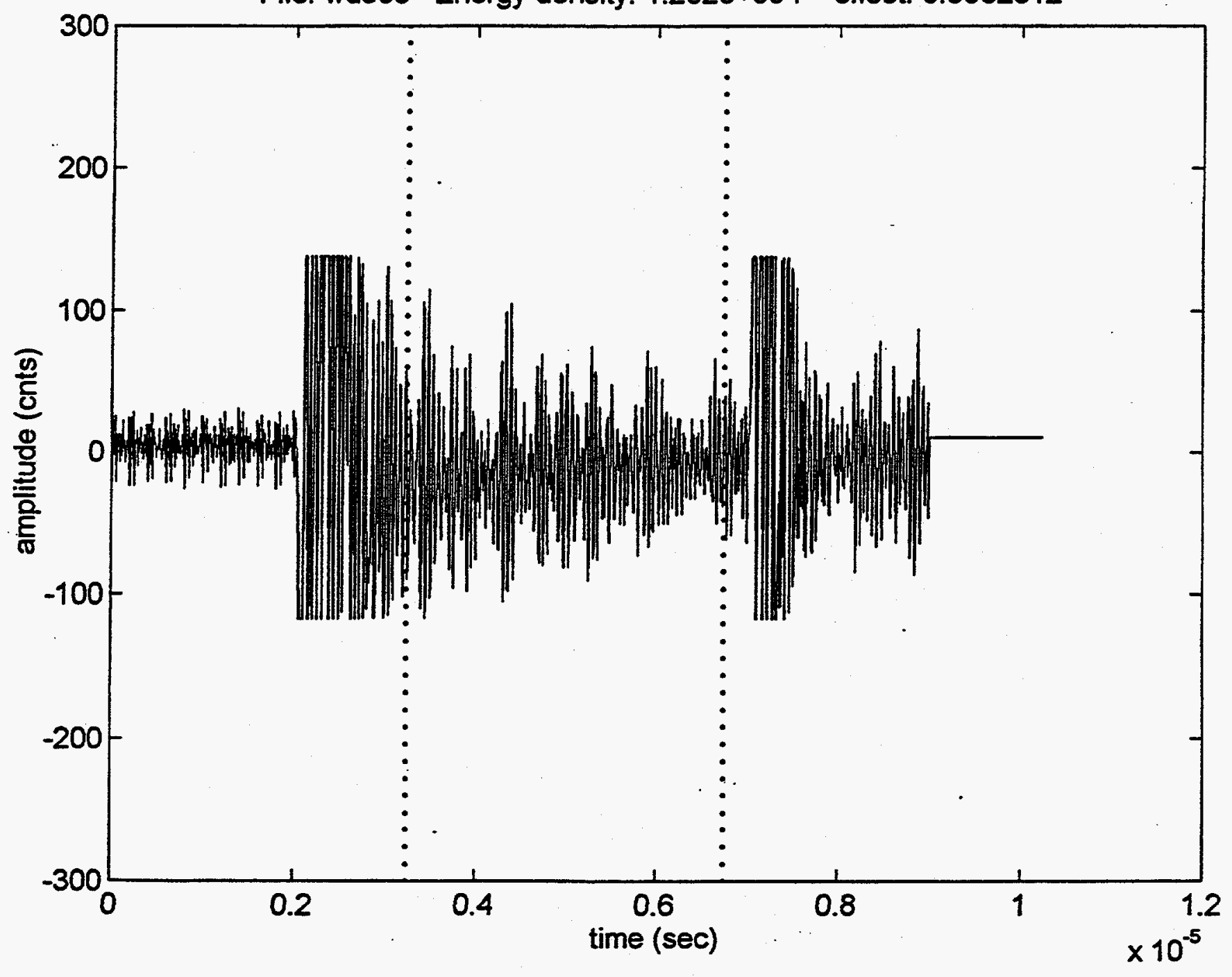







File: wd305 Energy density: 1.676e+004 offset: -0.0001563

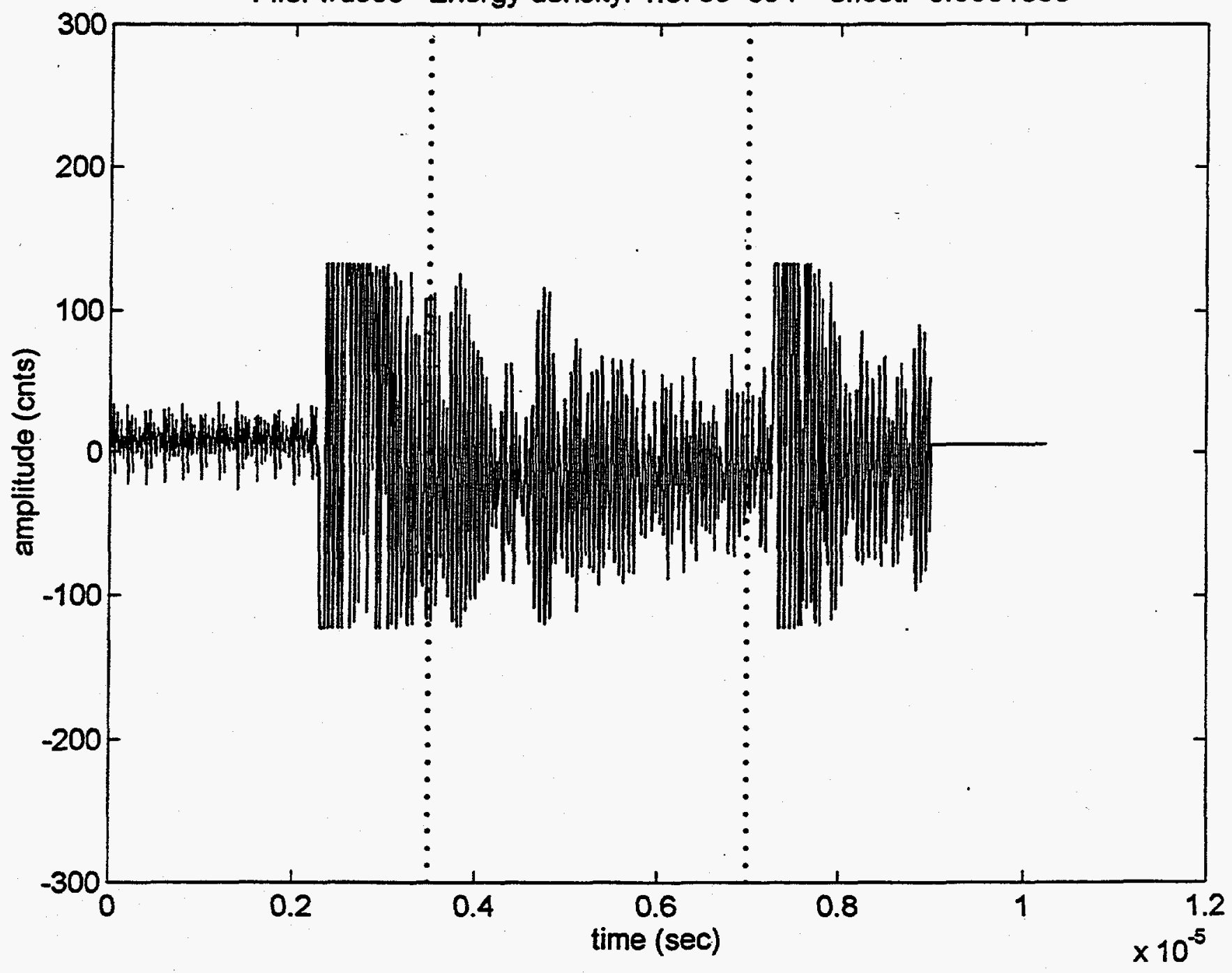




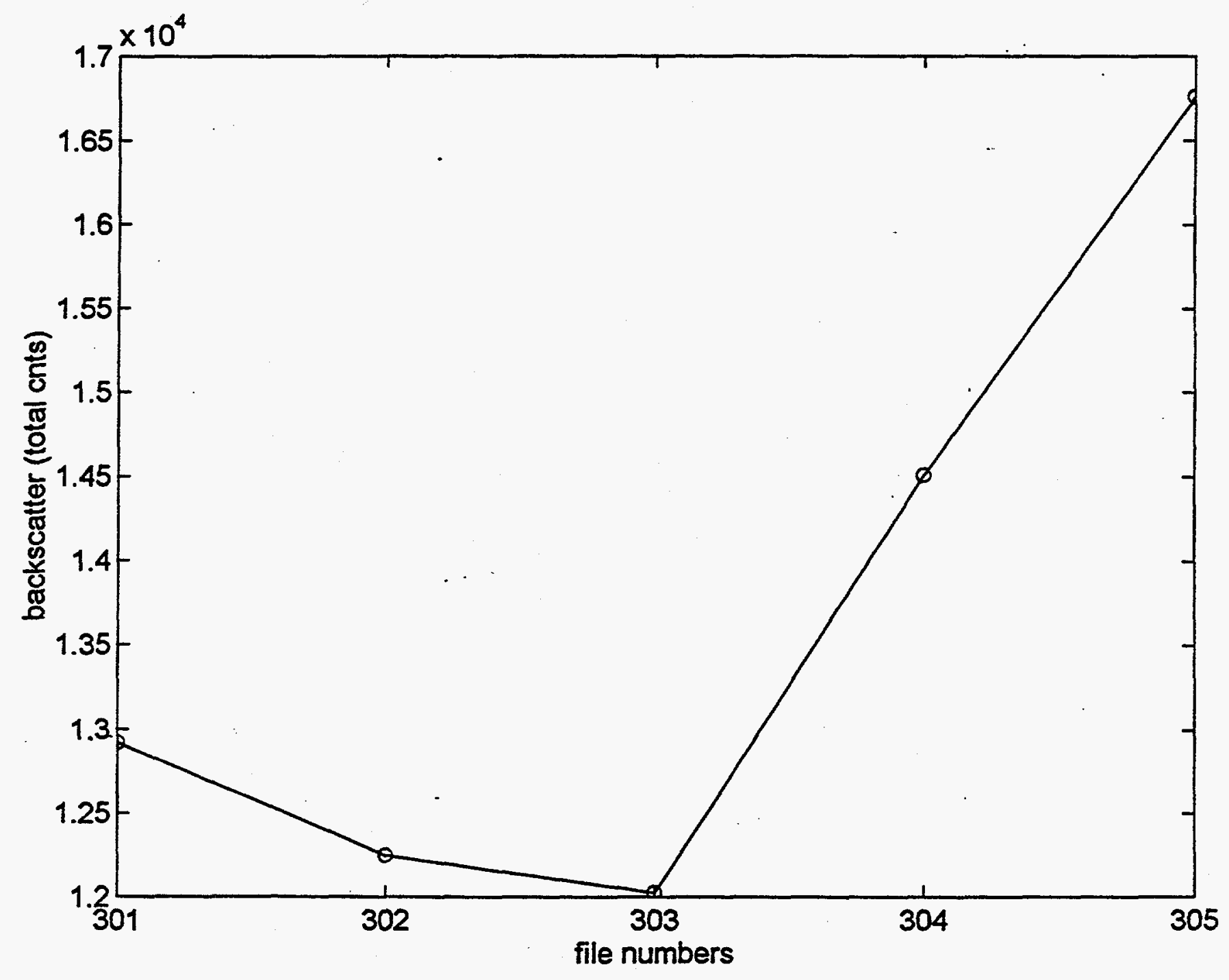


File: wd306 Energy density: 1.18e+004 offset: $-7.812 e-006$

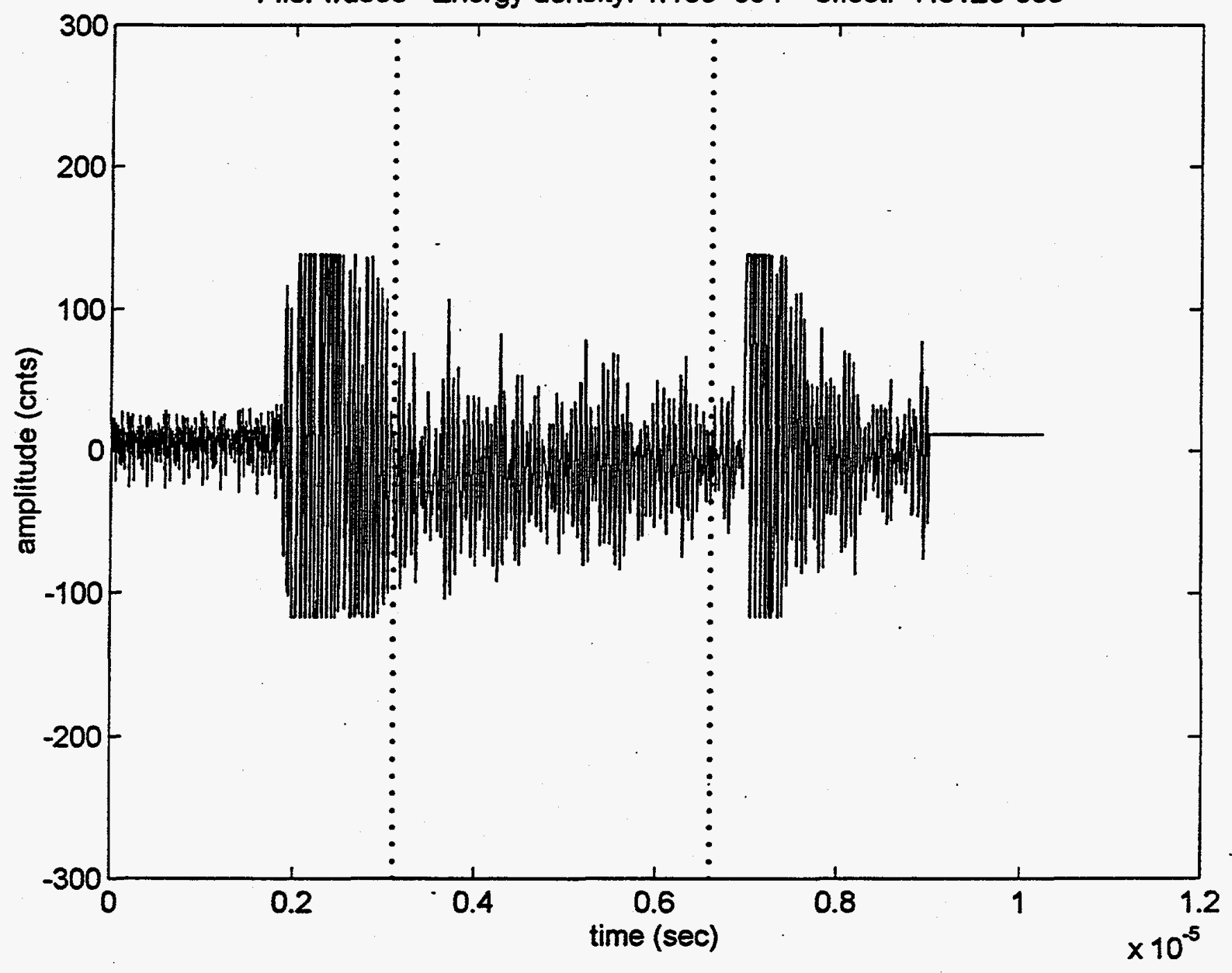


File: wd307 Energy density: $1.216 e+004$ offset: -0.00007031

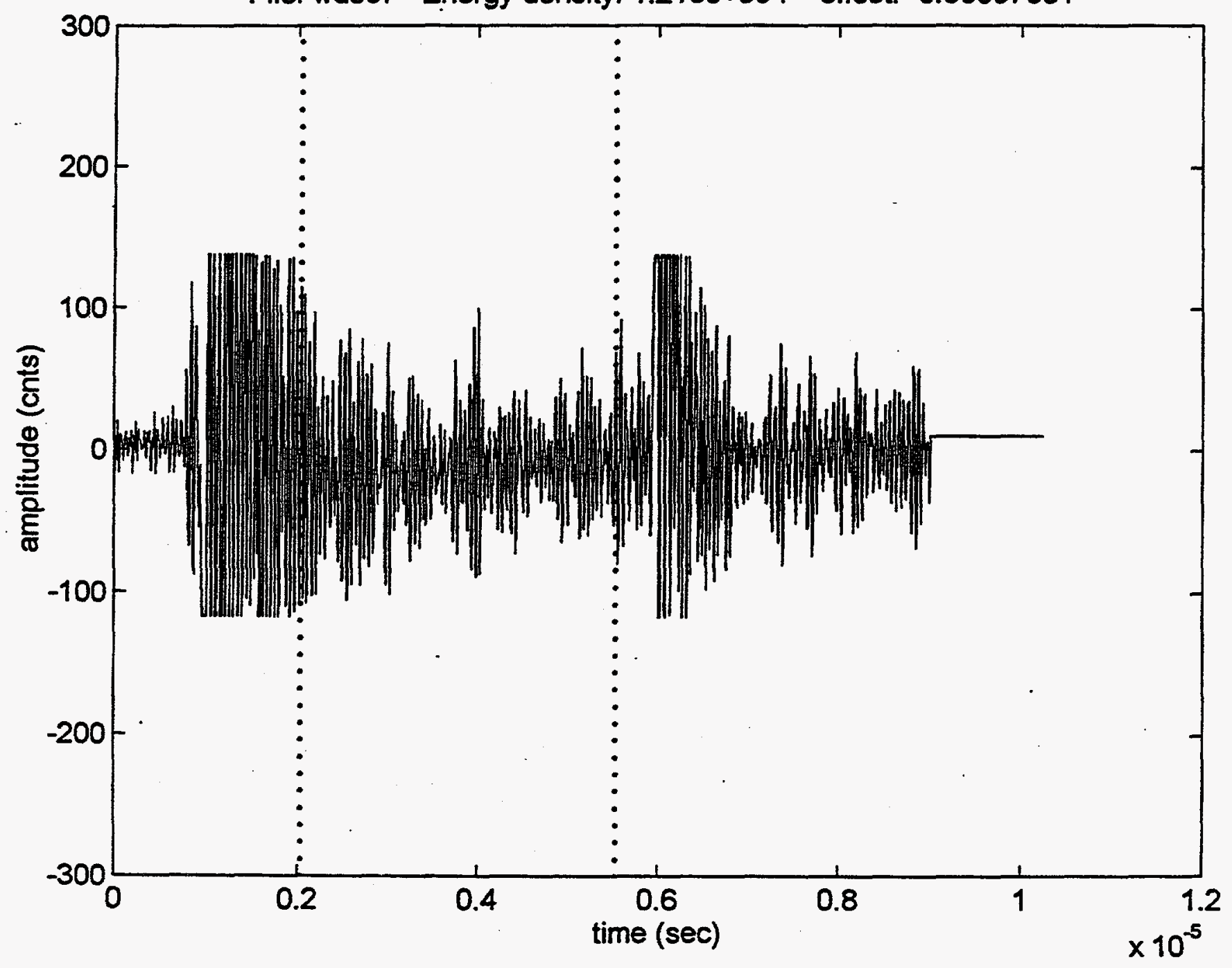


File: wd308 Energy density: $1.295 e+004$ offset: -0.00003125

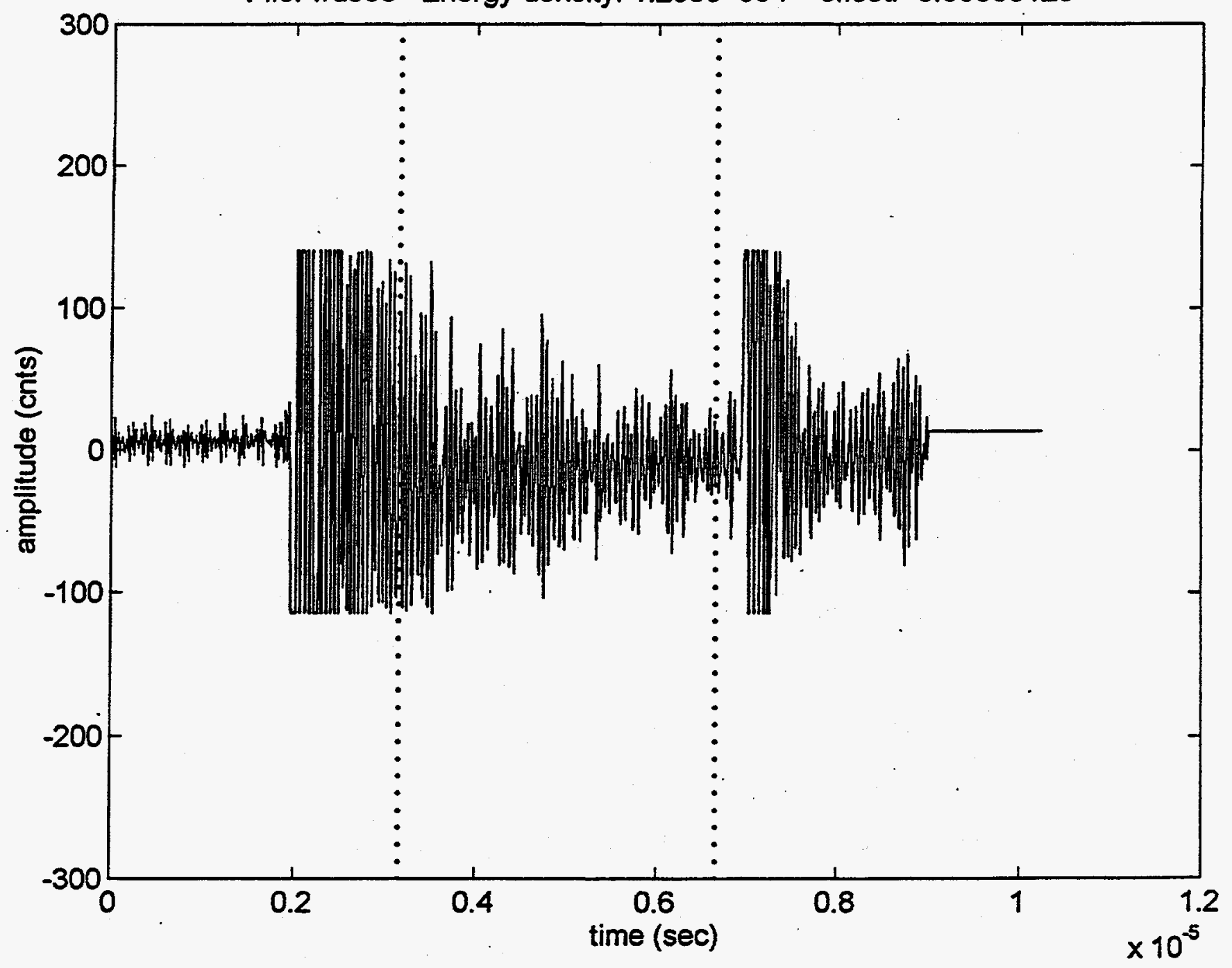


File: wd309 Energy density: $1.414 e+004$ offset: -0.0001875

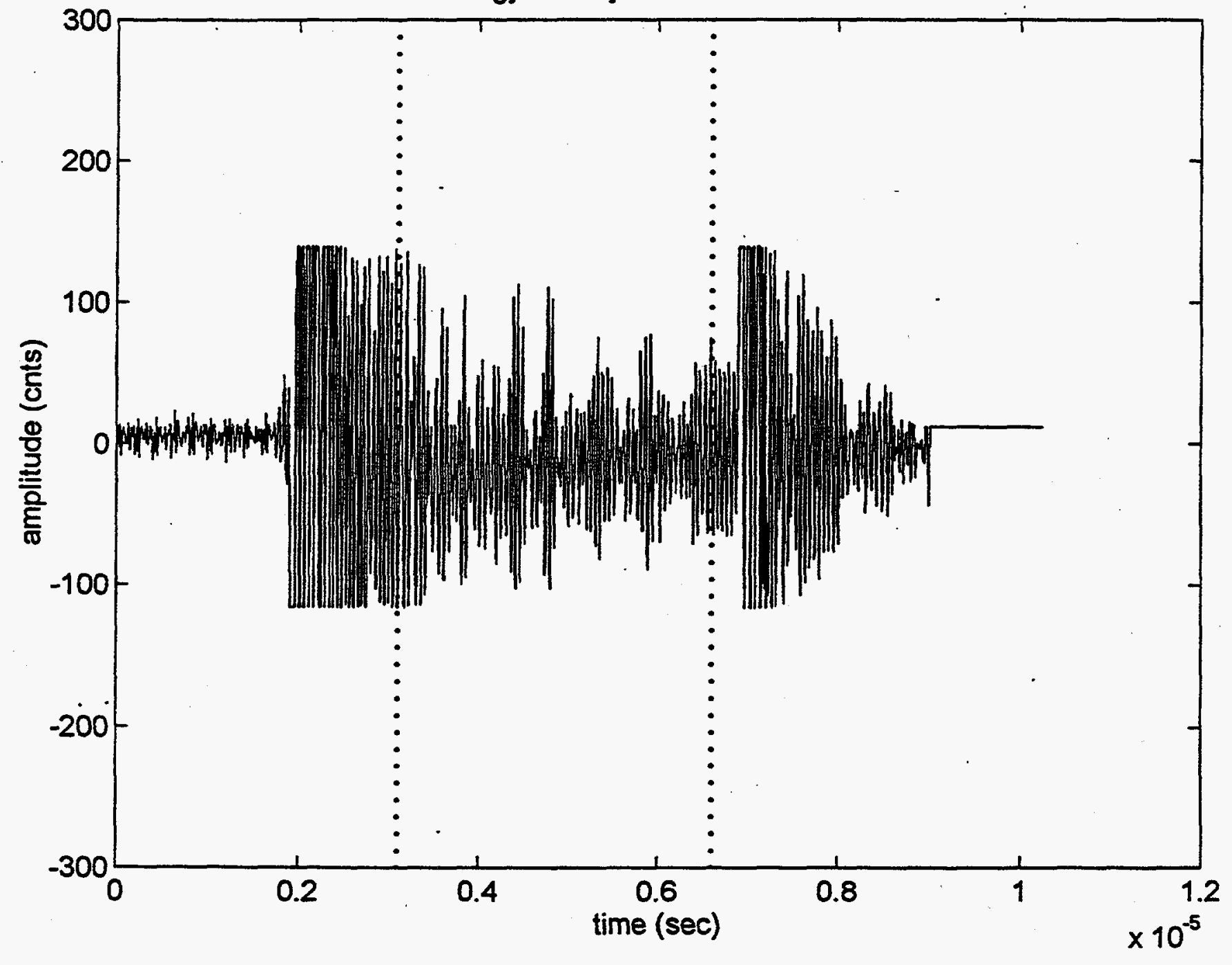


File: wd310 Energy density: $1.509 e+004$ offset: 0.0003359

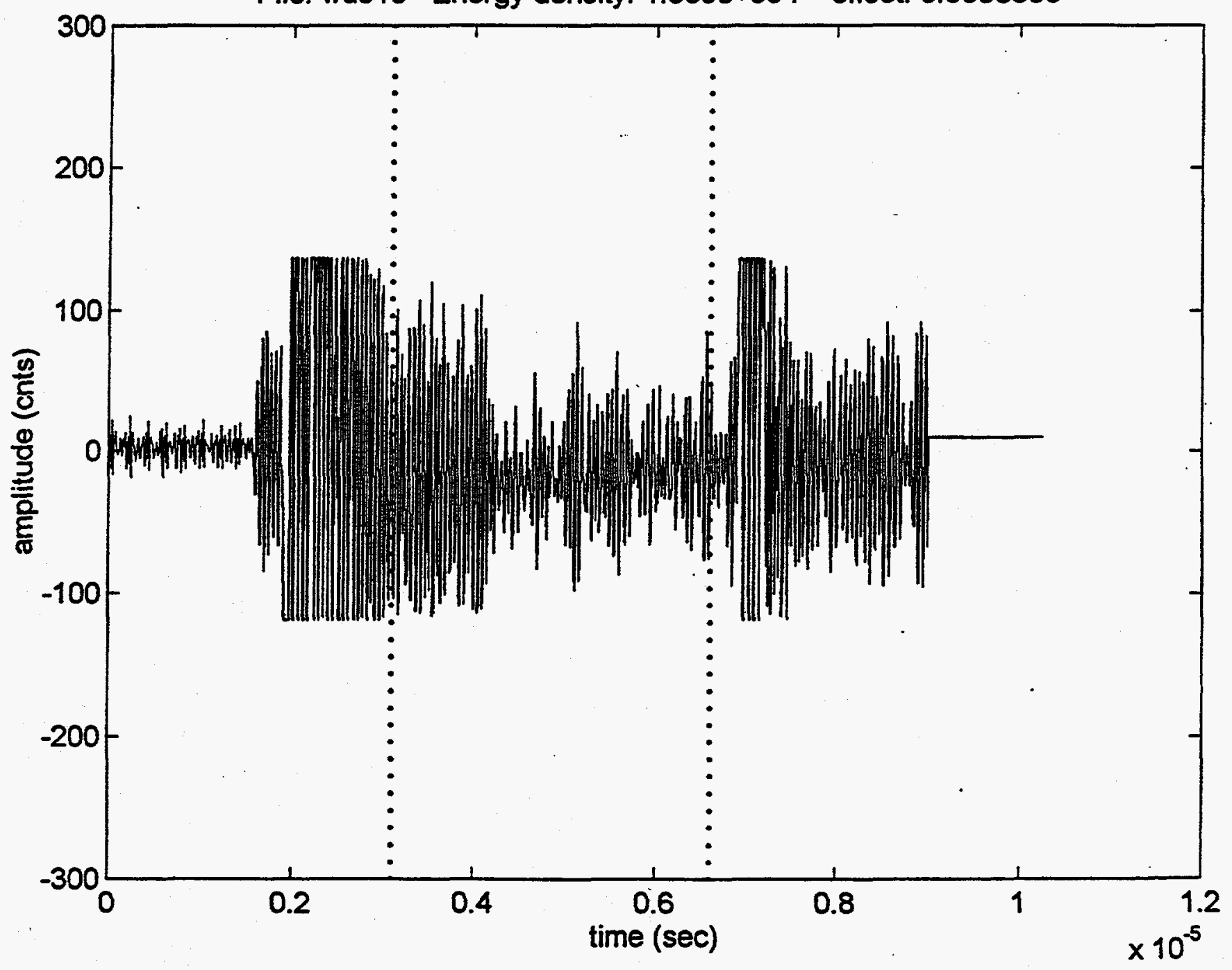




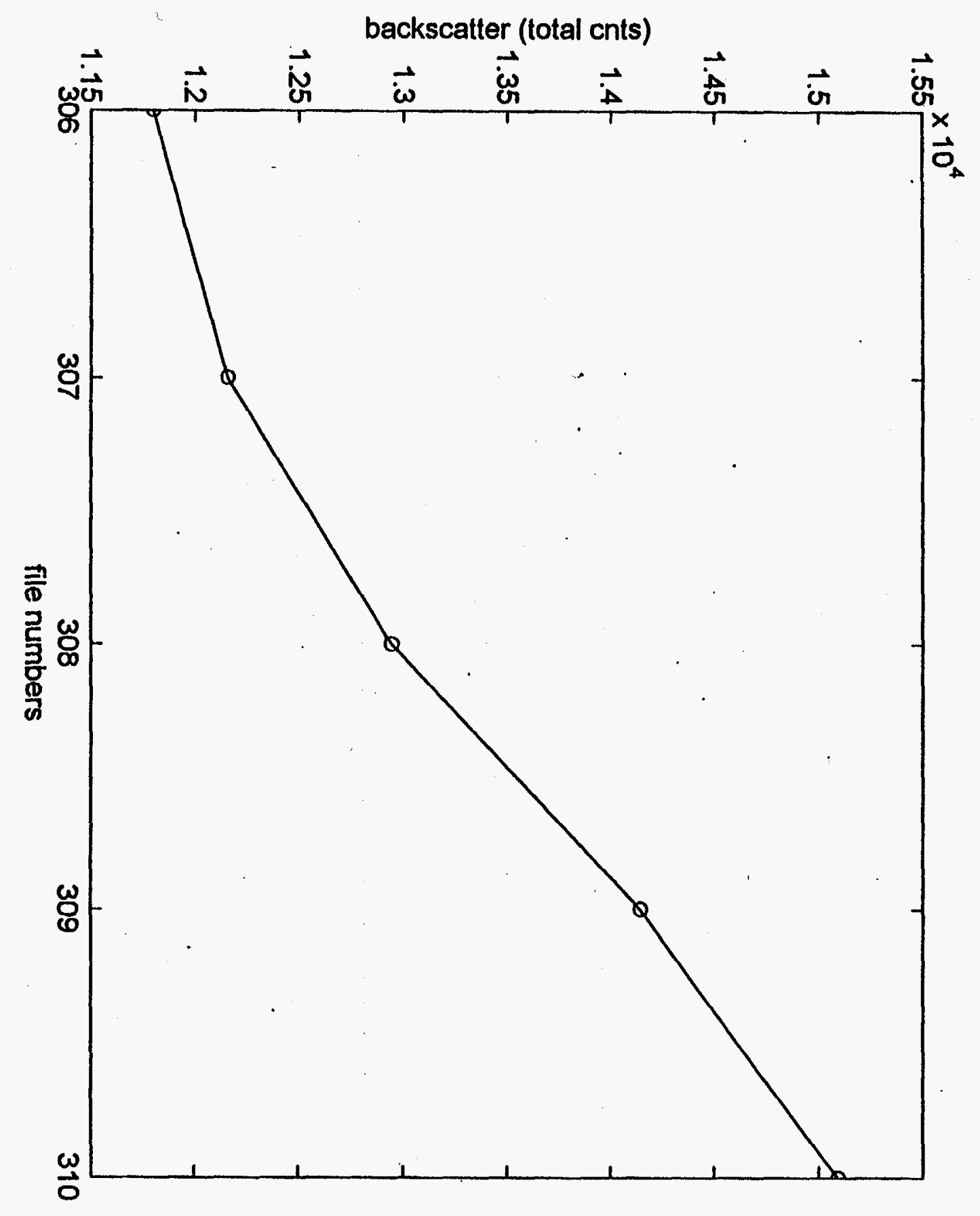


File: wd311 Energy density: $2.034 e+004$ offset: $-9.766 e-007$

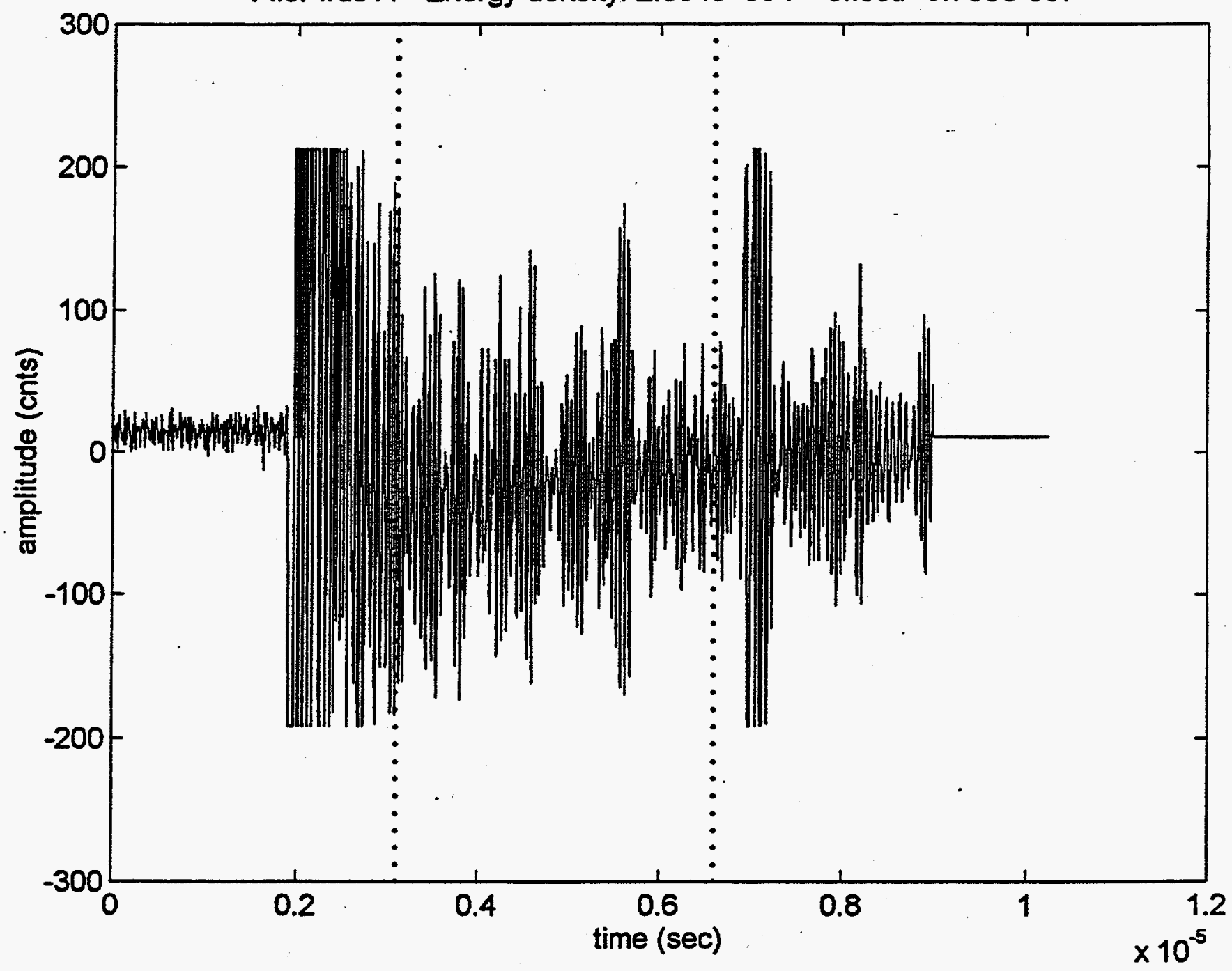


File: wd312 Energy density: $1.842 e+004$ offset: -0.0003672

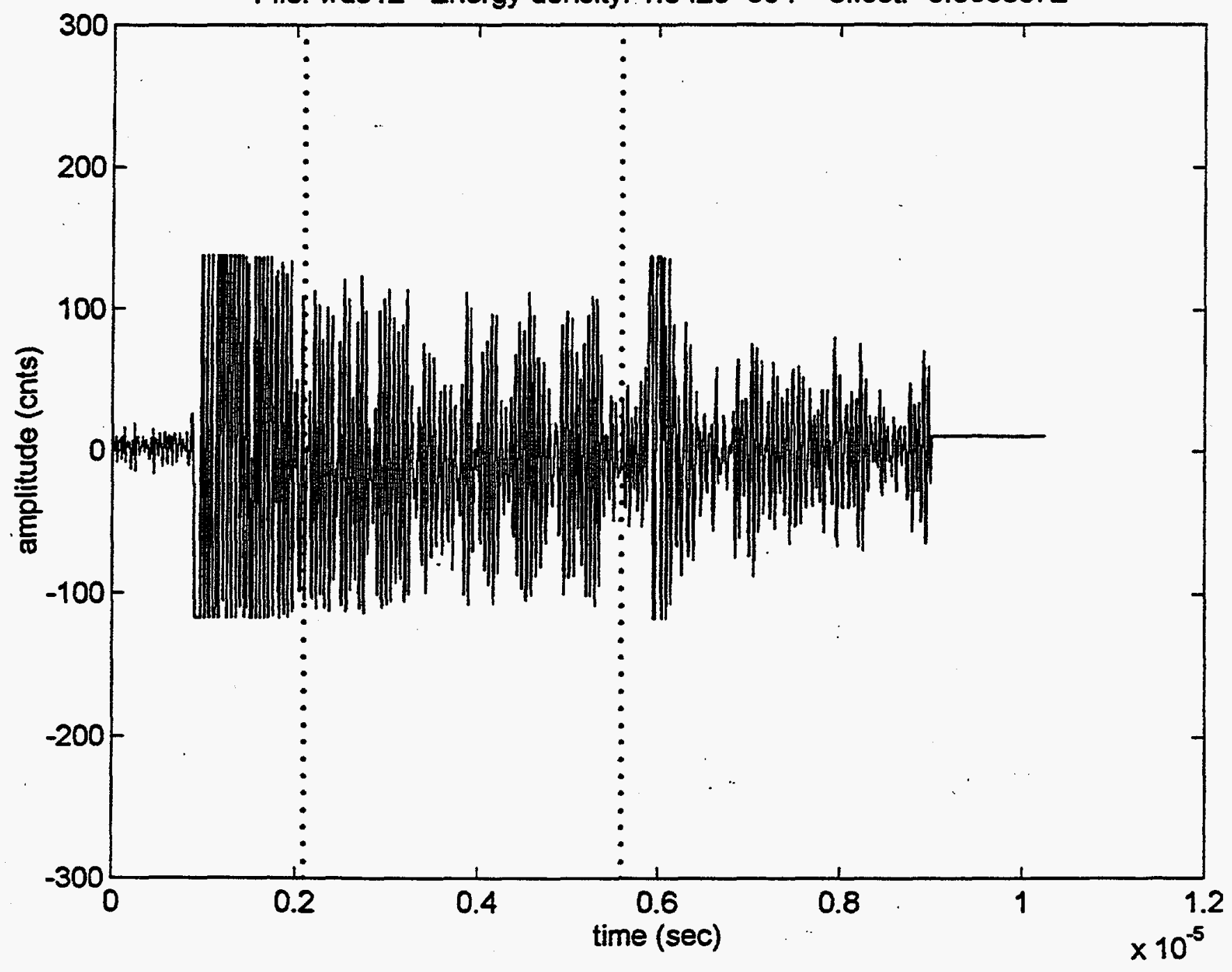


File: wd313 Energy density: $1.967 e+004$ offset: -0.00002148

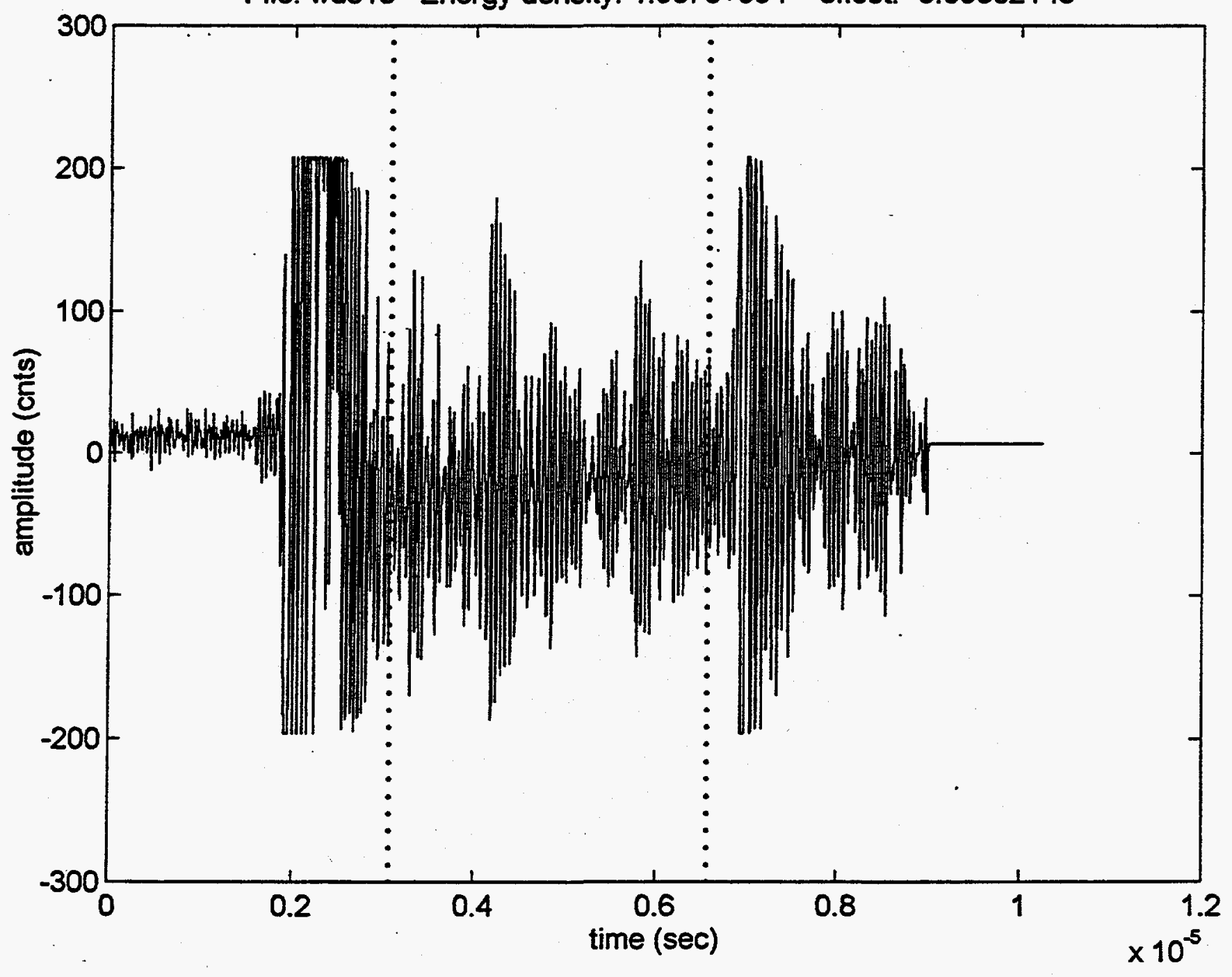


File: wd314 Energy density: $3.843 e+004$ offset: -0.00004688

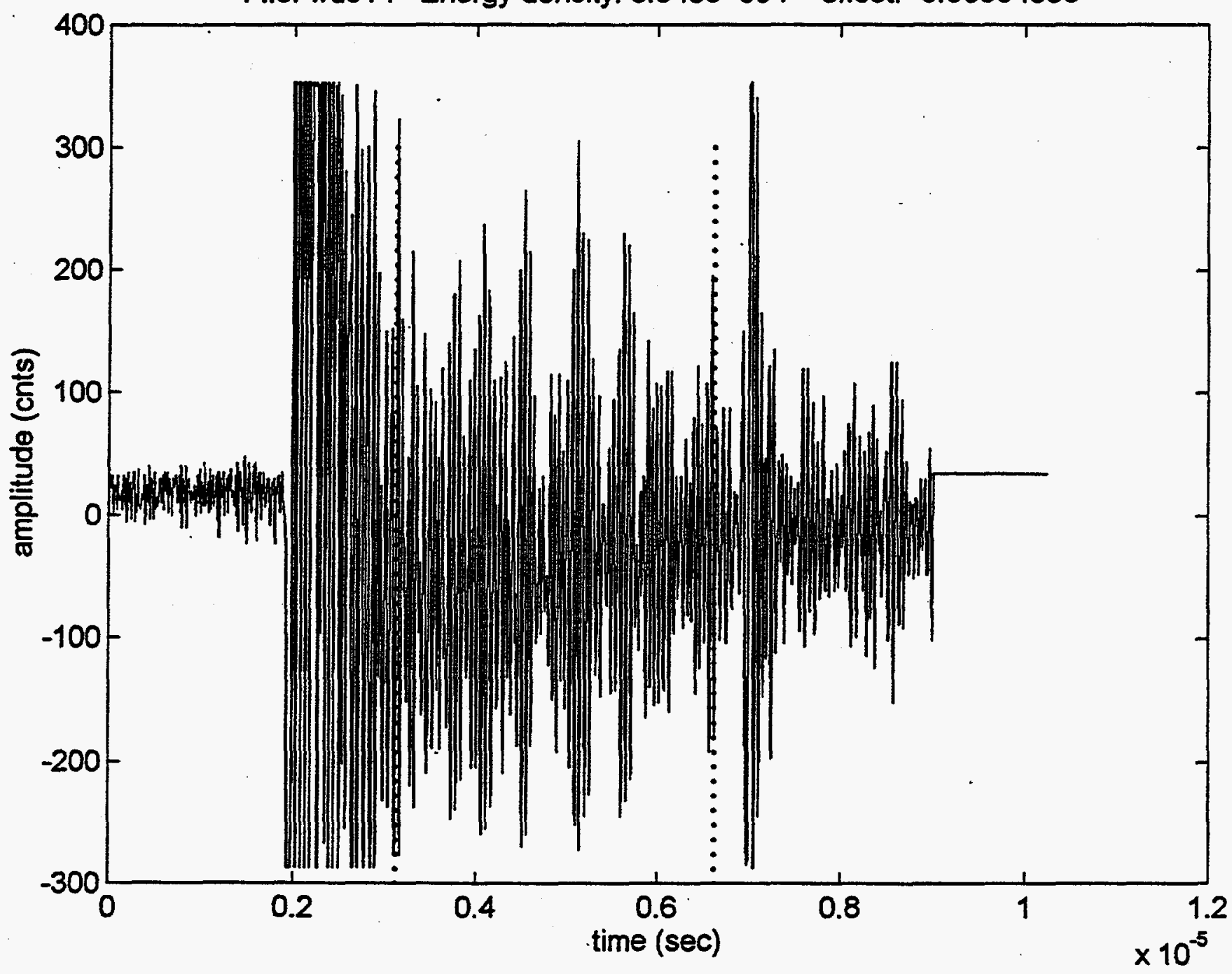


File: wd315 Energy density: $2.267 e+004$ offset: 0.00003418

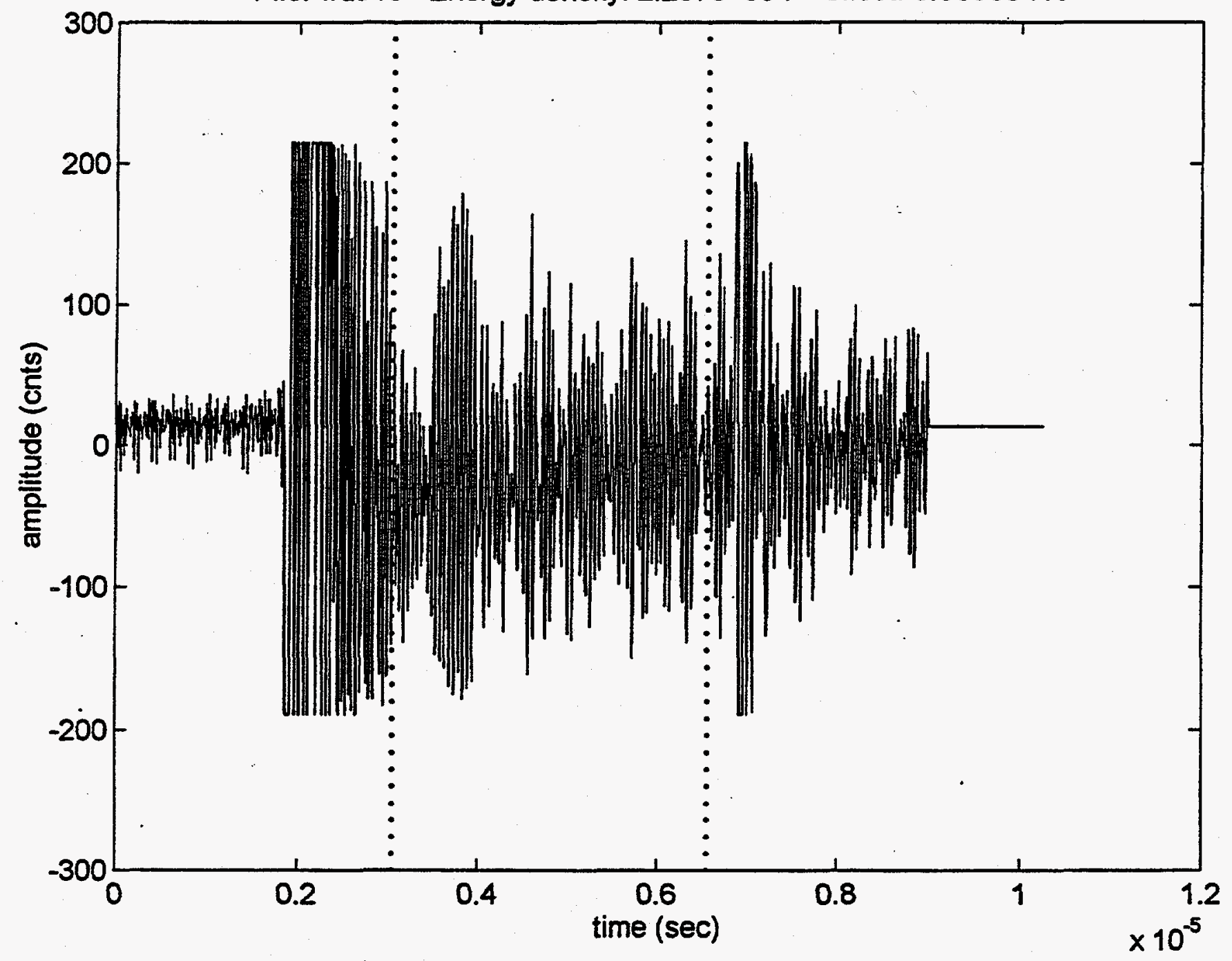




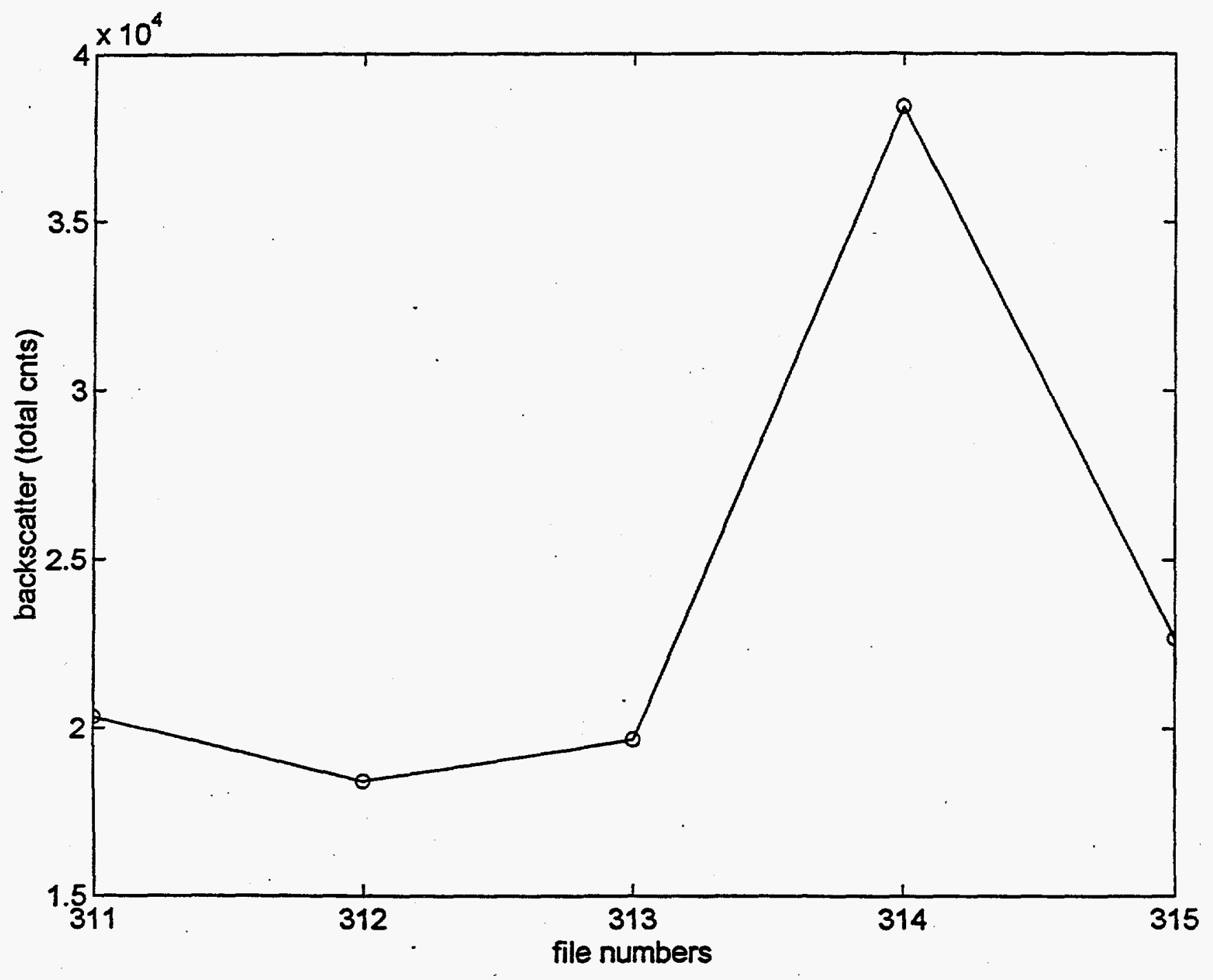


File: wd316 Energy density: $1.674 e+004$ offset: -0.00002148

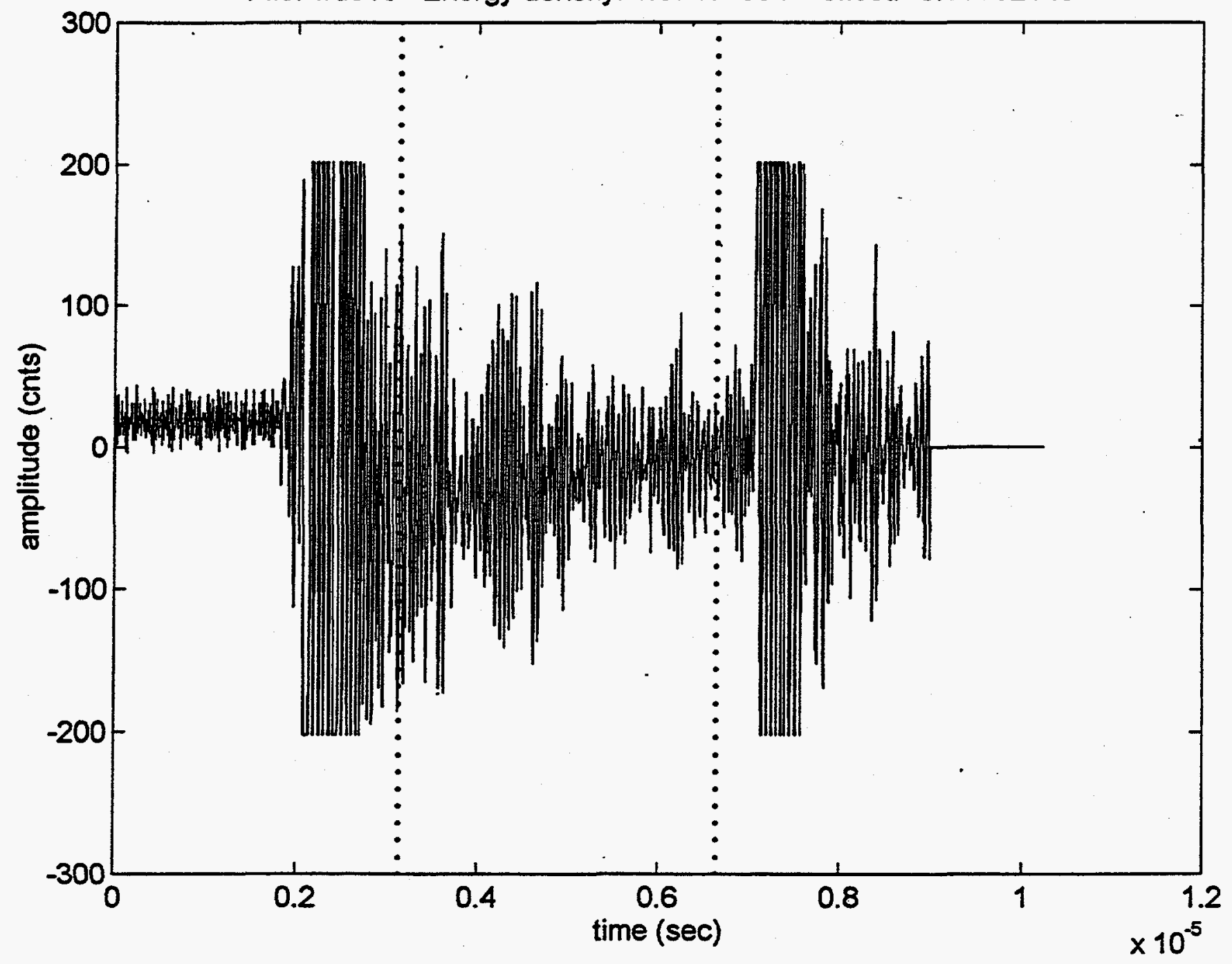


File: wd317 Energy density: $1.678 e+004$ offset: -0.00002734

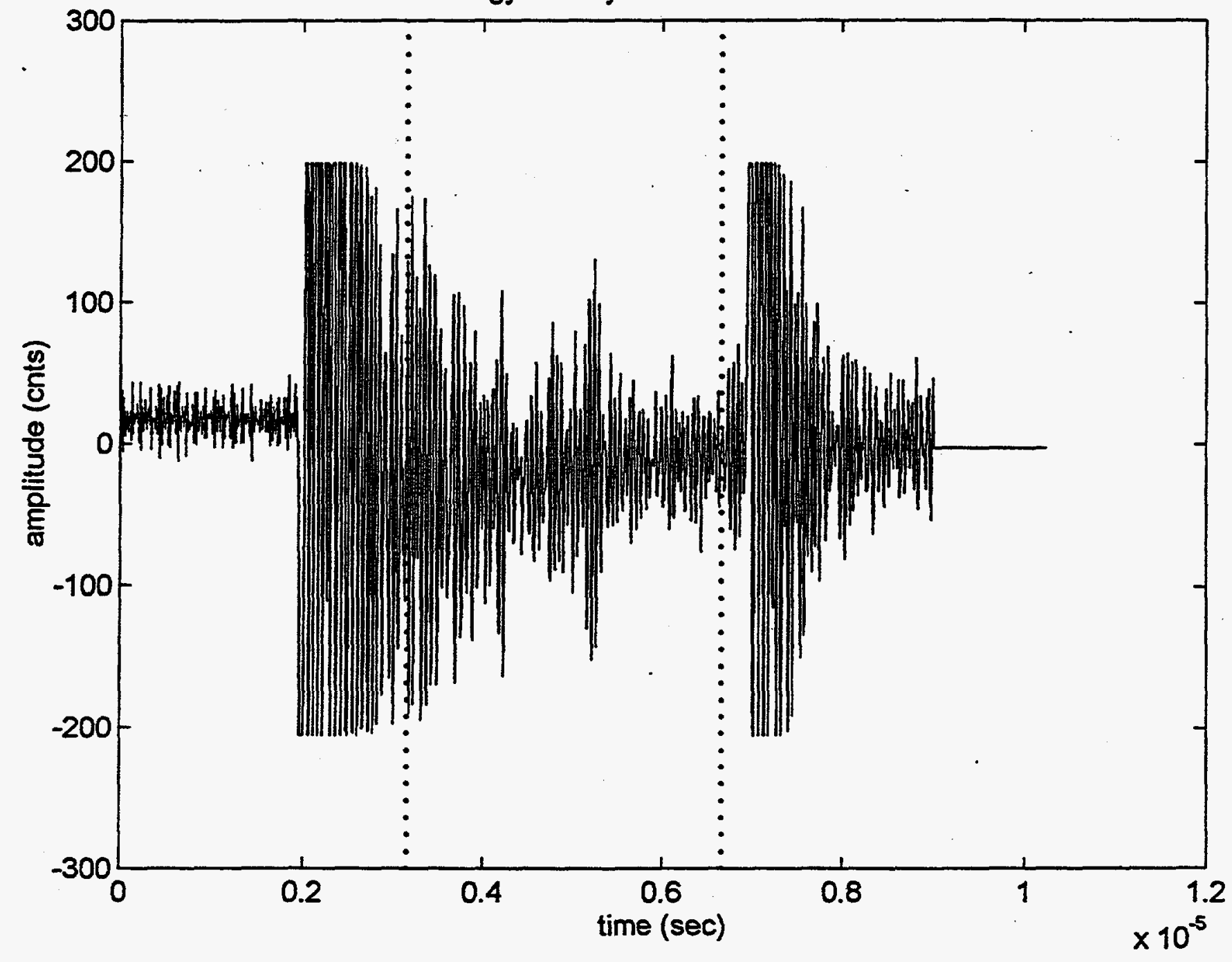


File: wd318 Energy density: $1.526 \mathrm{e}+004$ offset: 0.00001758

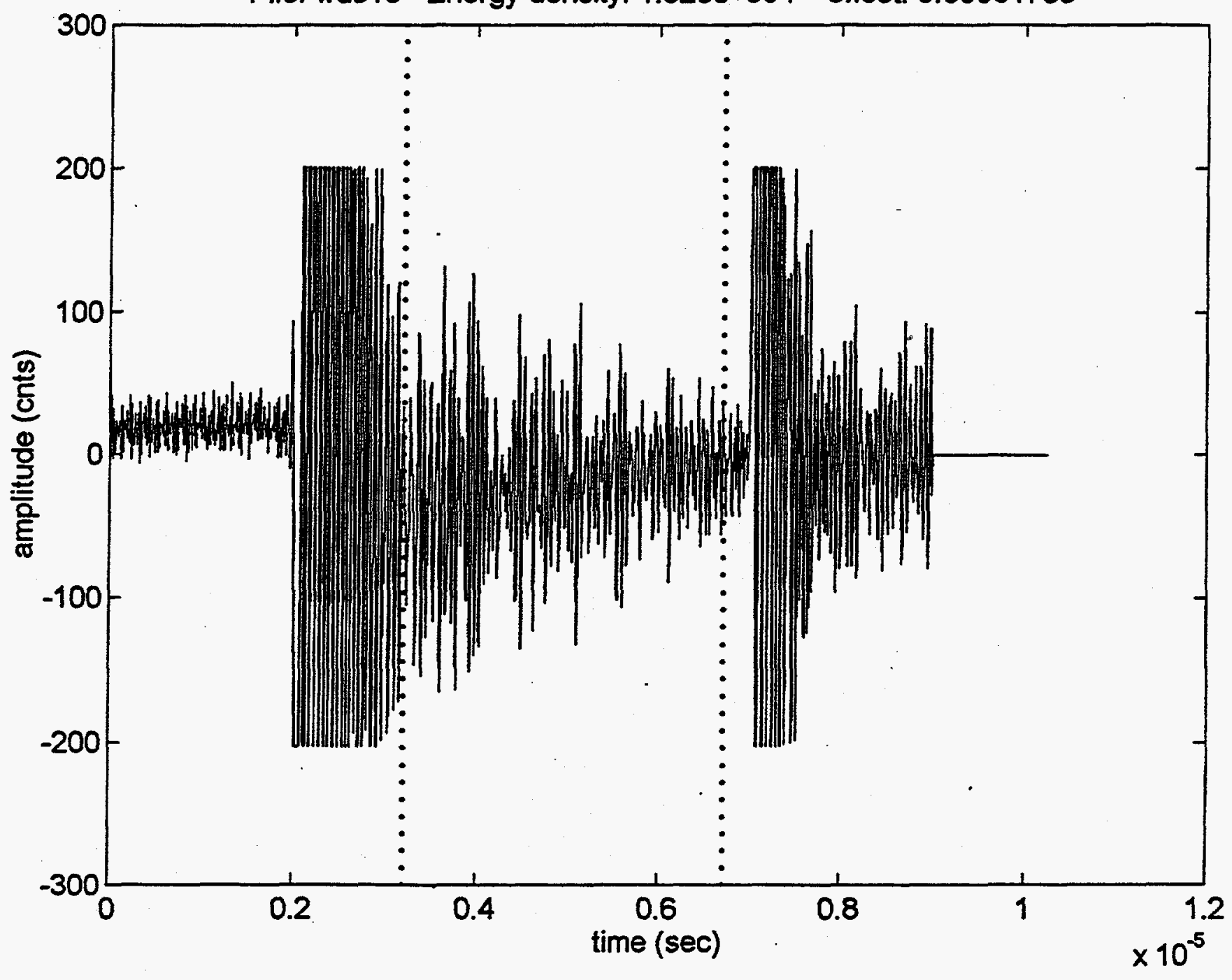


File: wd319 Energy density: $1.913 e+004$ offset: -0.00005957

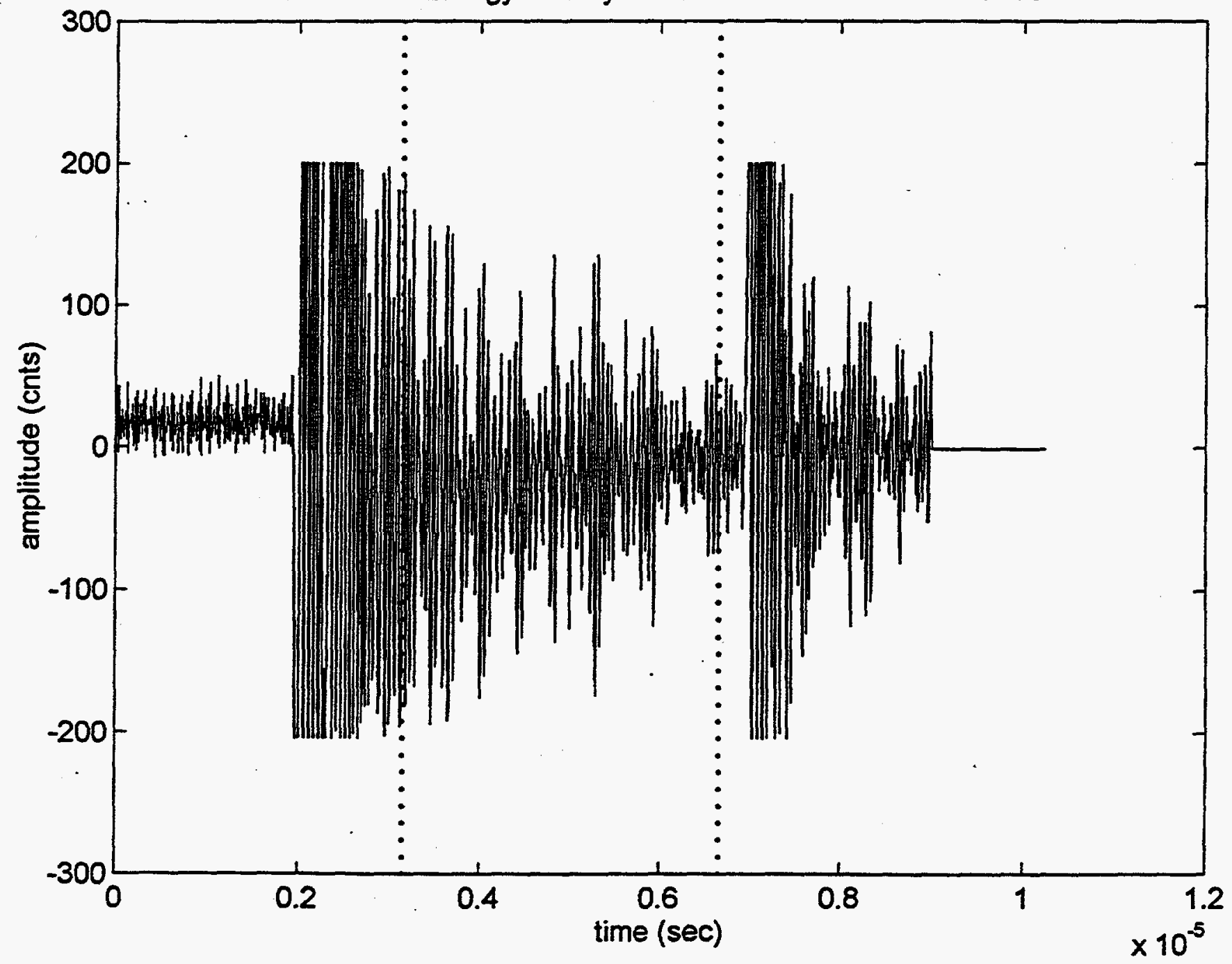


File: wd320 Energy density: $2.085 e+004$ offset: $-2.93 e-006$

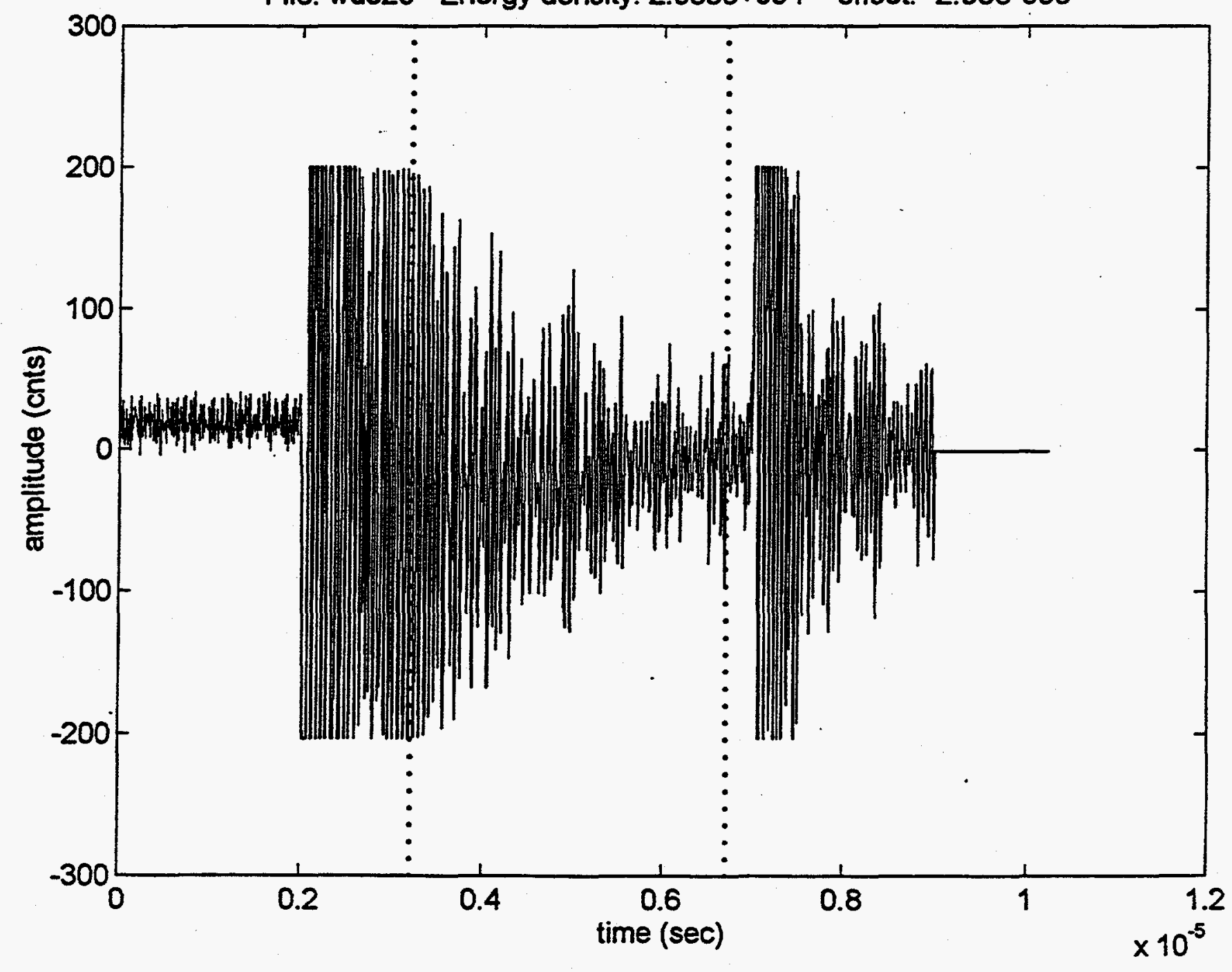




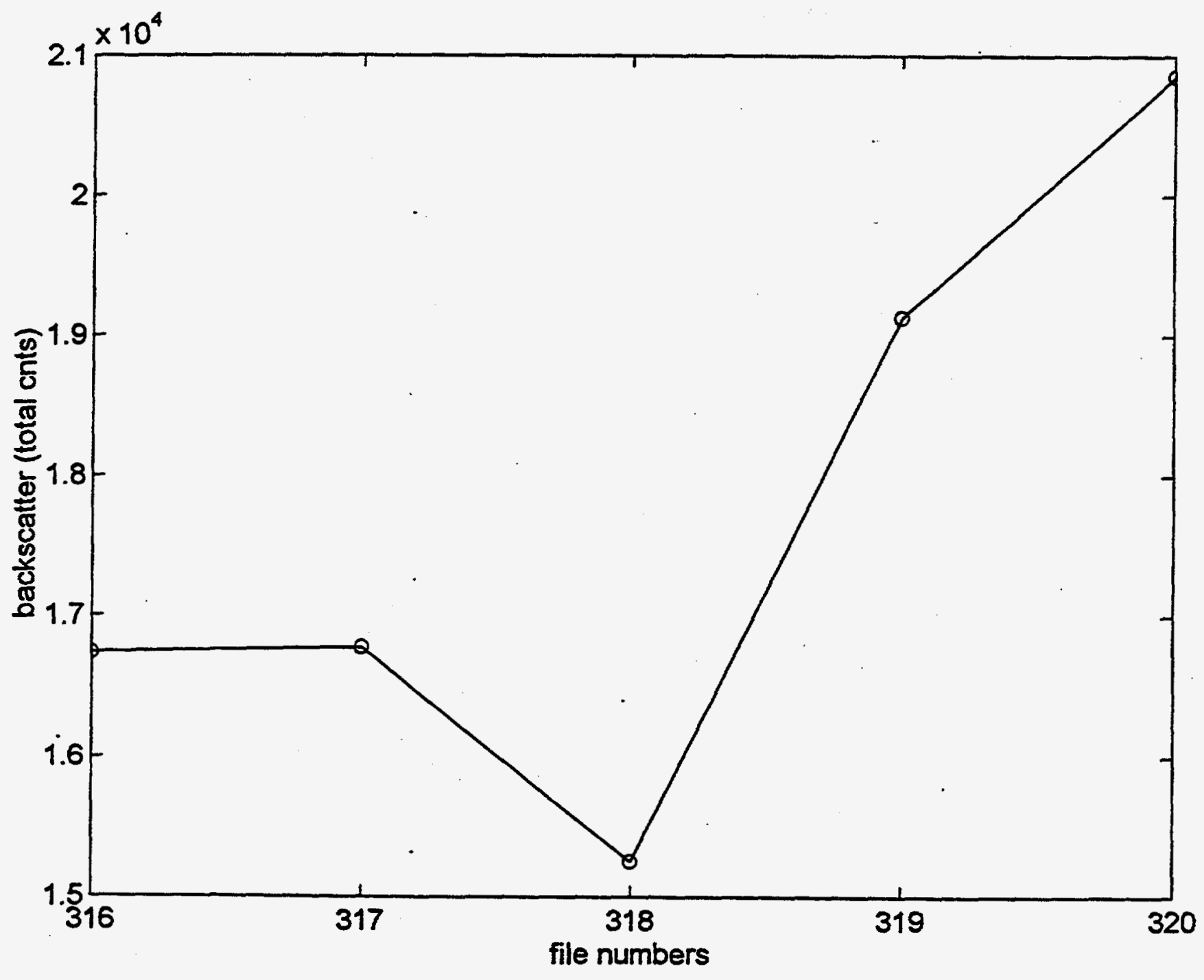


File: wd321 Energy density: $1.555 e+004$ offset: 0.00003223

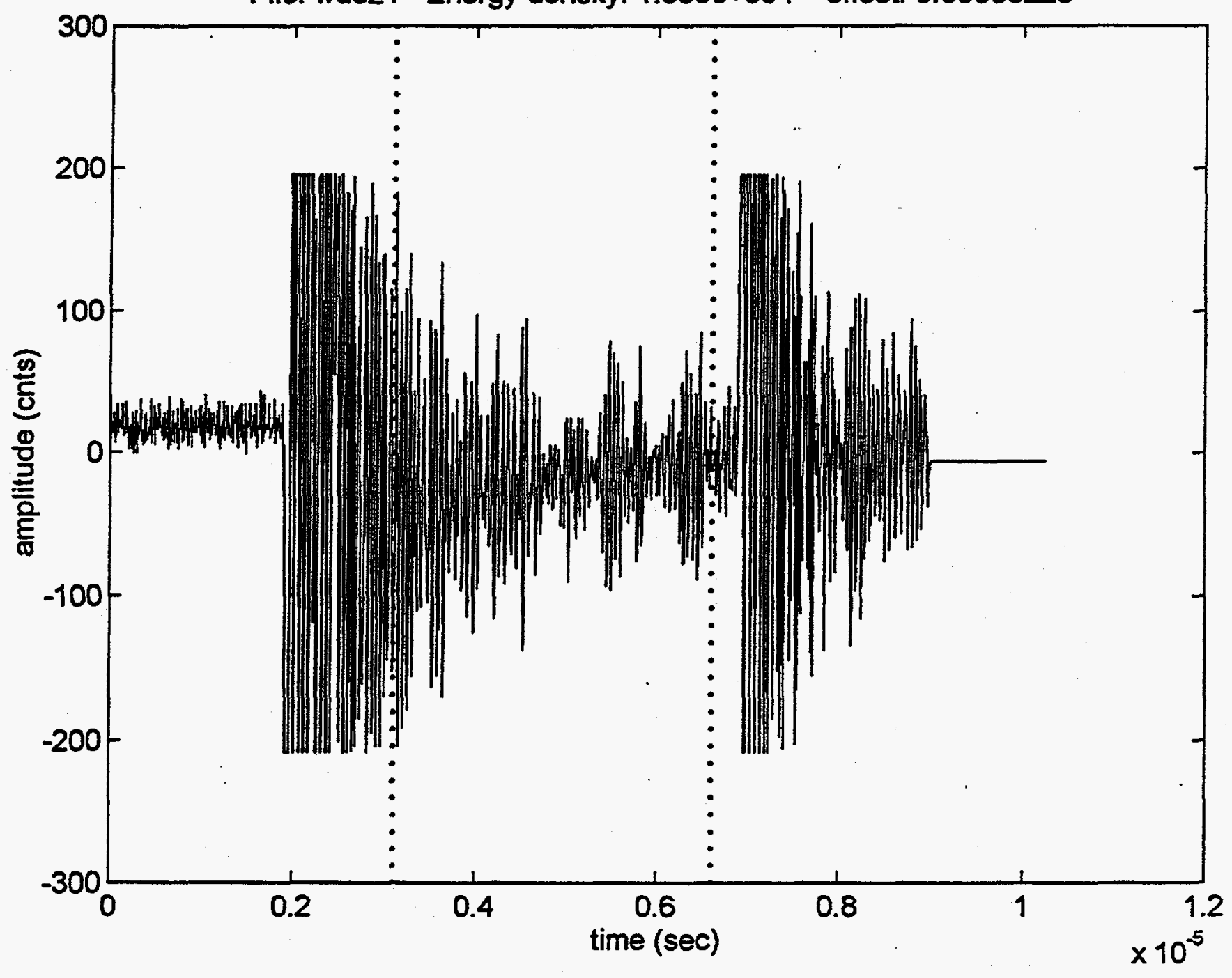


File: wd322 Energy density: $1.561 e+004$ offset: 0.00003223

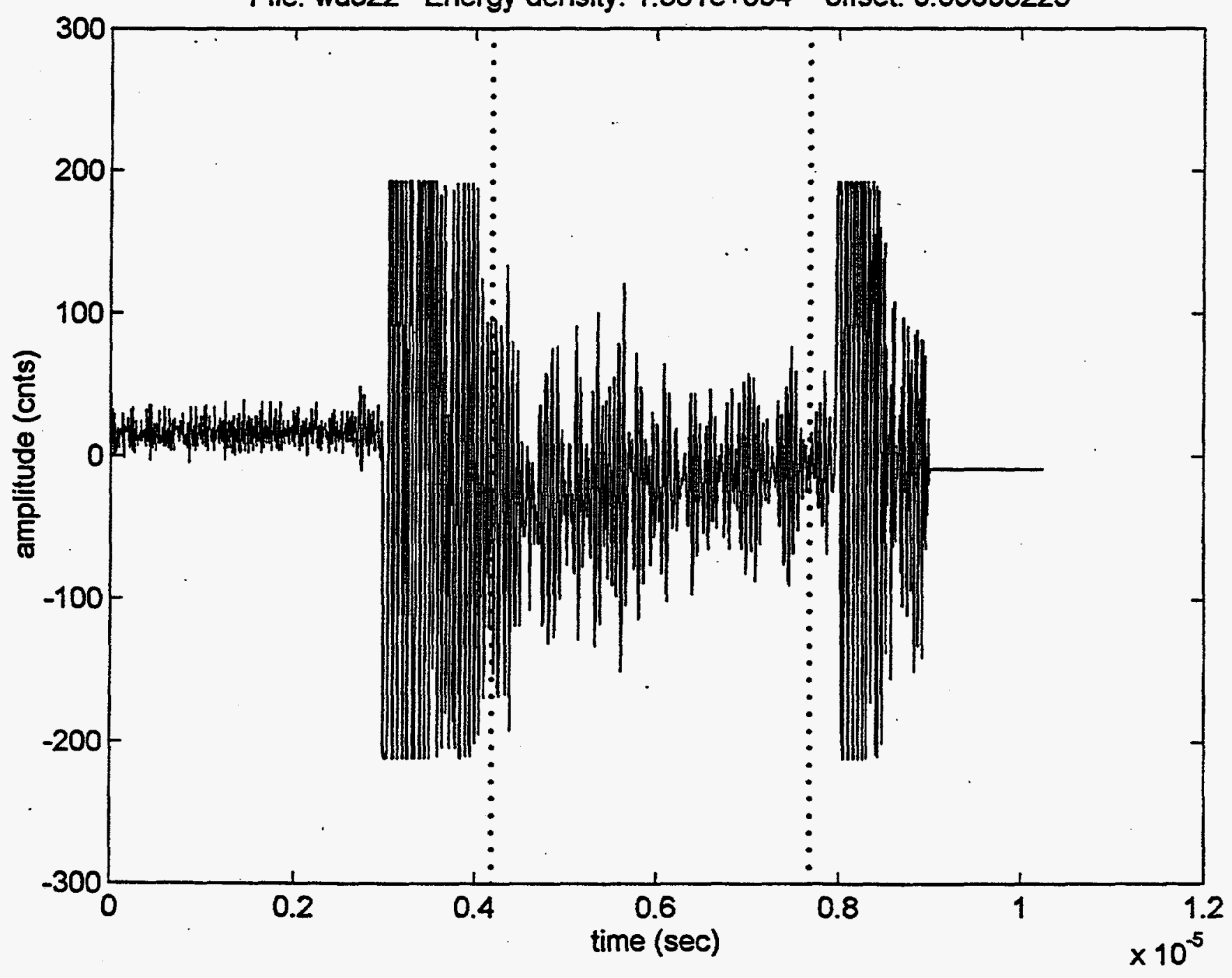


File: wd323 Energy density: $1.642 e+004$ offset: -0.00005371

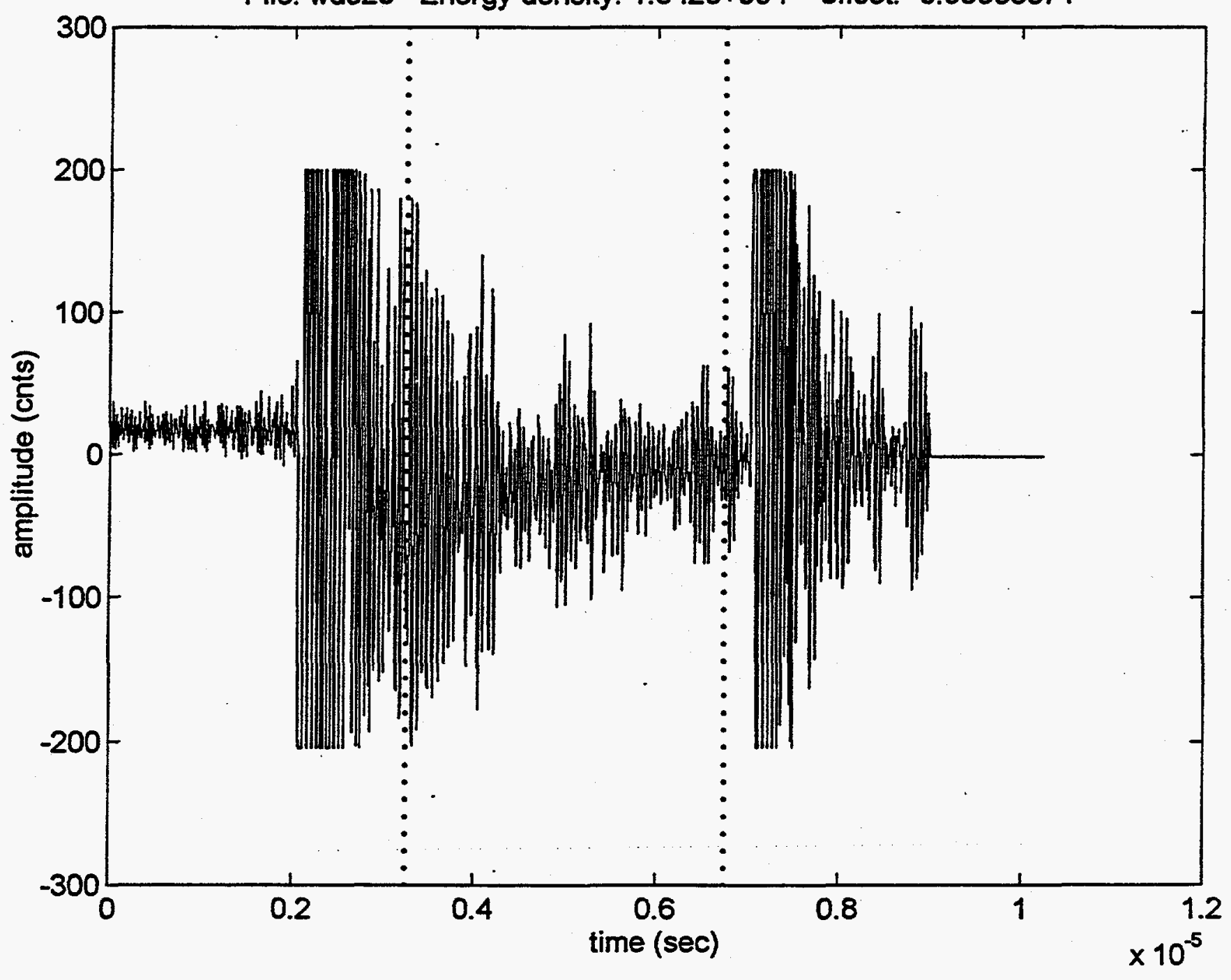




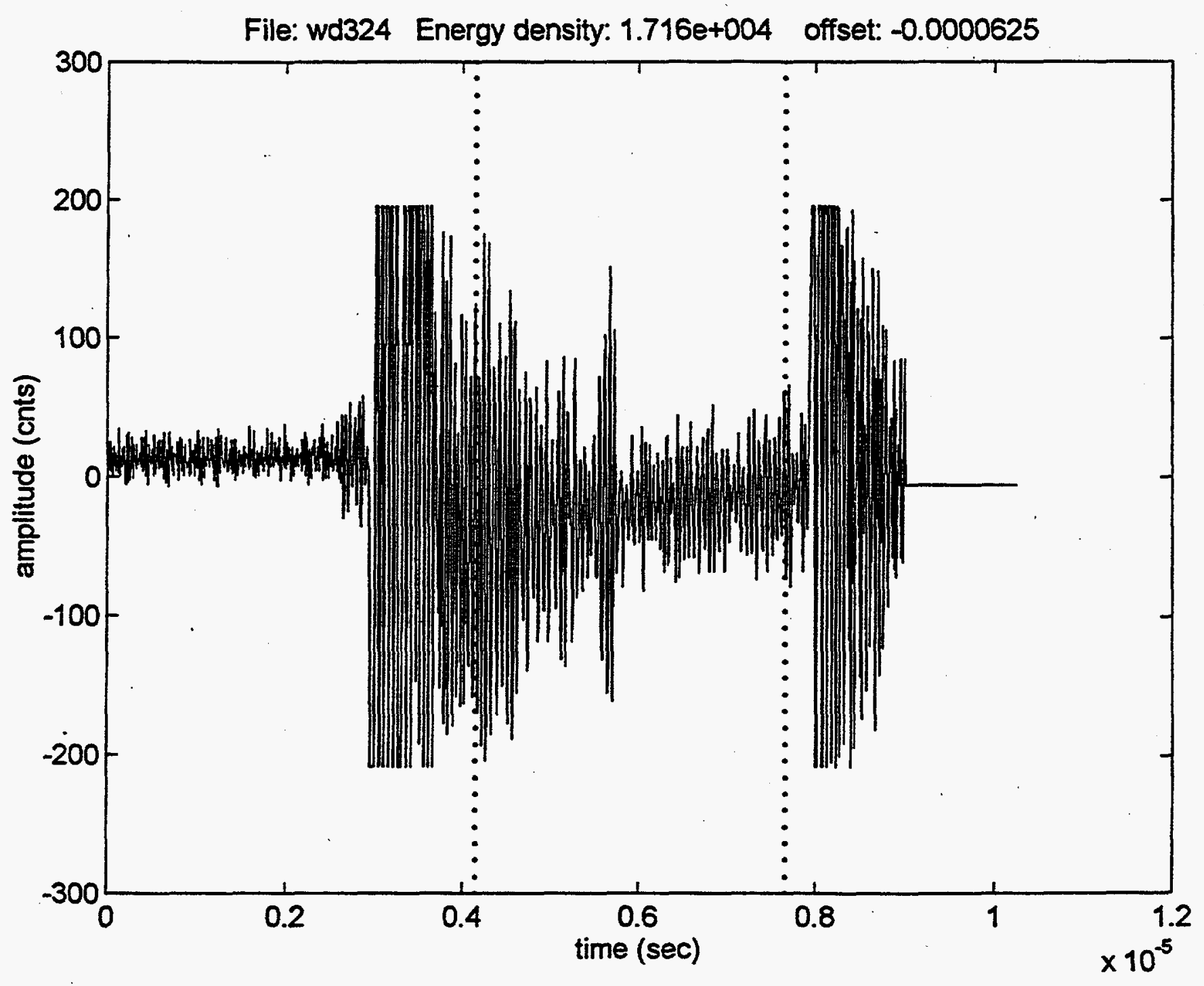


File: wd325 Energy density: 1.537 e+004 offset: -0.0000293

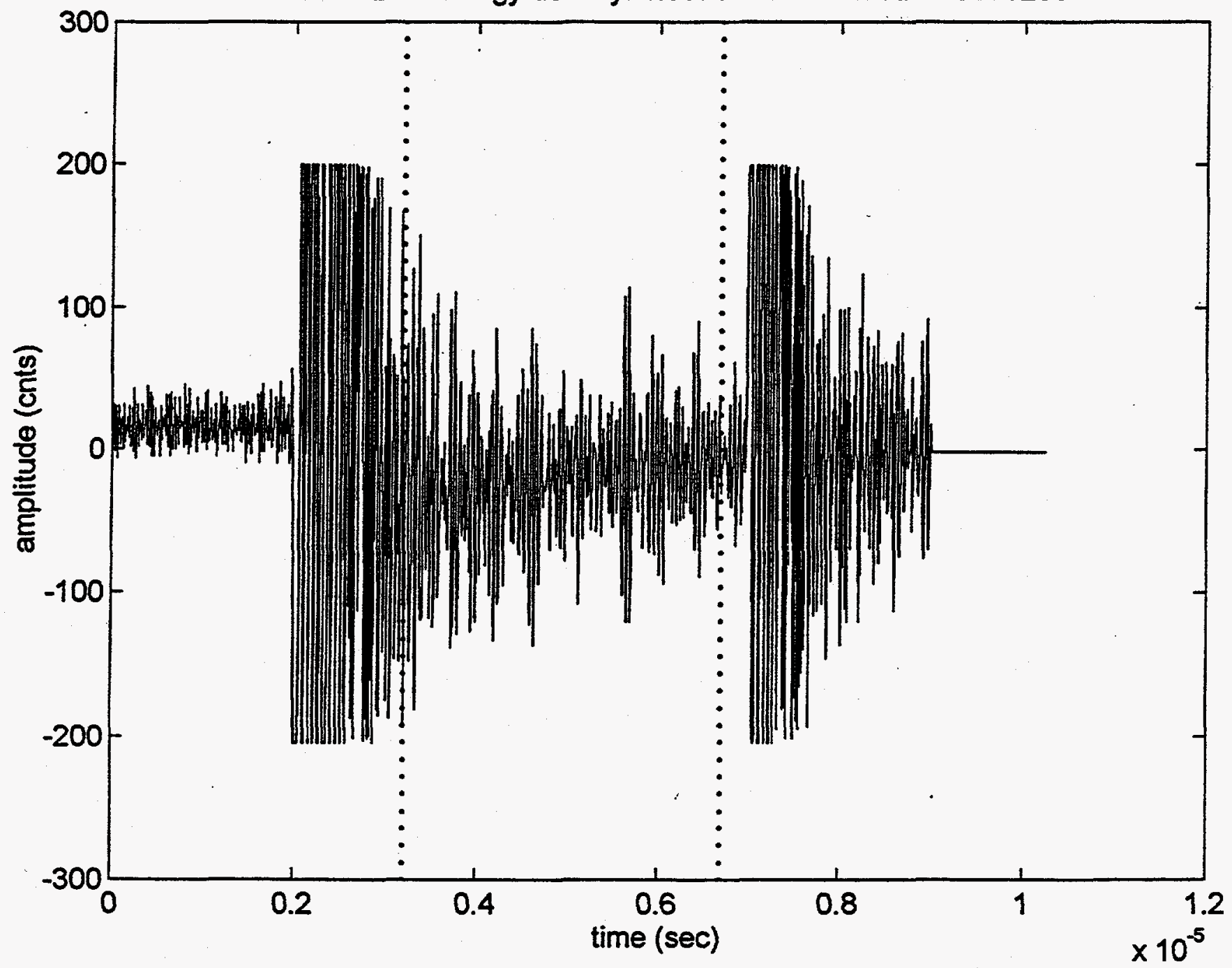




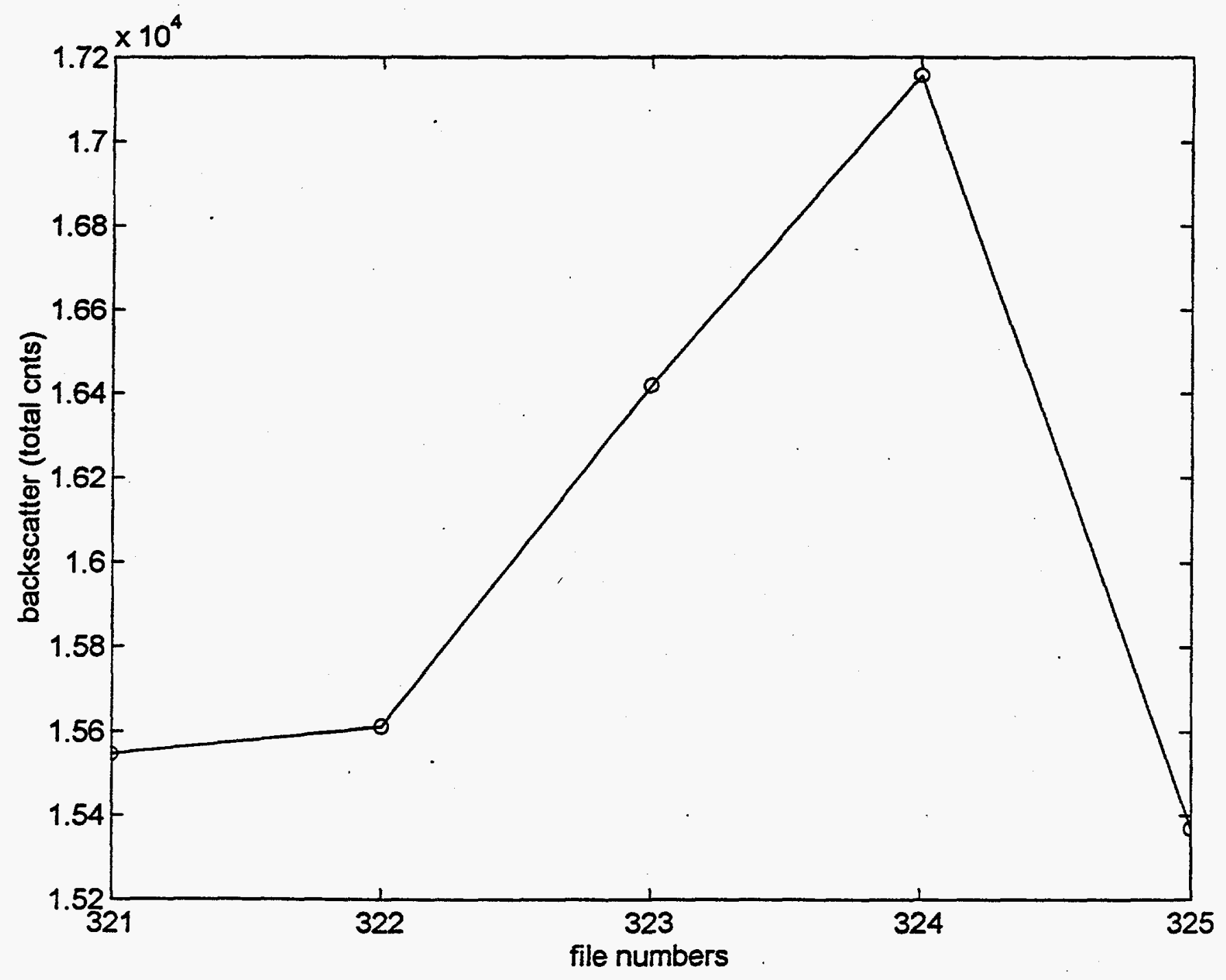


File: wd326 Energy density: $1.717 e+004$ offset: -0.00001367

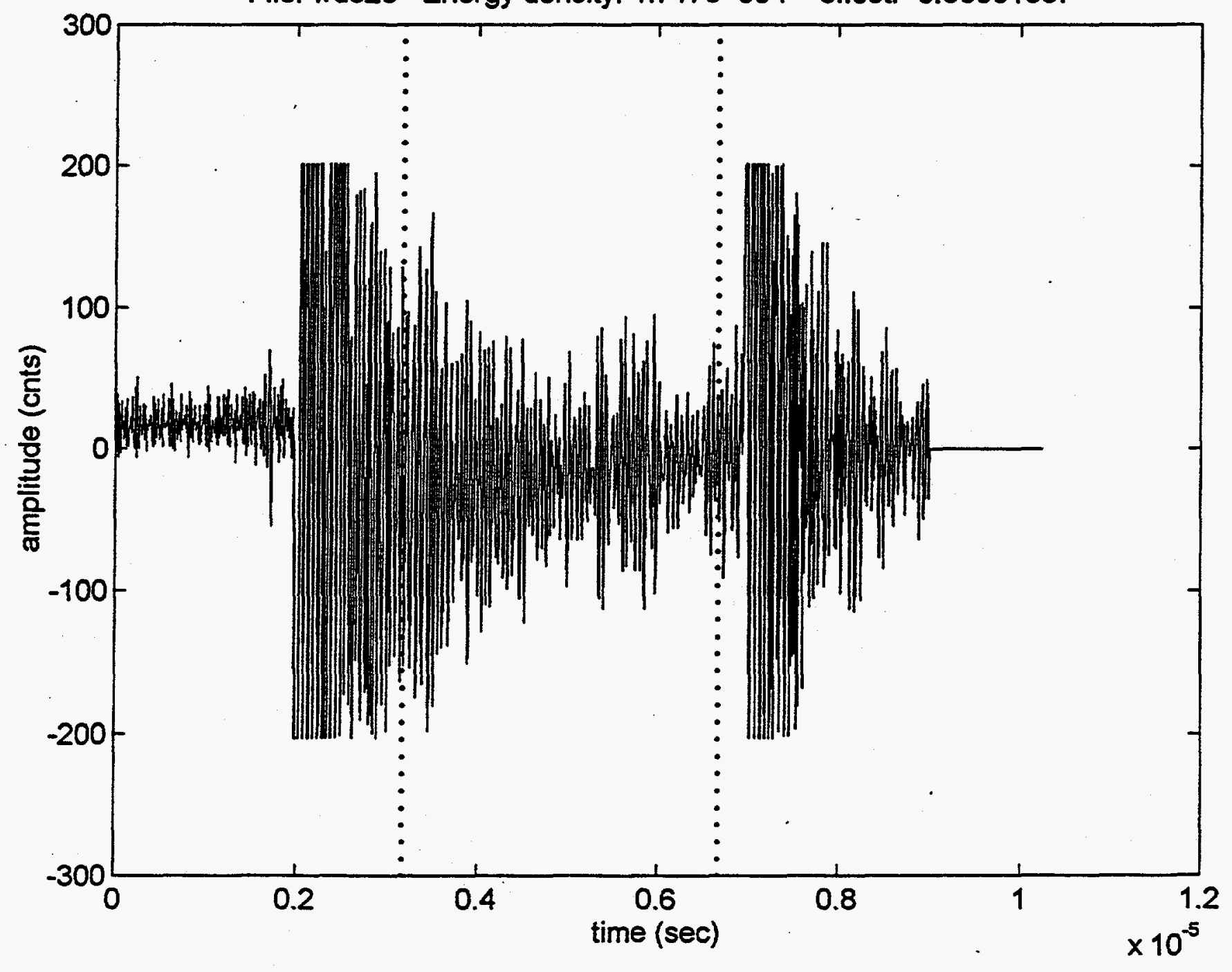


File: wd327 Energy density: $1.852 e+004$ offset: $8.789 e-006$

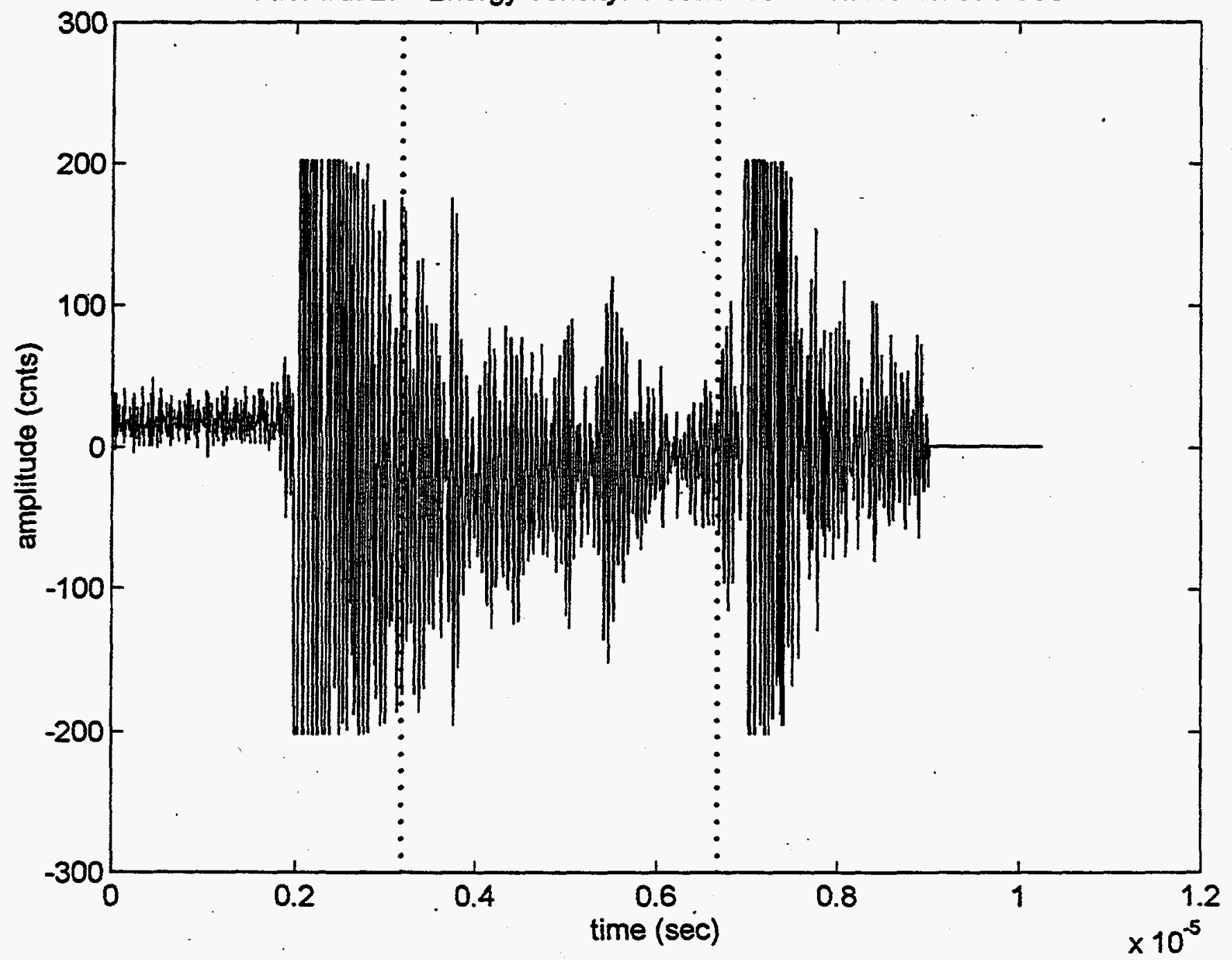



File: wd329 Energy density: $1.772 e+004$ offset: -0.00002344




File: wd330 Energy density: $1.569 e+004$ offset: -0.00002051

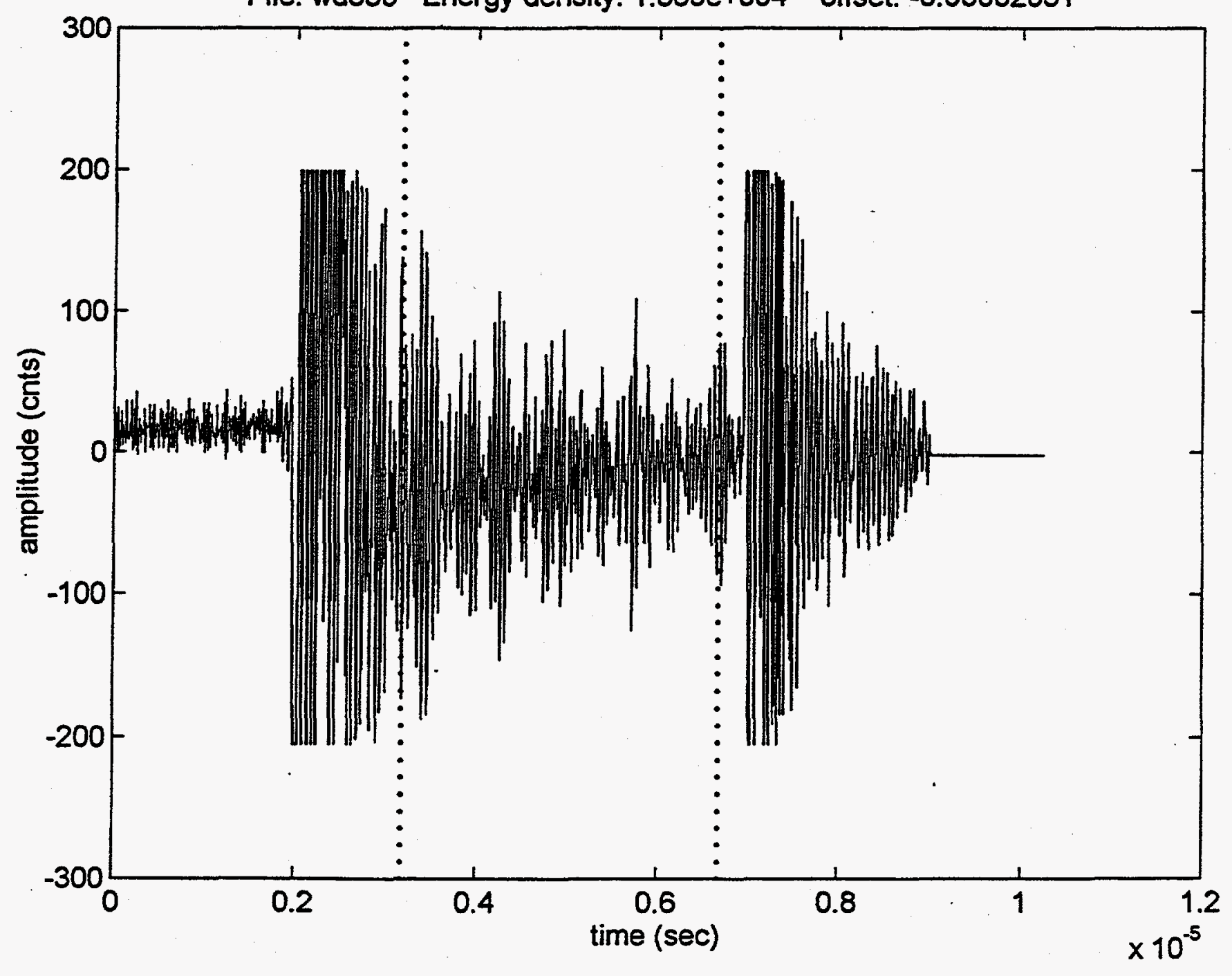




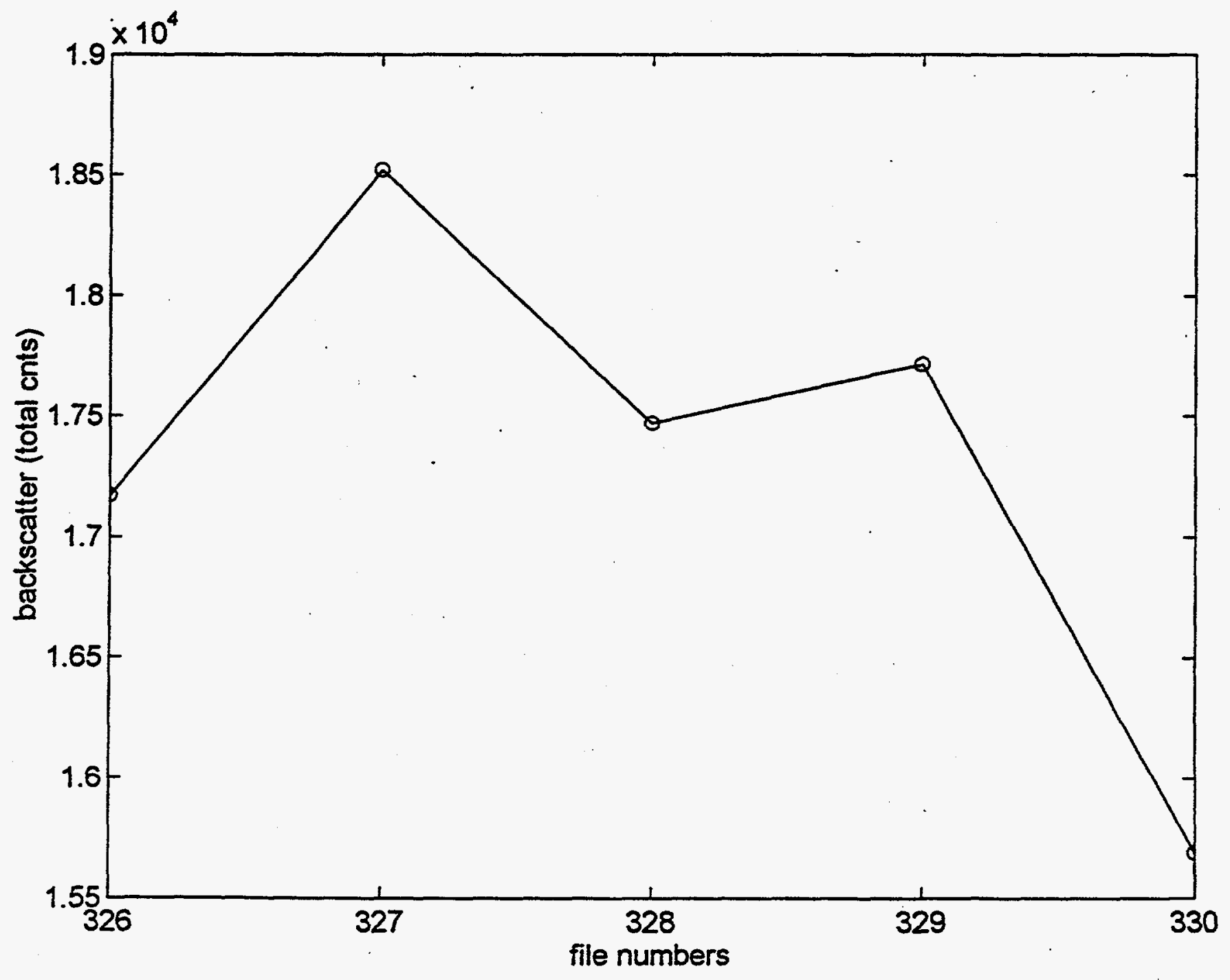


File: wd331 Energy density: 4.641 e+004 offset: -0.00003906

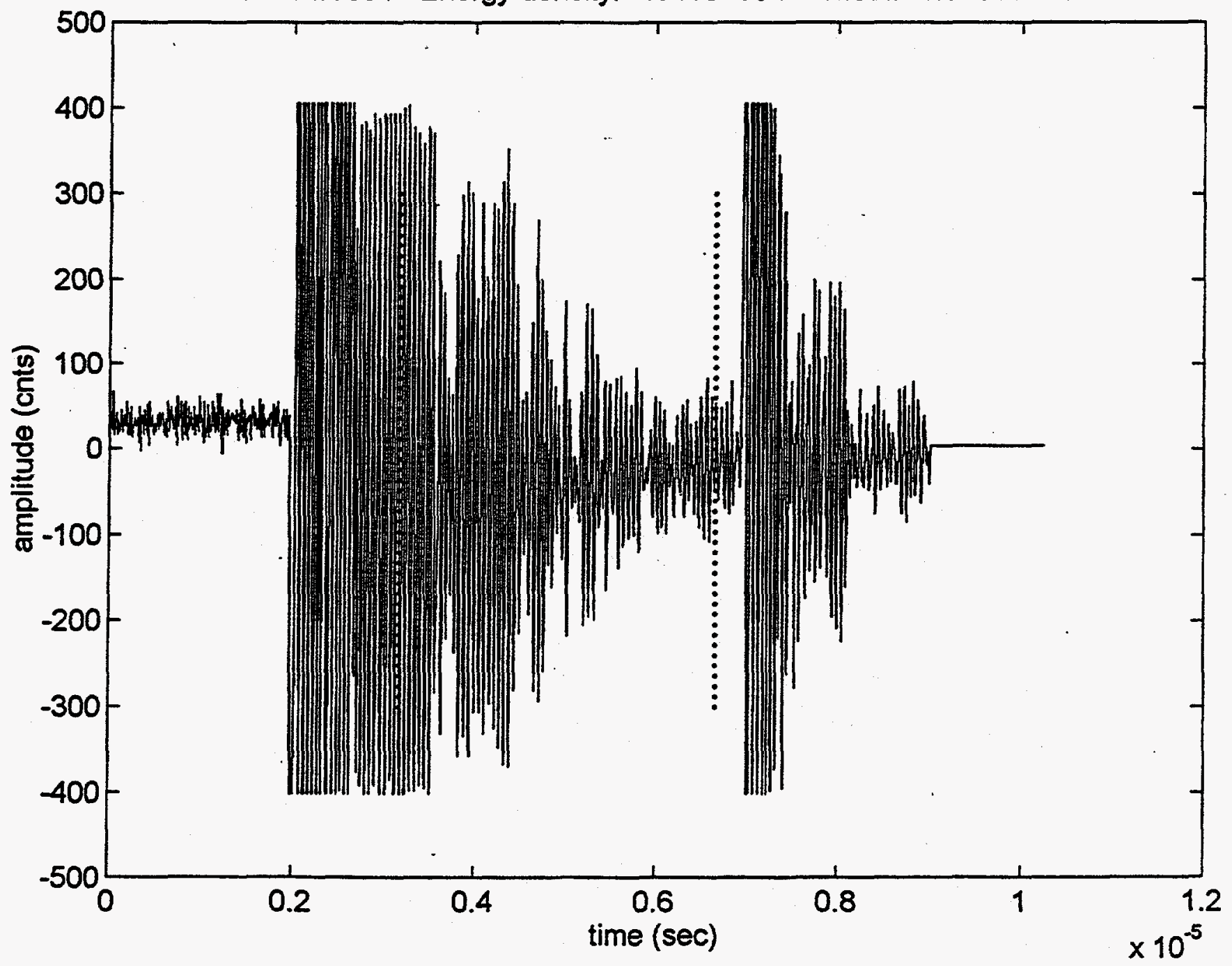


File: wd332 Energy density: $2.356 e+004$ offset: $-6.836 e-006$

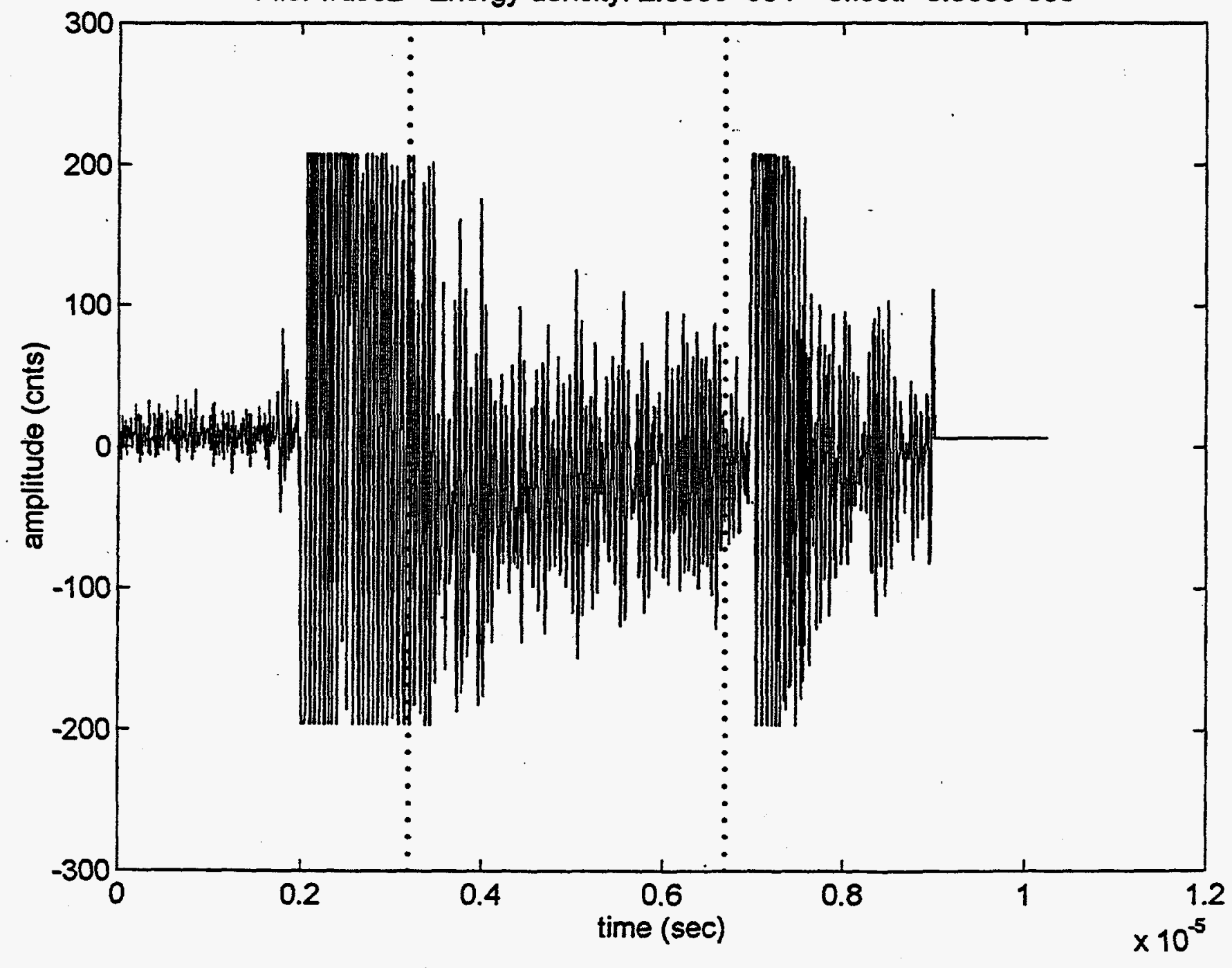


File: wd333 Energy density: $2.12 e+004$ offset: 0.00001074

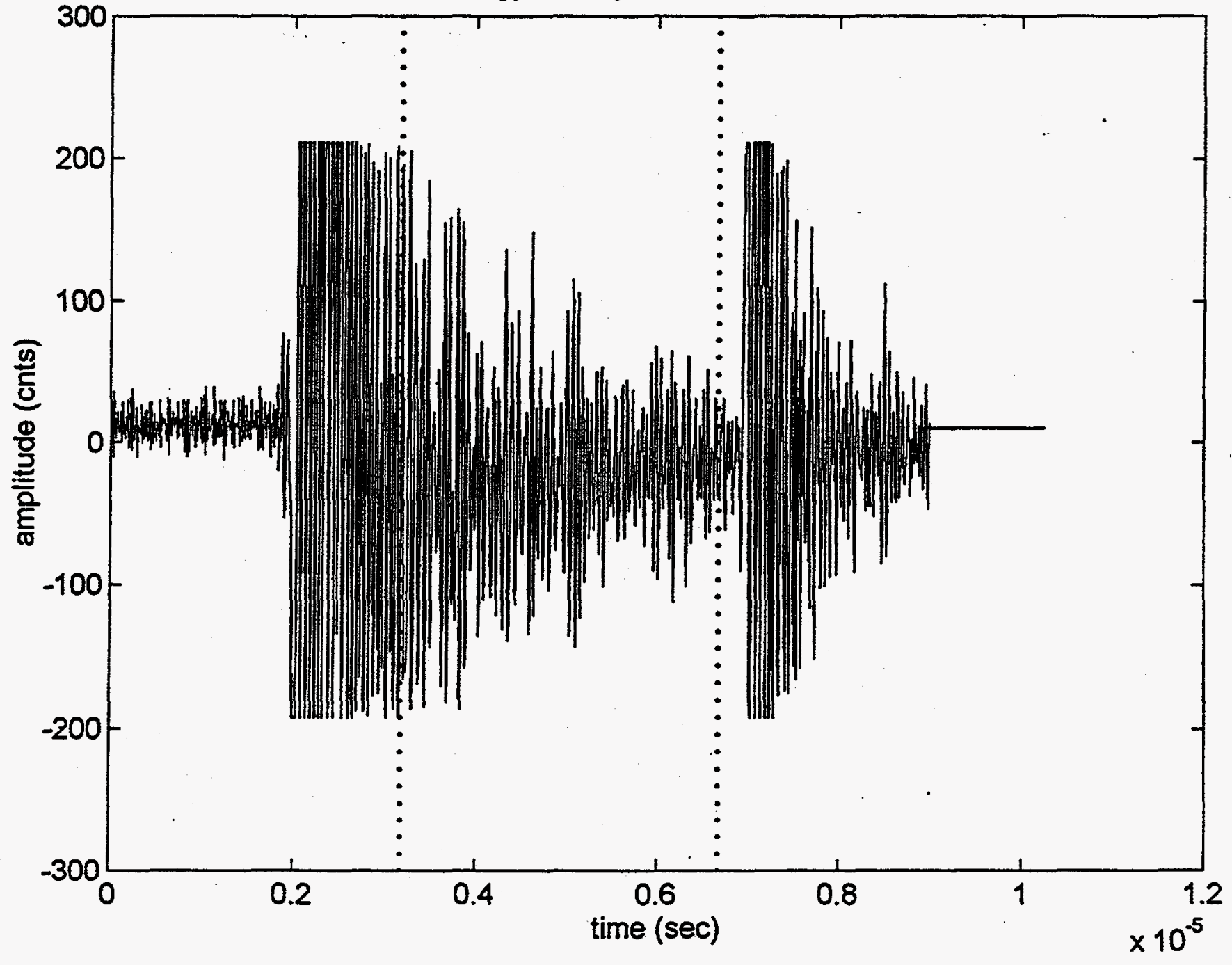


File: wd334 Energy density: $2.212 e+004$ offset: 0.00005078

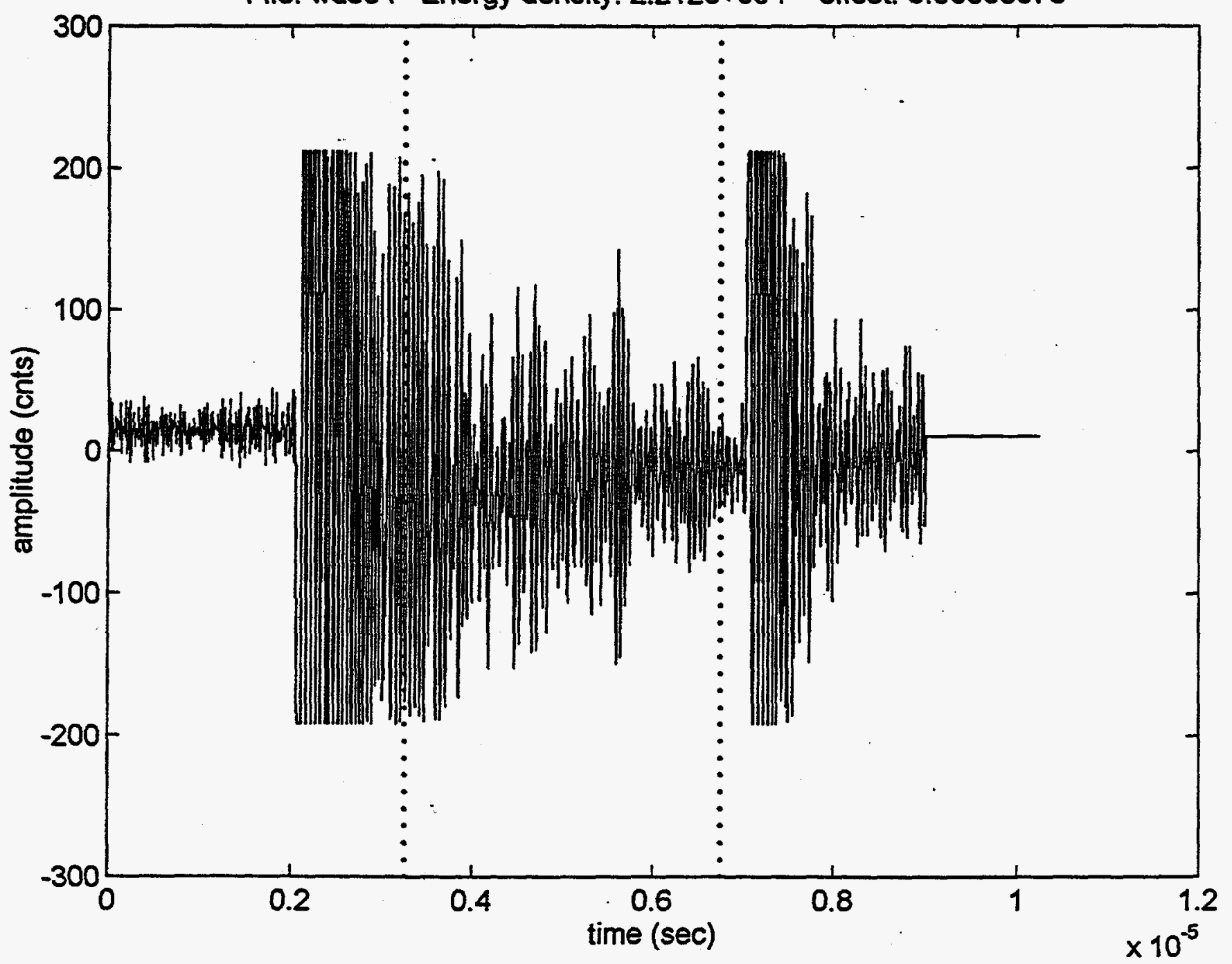


File: wd335 Energy density: $1.787 e+004$ offset: 0.00002344

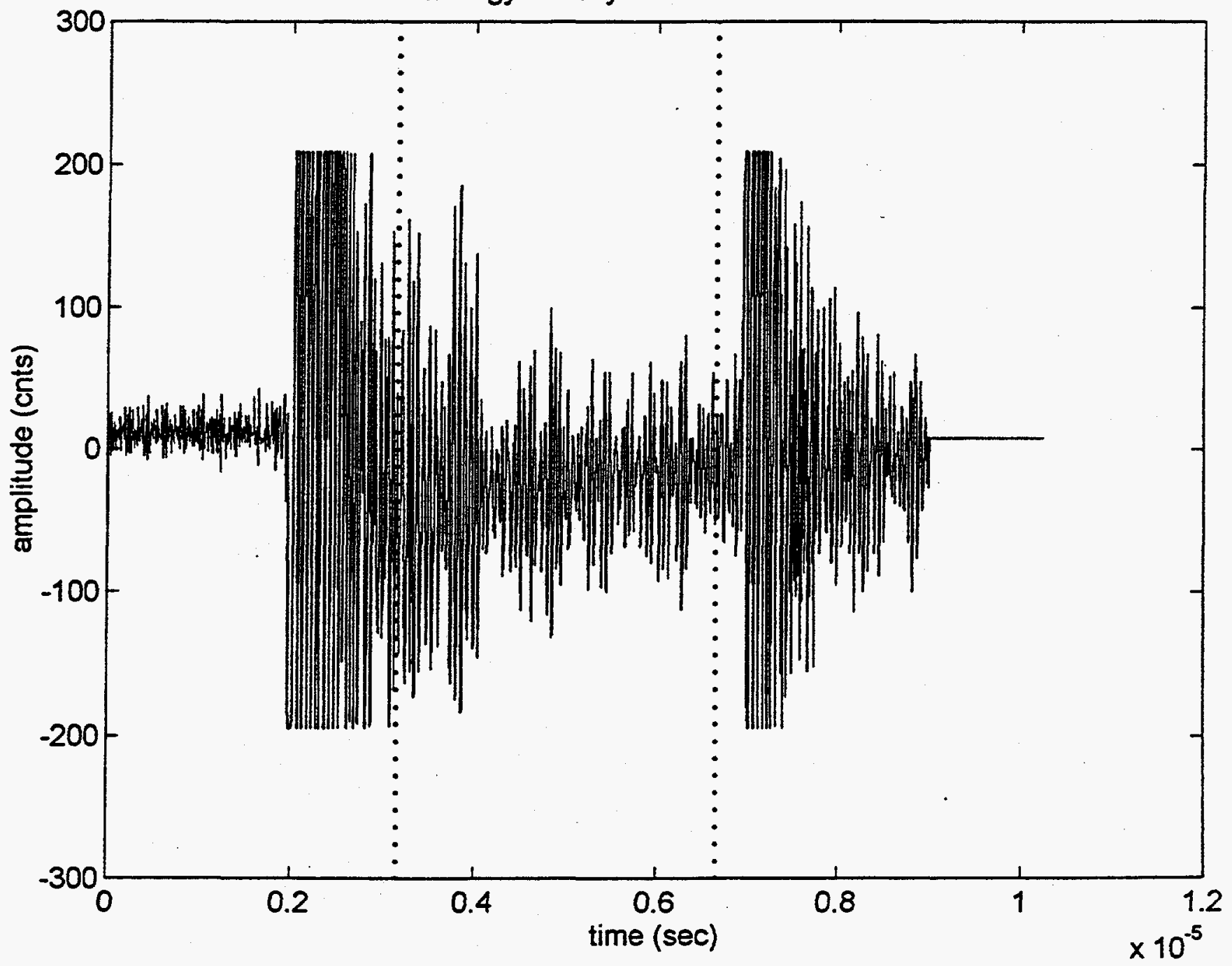




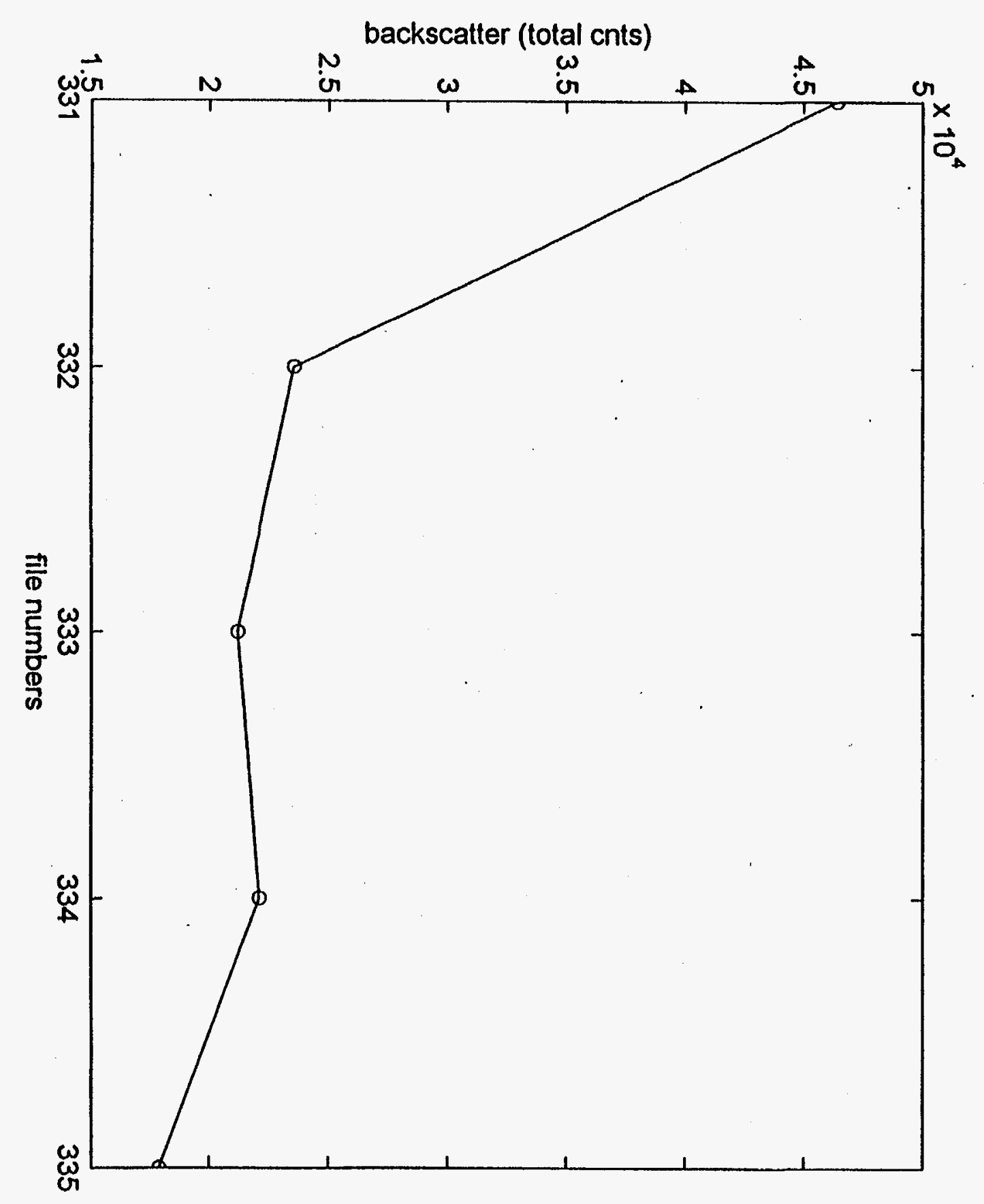


File: wd336 Energy density: $1.592 e+004$ offset: -0.00002832

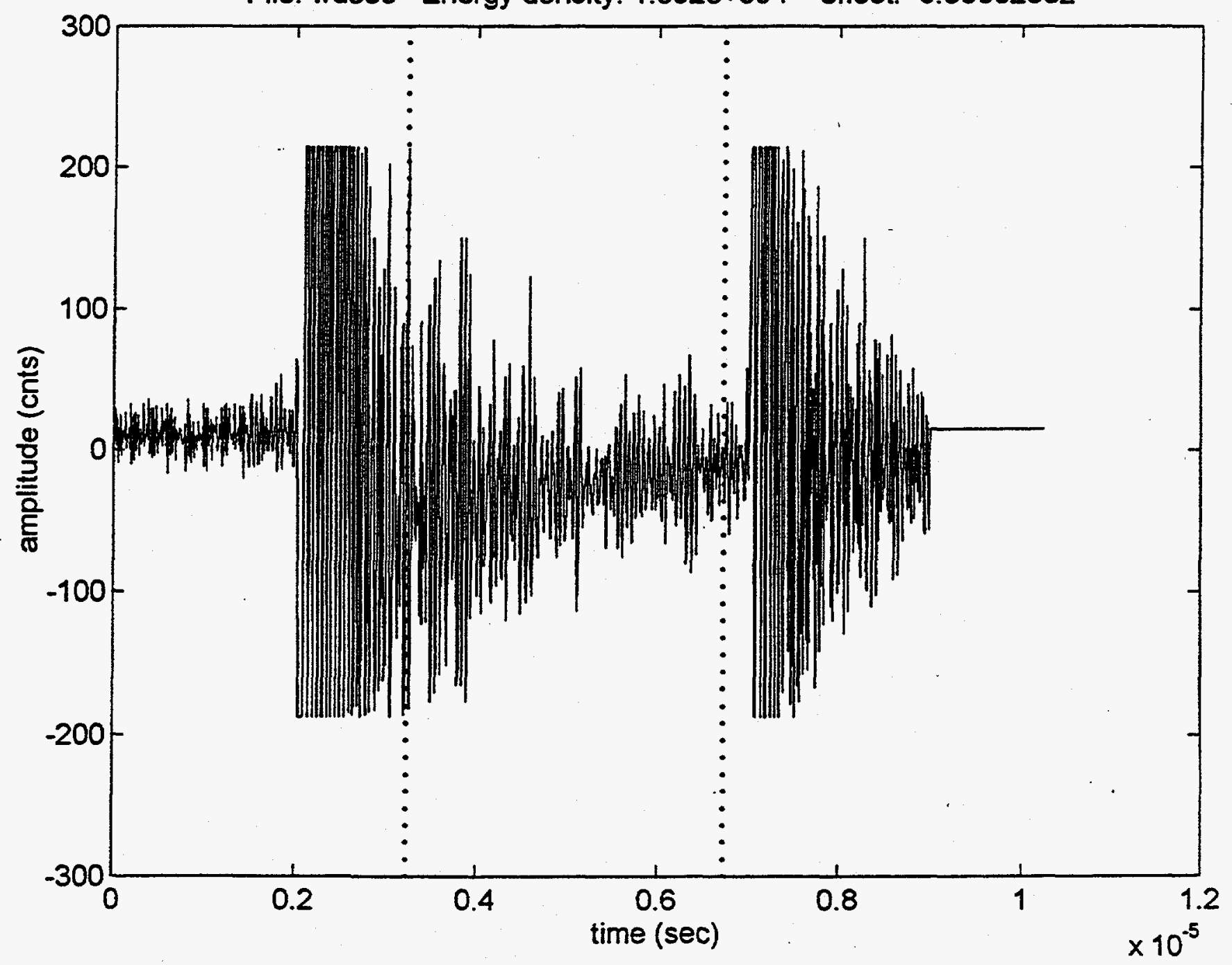


File: wd337 Energy density: $1.972 e+004$ offset: $-9.343 e-015$

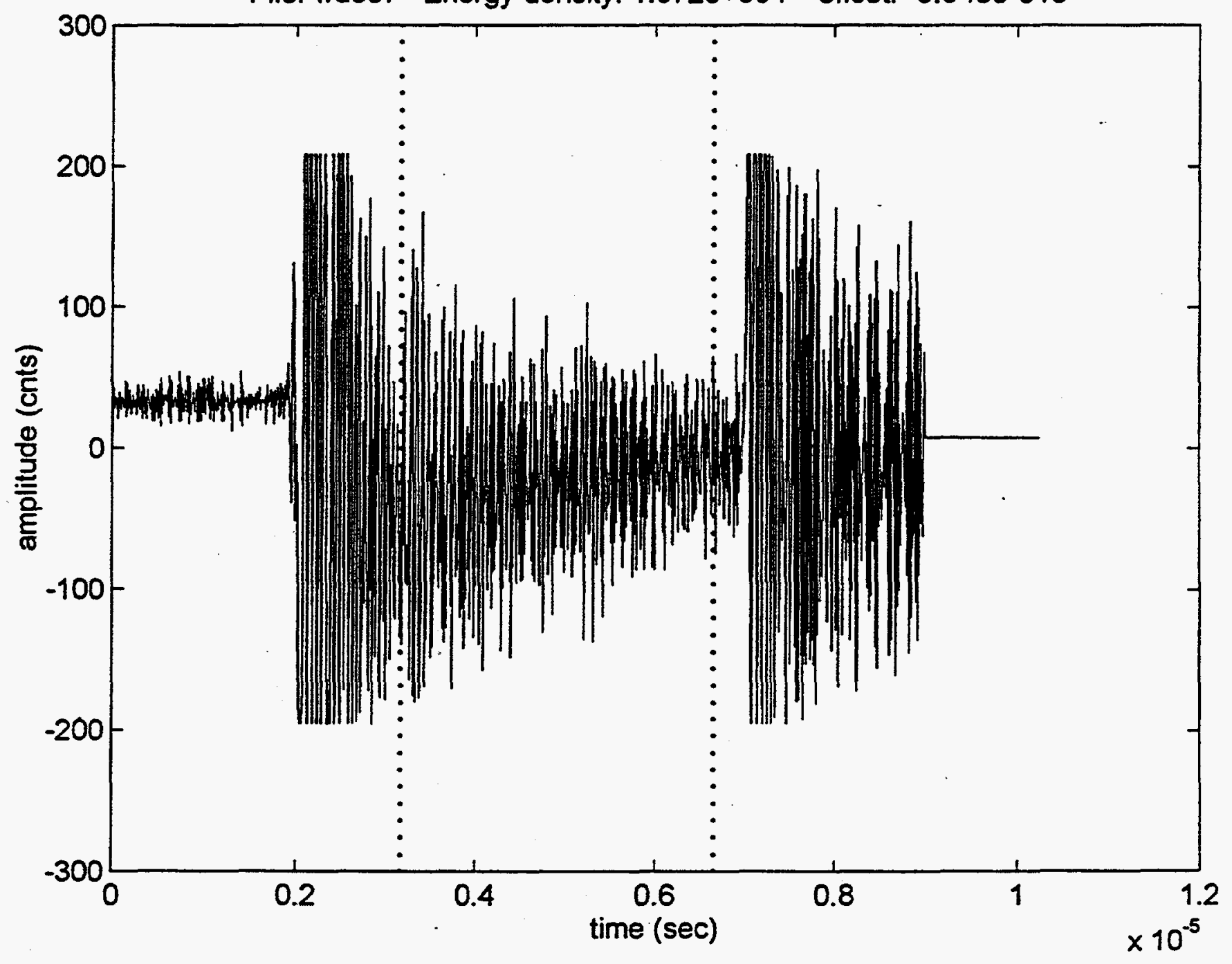


File: wd338 Energy density: $1.579 e+004$ offset: 0.0000332

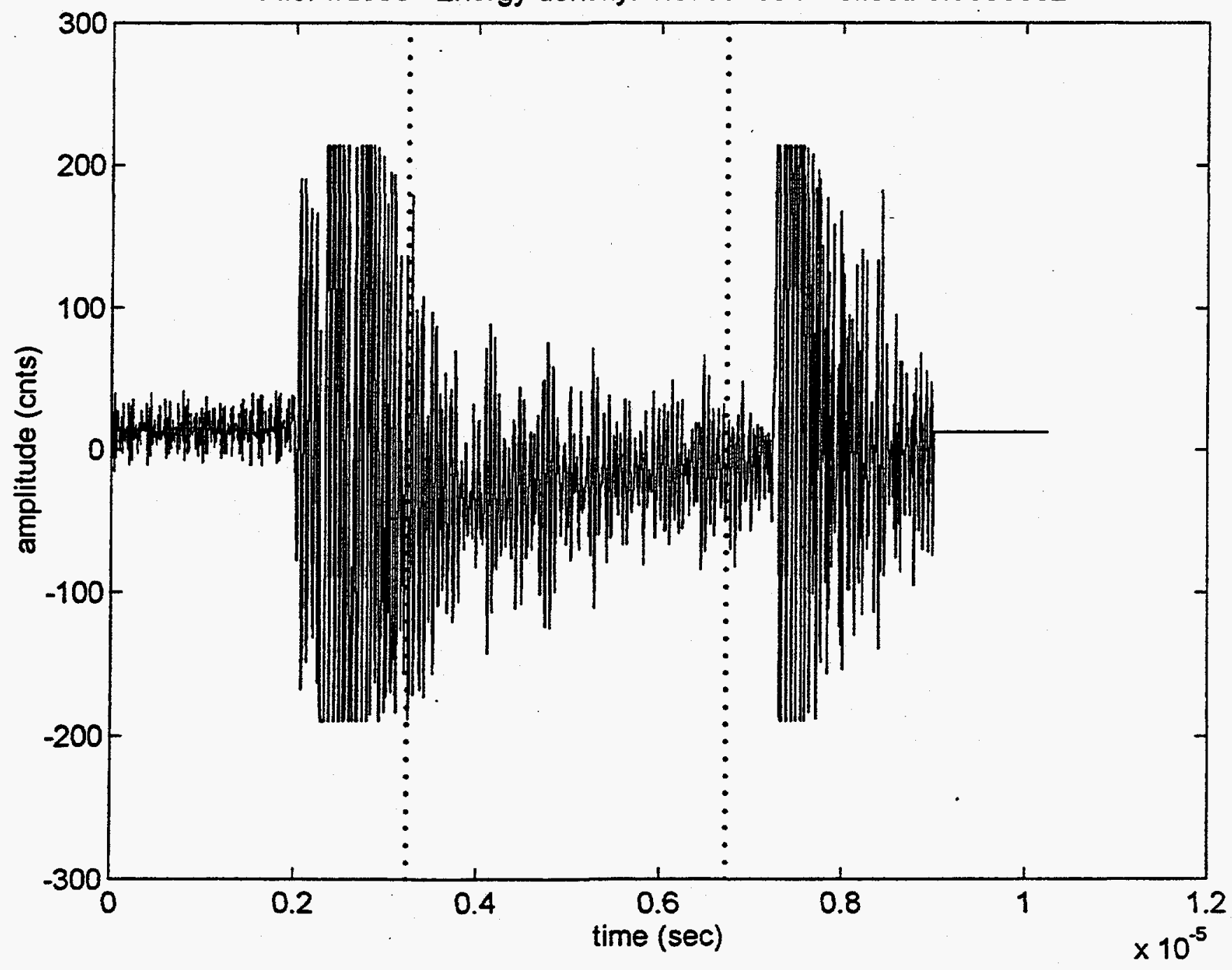


File: wd339 Energy density: $1.801 e+004$ offset: 0.00005176

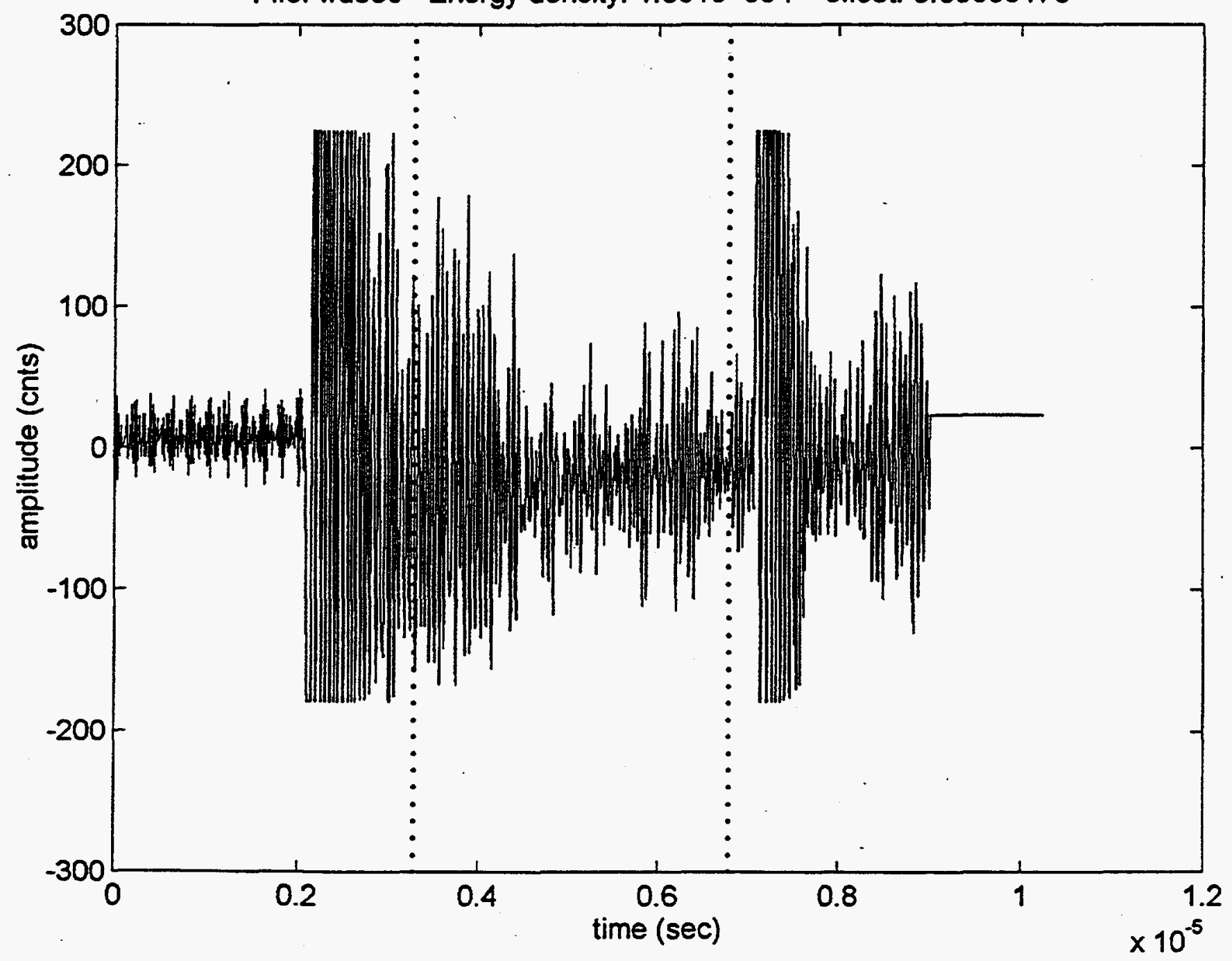




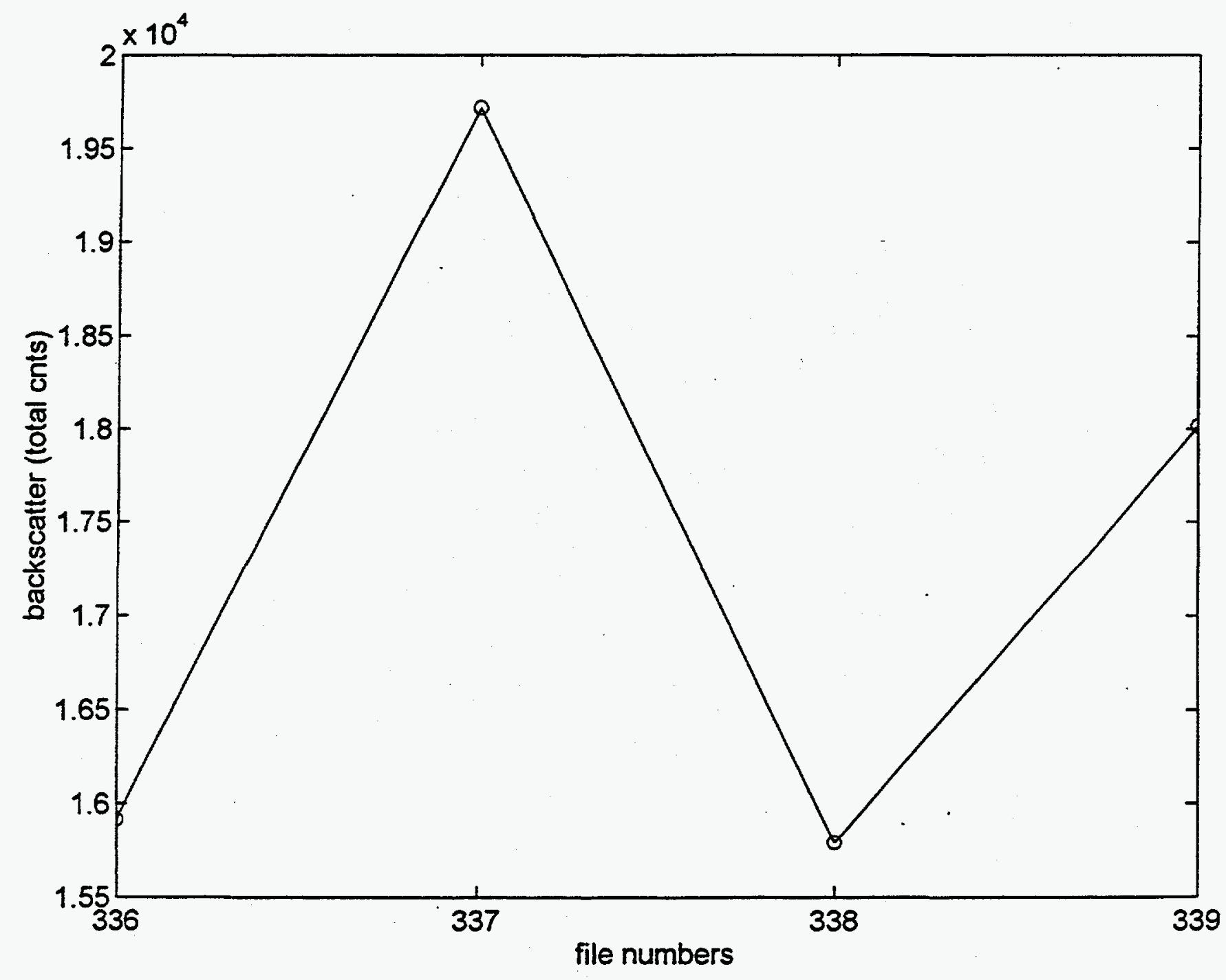


File: wd340 Energy density: $1.708 e+004$ offset: -0.00003125

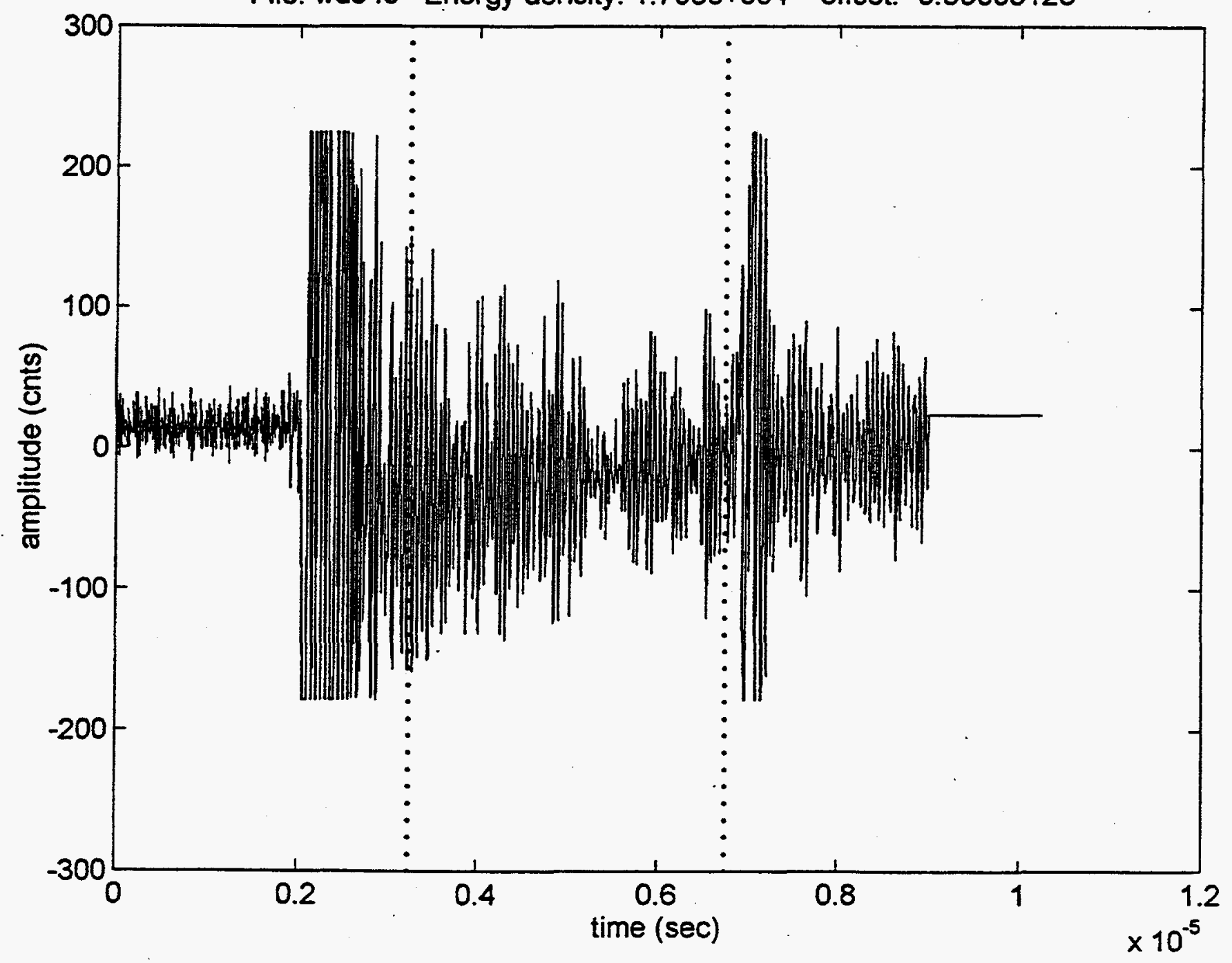


File: wd341 Energy density: $1.712 e+004$ offset: 0.00001562

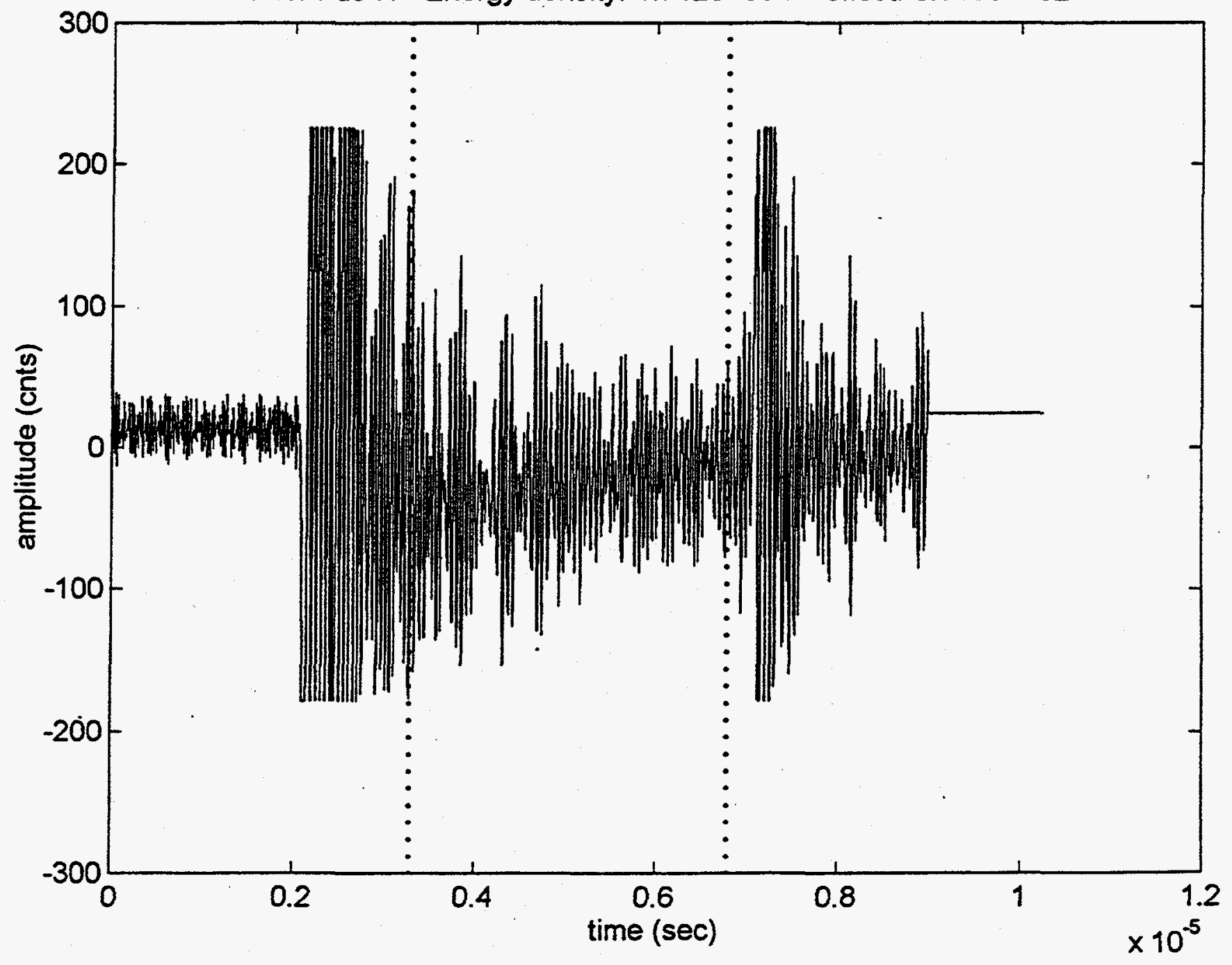


File: wd342 Energy density: $1.618 e+004$ offset: -0.00002051

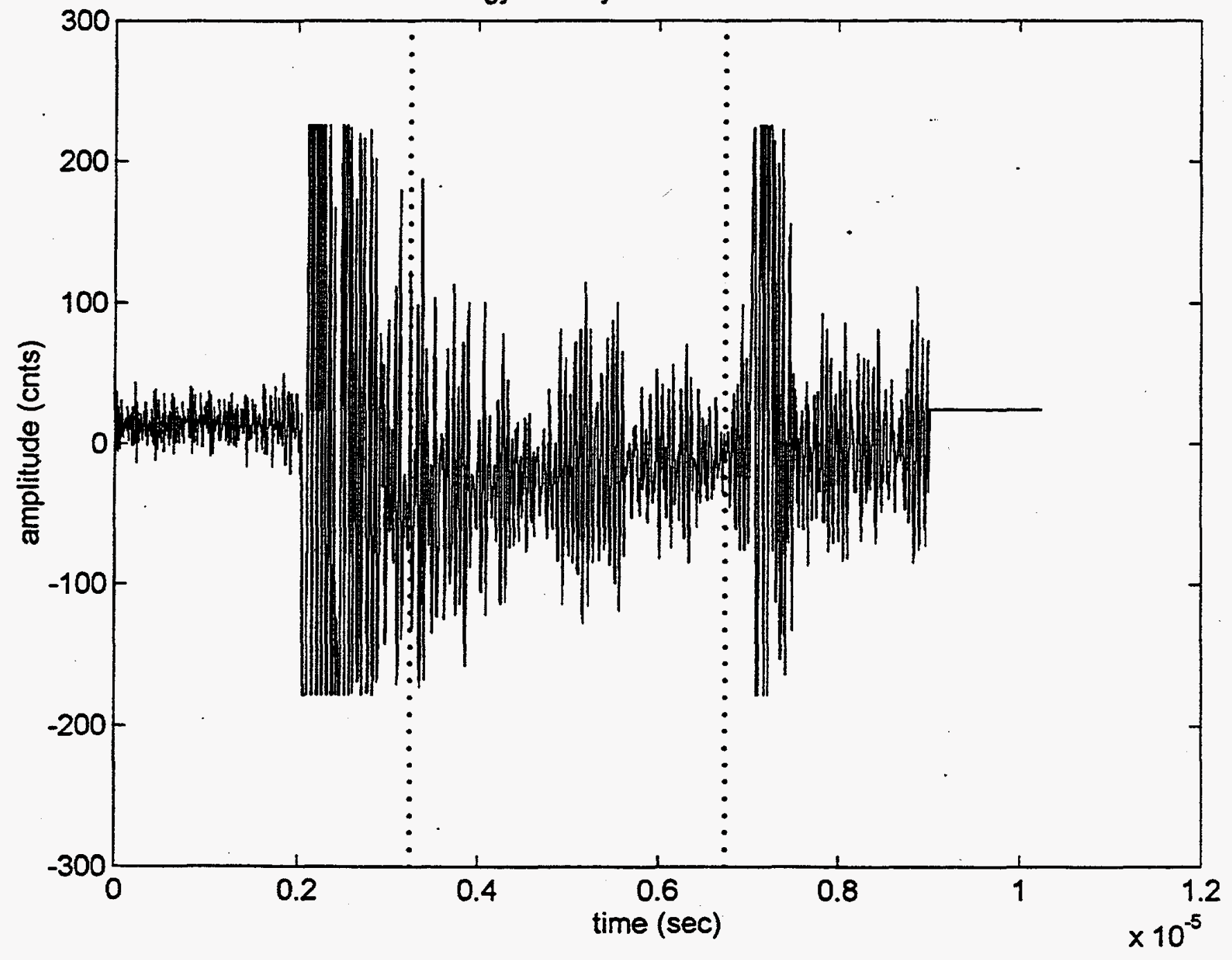


File: wd343 Energy density: $1.756 e+004$ offset: -0.00003516

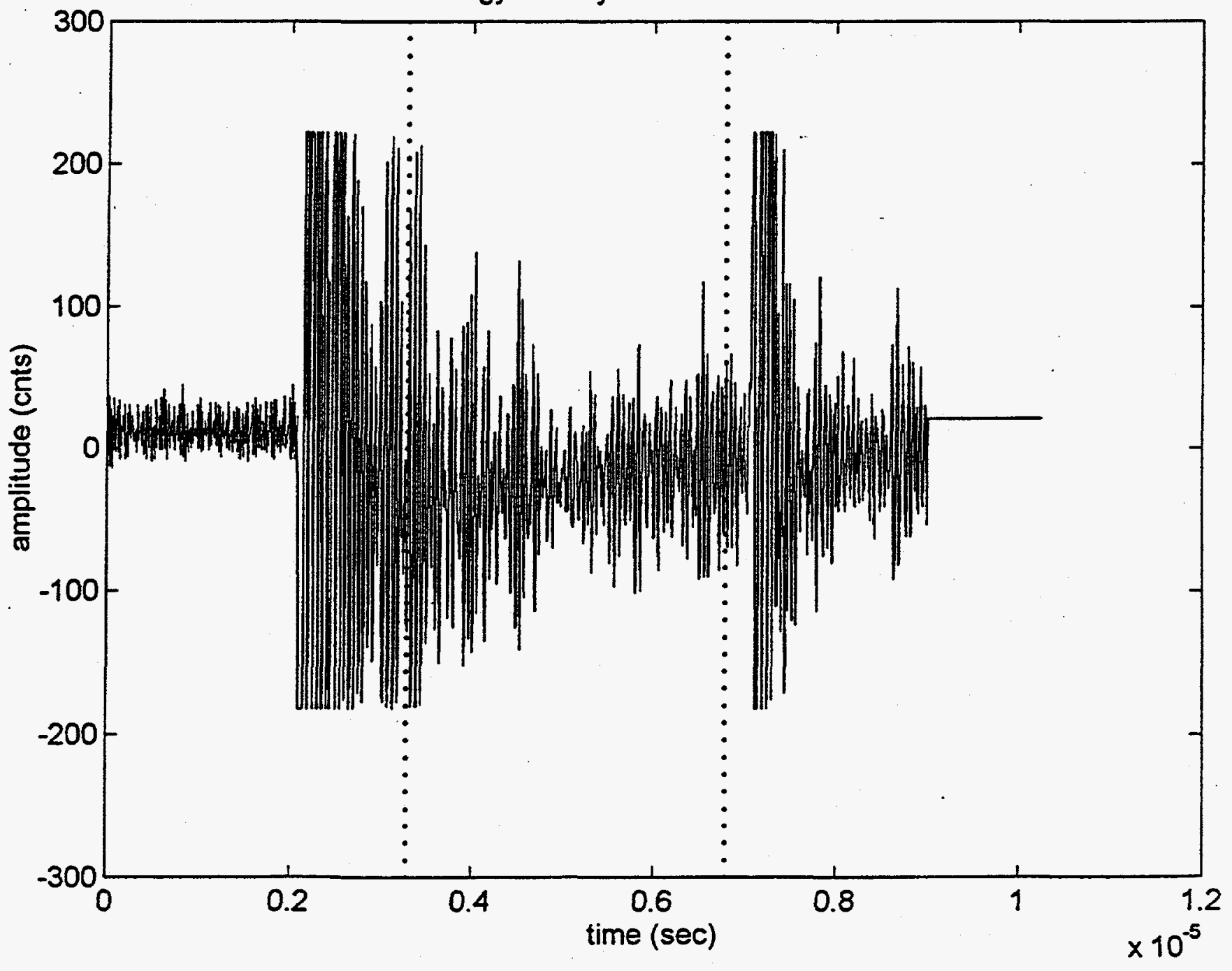


File: wd344 Energy density: $1.555 e+004$ offset: 0.0000166

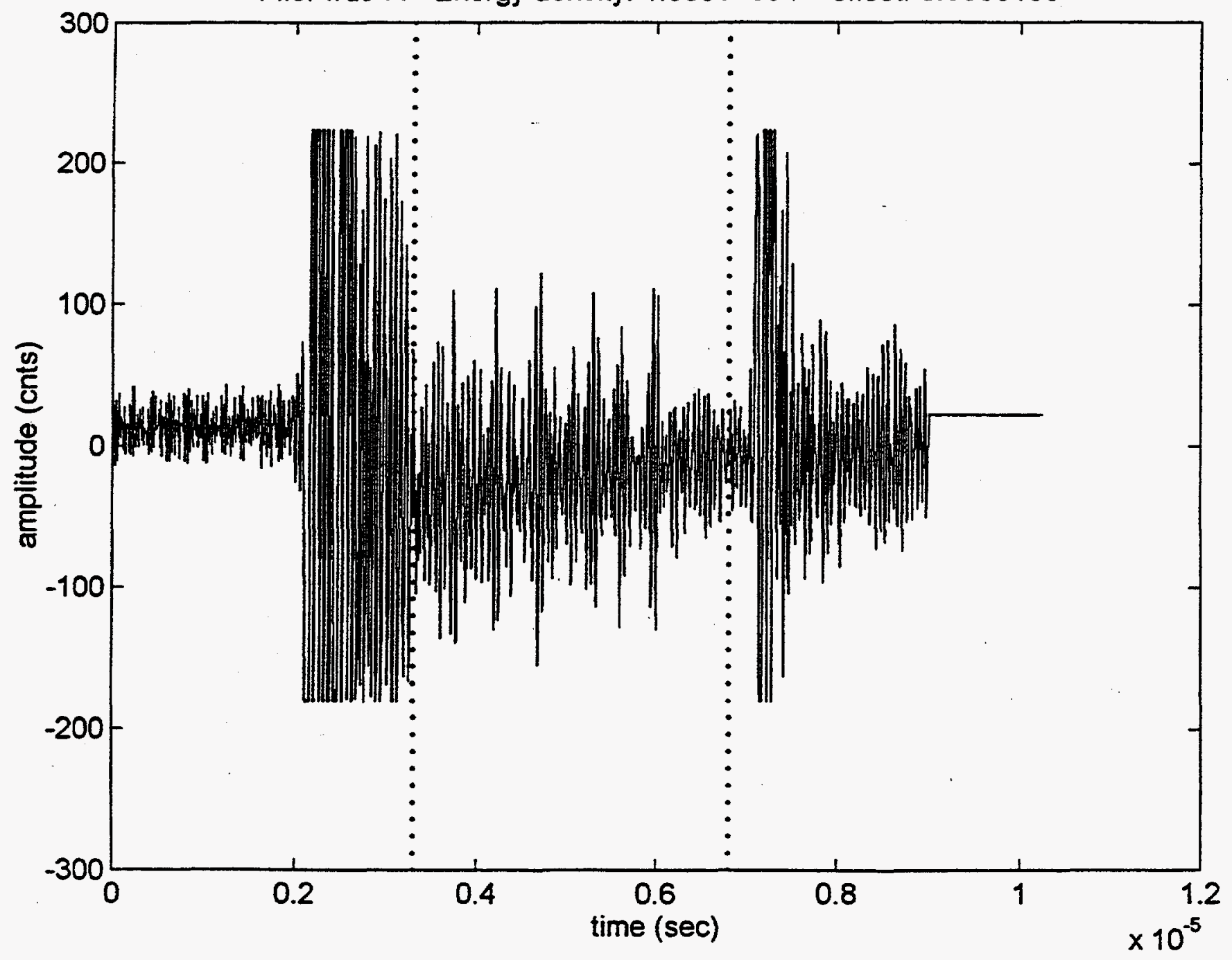




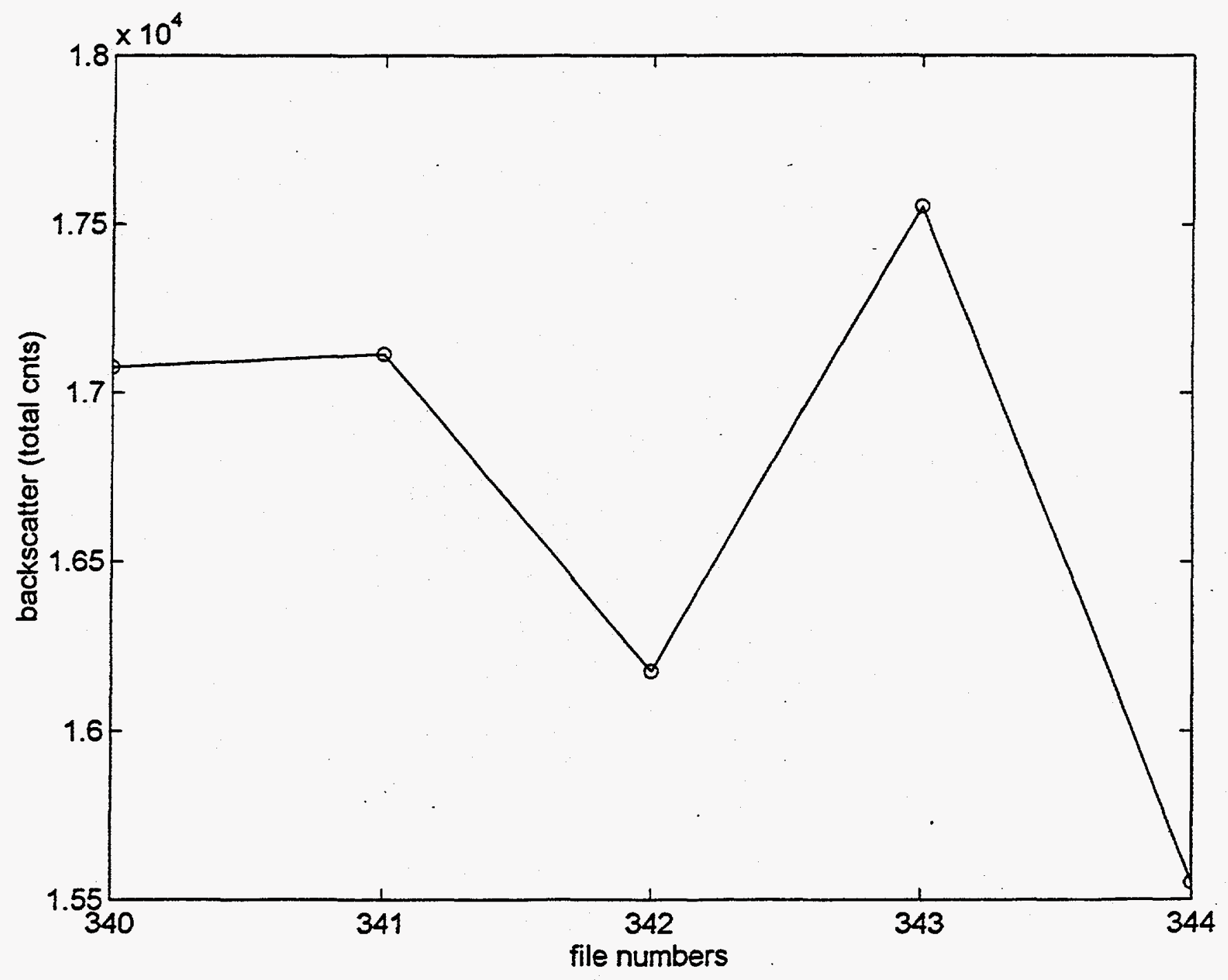


File: wd345 Energy density: $2.599 e+004$ offset: -0.00002734

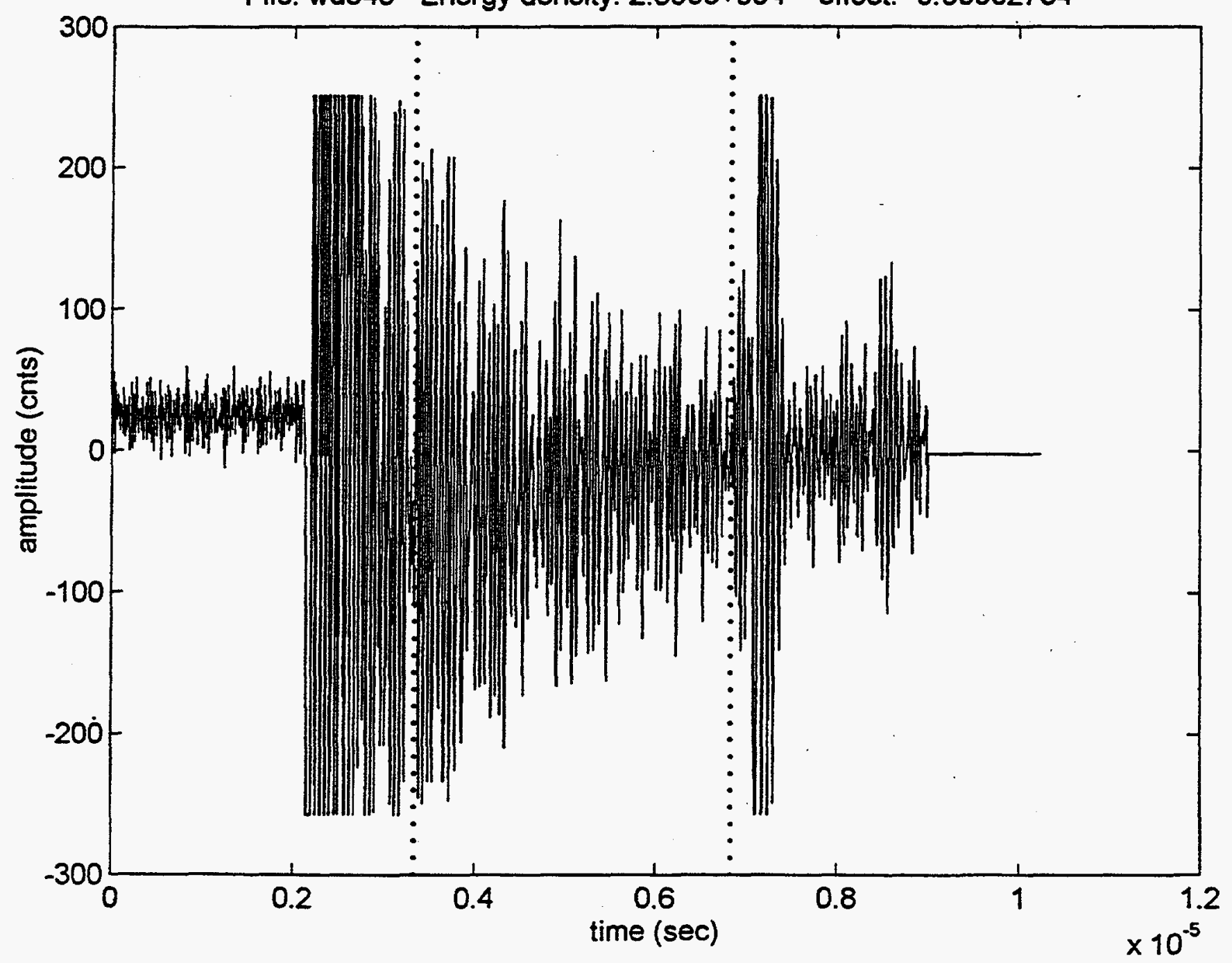


File: wd346 Energy density: $1.874 e+004$ offset: -0.0000166

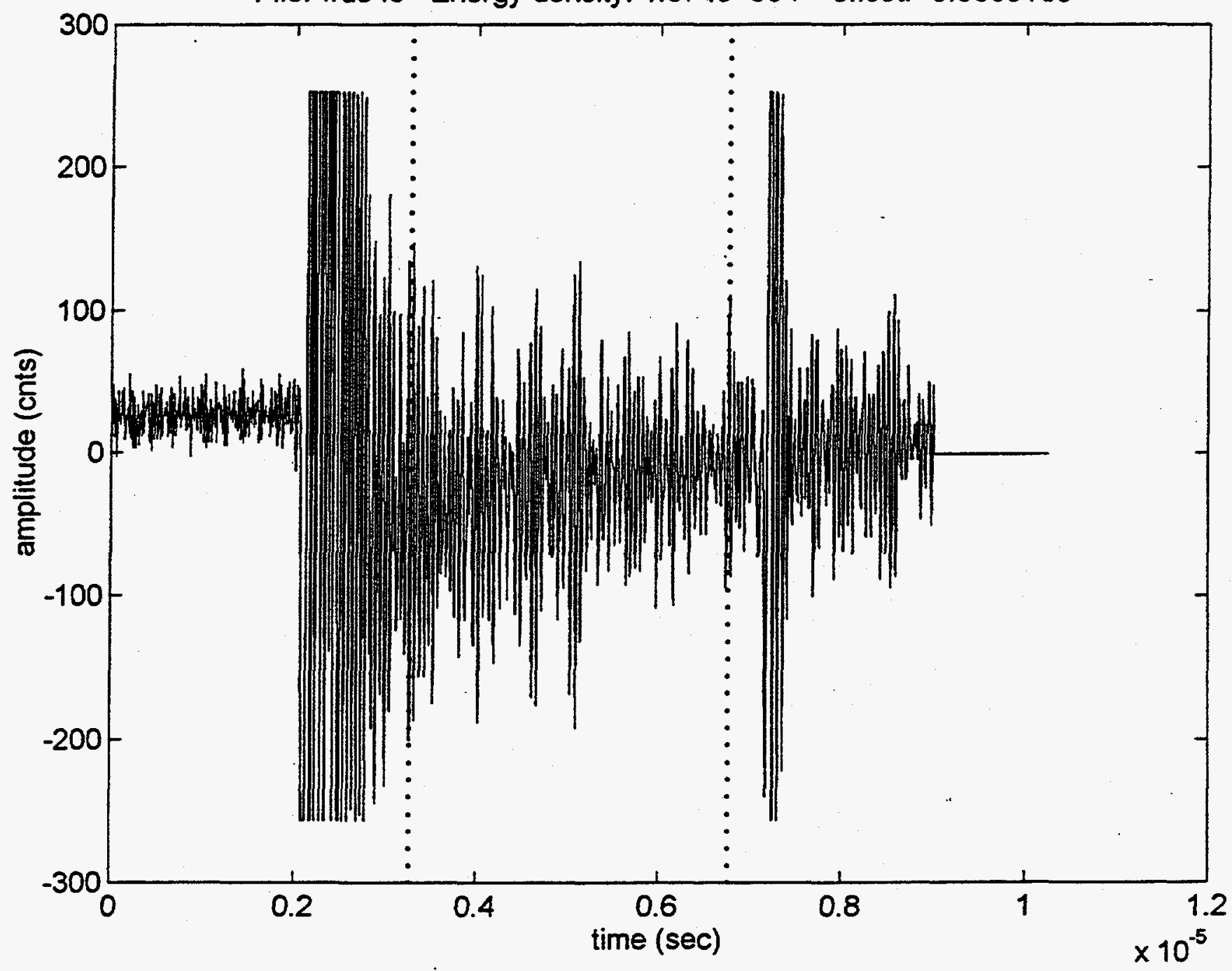


File: wd347 Energy density: $2.175 e+004$ offset: -0.00001465

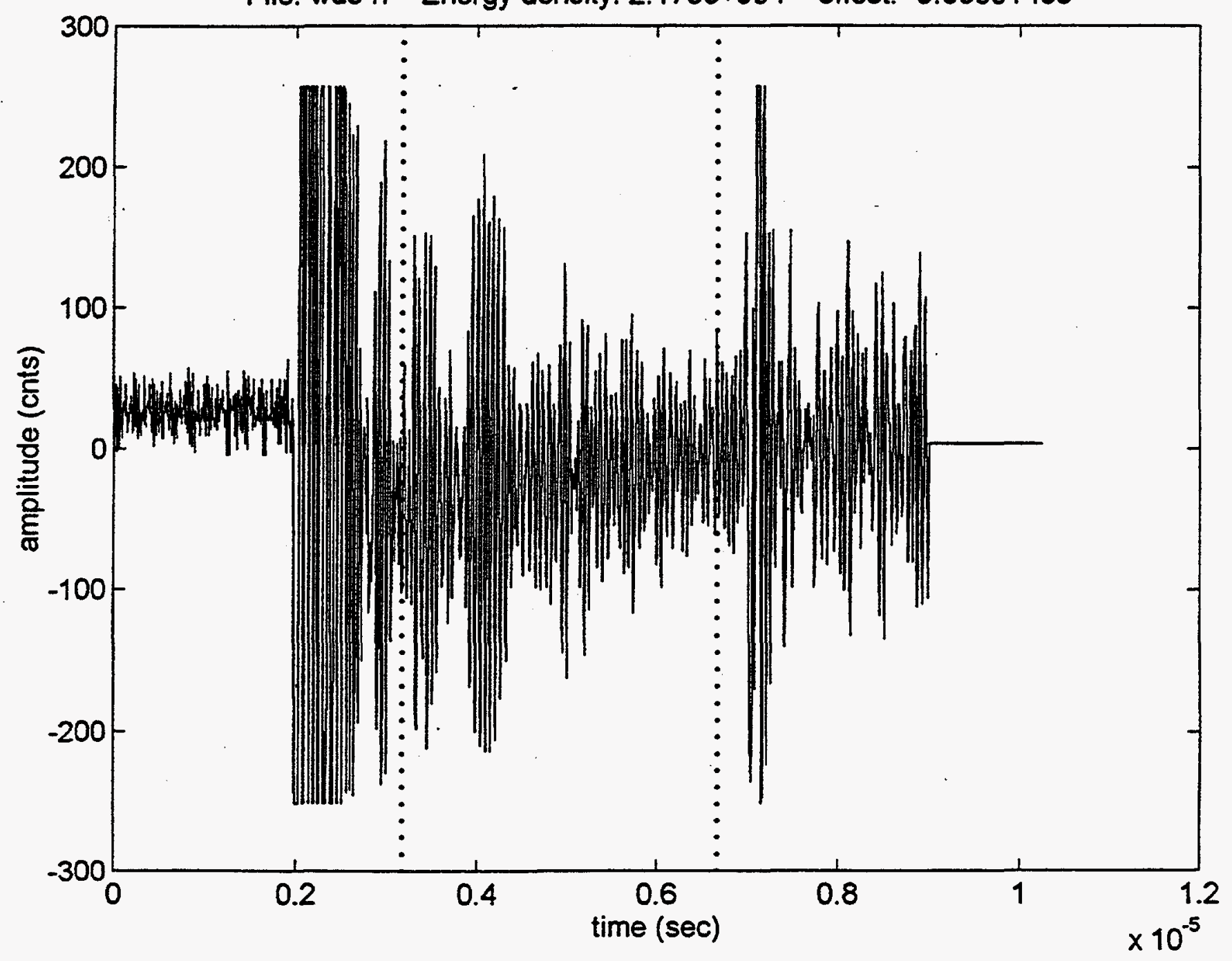


File: wd348 Energy density: $1.998 e+004$ offset: 0.00004395

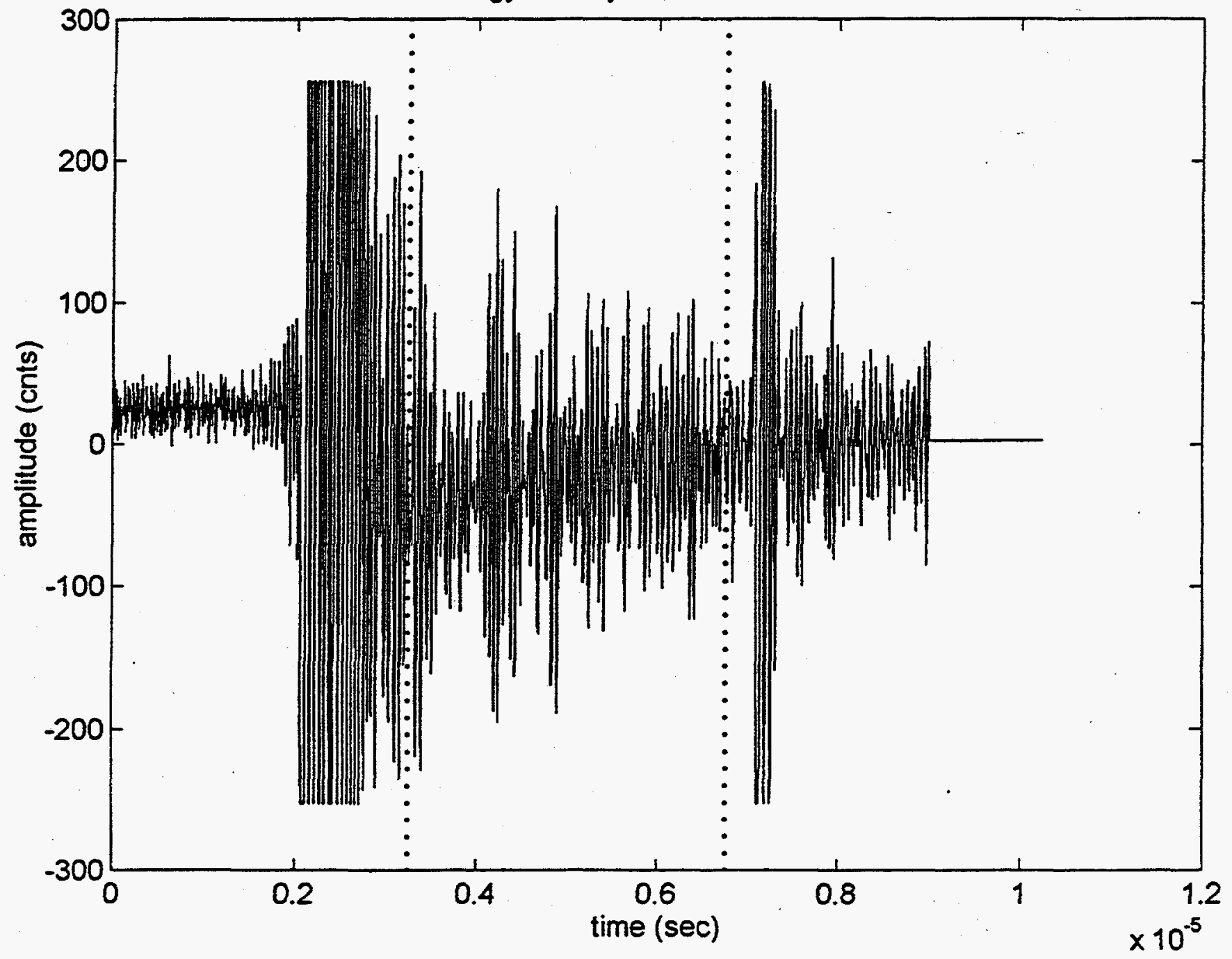


File: wd349 Energy density: $2.356 e+004$ offset: -0.0000293

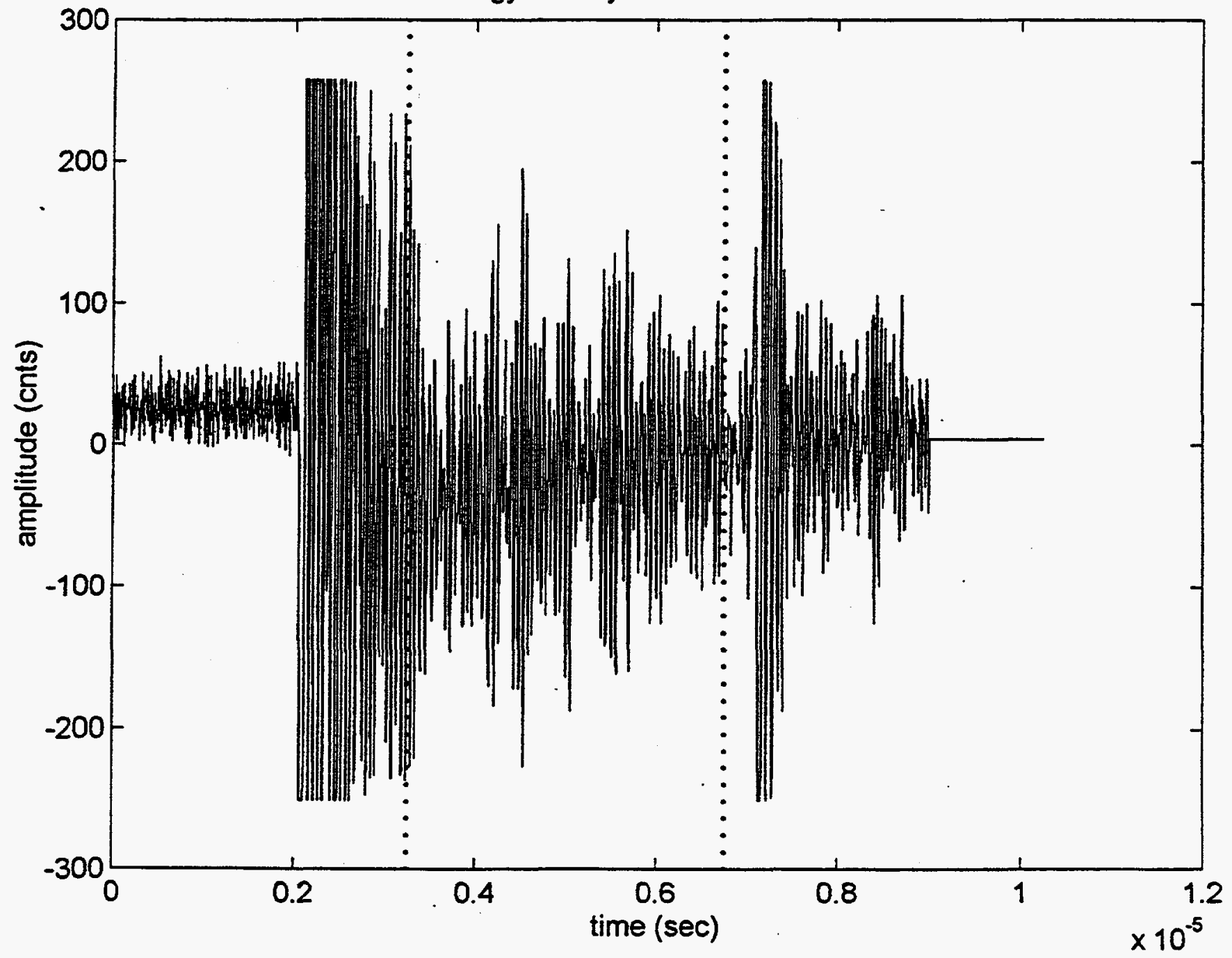




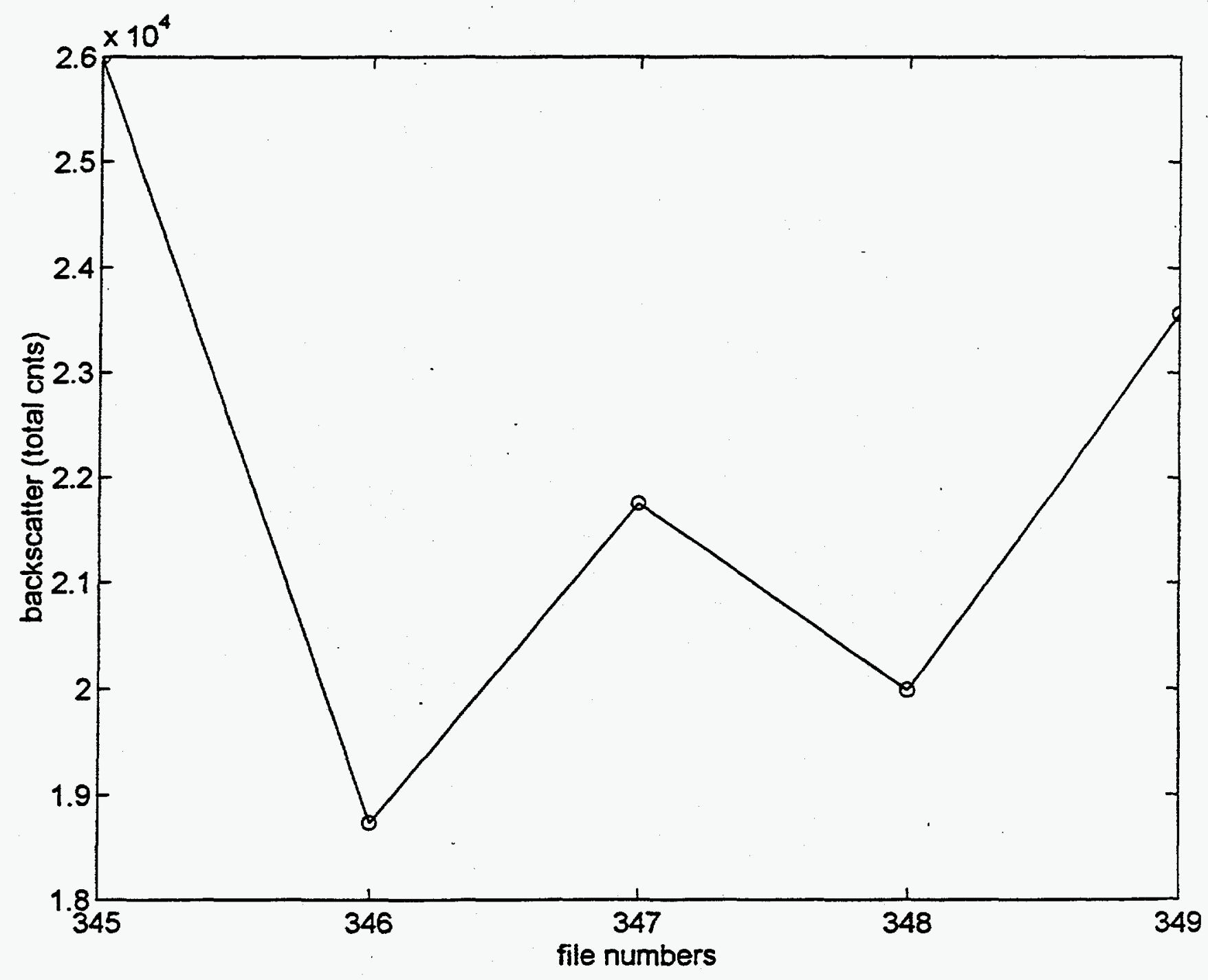


File: wd350 Energy density: $1.878 e+004$ offset: $3.906 e-006$

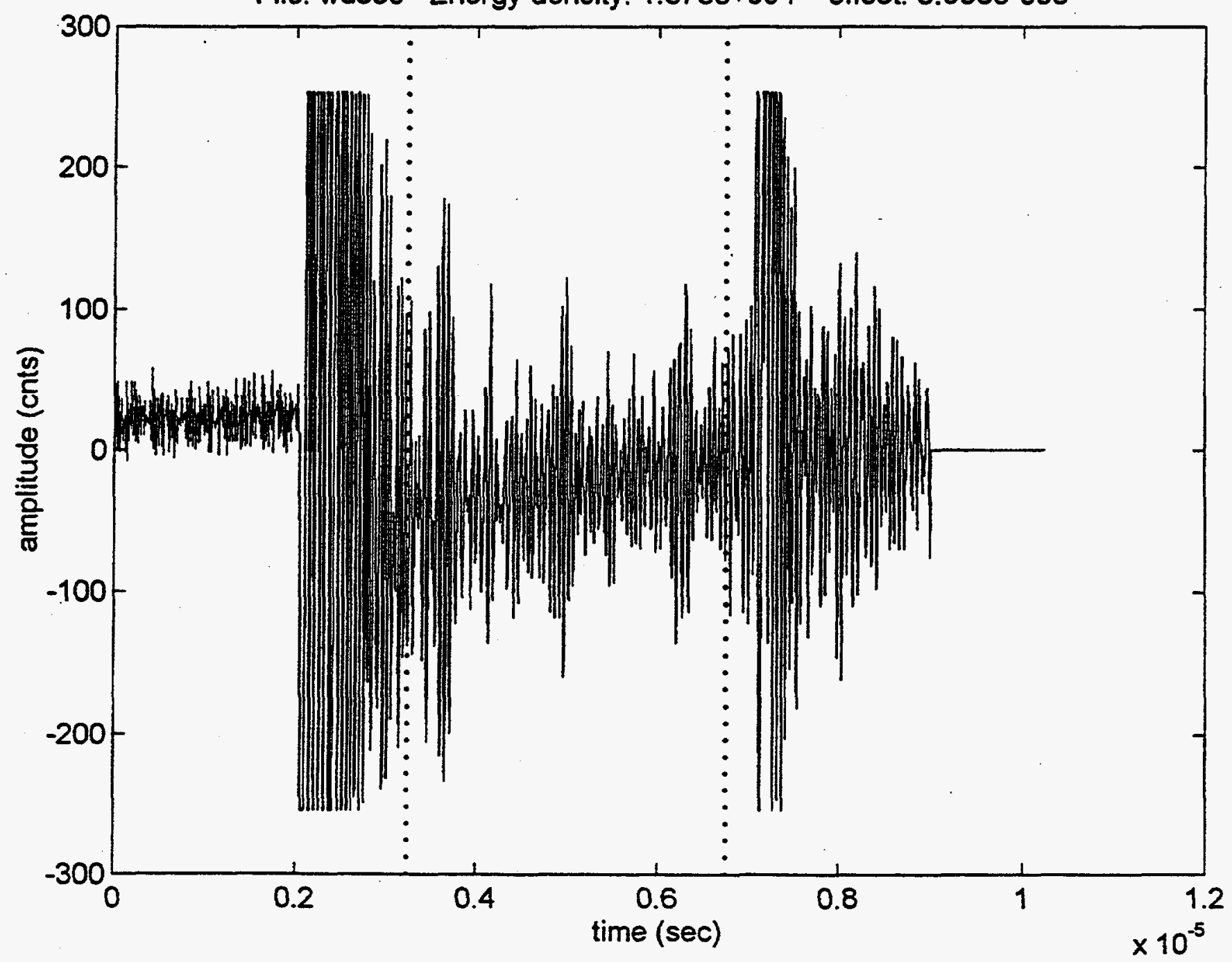


File: wd351 Energy density: $1.693 e+004$ offset: -0.00002832

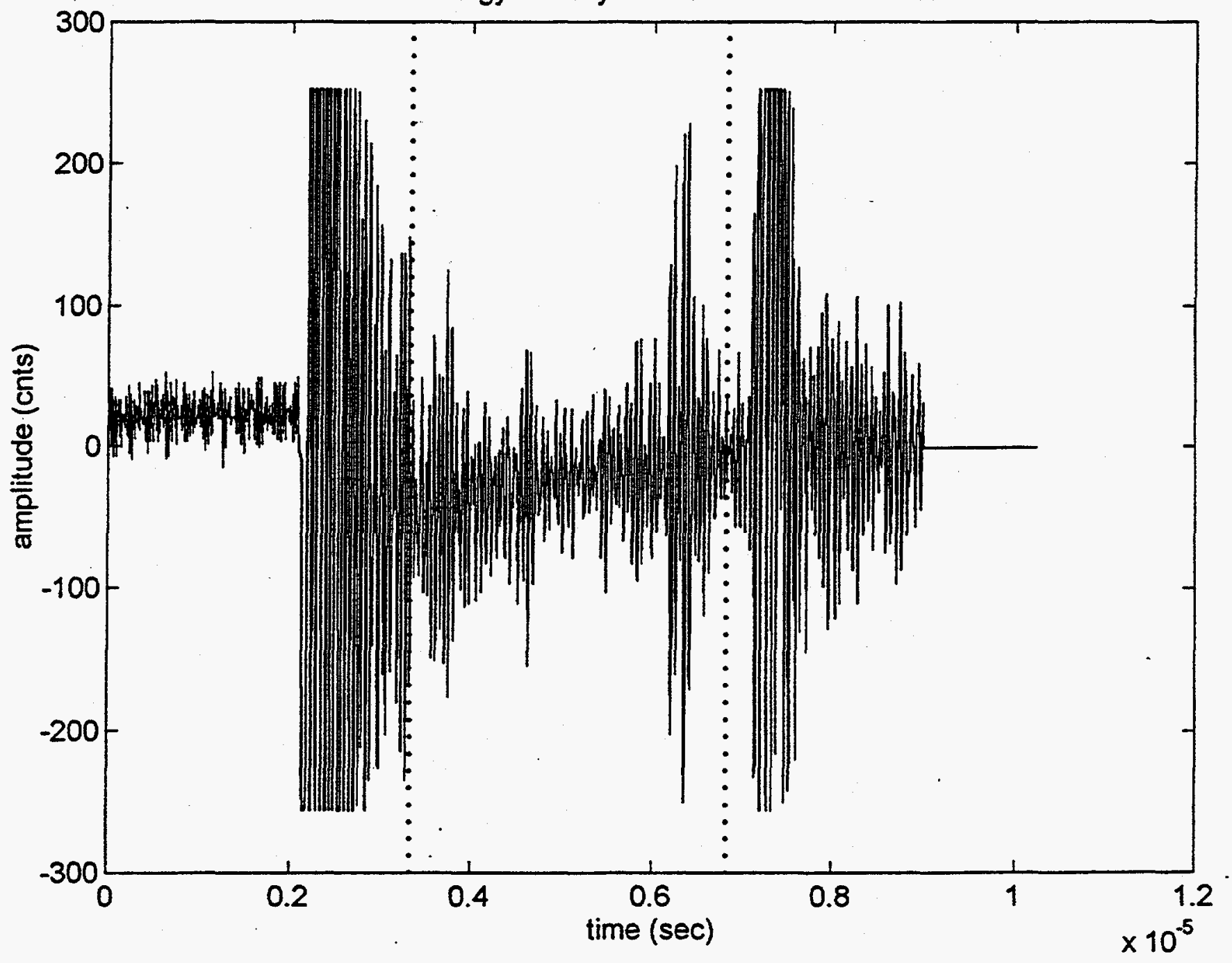


File: wd352 Energy density: $1.688 e+004$ offset: 0.0000166

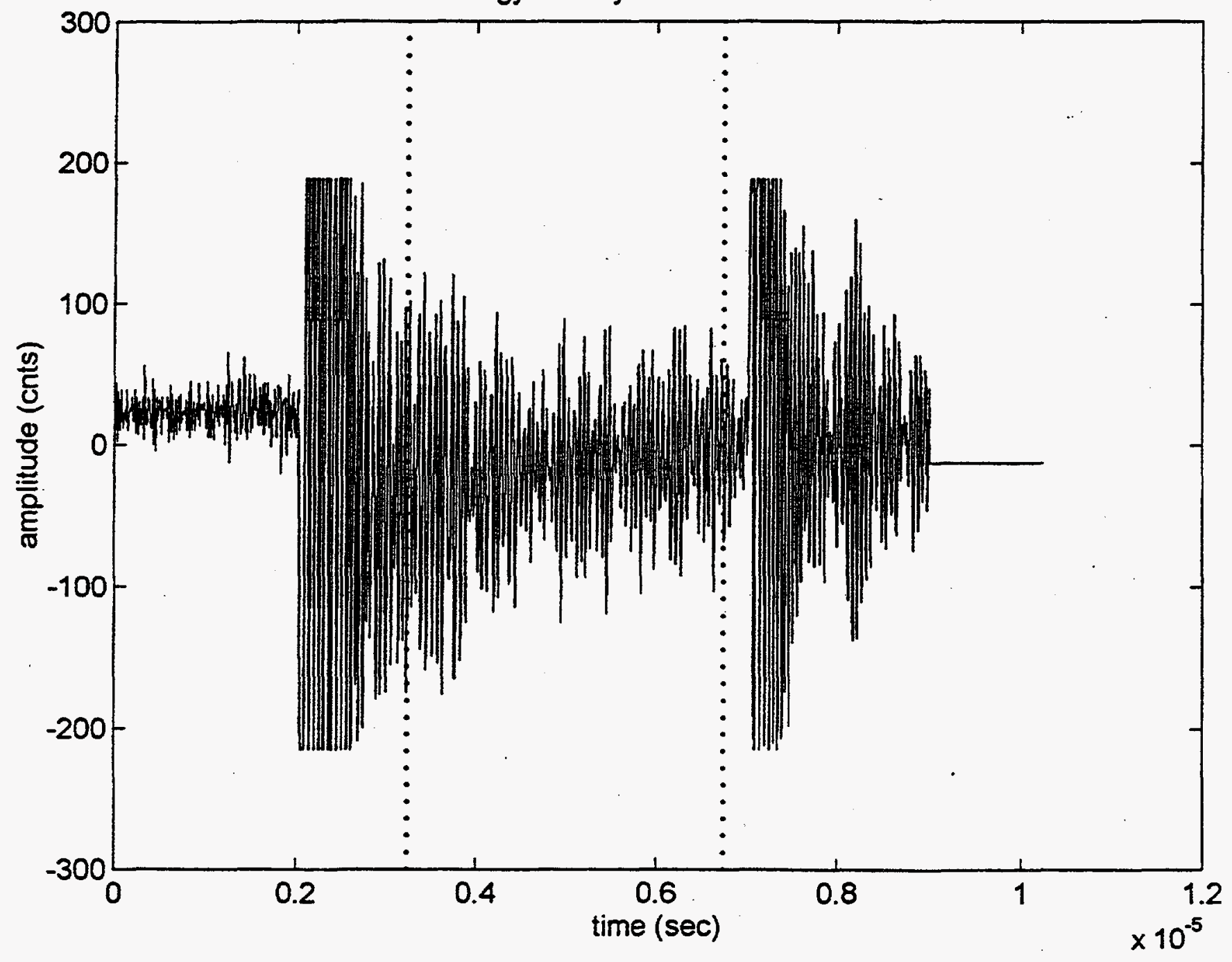


File: wd353 Energy density: $1.601 e+004$ offset: -0.00002539

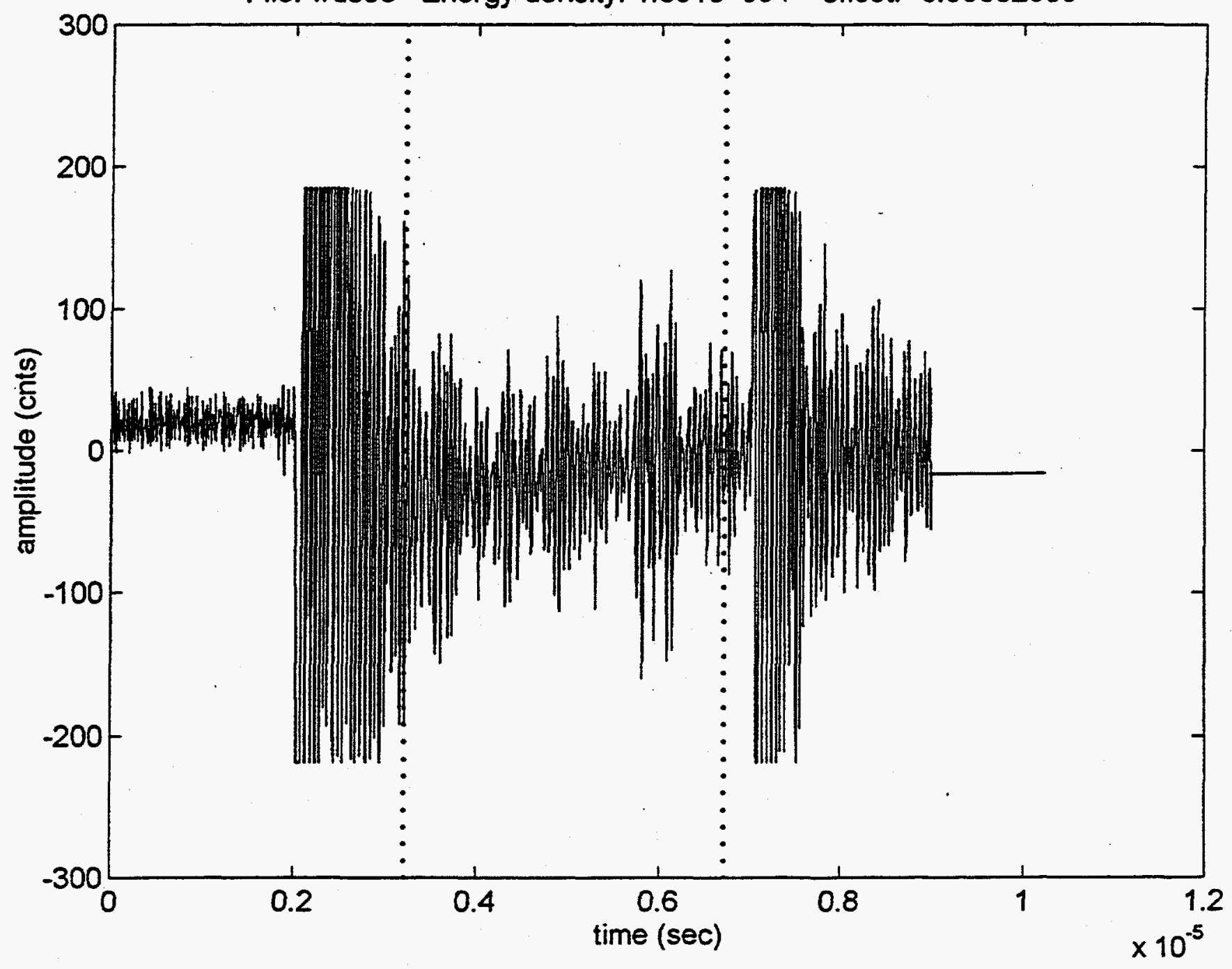


File: wd354 Energy density: 1.935 e+004 offset: 0.00001855

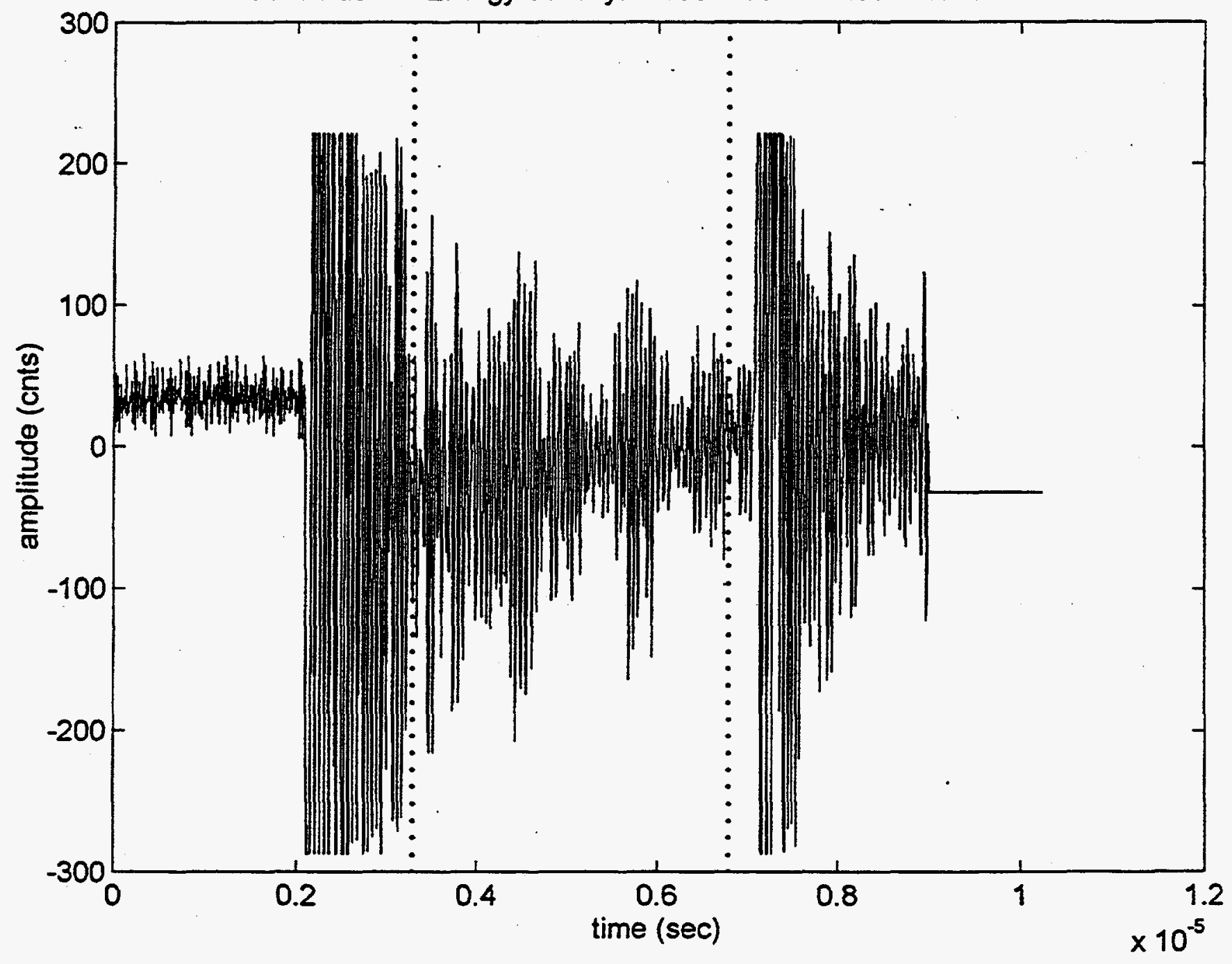




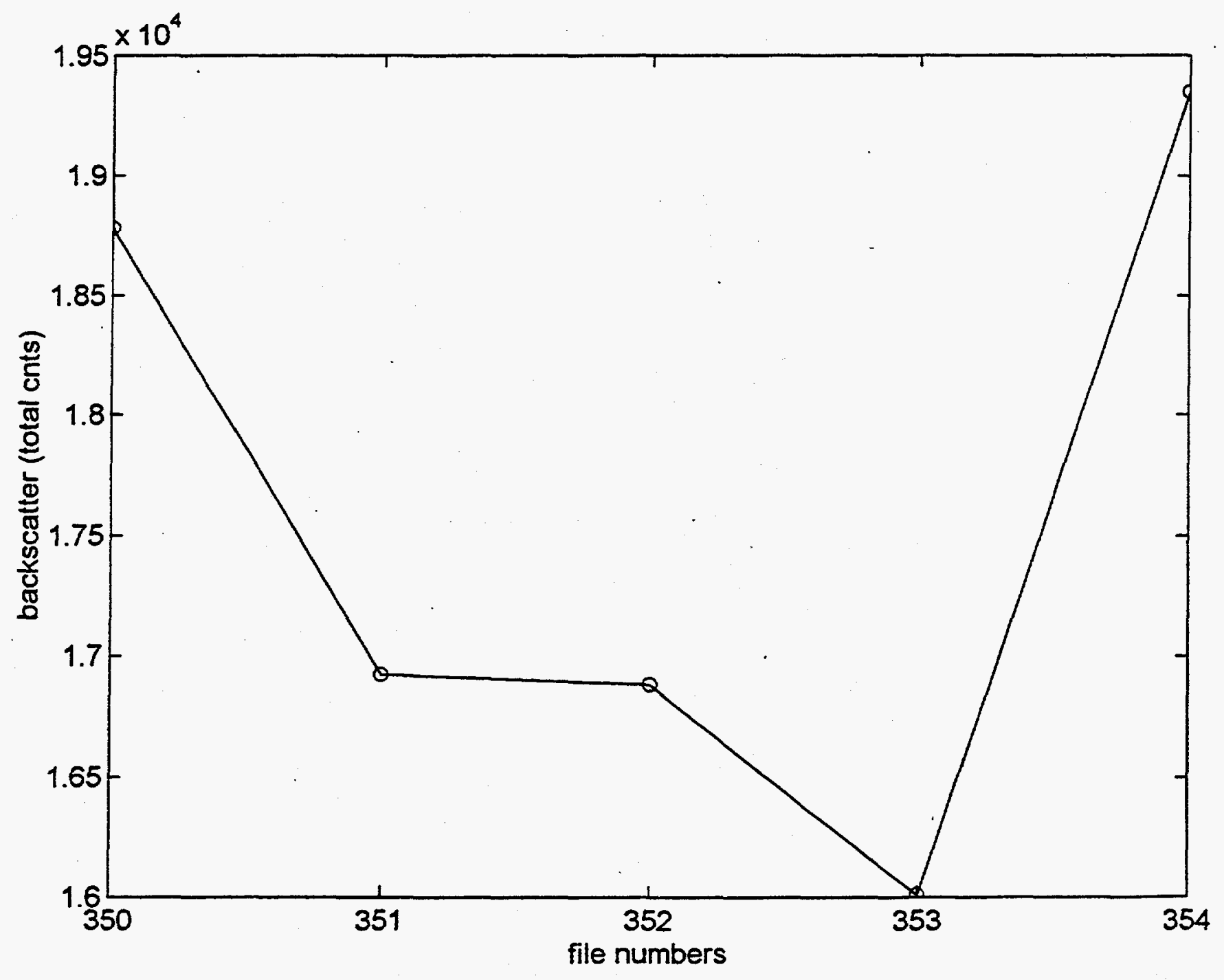


File: wd355 Energy density: $1.527 e+004$ offset: $-2.93 e-006$

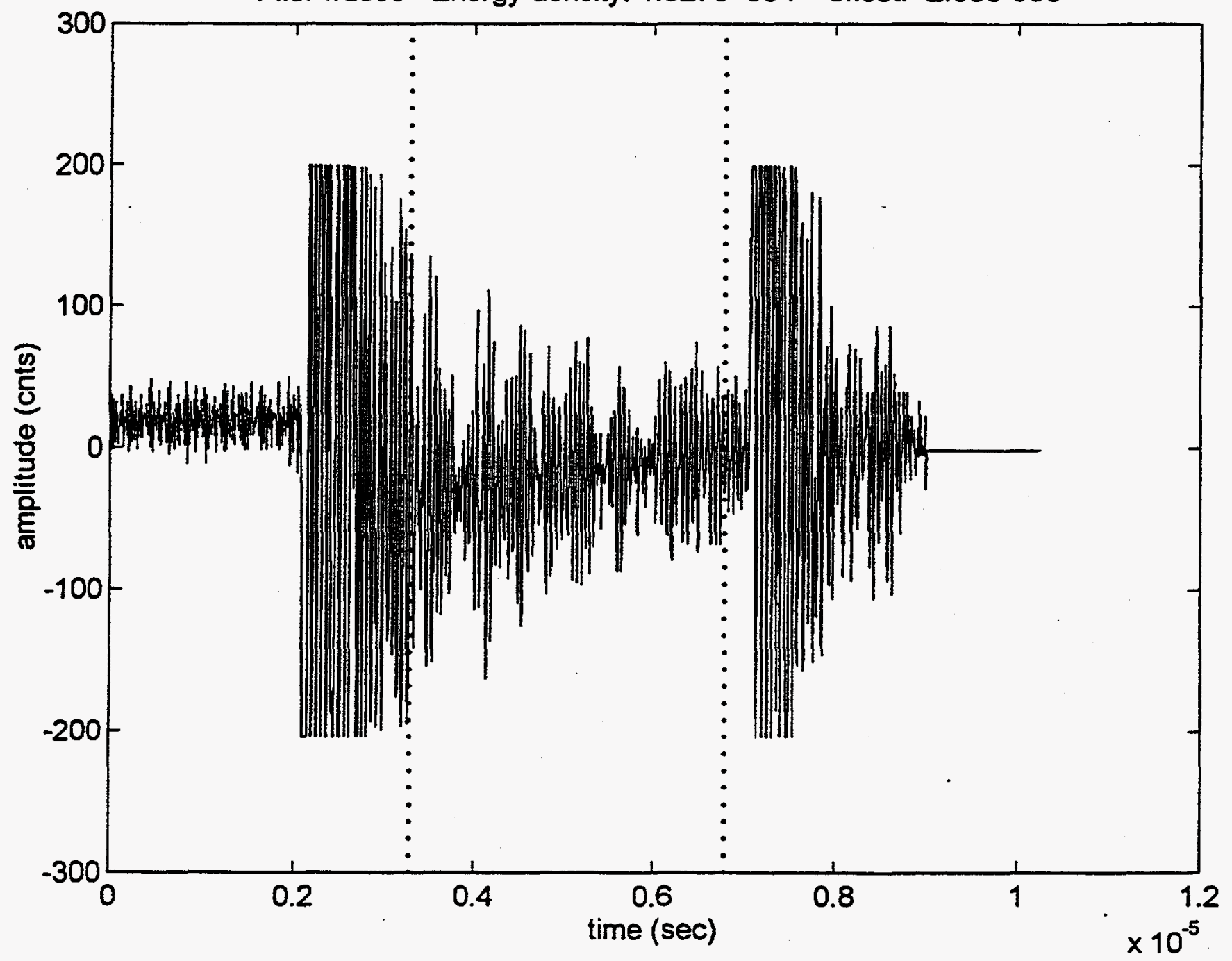


File: wd356 Energy density: $1.441 e+004$ offset: -0.00005566

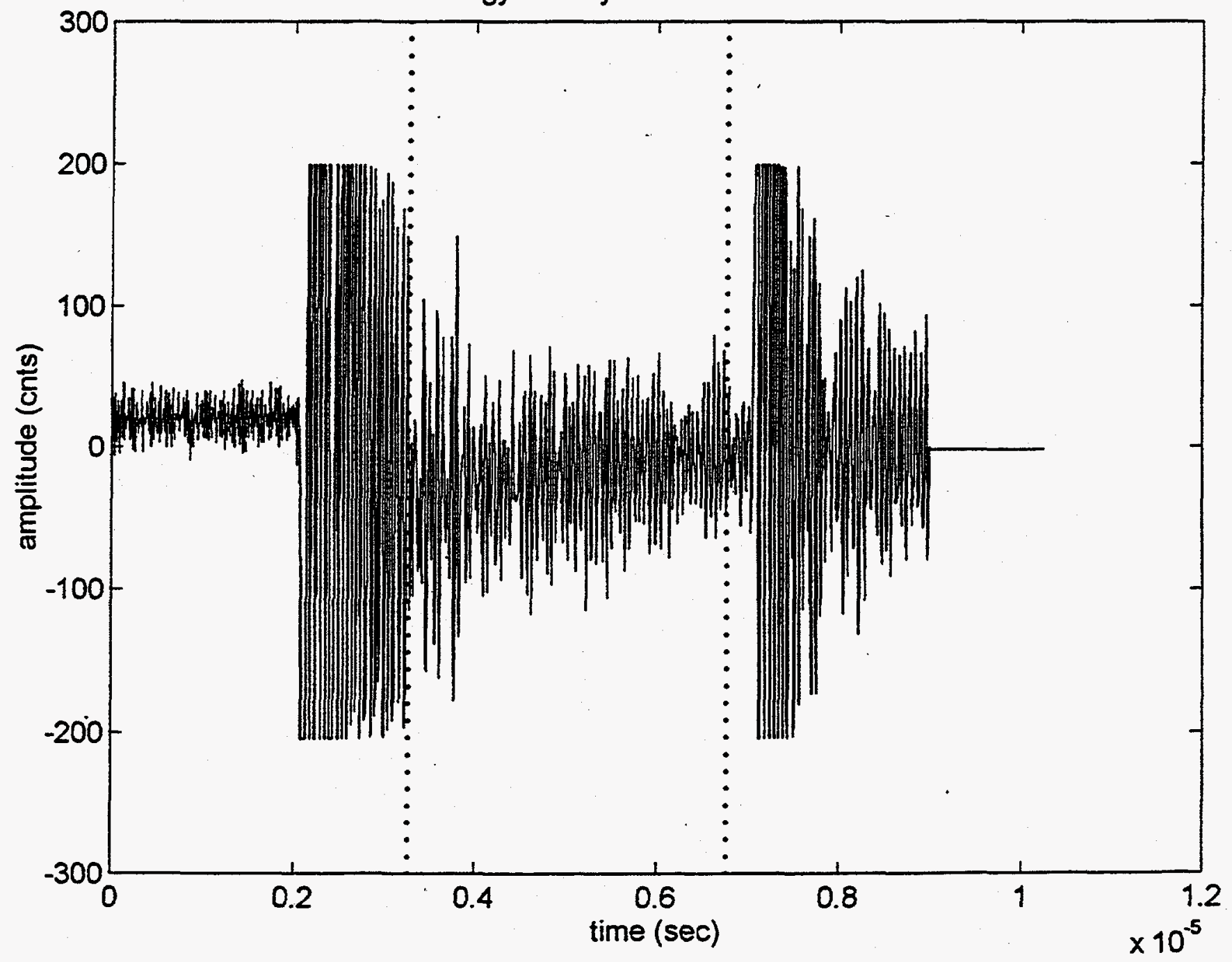


File: wd357 Energy density: $1.38 e+004$ offset: 0.0000166

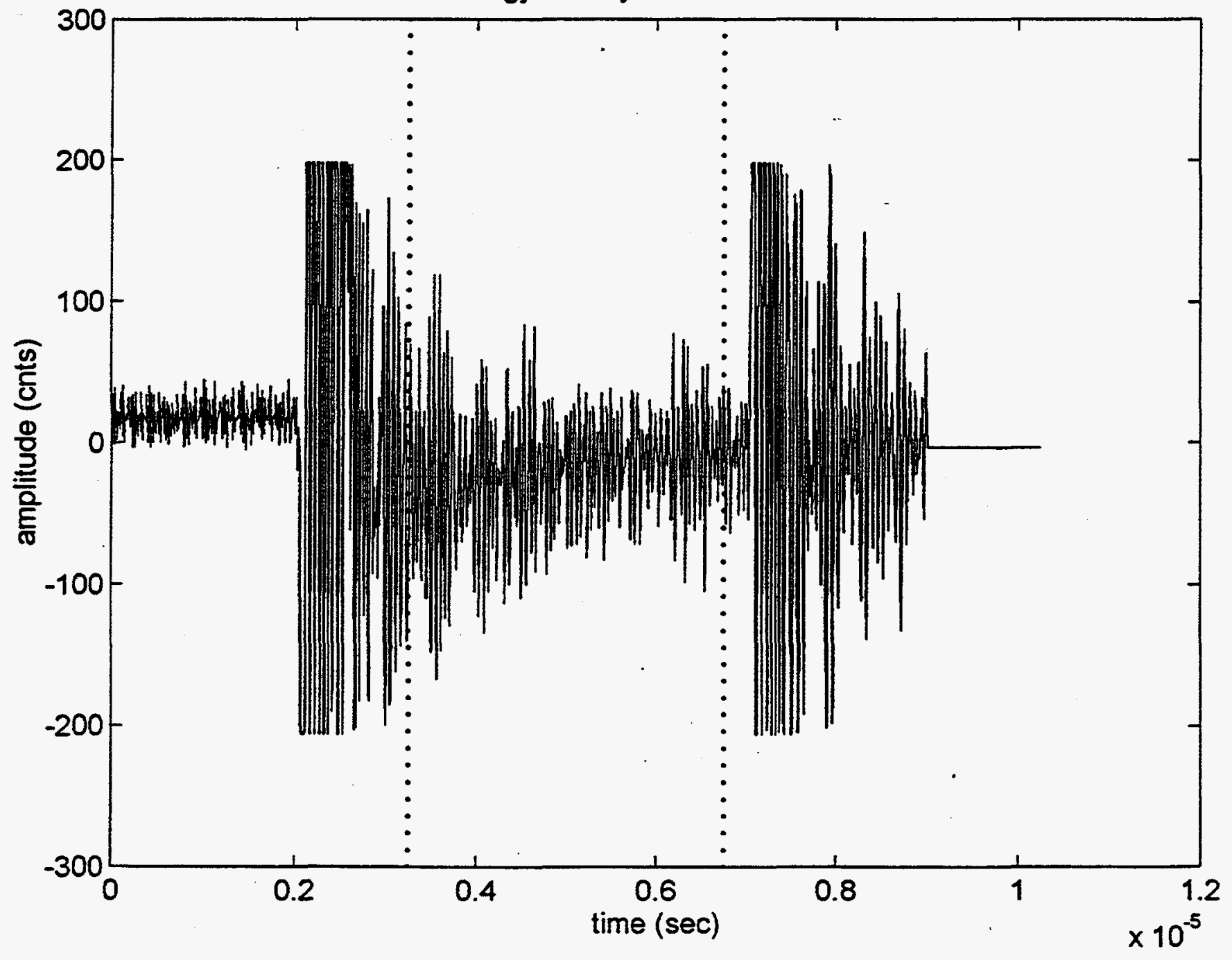


File: wd358 Energy density: $1.54 e+004$ offset: 0.00002734

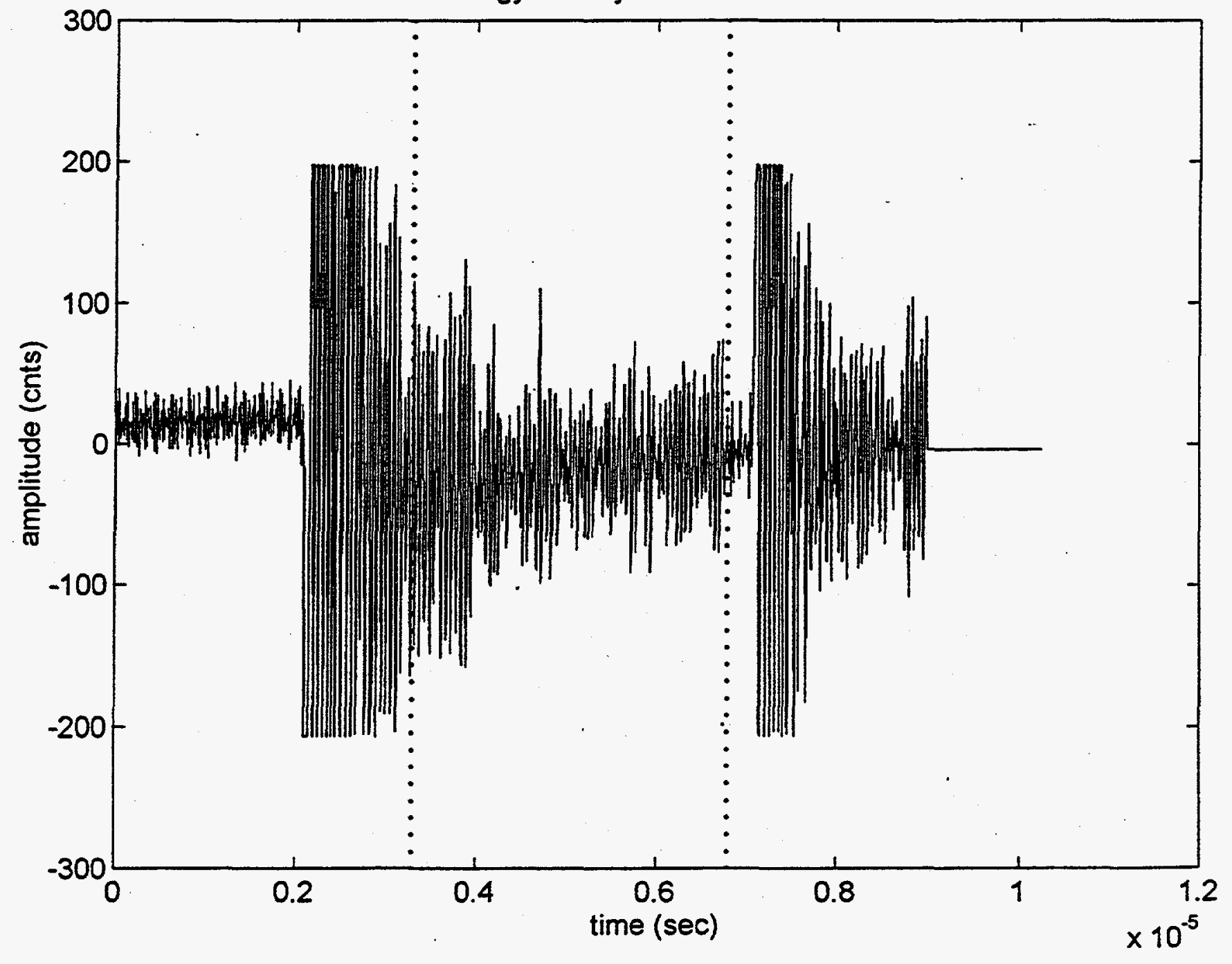


File: wd359 Energy density: $1.385 e+004$ offset: $9.766 e-007$

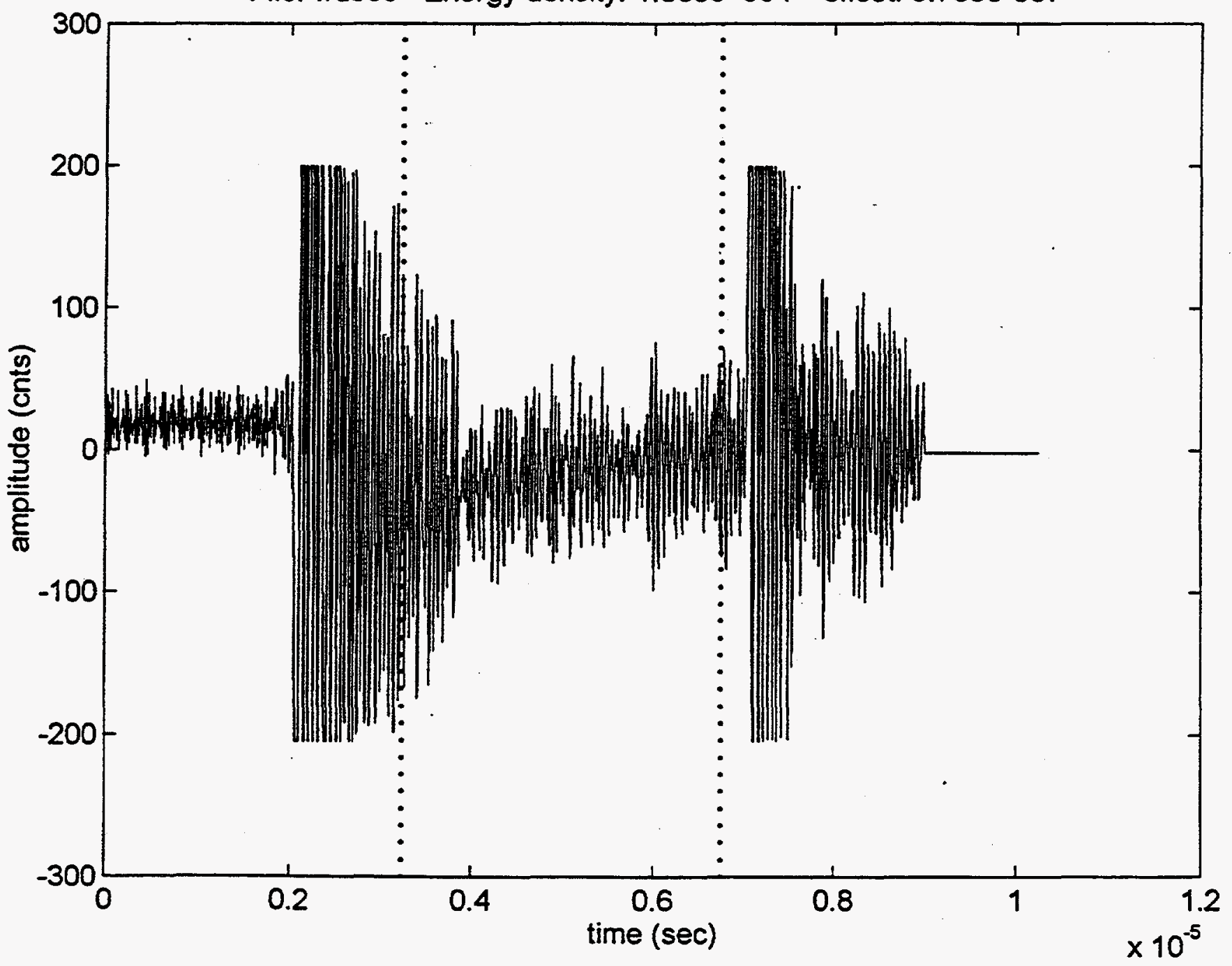




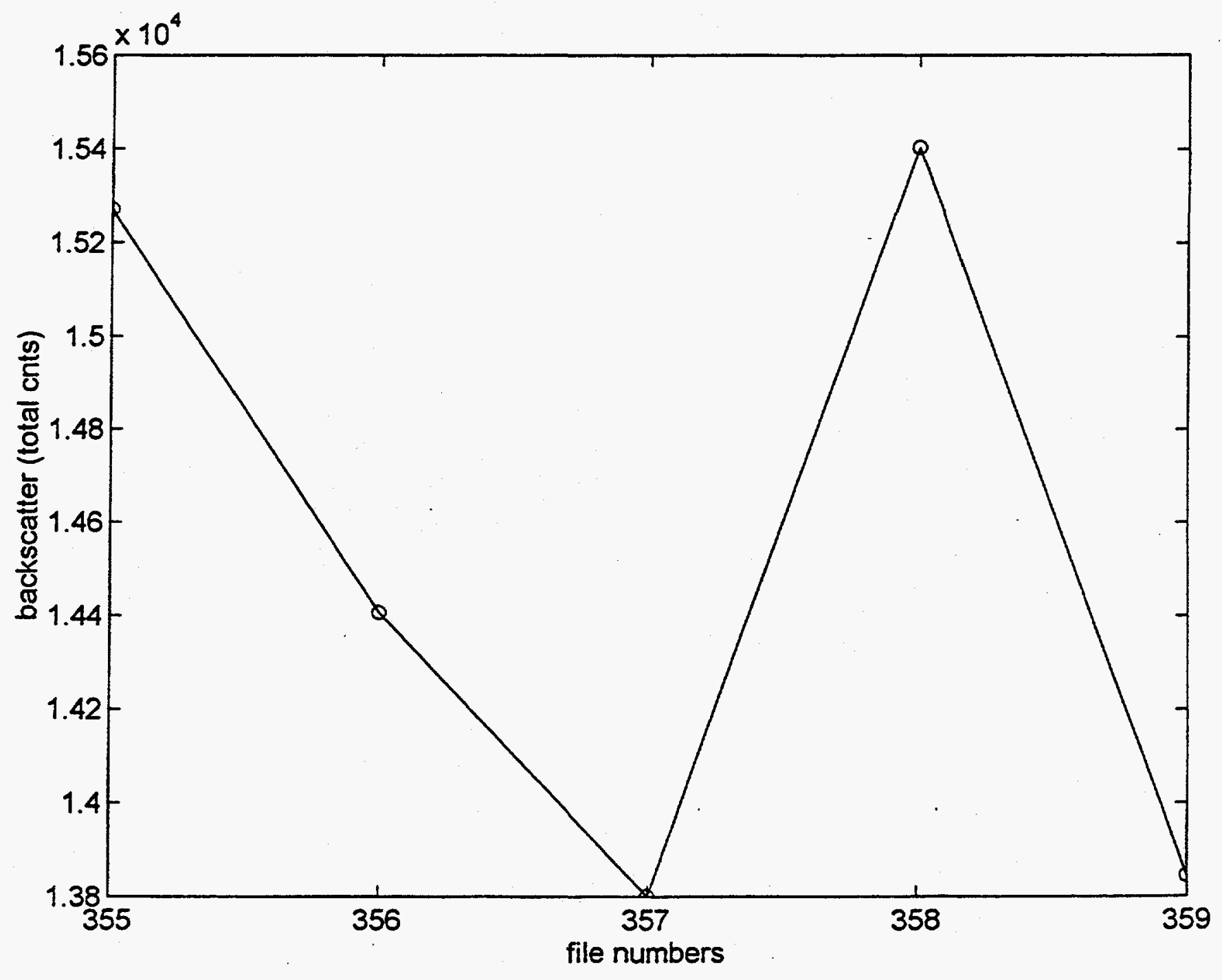


File: wd360 Energy density: $1.445 e+004$ offset: -0.00003125

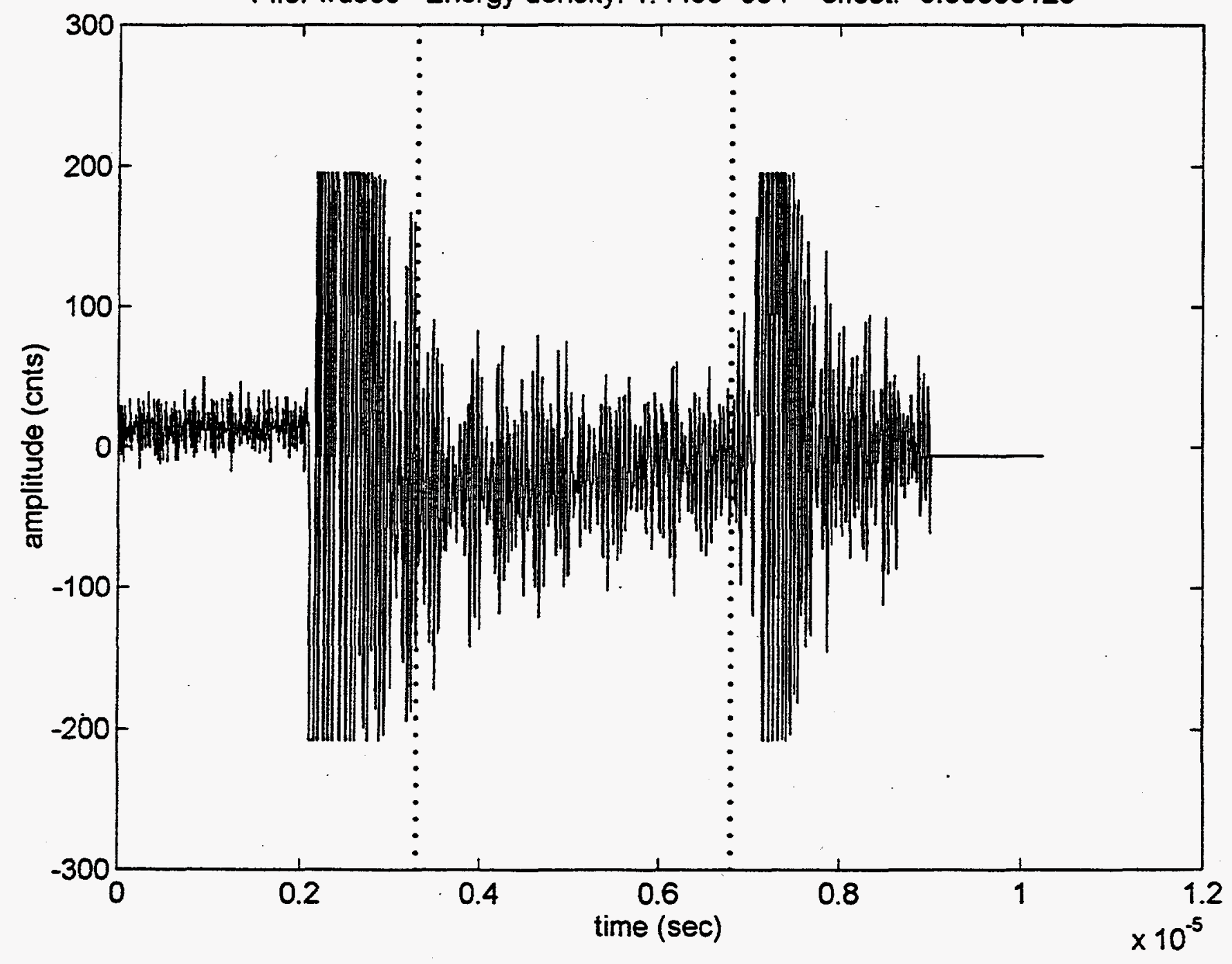


File: wd361 Energy density: $1.562 e+004$ offset: $9.766 e-007$

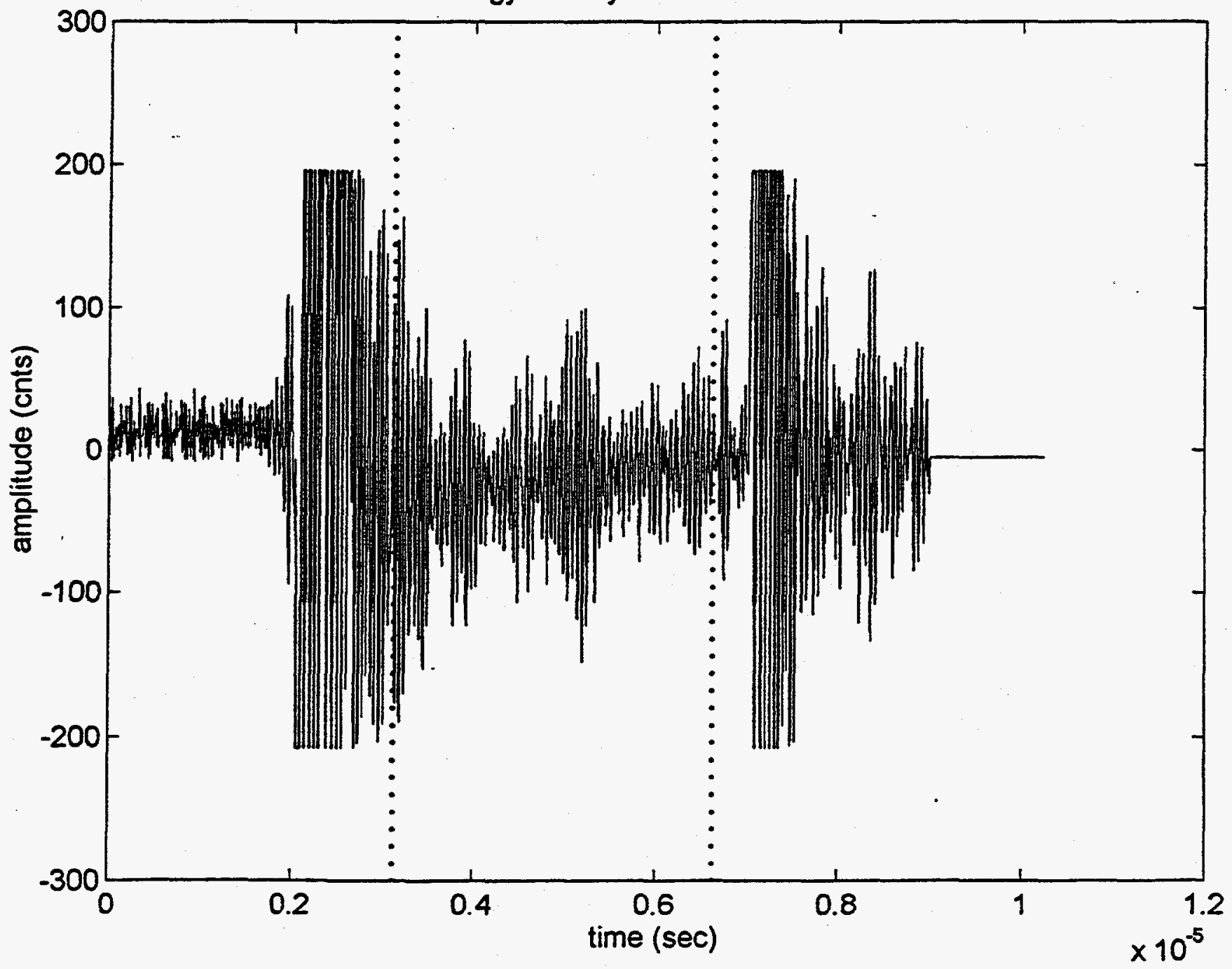


File: wd362 Energy density: $1.608 \mathrm{e}+004$ offset: $7.812 \mathrm{e}-006$

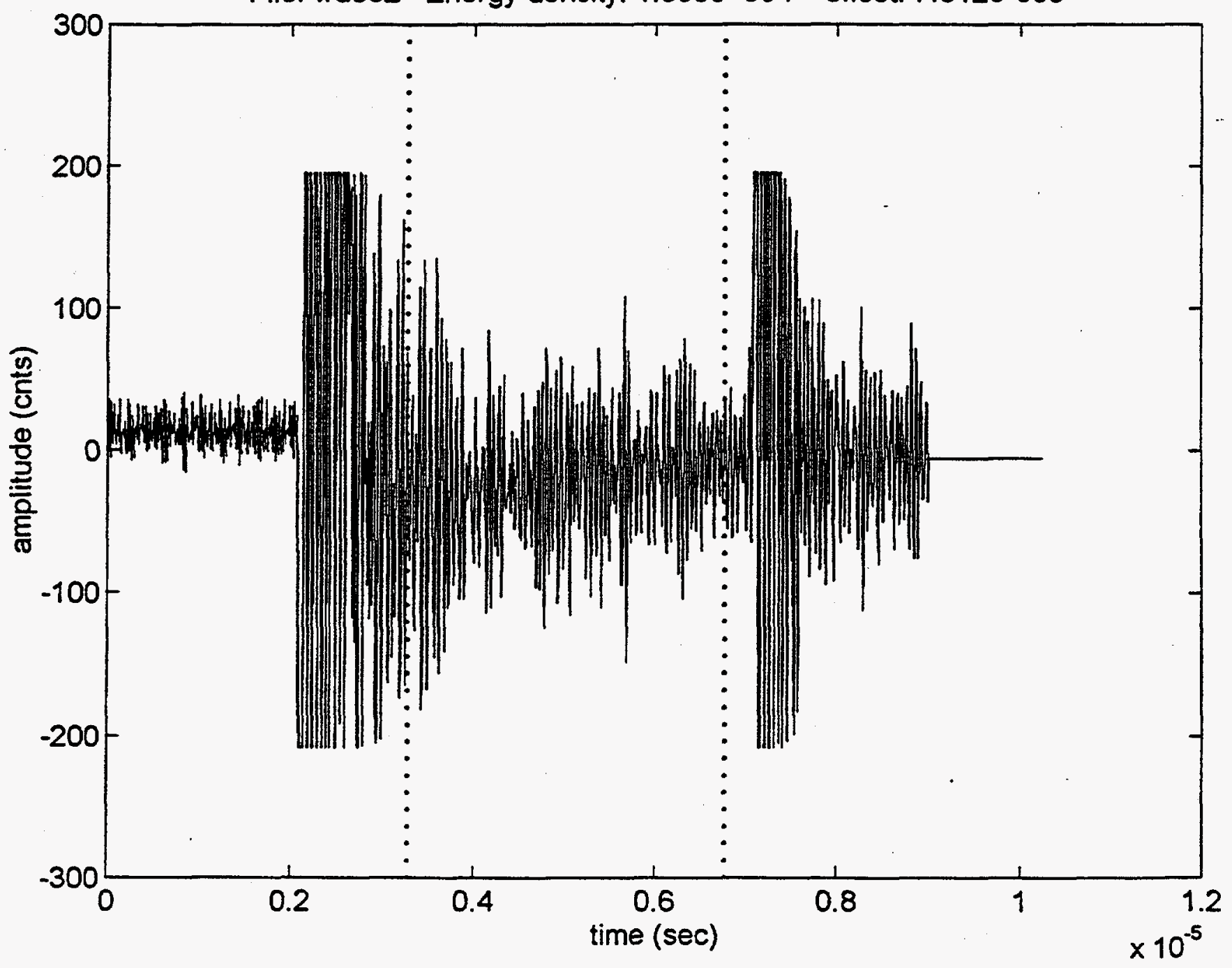


File: wd363 Energy density: $1.493 e+004$ offset: -0.0000459

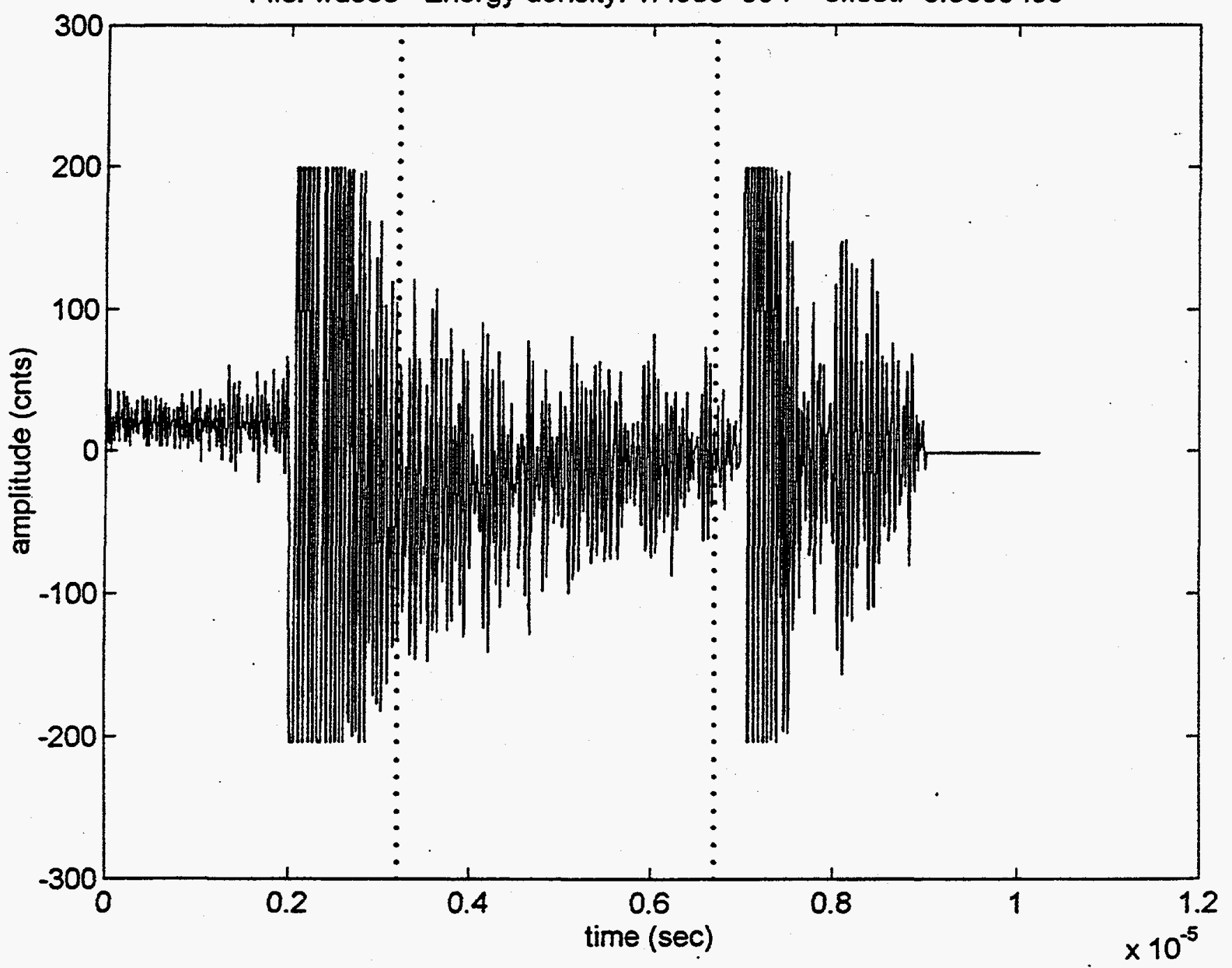


File: wd364 Energy density: $1.662 e+004$ offset: 0.00004395

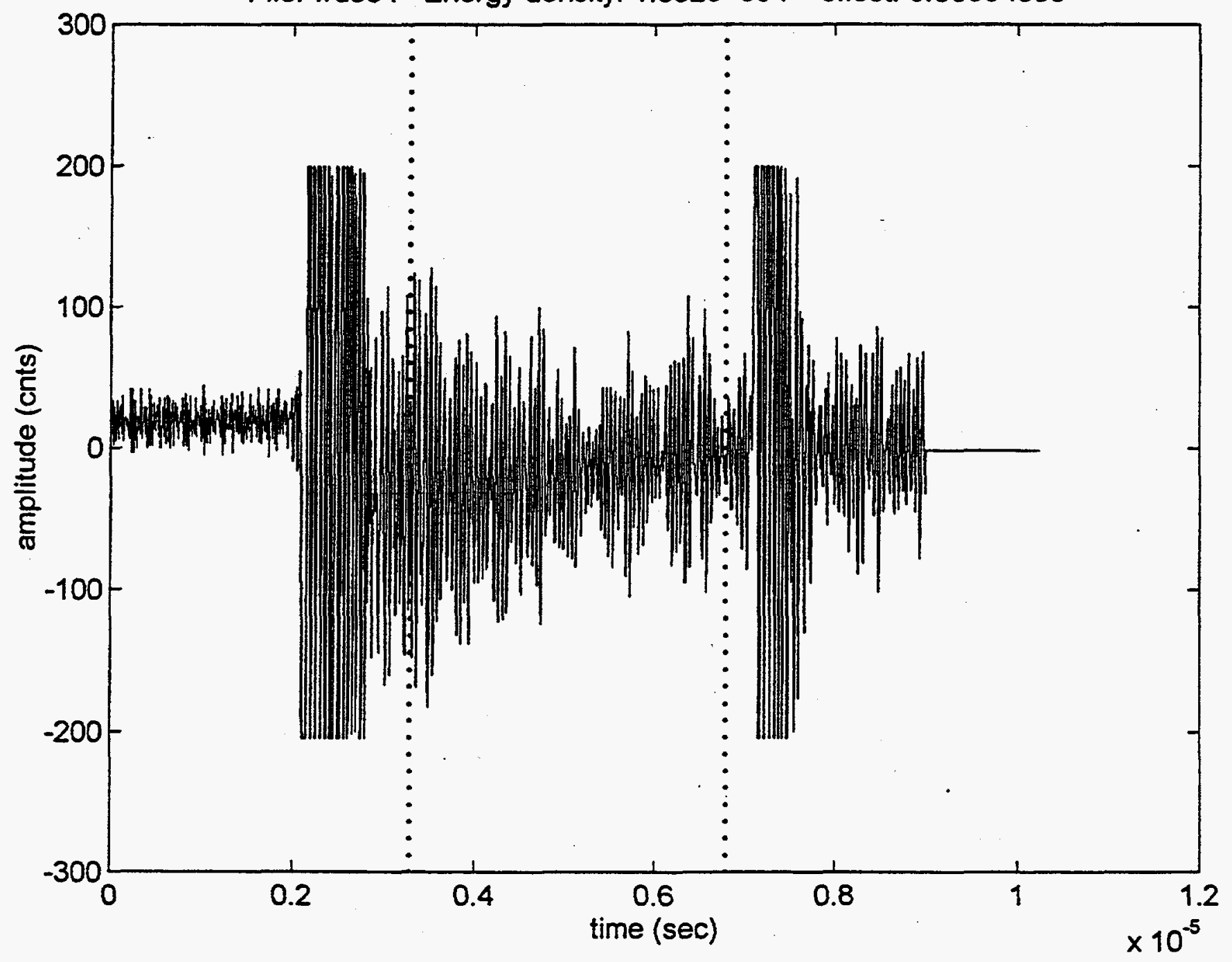




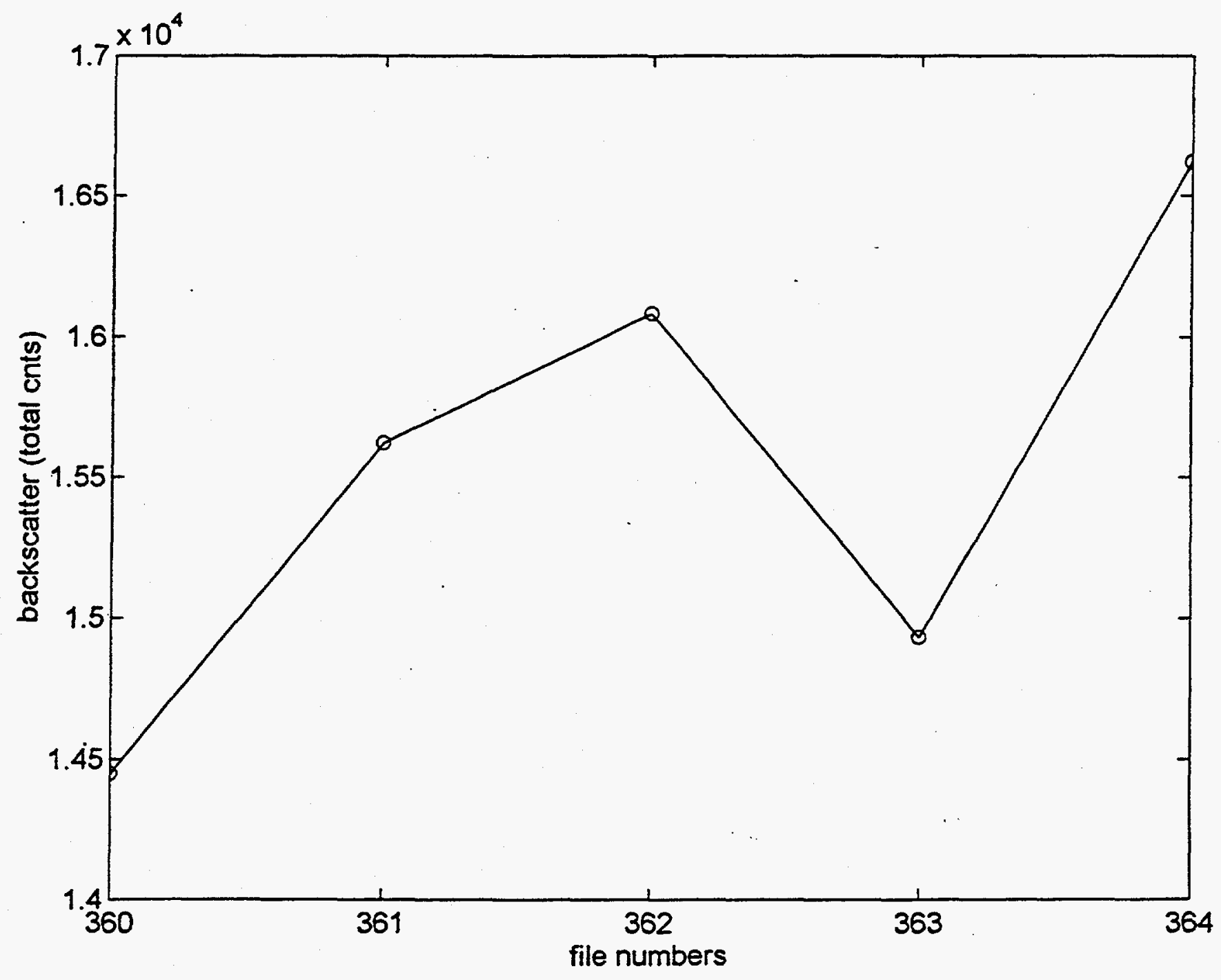




\section{Appendix B Ultrasonic Attenuation Data For Cast Iron Samples}

Appendix B contains a compilation of the ultrasonic attenuation data for the cast steel samples supplied by the American Foundrymen's Society. Provided is a listing of the MATLABTM computer program used to process the data followed sequentially by the raw ultrasonic A-scans and measured attenuation values for each sample. 
\%attnsample.m

$\%$ afunct calculates the attenuation coeff

sand plots the fft and attenuation coeff. for

\%all points of measurement on a given sample (this is a shell)

\%th=input ('thickness $[t 1, t 2] ?$ '):

offset \%subroutine: takes out offset in signals

for $j=1: 2$

if $j==1$, file=sample $(1: 3)$; end

if $j==2$, file=sample $(4: 6)$;end

for $i=1: 3$,

$\%$ freq $=[.1: .1: 1.5] ;$

freg $=[2: .1: 5]$;

$\because$ freq $=\left[\begin{array}{llll}2 & 3 & 4 & 5\end{array}\right]$ :

geval (['load wd', num2str(file(i)),'.txt']):

eval (['ascan=wd', num2str(file (i)), ';']);

ascan=ascan';

ascanall $(:, i)=$ ascan $;$

if $i==1$, attn=file $(i)$; end,

wind

\&subroutine: applies a Hanning window

asfft

\&subroutine: computes fourier transform

eval (['PyY', num2str(i), '=Pyy $(1: 256) ; ']$ );

eval ( [ $B$ ', num2str(i), '=PyY (25.75*freq); ']);

end

clear $i$

time $=1 / 100$ e $*$ [ 1 : length (ascanseg) ] ;

$f f=f(25.75 * f r e q)$;

scale $=\left[\begin{array}{ll}12 & 12\end{array}\right]$;

ascanseq $(1: 256)=\operatorname{ascanal} 1(:, 1) ; \operatorname{ascanseq}(257: 512)=\operatorname{ascanal} 1(:, 2) * \operatorname{sca} 1$

e(1) ; ascanseq $(513: 768)=\operatorname{ascanall}(:, 3) *$ scale $(2)$;

figure(1),

if $j==1$, subplot (211), end

if $j==2$, subplot $(212)$, end

plot (time, ascanseq)

grid,

title(['Ascan of wd', num2str(file(1)), ' wd', num2str(file(2)),' wd' , num2str(file(3)),' (2nd \& 3 rd reflections scaled by ', num2str(sca le (1)),'j'])

xlabel(" scan time (sec)');

ylabel ('Amplitude (arb. units)') 
\&pause

figure (2),

if $j=1$, subplot (211), end

if $j=2$, subplot $(212)$, end

plot (f, [PYY1 PYY2*200 PyY3*200], ff, [B1 B2*200 B3*200], 'O')

grid,

title(['Fourier transform of wd',num2str(file(1)),' wd', num2str(fi le(2)),' wd', numzstr(file(3)),' (2nd \& 3rd transformations scaled by 200)'])

xlabel ('Frequency $(\mathrm{Hz})$ '):

ylabel ('Amplitude (arb. units)')

ะpause

$\operatorname{disp}(a t t n)$

$d=\operatorname{th}(j)$;

attncoef

\%subroutine: calculates attenuation coeffi

cient

eval (['attn', num2str (attn), '=a; ']) ;

\%eval (['save temp attn', num2str(attn), '; ] );

\%clear

\%load temp

\%load atten

\%save atten

end

pause

eval (['al=attn', num2str (sample (1)), '; ] ) ;

eval ('['az=attn', num2str(sample (4)), '; ']) ;

figure (3),

plot (ff,al, ff,al, 'o', ff,a2,ff,a2, '*'), grid,

title(['Attenuation Coefficients for sample ', sampnm,', position $A$ \& $\left.\left.B^{\prime}\right]\right)$;

xlabel ('o-position A, *-position B,

Frequency $\left.(\mathrm{Hz})^{\prime}\right)$;

ylabel ('Atten. Coef. (nepers/mm)');

\%text $\left(6 e 6, .15\right.$, 'o-position $\left.A^{\prime}\right)$;

\%text $\left(6 e 6, .14,{ }^{\prime} *\right.$-position $\left.B^{\prime}\right)$;

\%pause

figure (1), print

figure (2), print

figure (3),print 
OFFSET.M

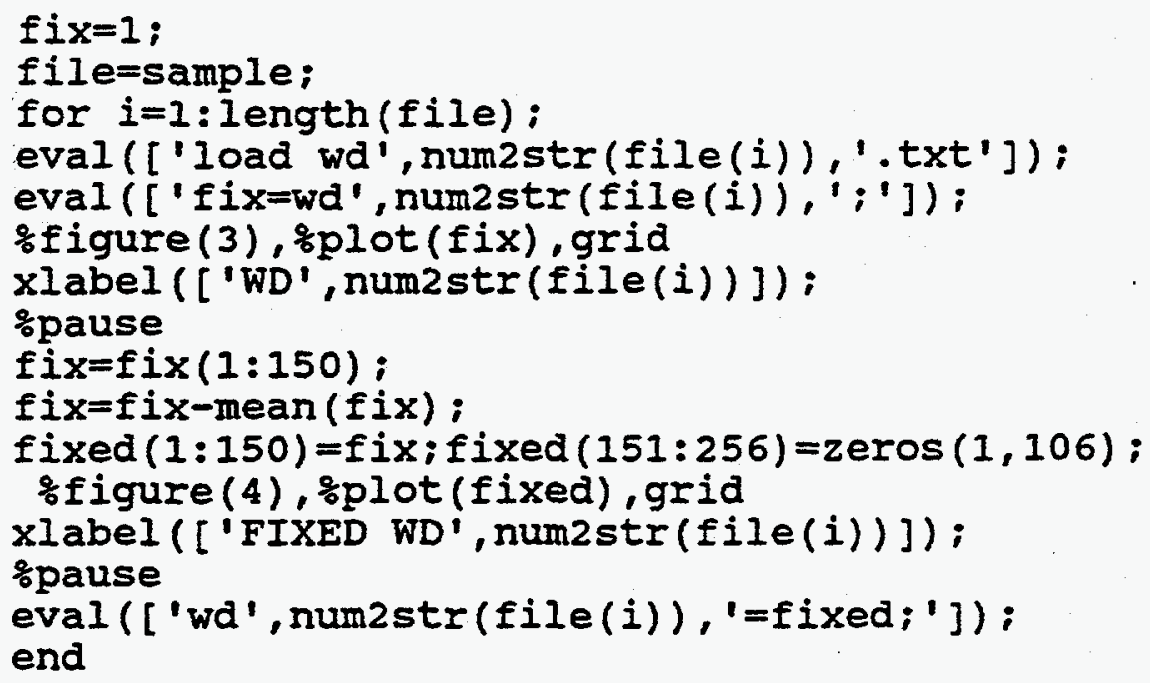


WIND.M

\%set up window for ascan data

figure(3),plot(ascan), grid

$[a, b]=\operatorname{ginput}(1)$;

pt=ceil (a):

w=hanning (128);

ascan $=\operatorname{ascan}(p t-63: p t+64) . * w$;

figure (3), plot(ascan), grid 
sascan fourier transform

$Y=f f t(\operatorname{ascan}, 512) ;$

PYY $=Y . *$ conj $(Y) / 512$;

$f=100 e 6 *(0: 255) / 512$;

\%plot (f, PyY (1:256)) 

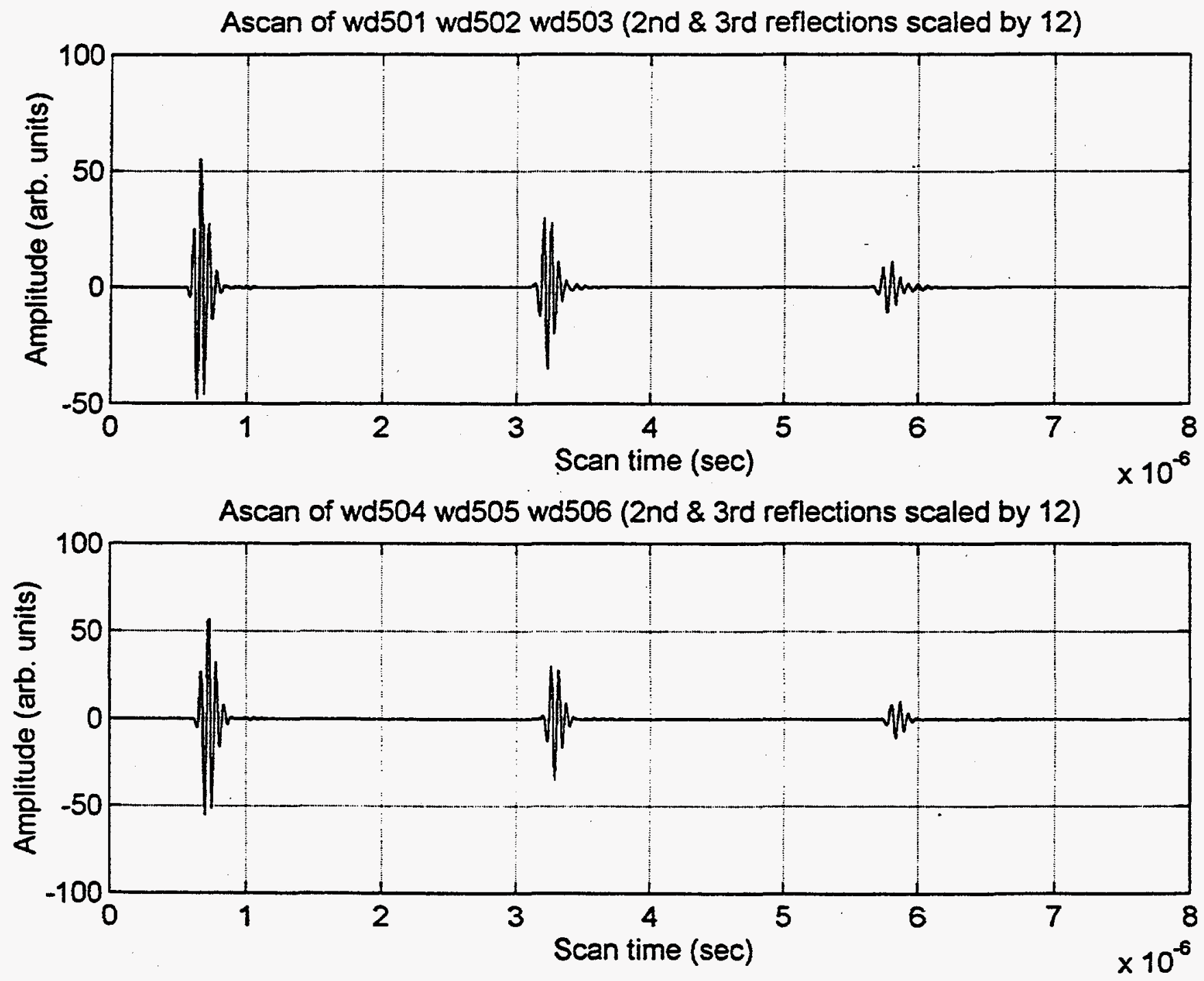
Fourier transform of wd501 wd502 wd503 (2nd \& 3rd transformations scaled by 200)

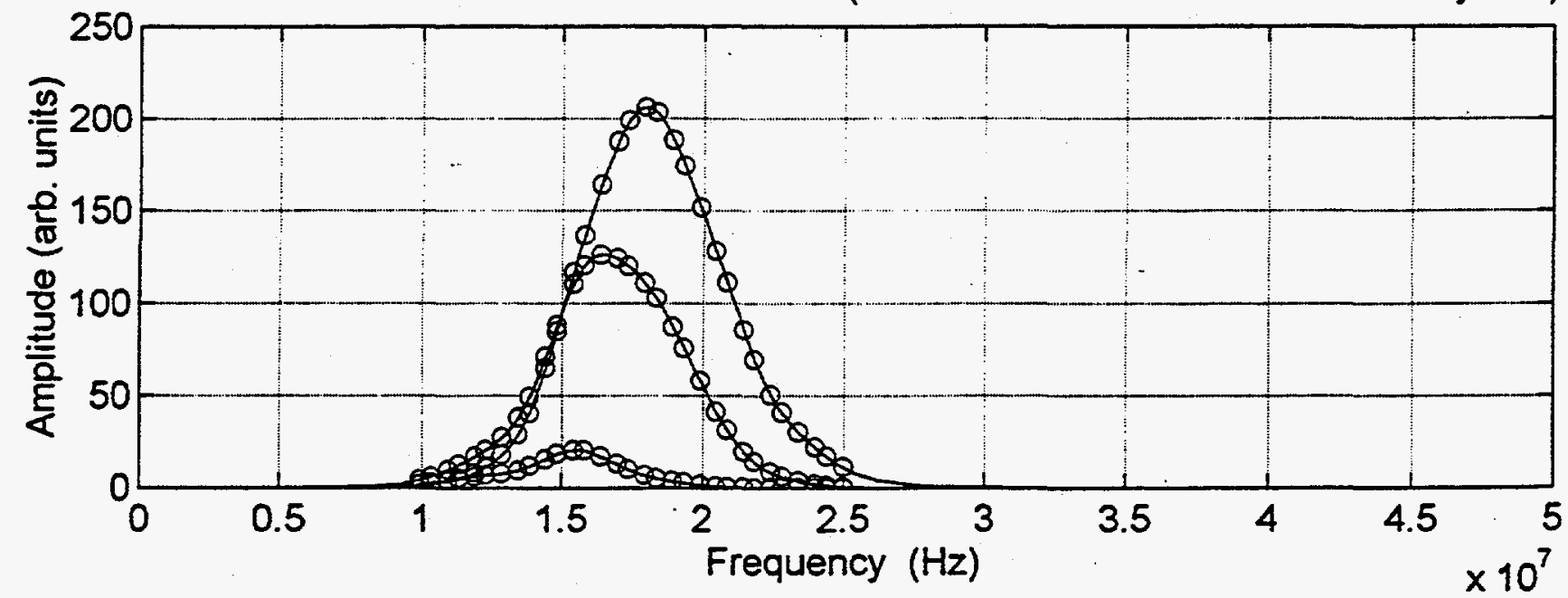

Fourier transform of wd504 wd505 wd506 ( 2 nd \& 3rd transformations scaled by 200 )

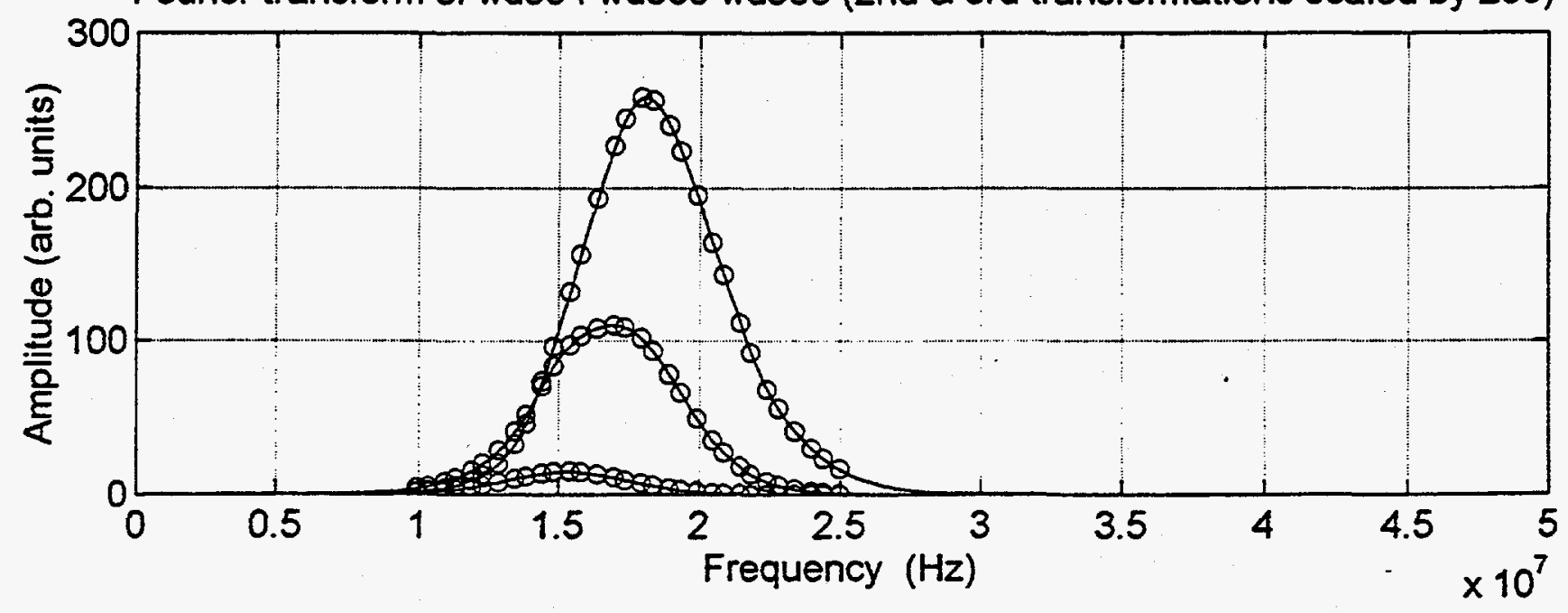




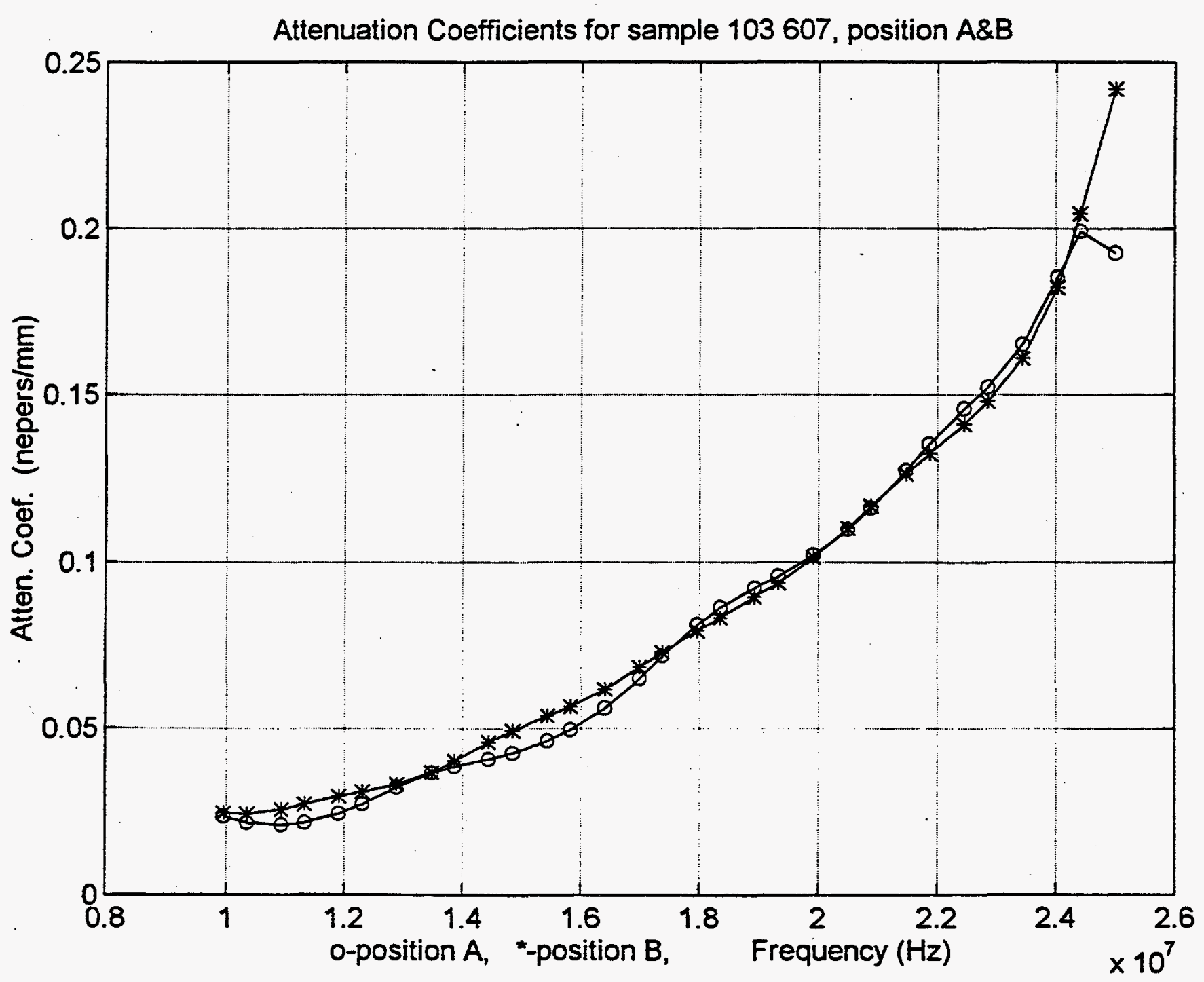



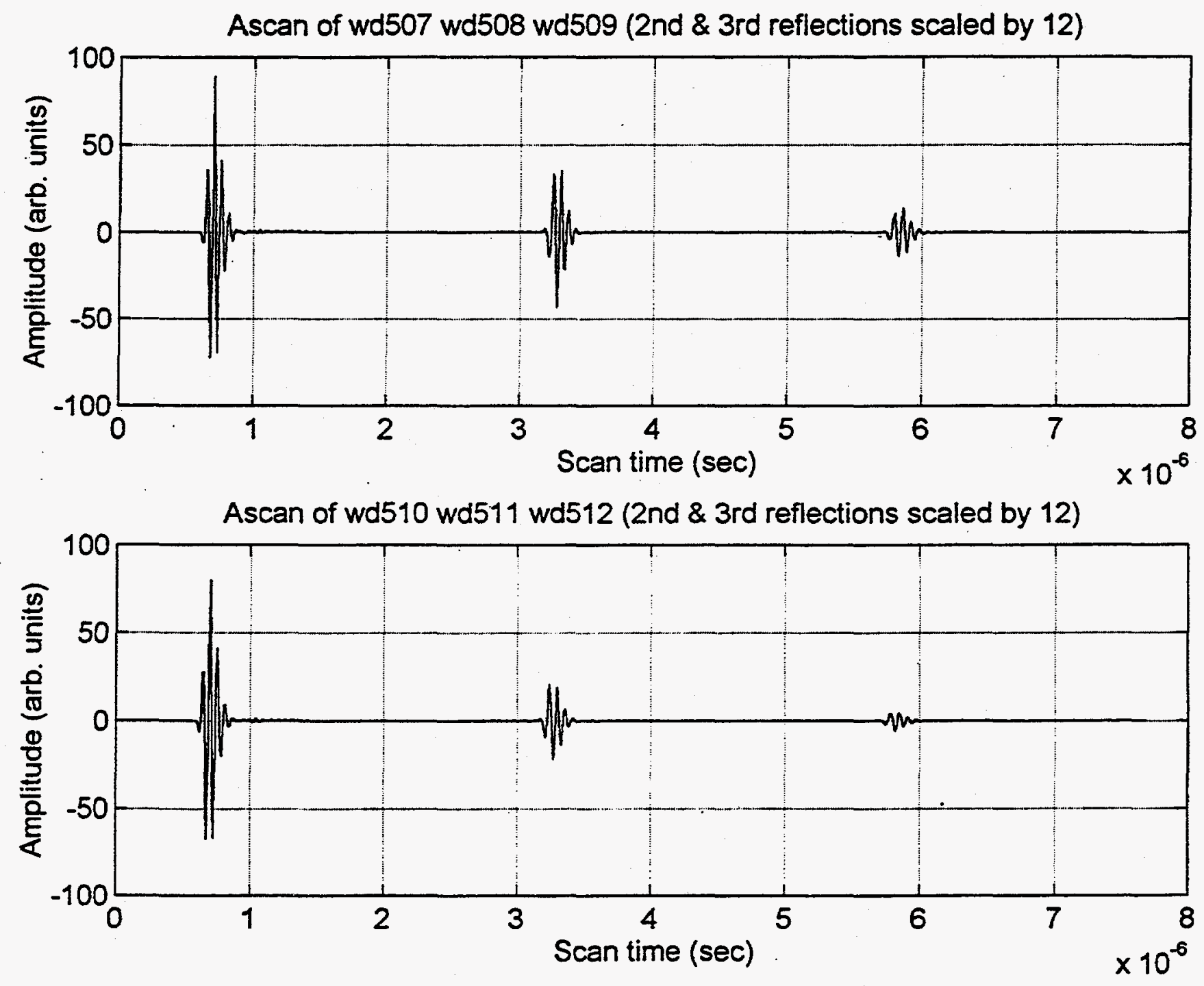

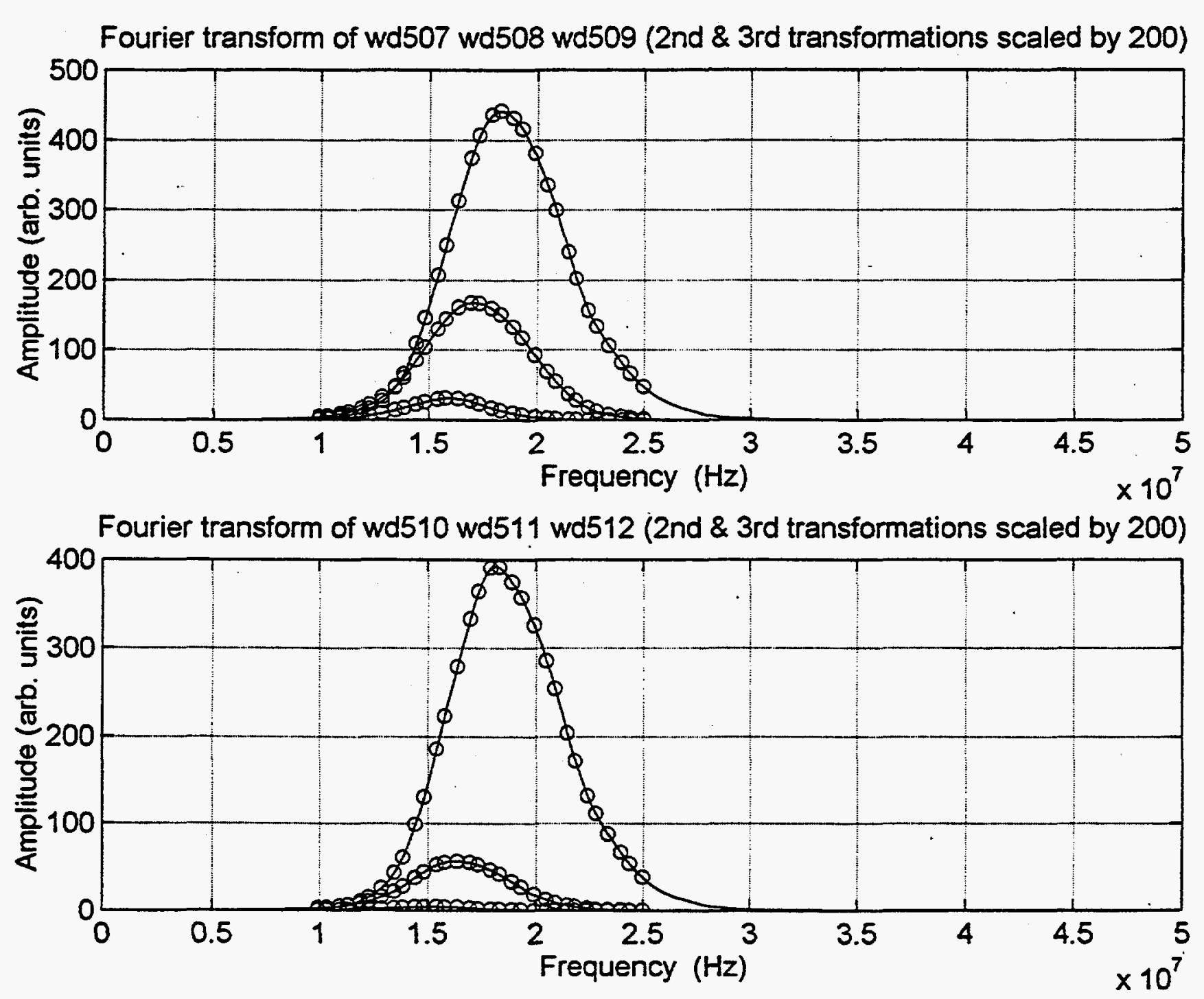


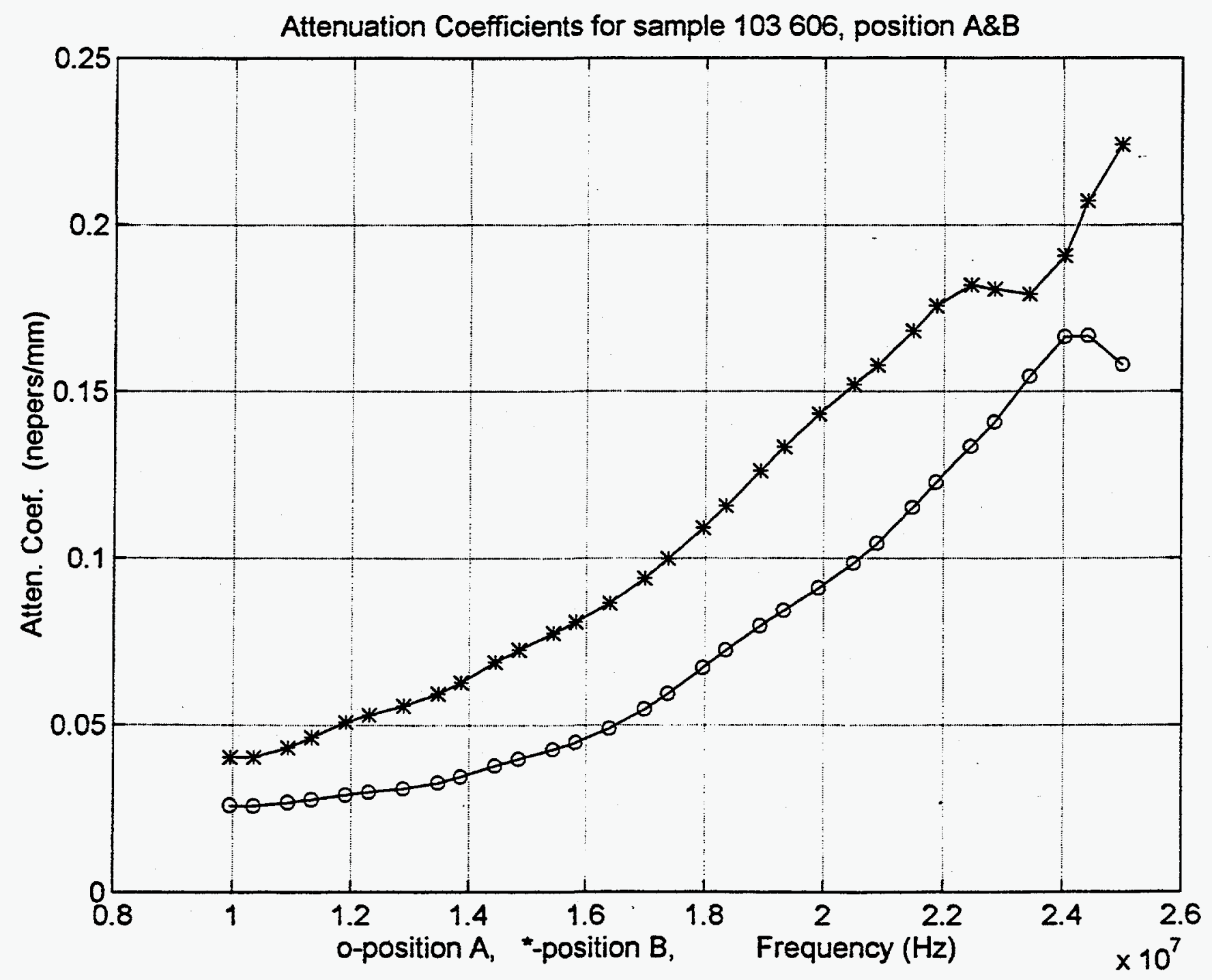



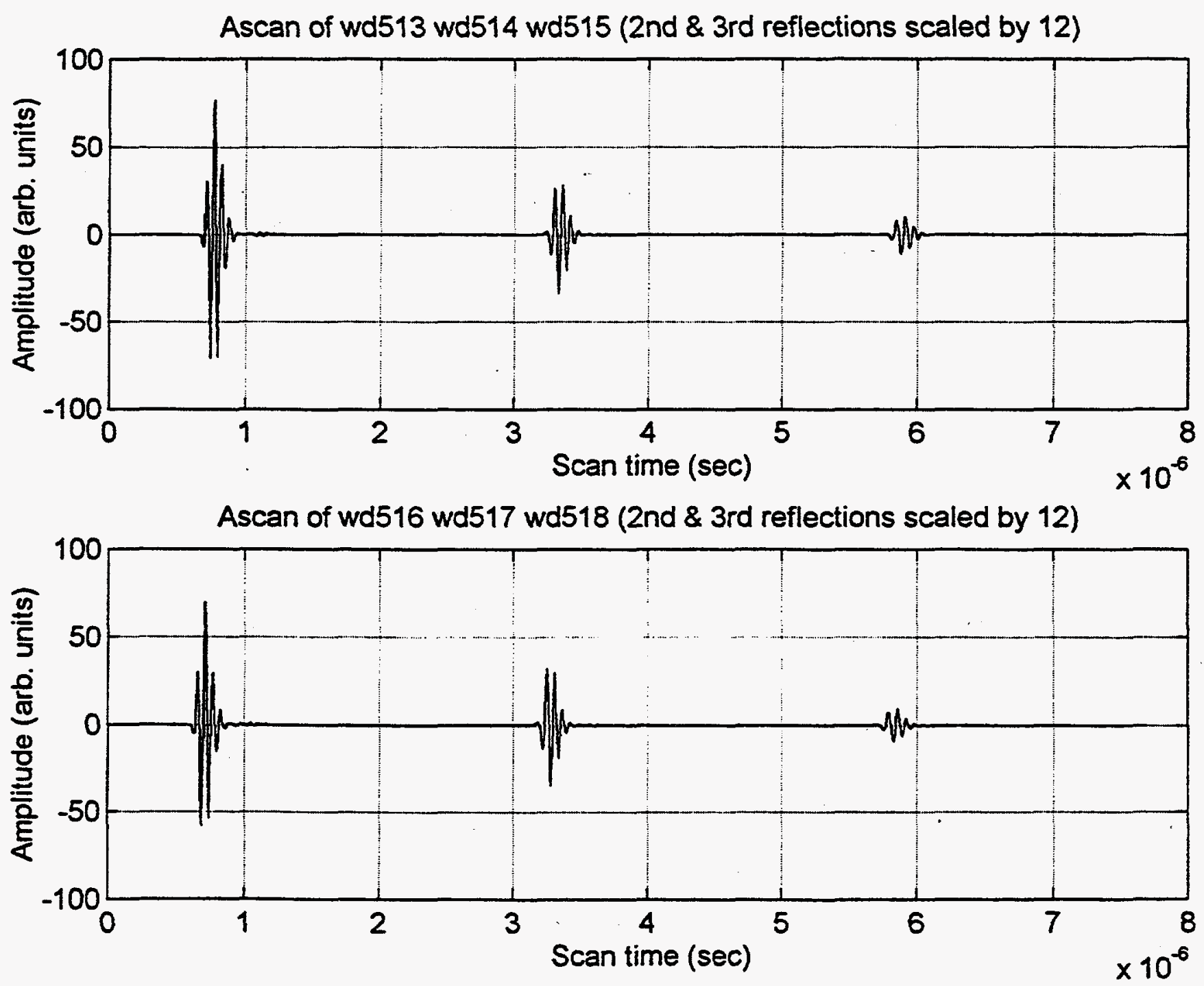


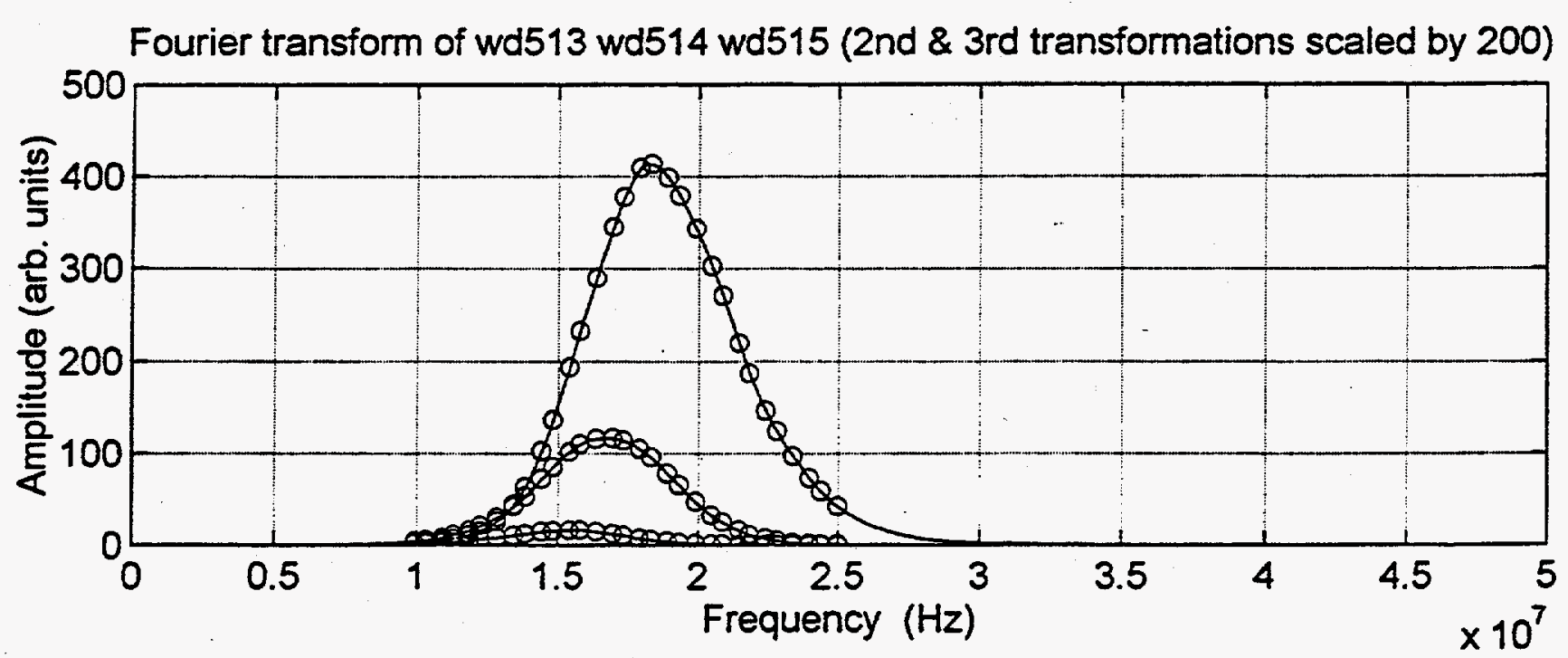

Fourier transform of wd516 wd517 wd518 ( 2 nd \& 3rd transformations scaled by 200 )

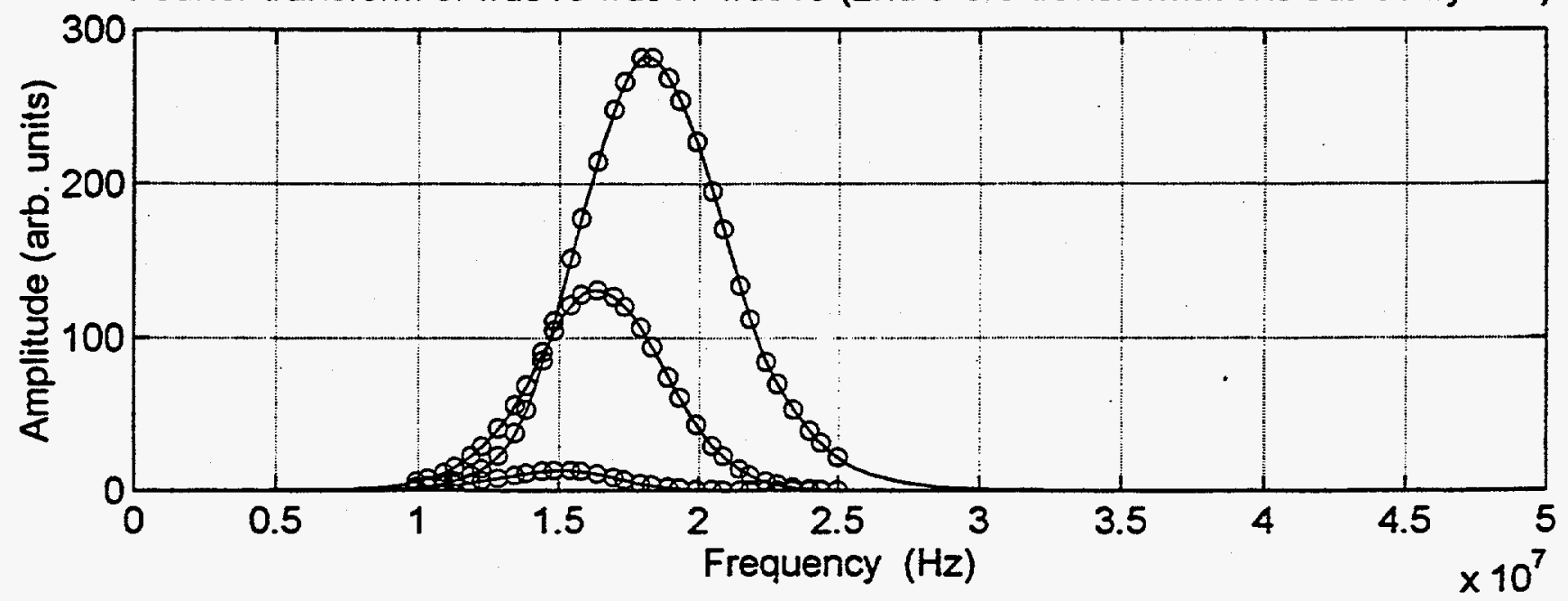




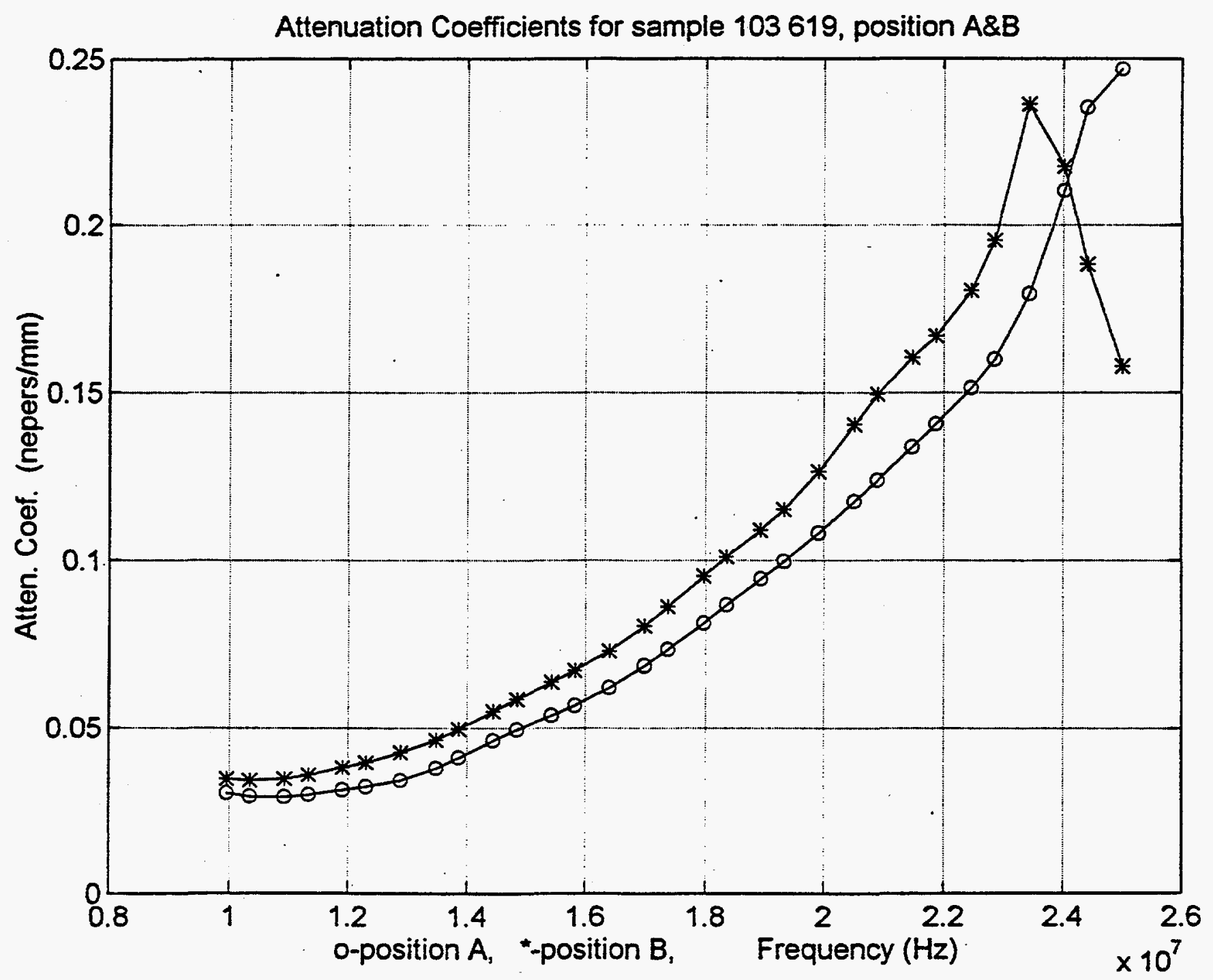



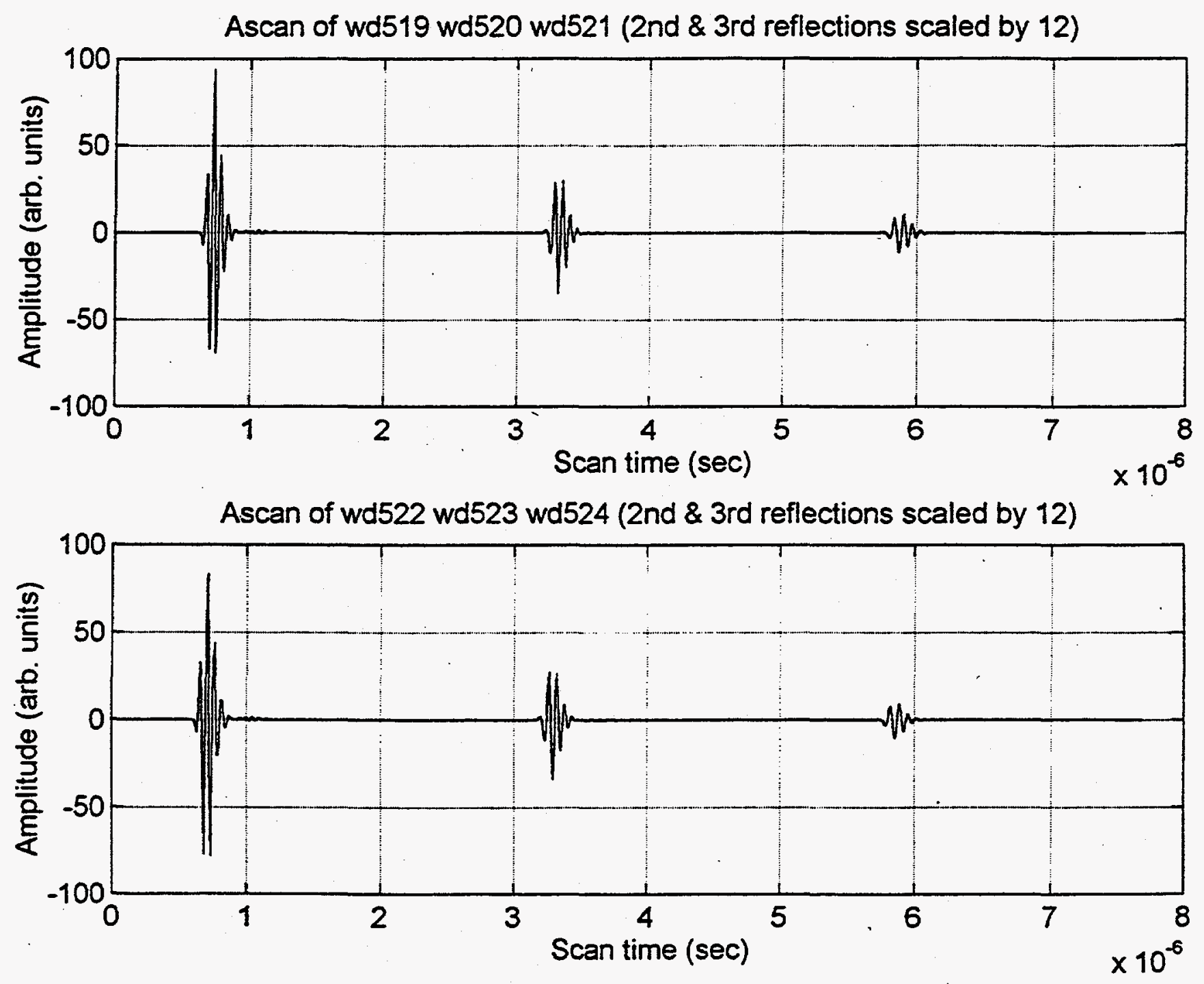
Fourier transform of wd519 wd520 wd521 (2nd \& 3rd transformations scaled by 200)
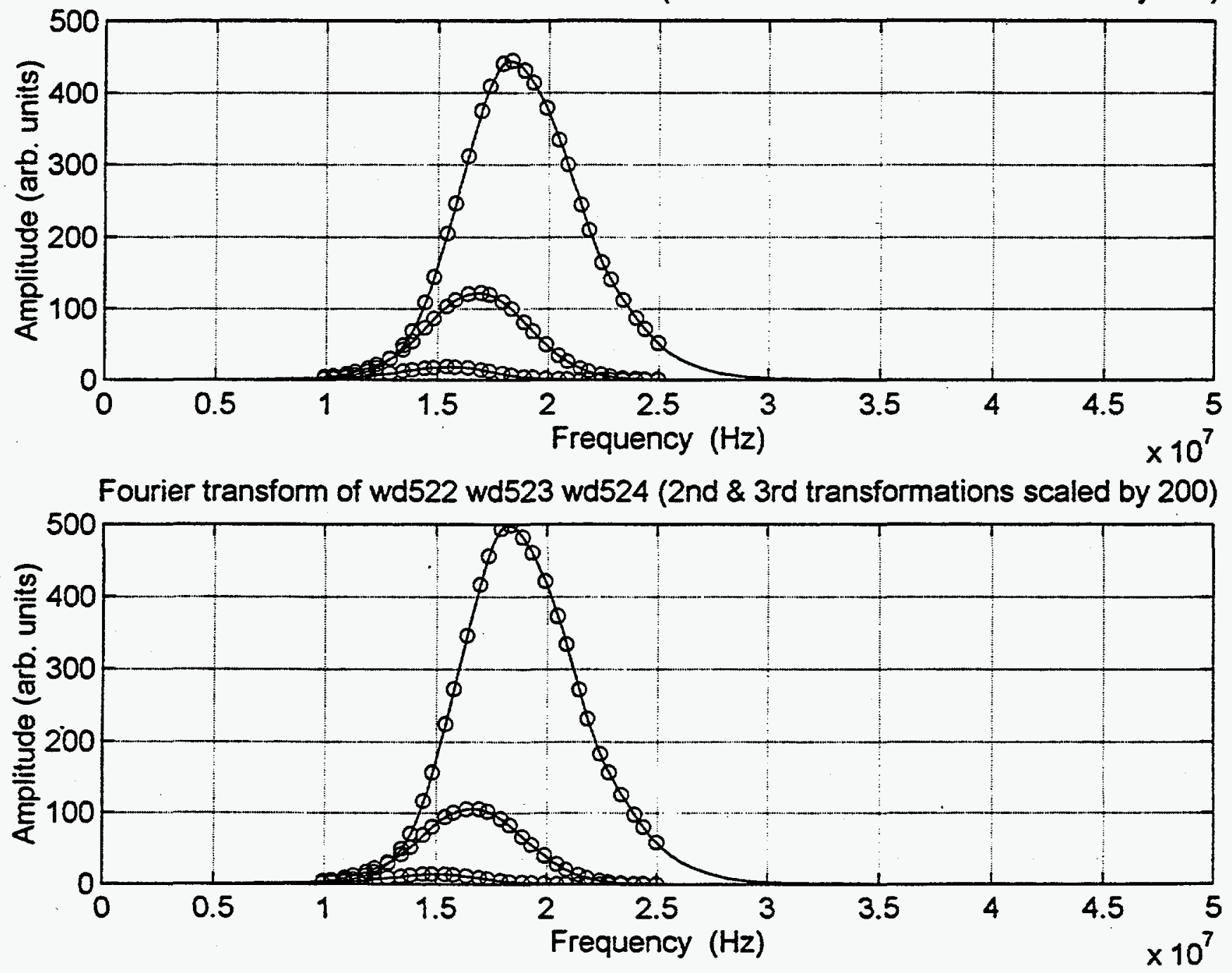


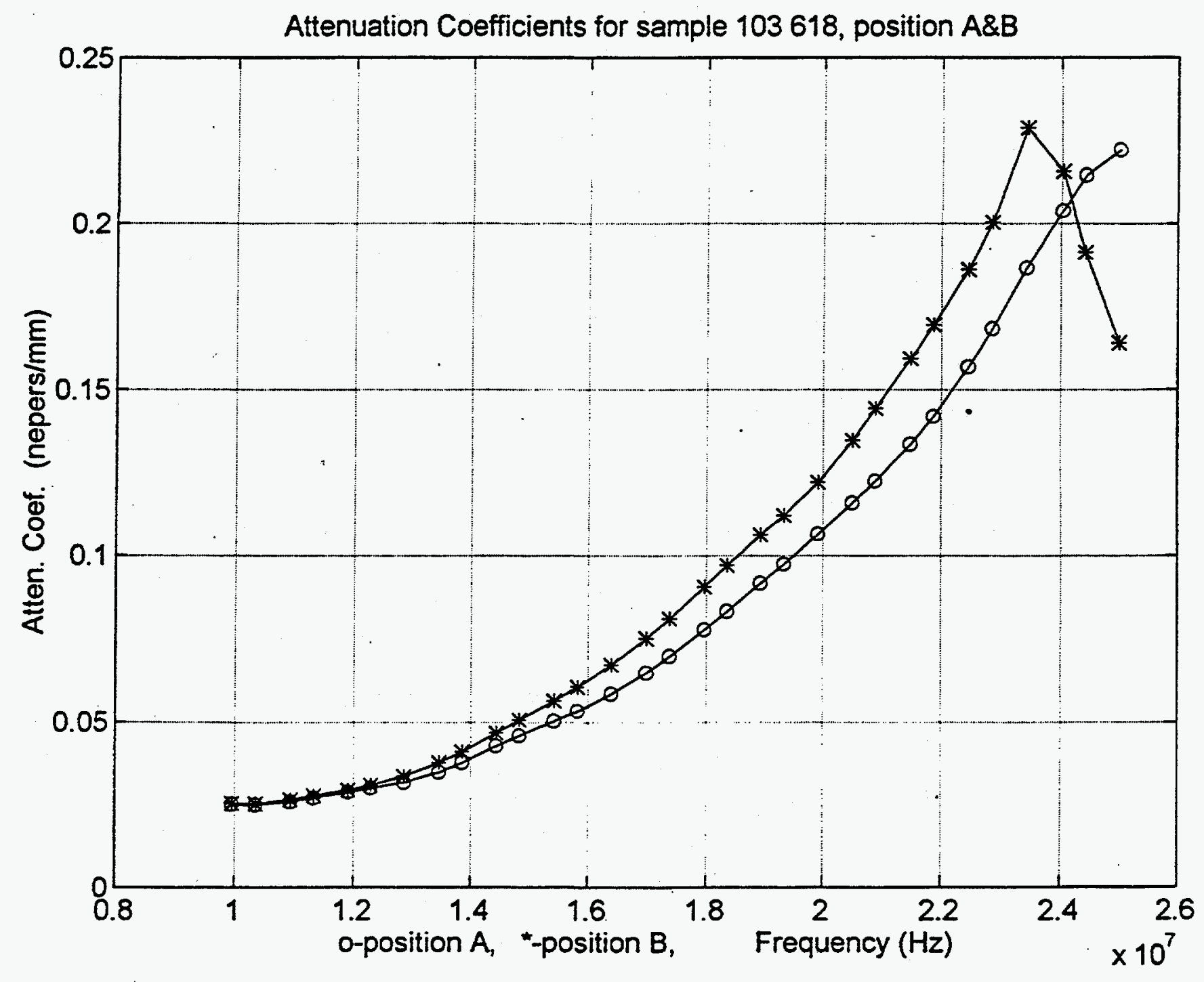


Ascan of wd531 wd532 wd533 ( 2 nd \& 3rd reflections scaled by 12)

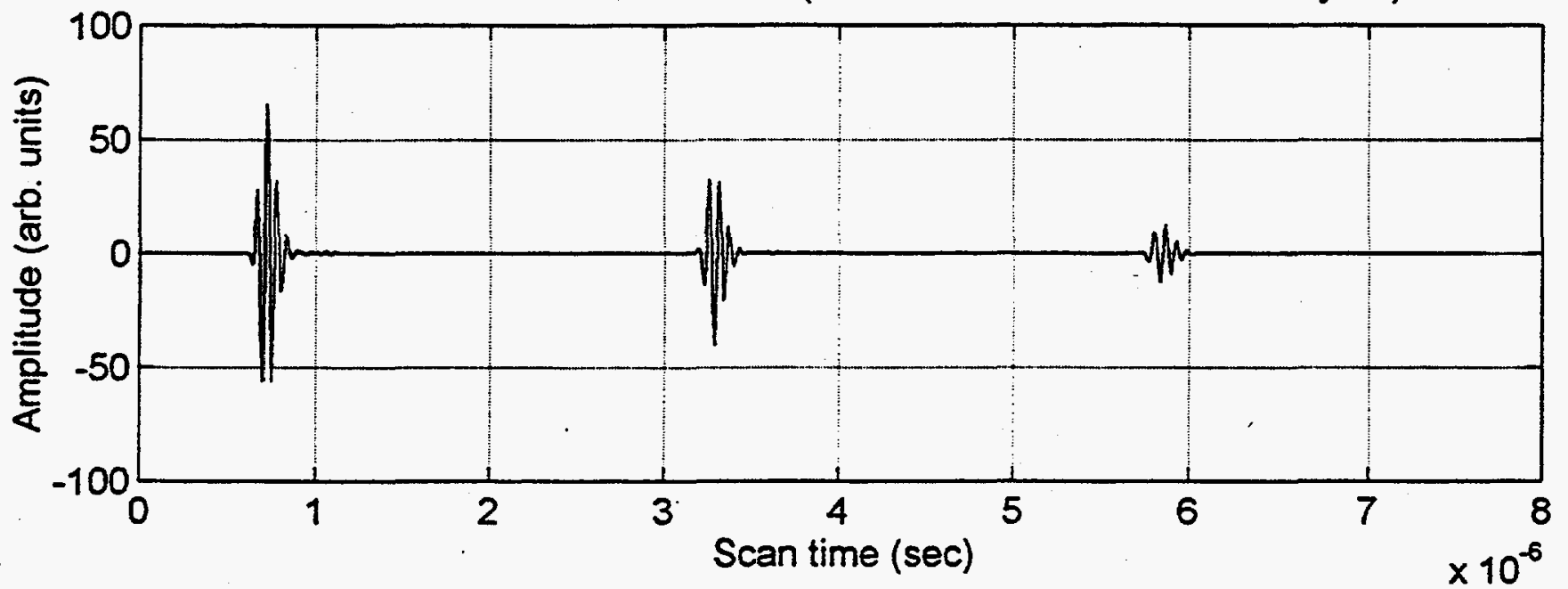

Ascan of wd534 wd535 wd536 (2nd \& 3rd reflections scaled by 12)

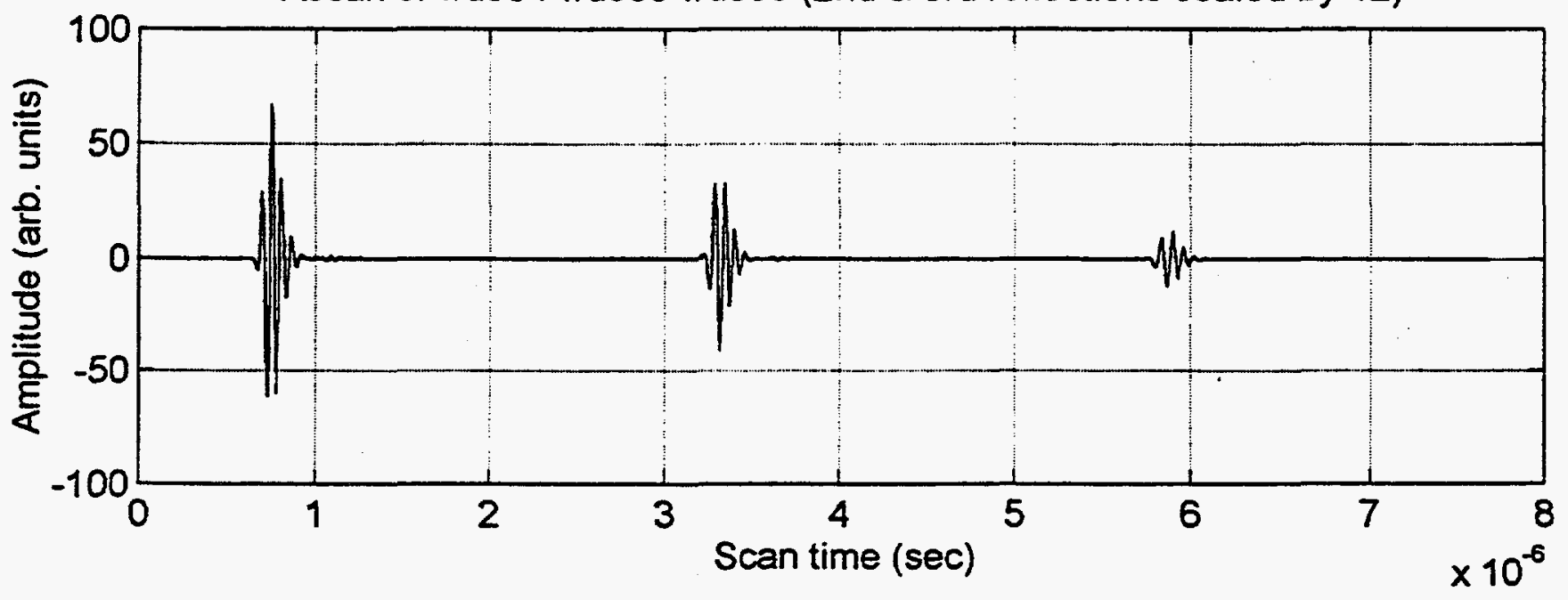


Fourier transform of wd531 wd532 wd533 (2nd \& 3rd transformations scaled by 200 )

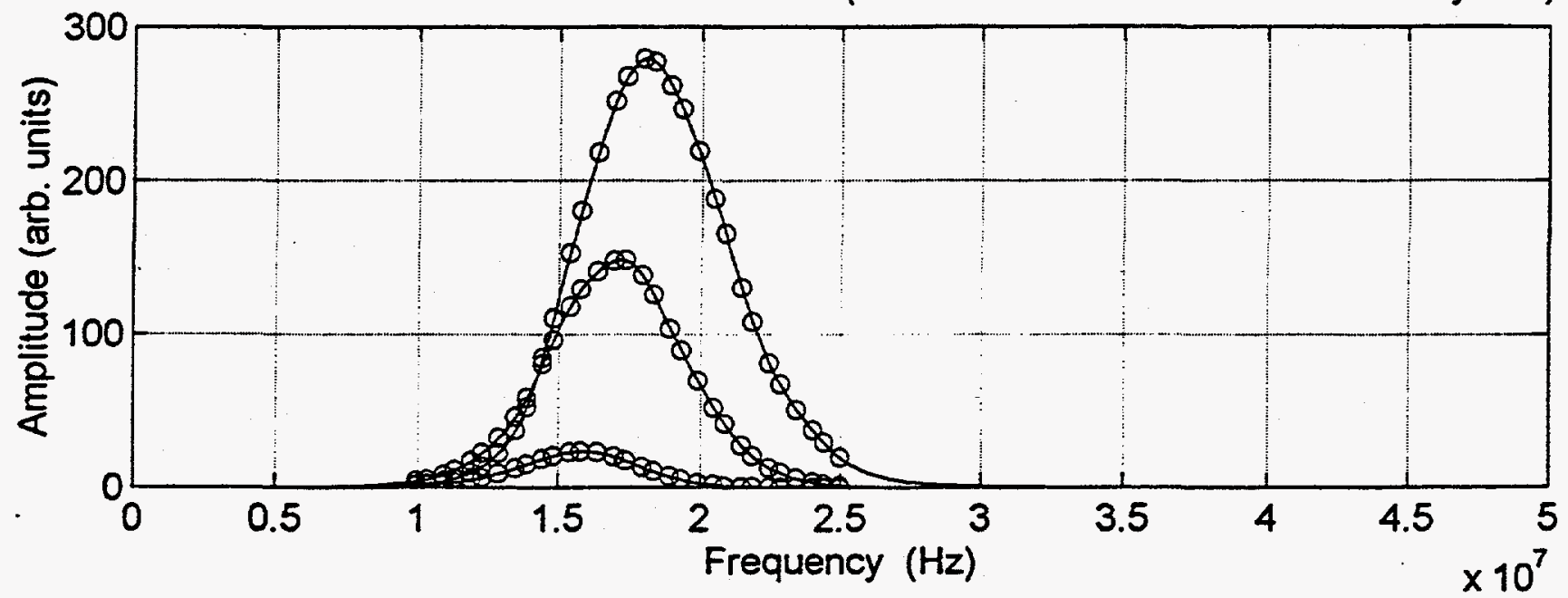

Fourier transform of wd534 wd535 wd536 ( 2 nd \& 3rd transformations scaled by 200 )

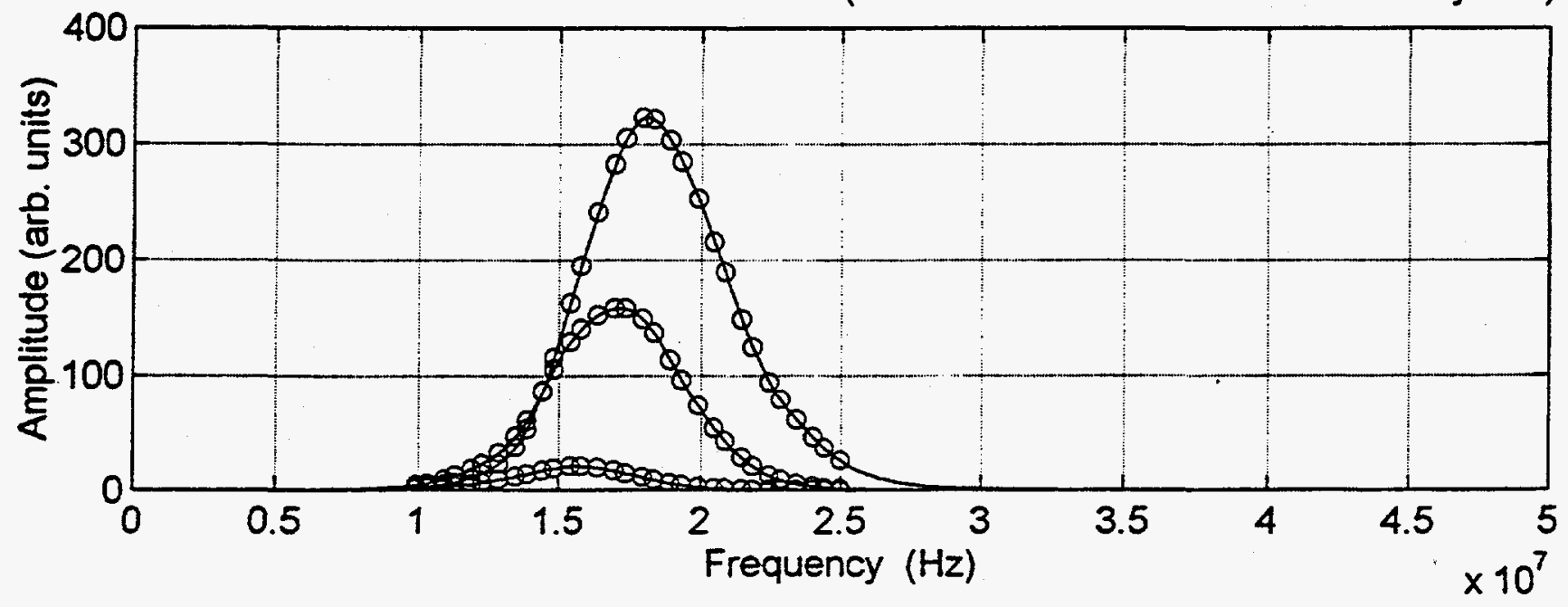




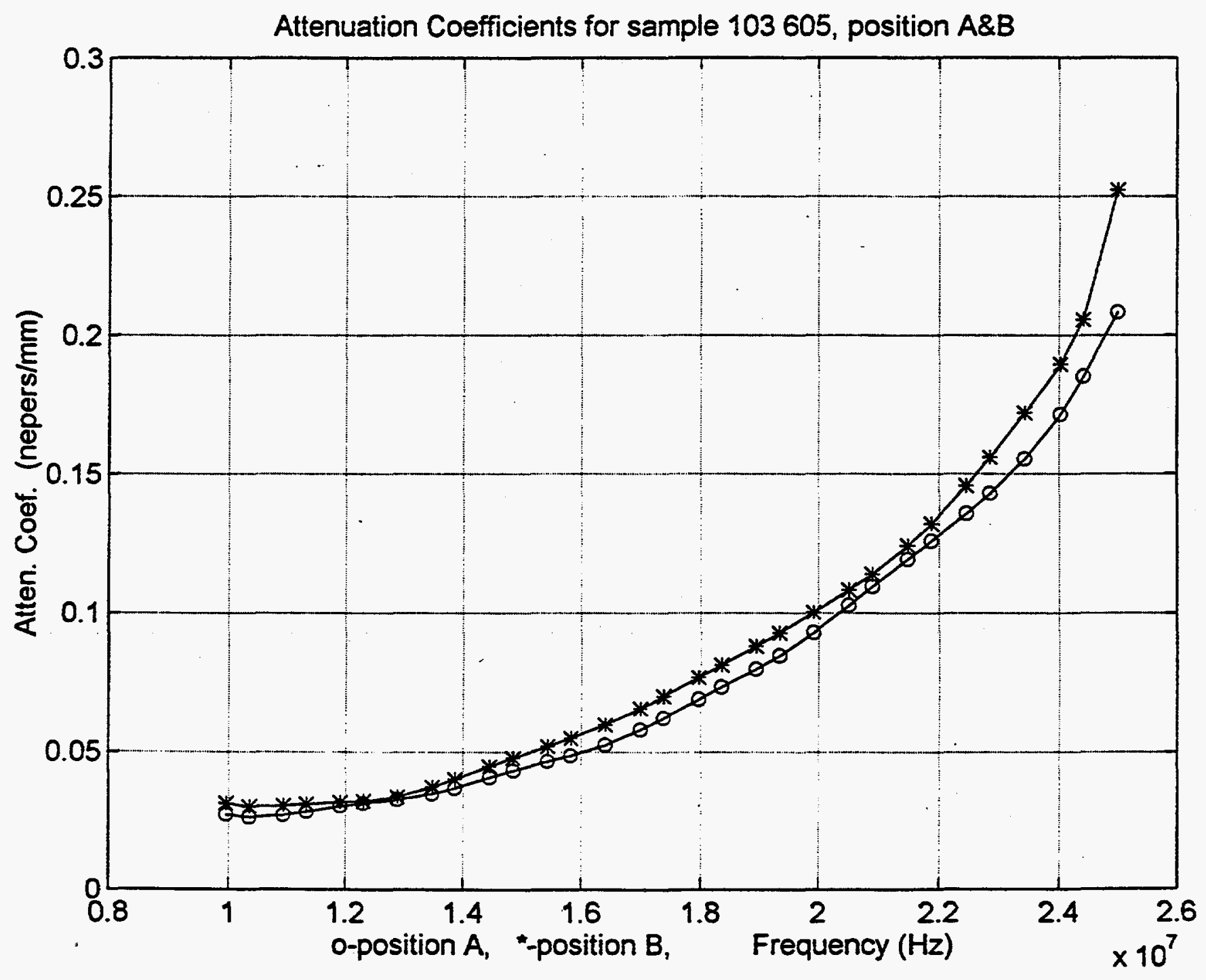



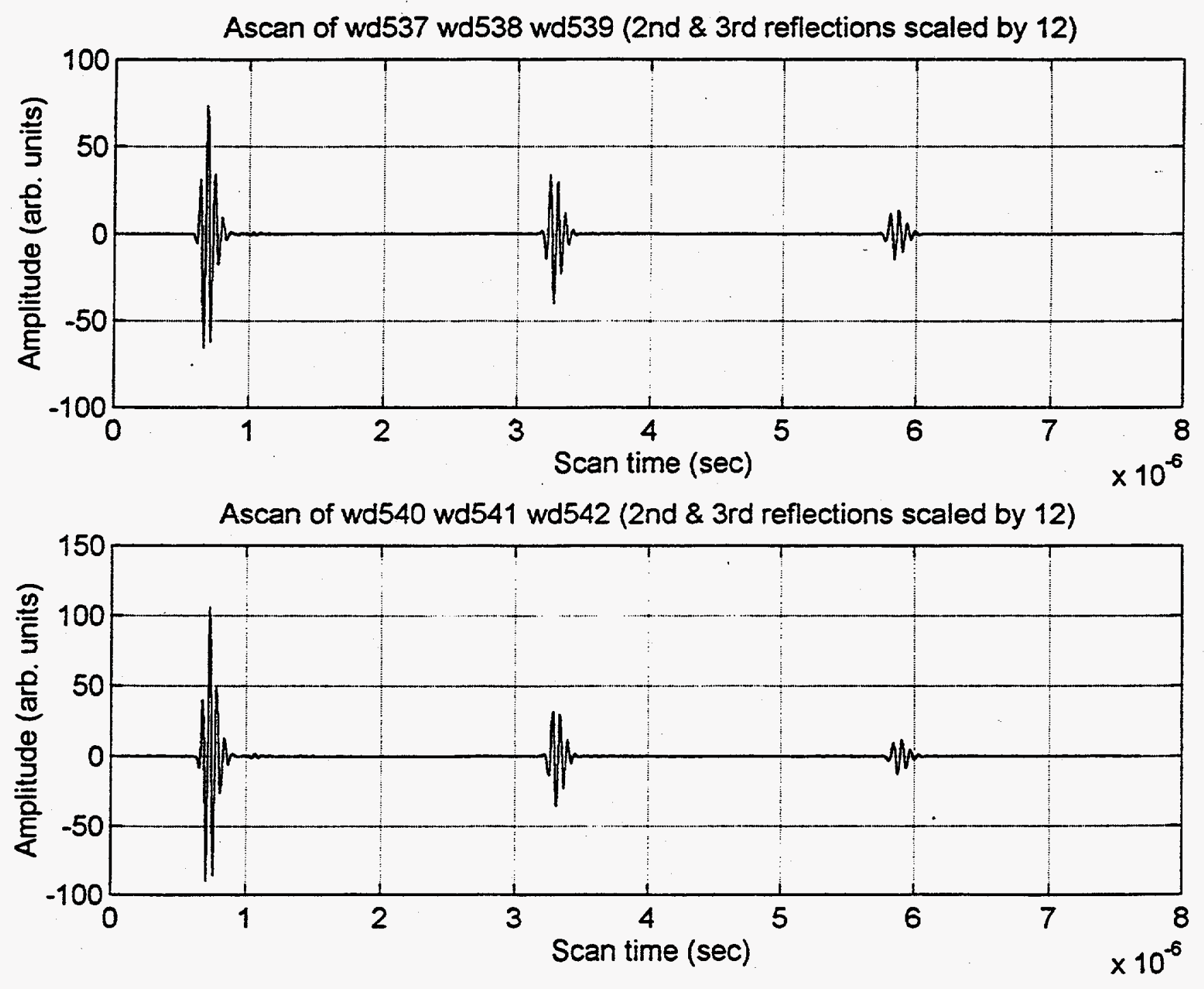


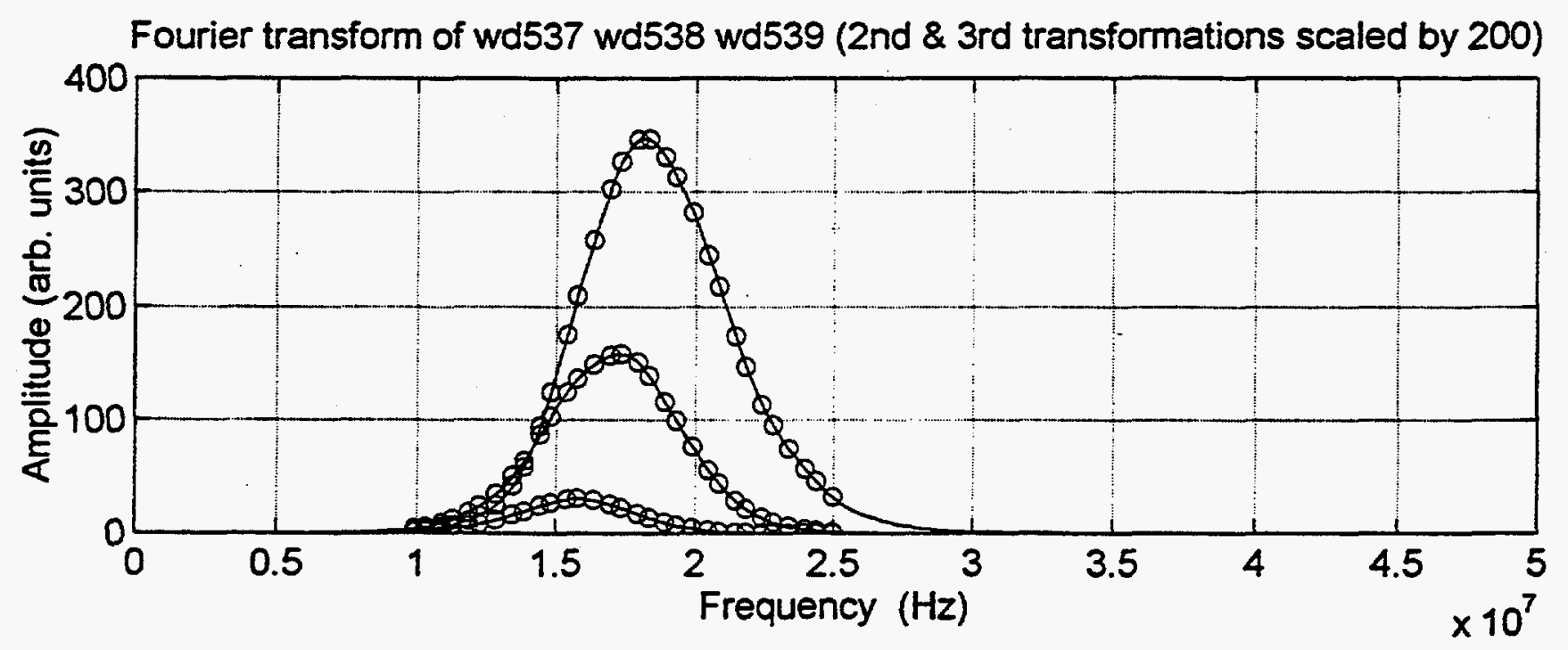

Fourier transform of wd540 wd541 wd542 ( $2 \mathrm{nd} \&$ 3rd transformations scaled by 200 )

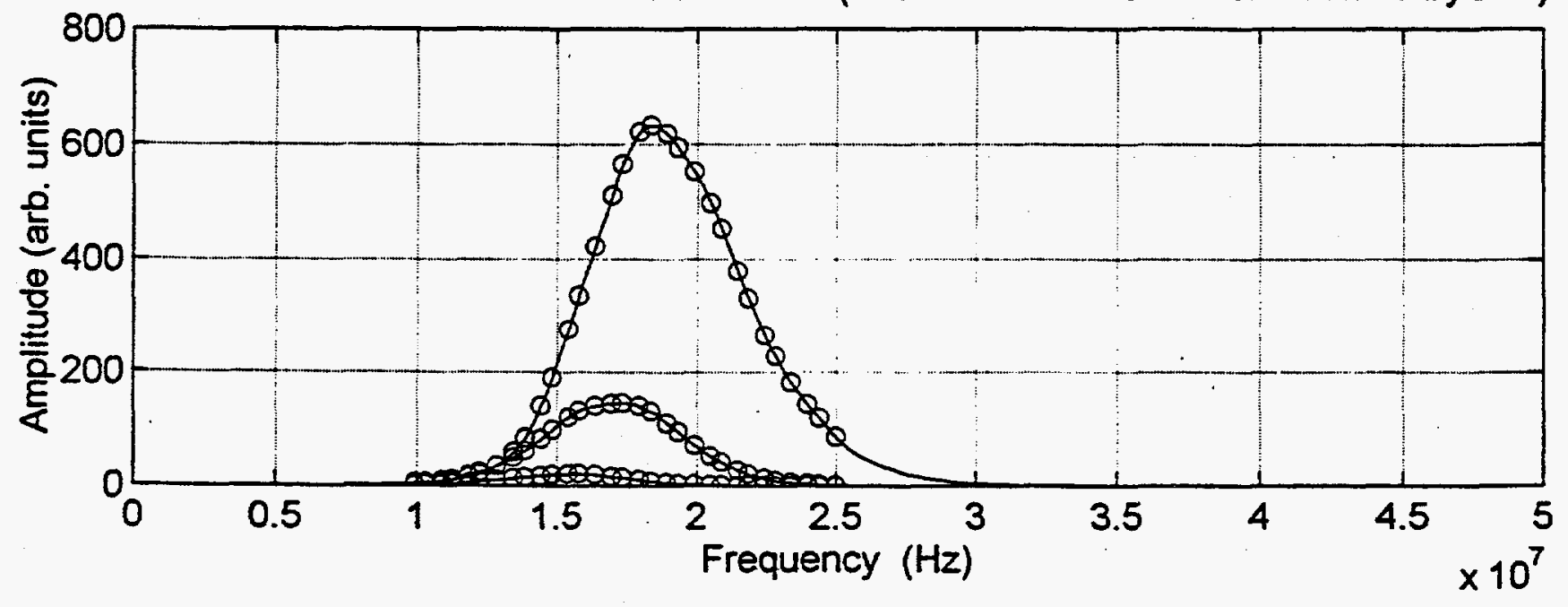




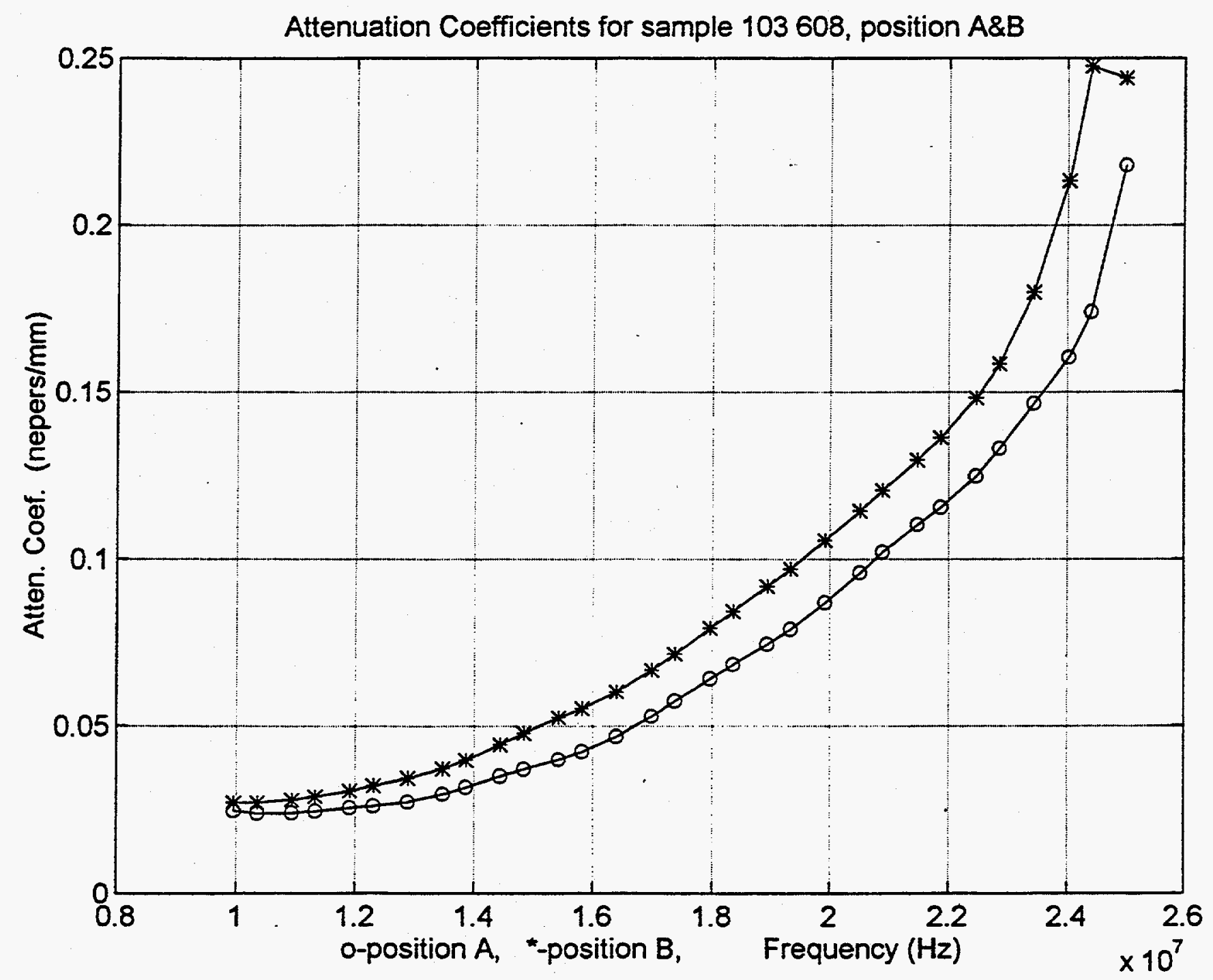



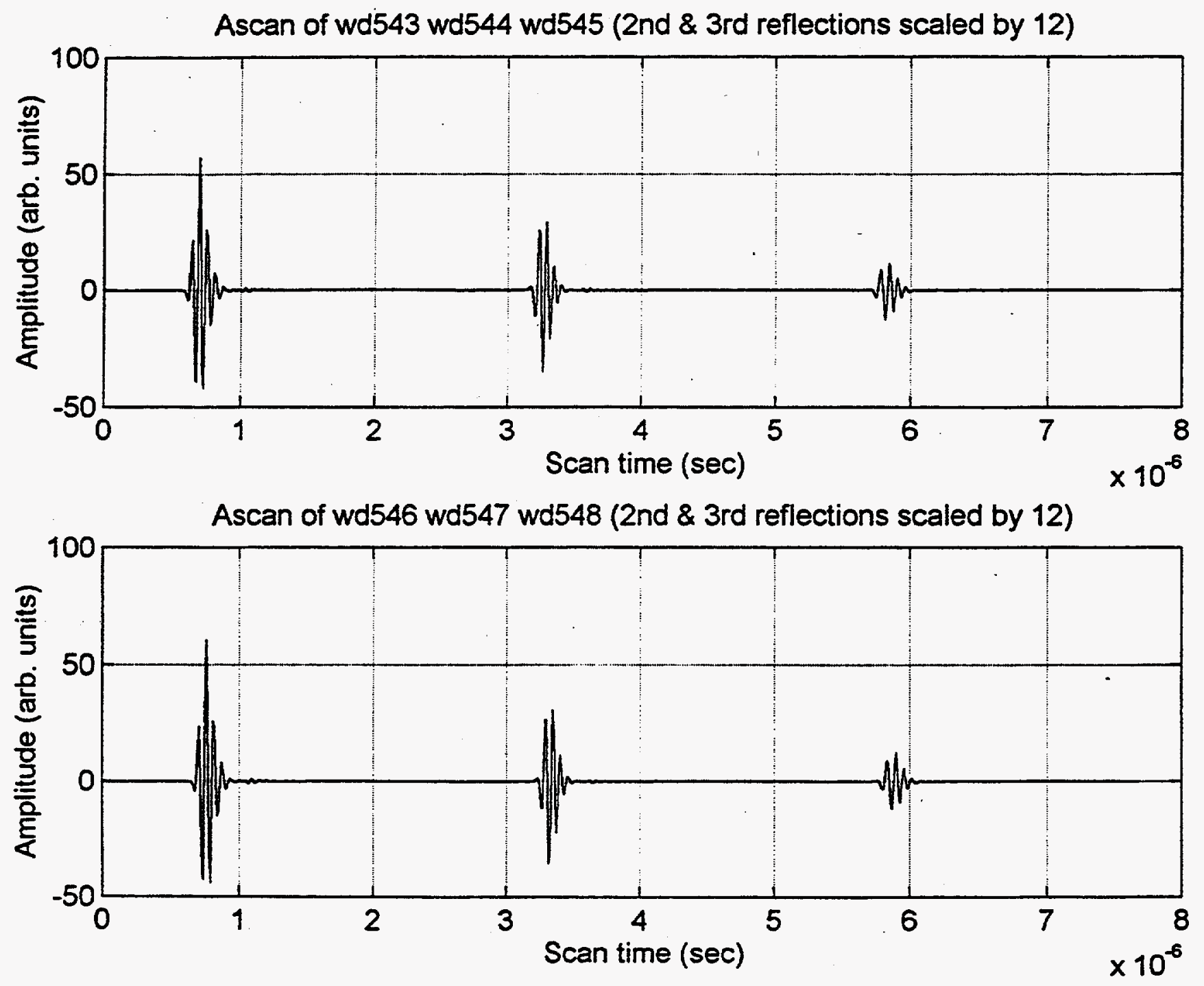
Fourier transform of wd543 wd544 wd545 ( 2 nd \& 3rd transformations scaled by 200)

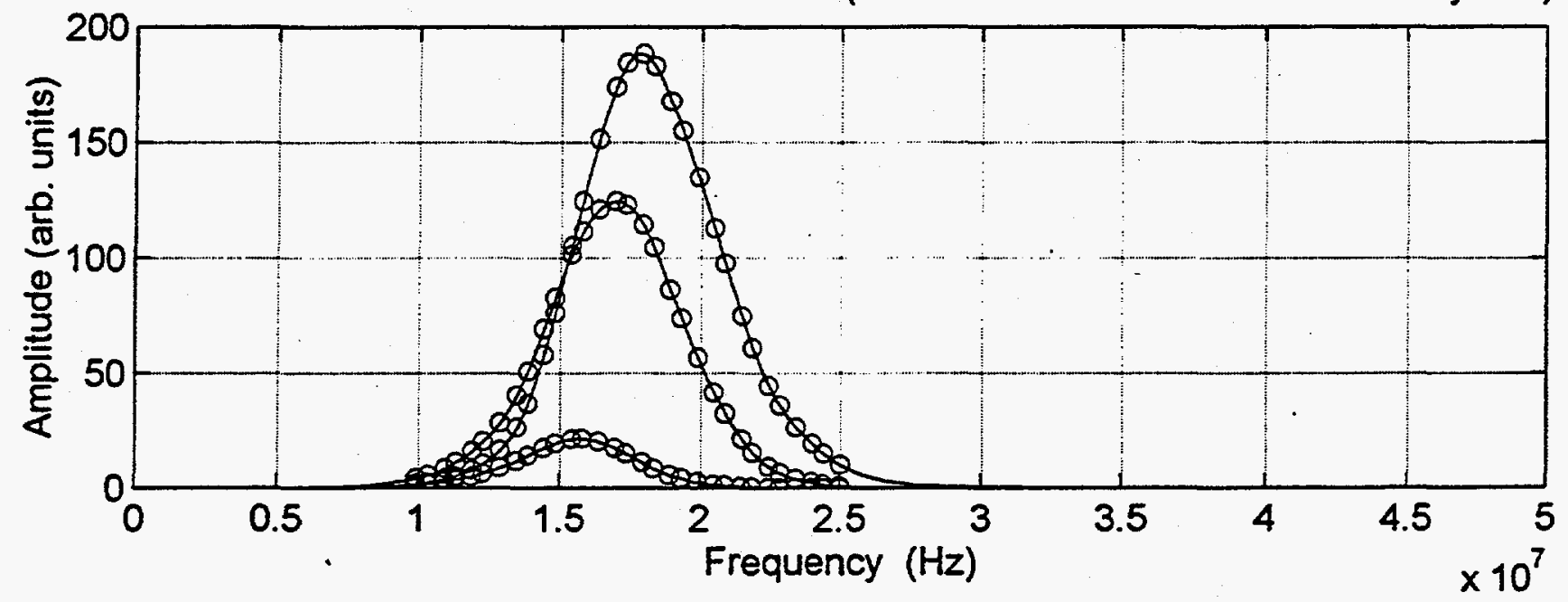

Fourier transform of wd546 wd547 wd548 ( 2 nd \& 3rd transformations scaled by 200 )

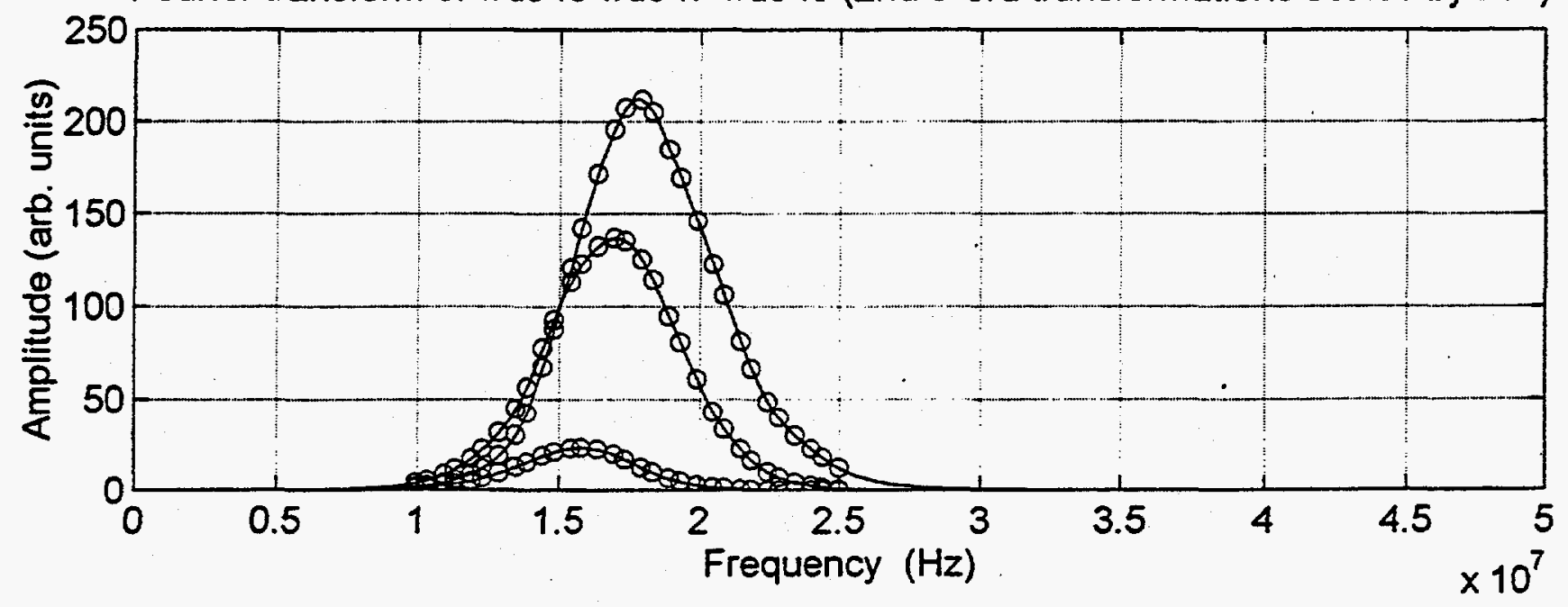




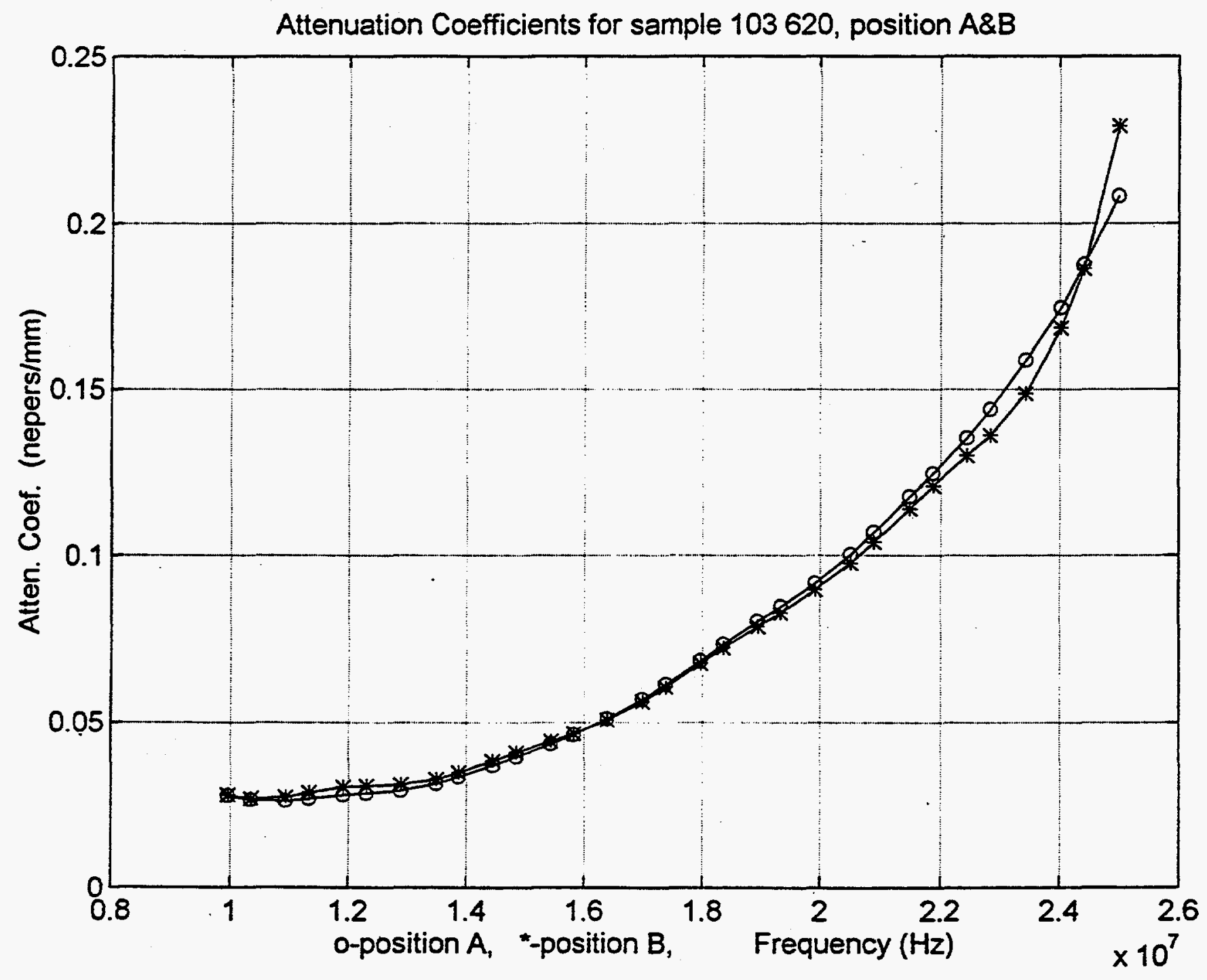



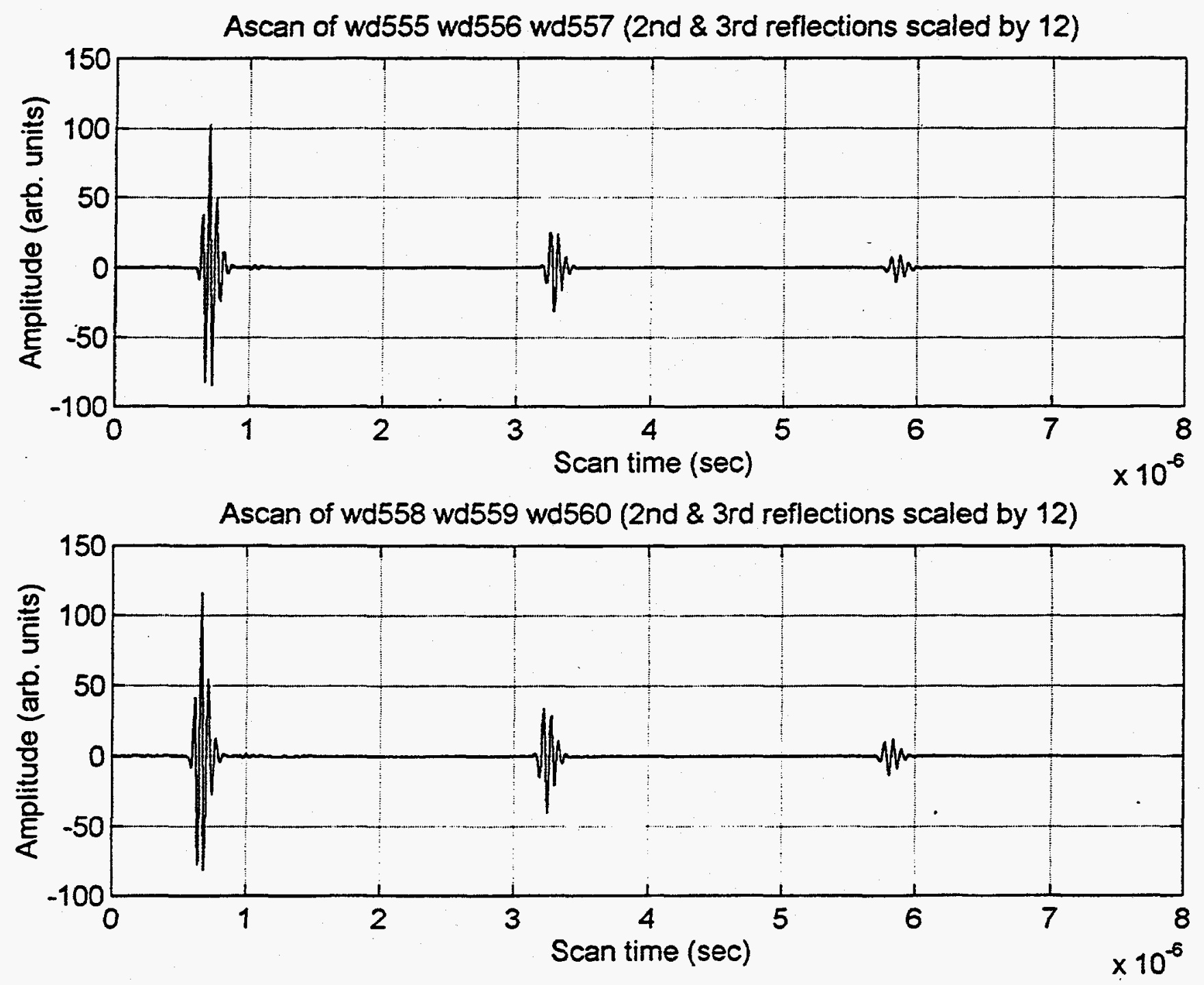
Fourier transform of wd555 wd556 wd557 ( 2 nd \& 3rd transformations scaled by 200)

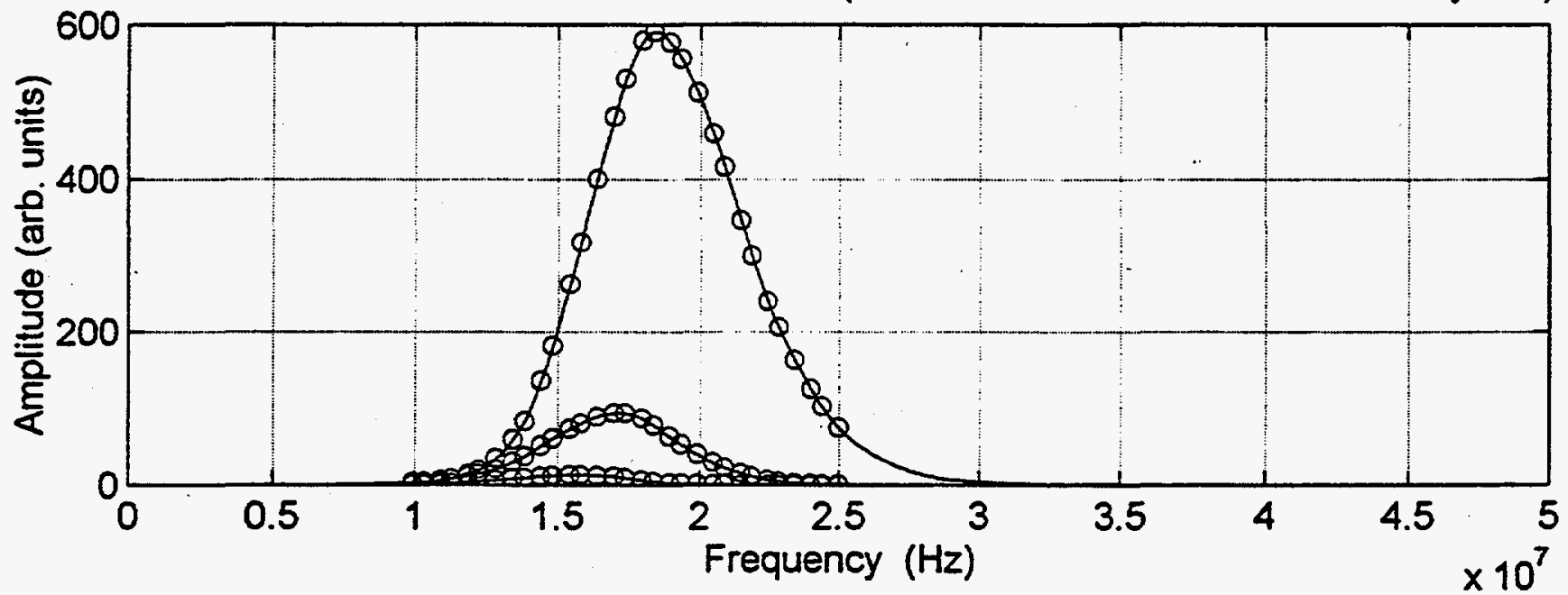

Fourier transform of wd558 wd559 wd560 ( 2 nd \& 3rd transformations scaled by 200)

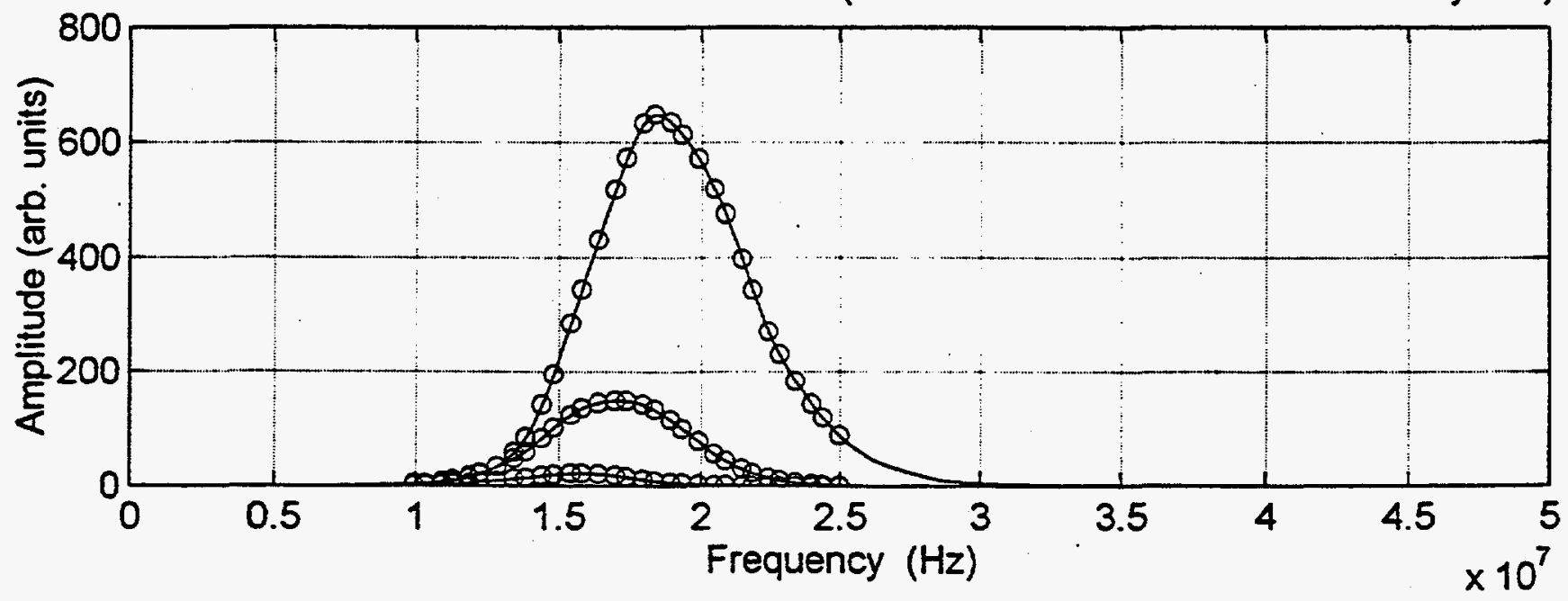




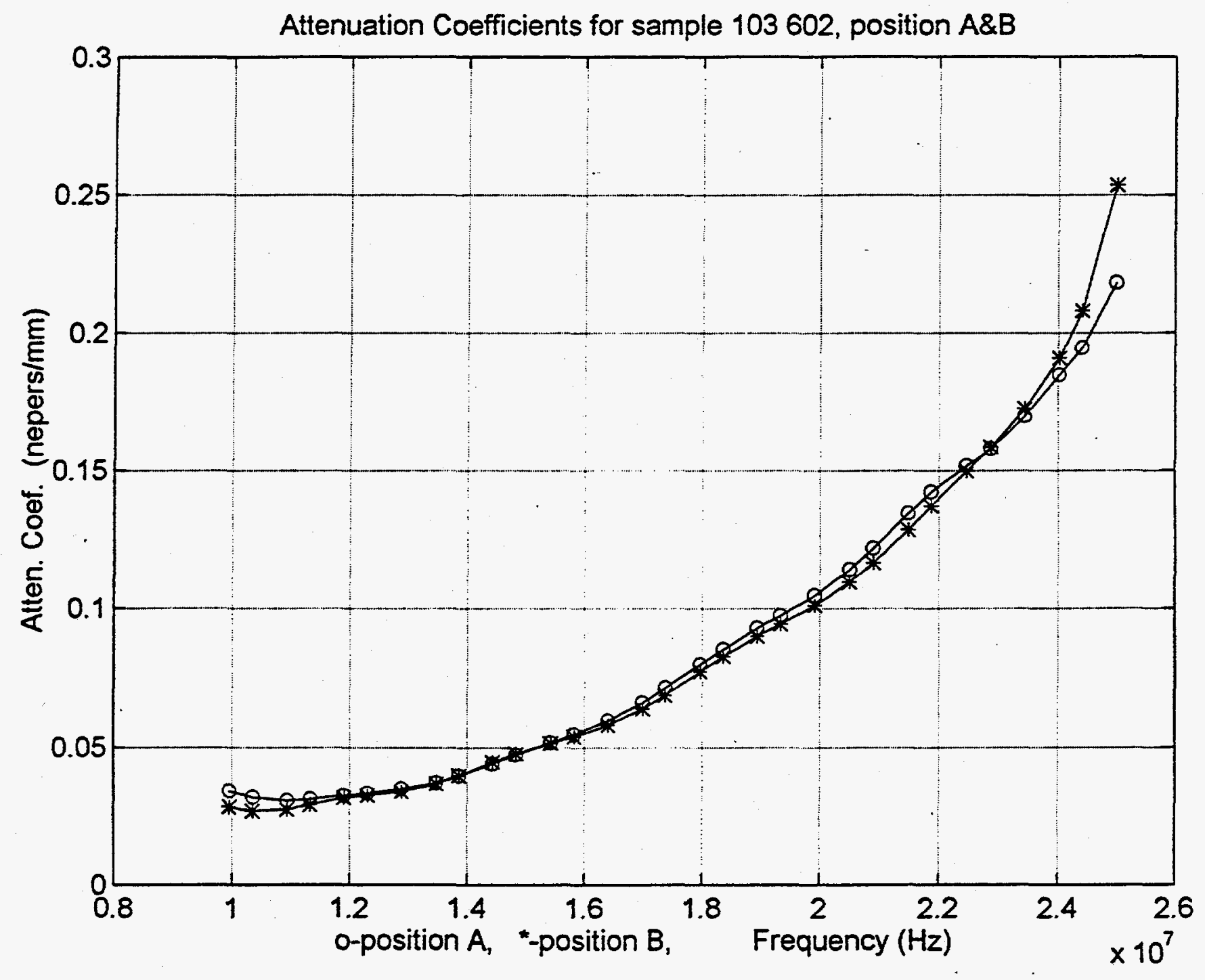



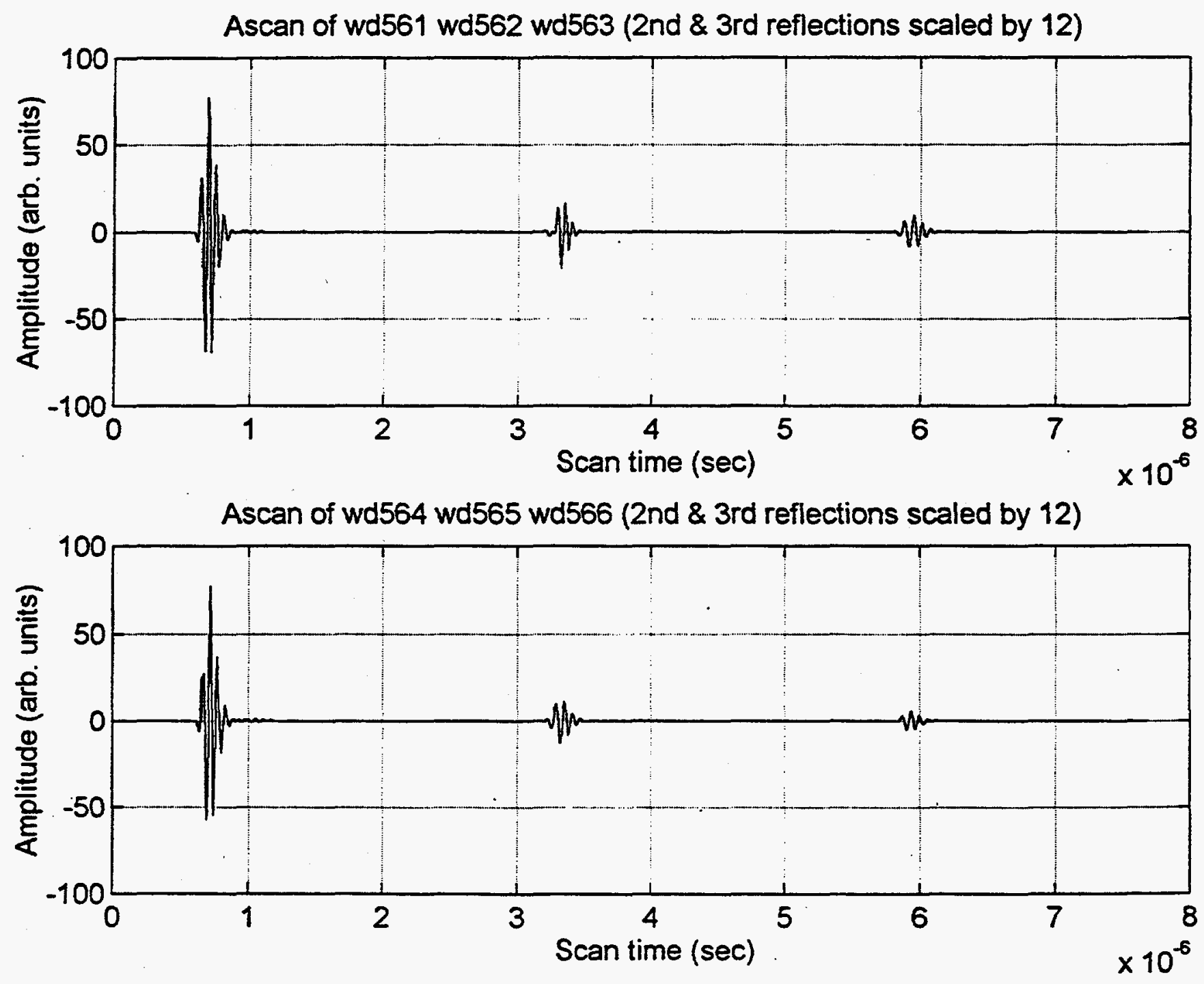
Fourier transform of wd561 wd562 wd563 (2nd \& 3rd transformations scaled by 200)

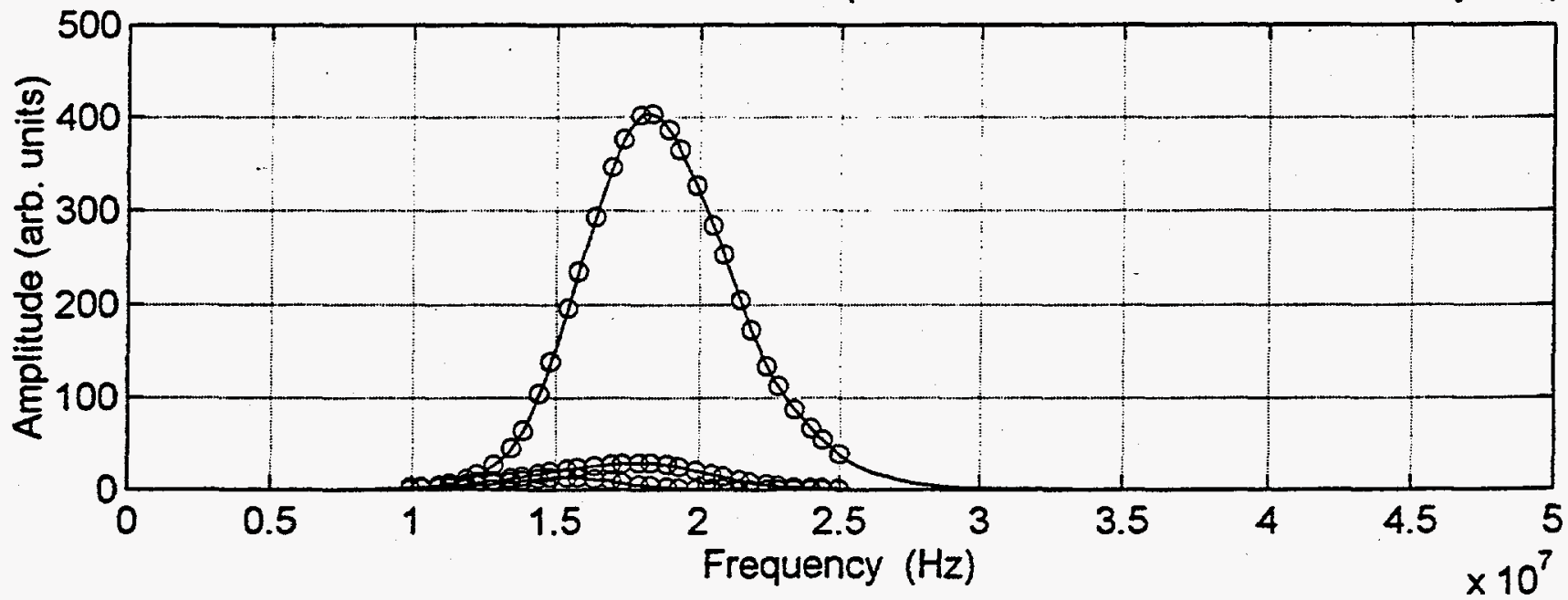

Fourier transform of wd564 wd565 wd566 (2nd \& 3rd transformations scaled by 200)

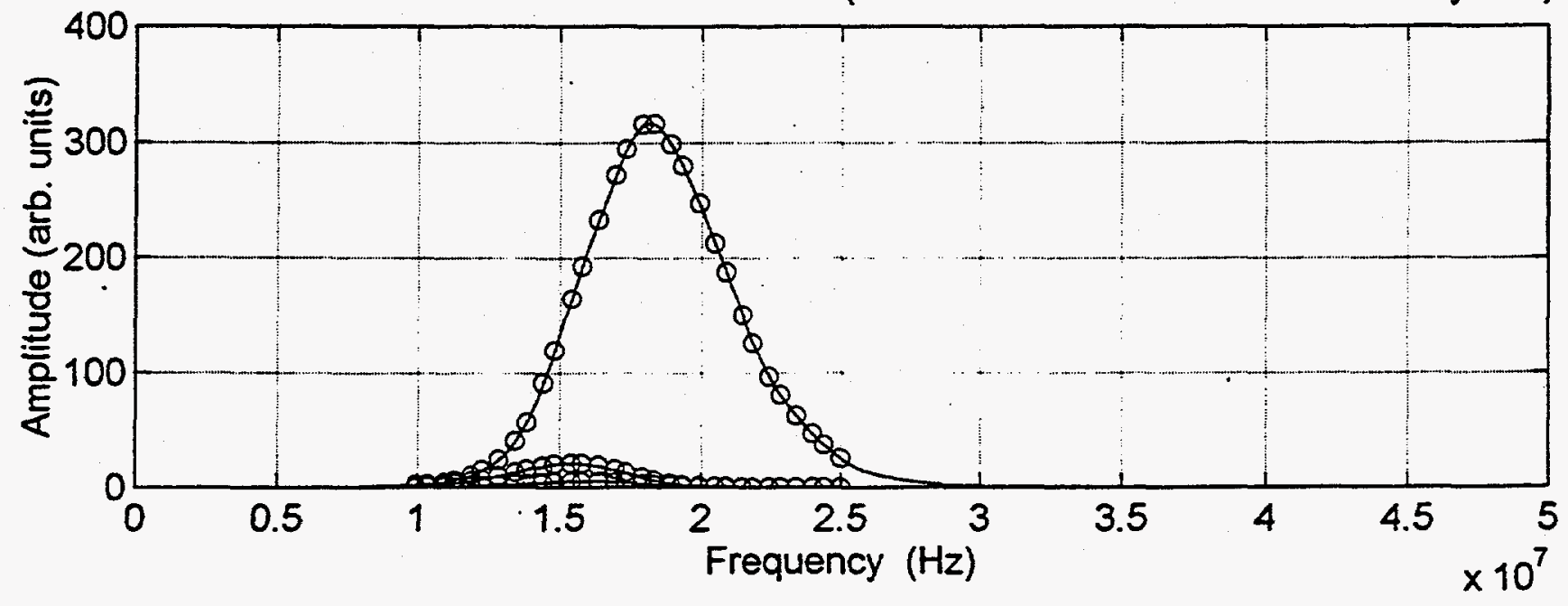




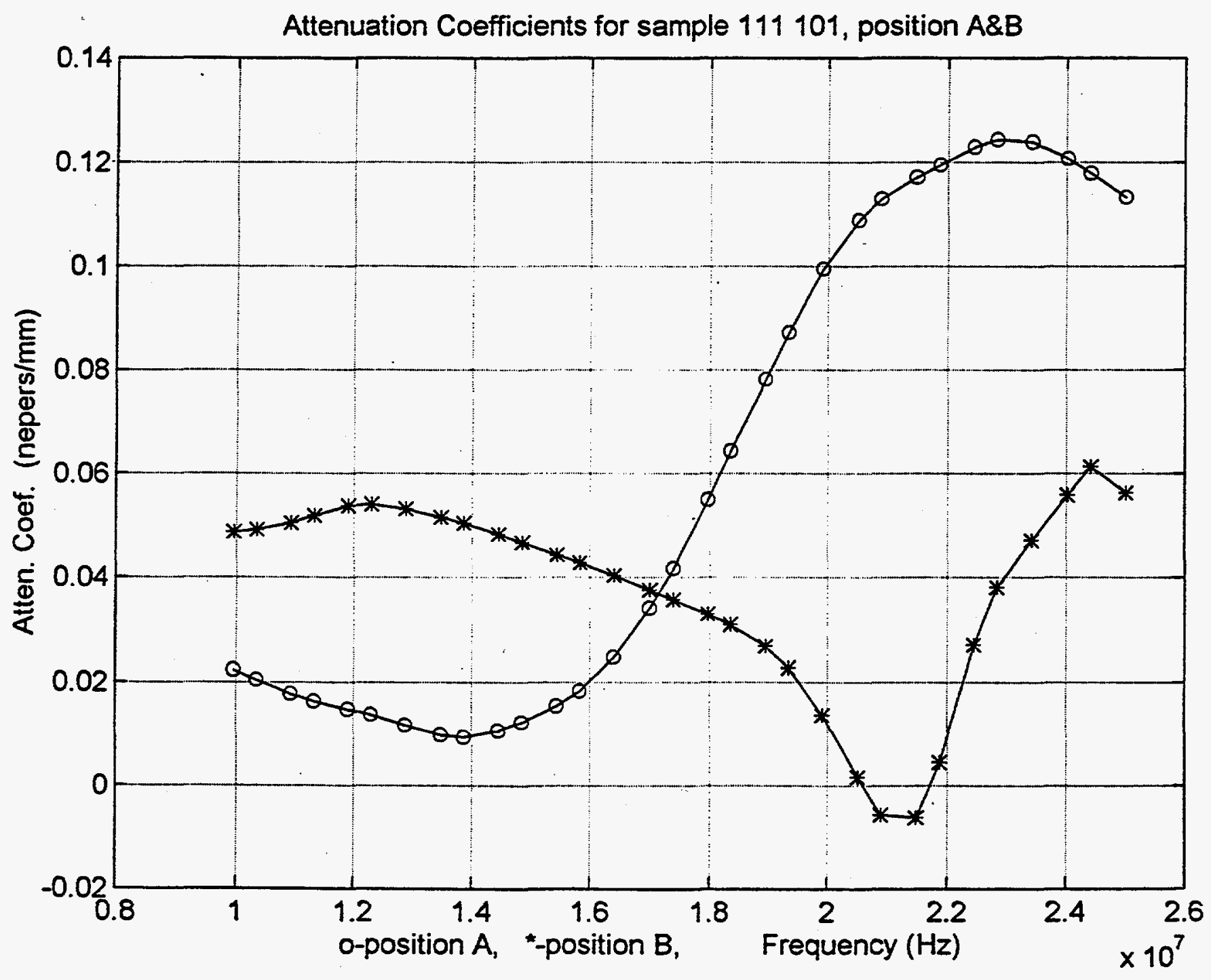



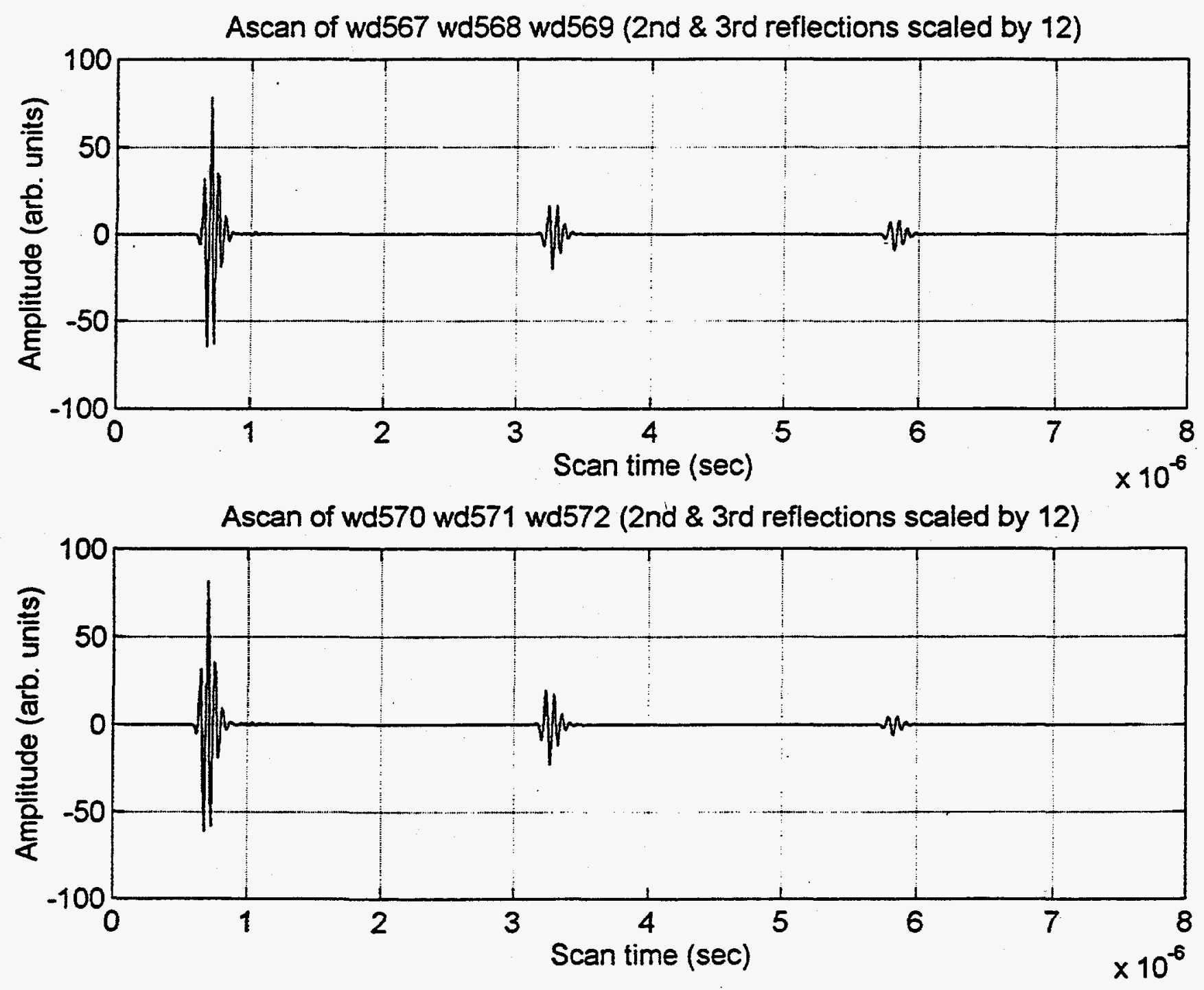
Fourier transform of wd567 wd568 wd569 (2nd \& 3rd transformations scaled by 200)

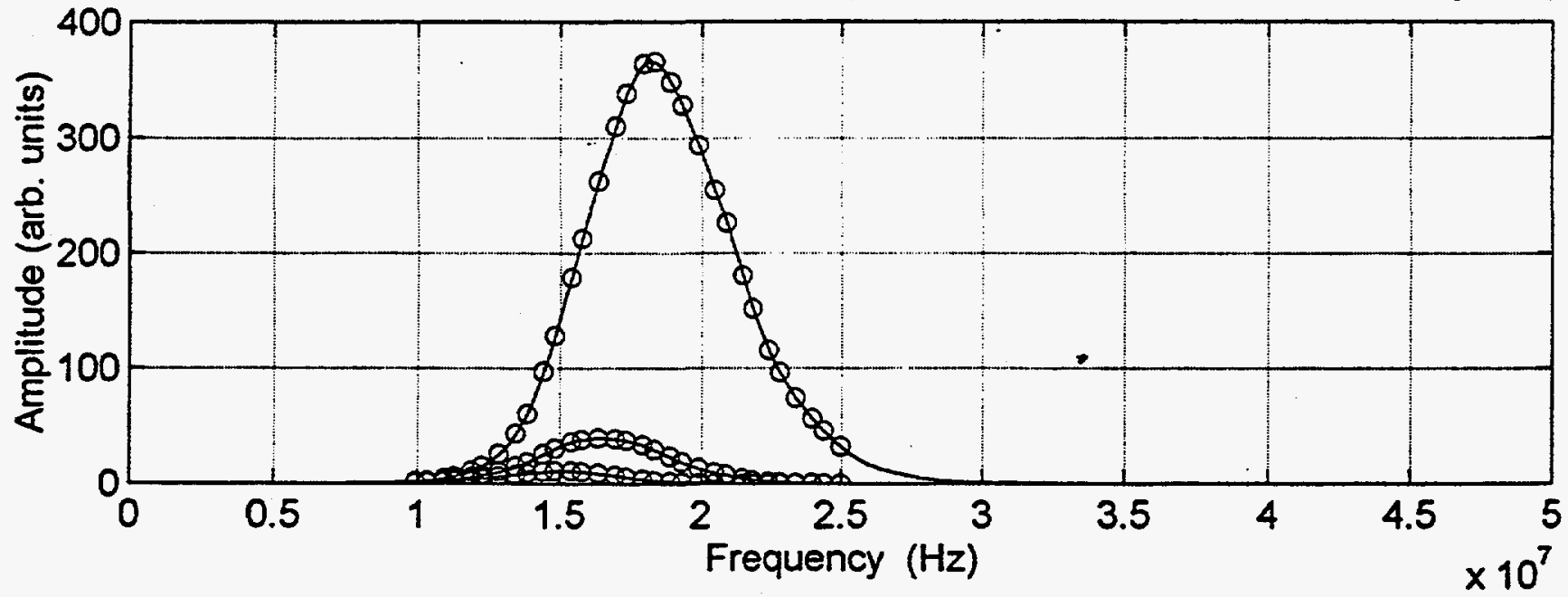

Fourier transform of wd570 wd571 wd572 (2nd \& 3rd transformations scaled by 200 )

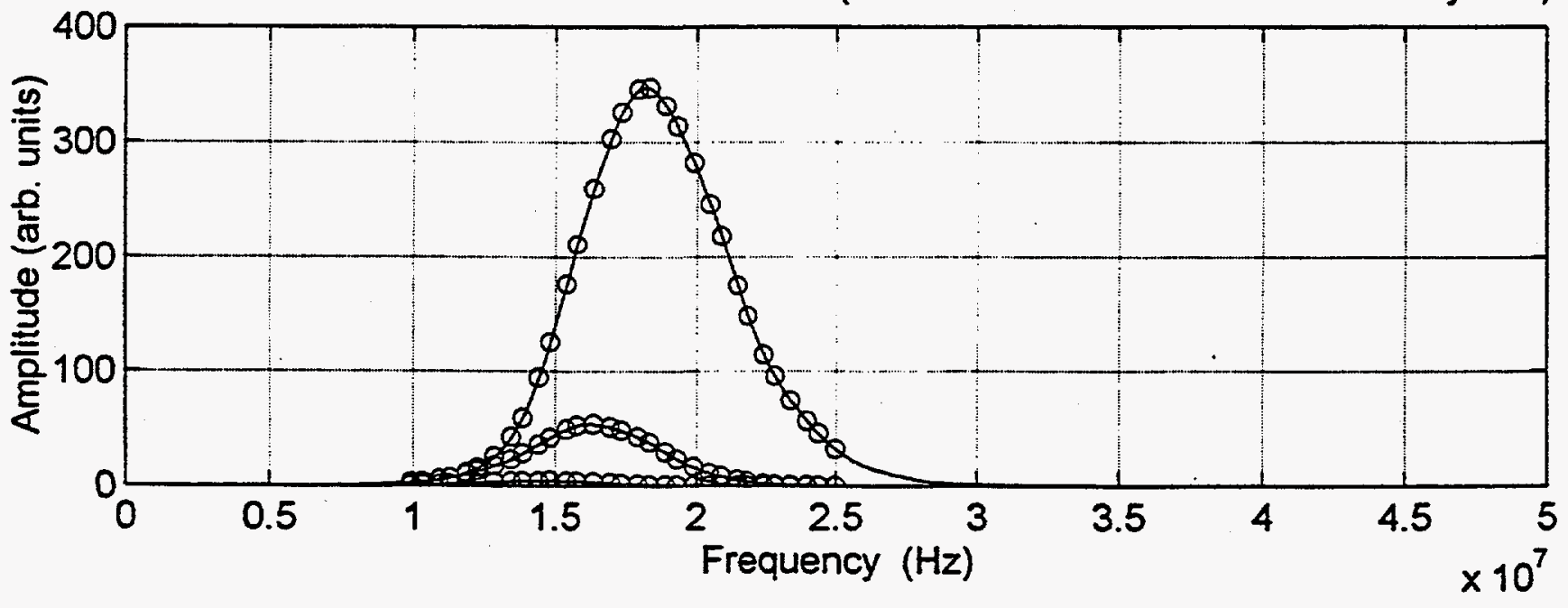




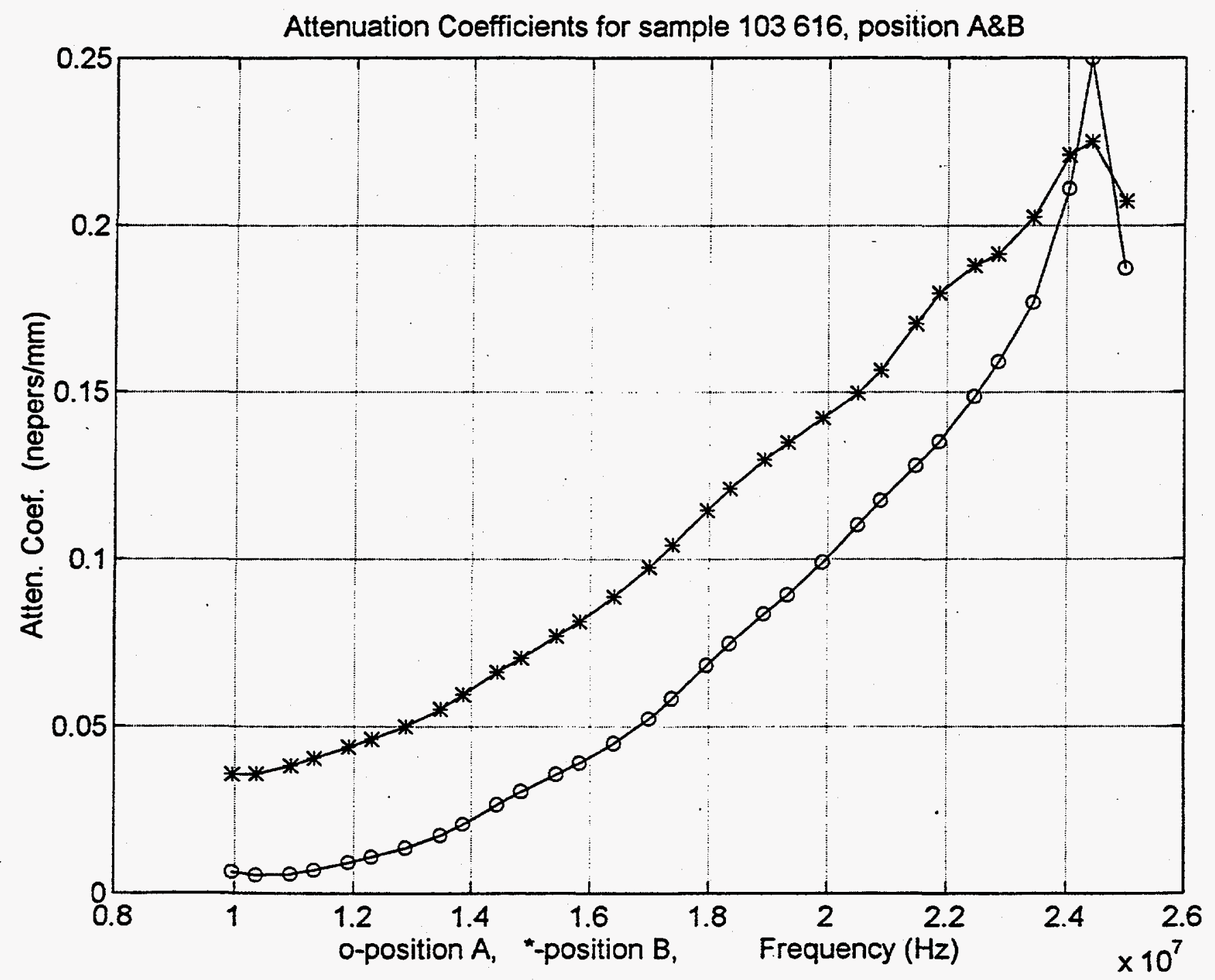



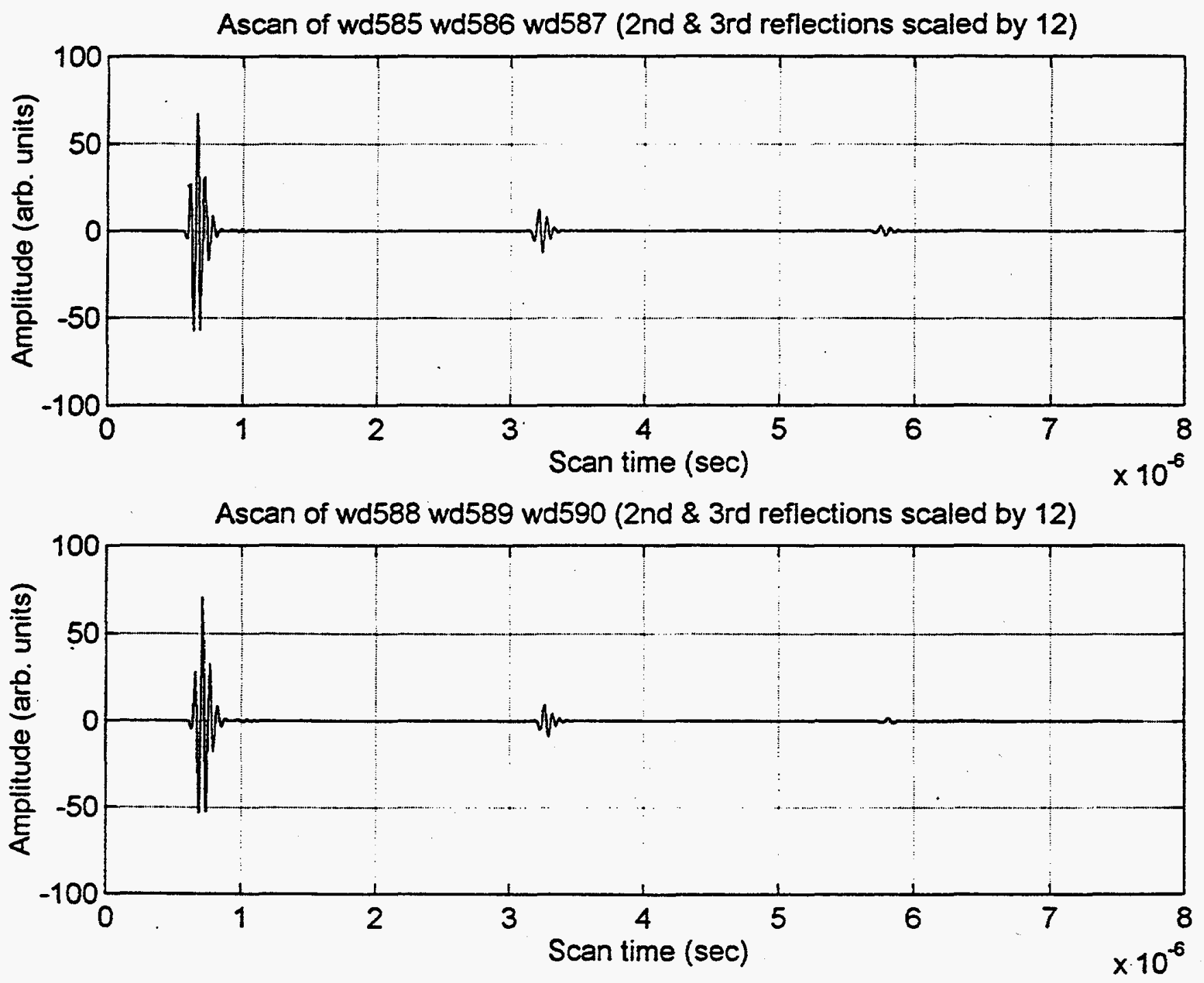
Fourier transform of wd585 wd586 wd587 ( 2 nd \& 3rd transformations scaled by 200)

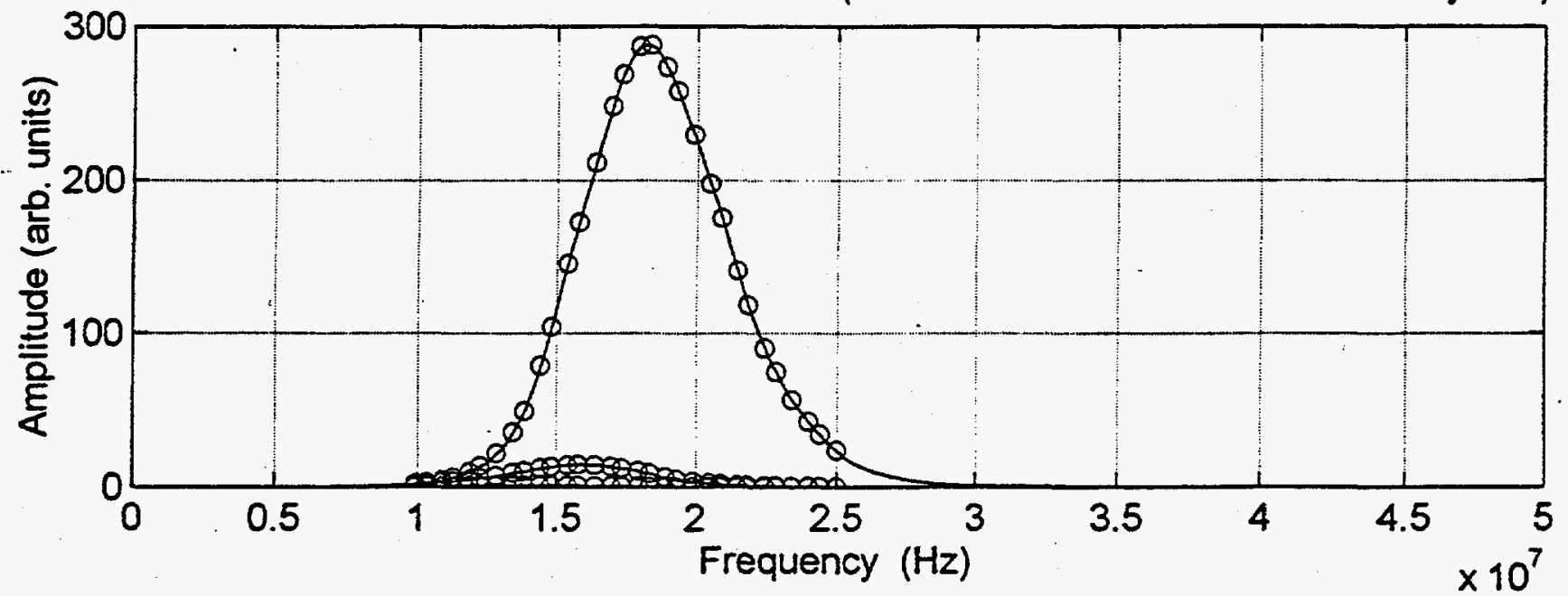

Fourier transform of wd588 wd589 wd590 ( 2 nd \& 3rd transformations scaled by 200 )

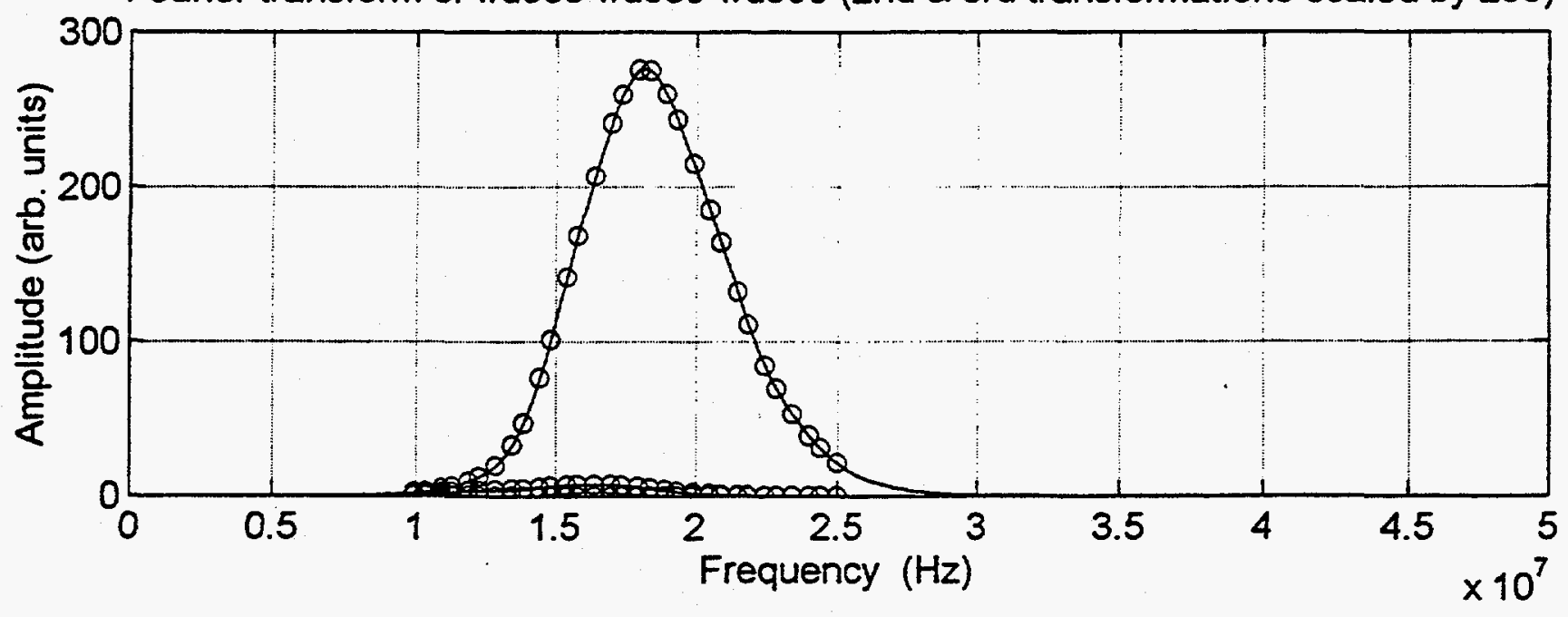




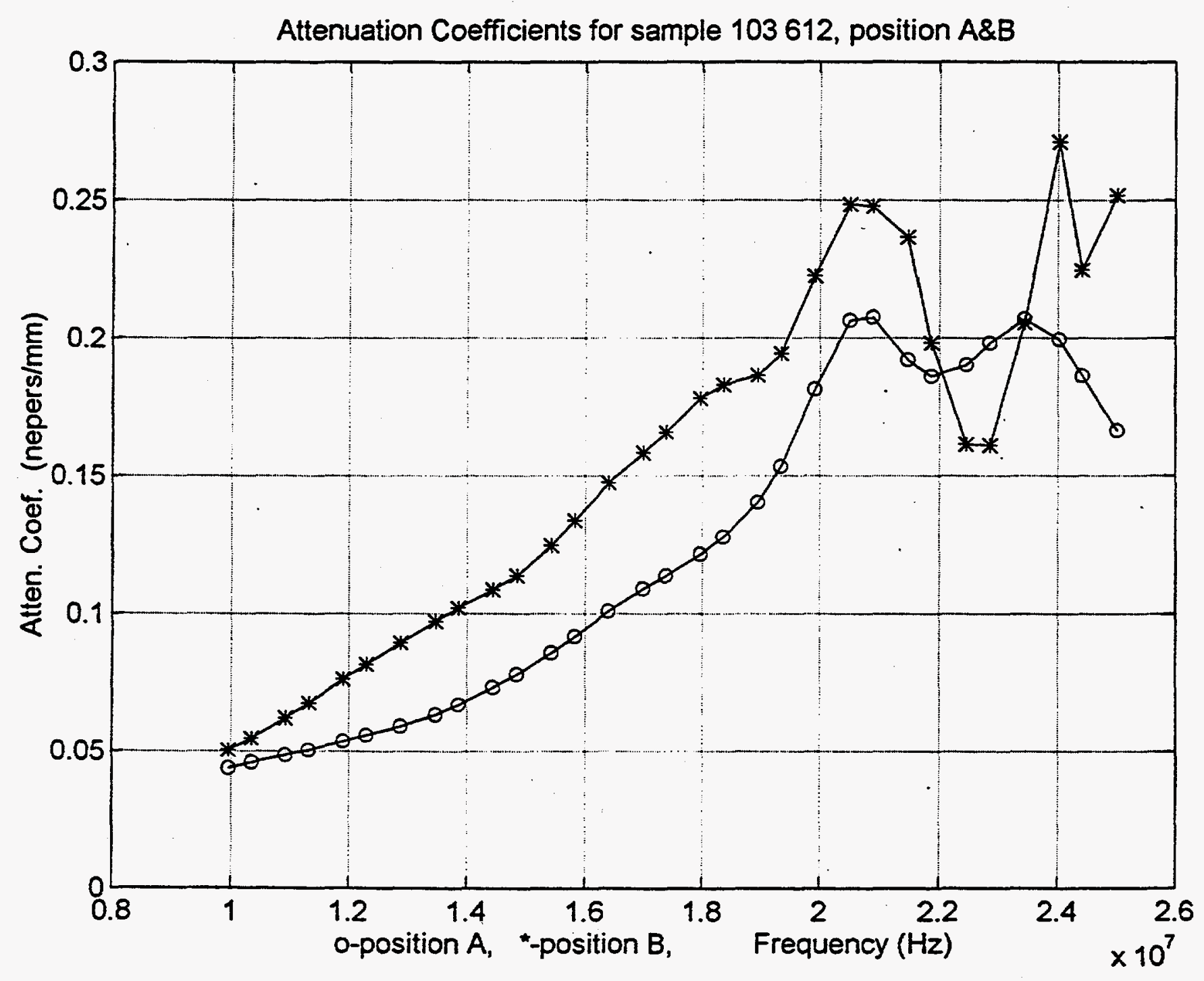



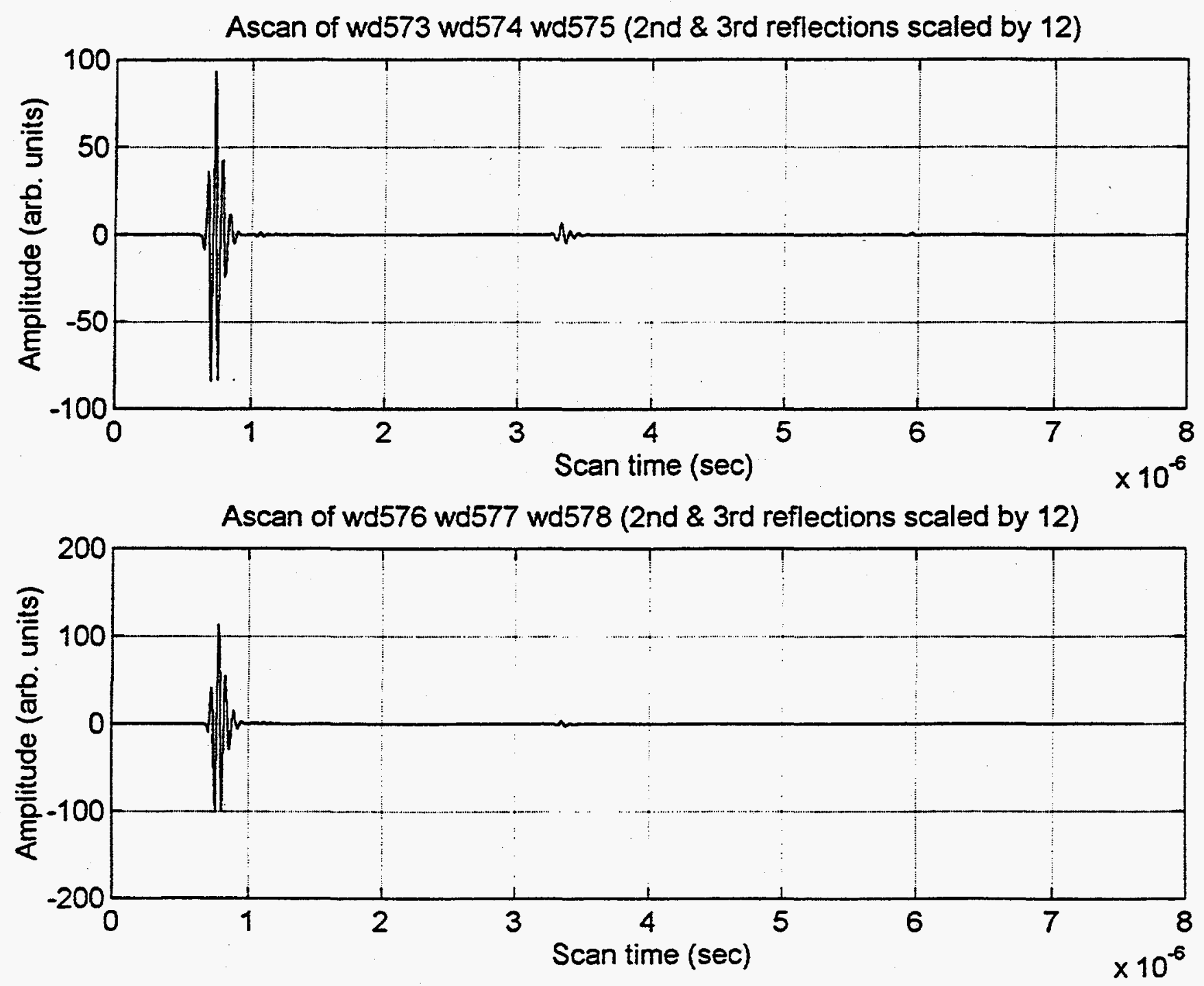
Fourier transform of wd573 wd574 wd575 ( $2 \mathrm{nd} \&$ 3rd transformations scaled by 200 )

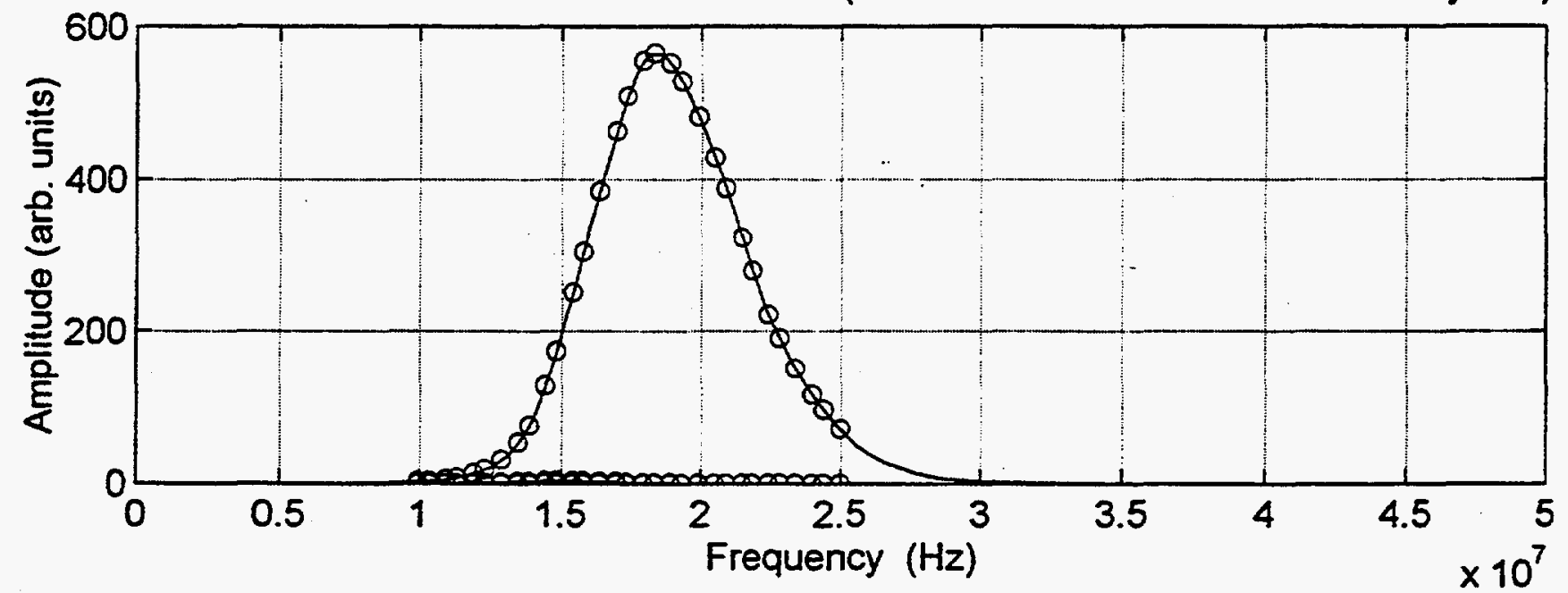

Fourier transform of wd576 wd577 wd578 ( $2 \mathrm{nd} \&$ 3rd transformations scaled by 200 )

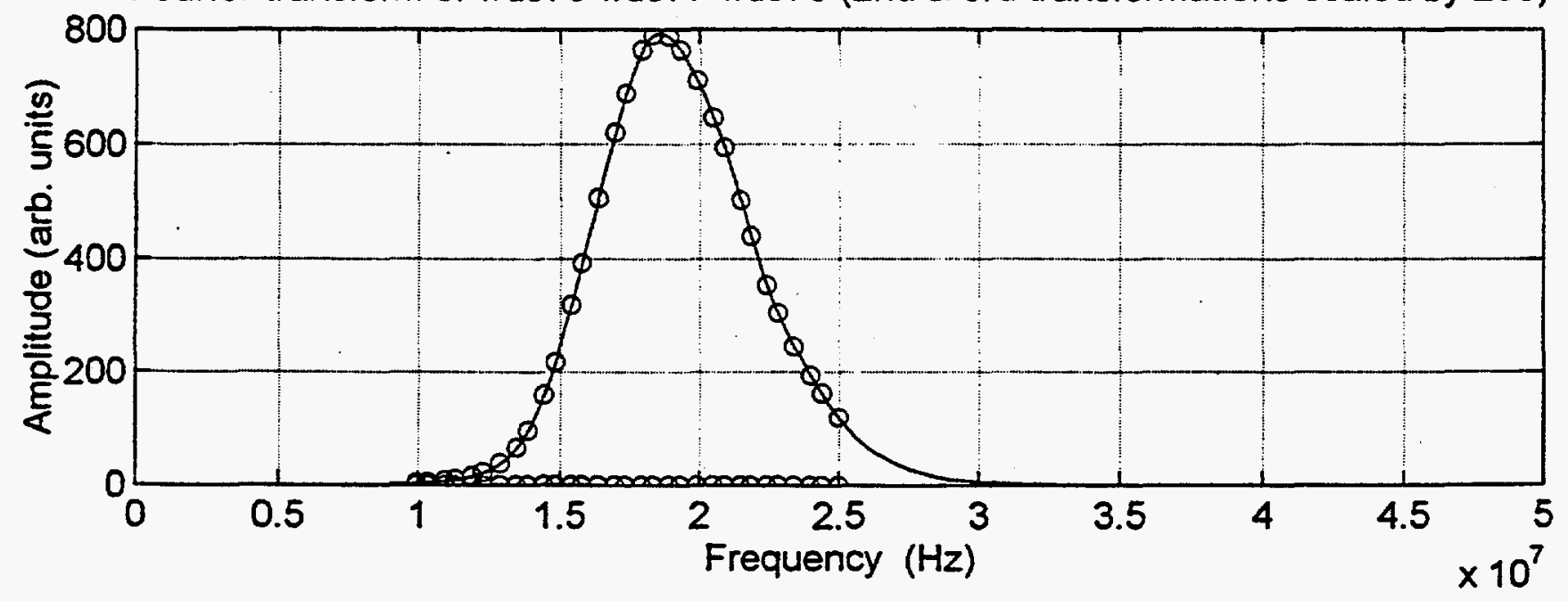




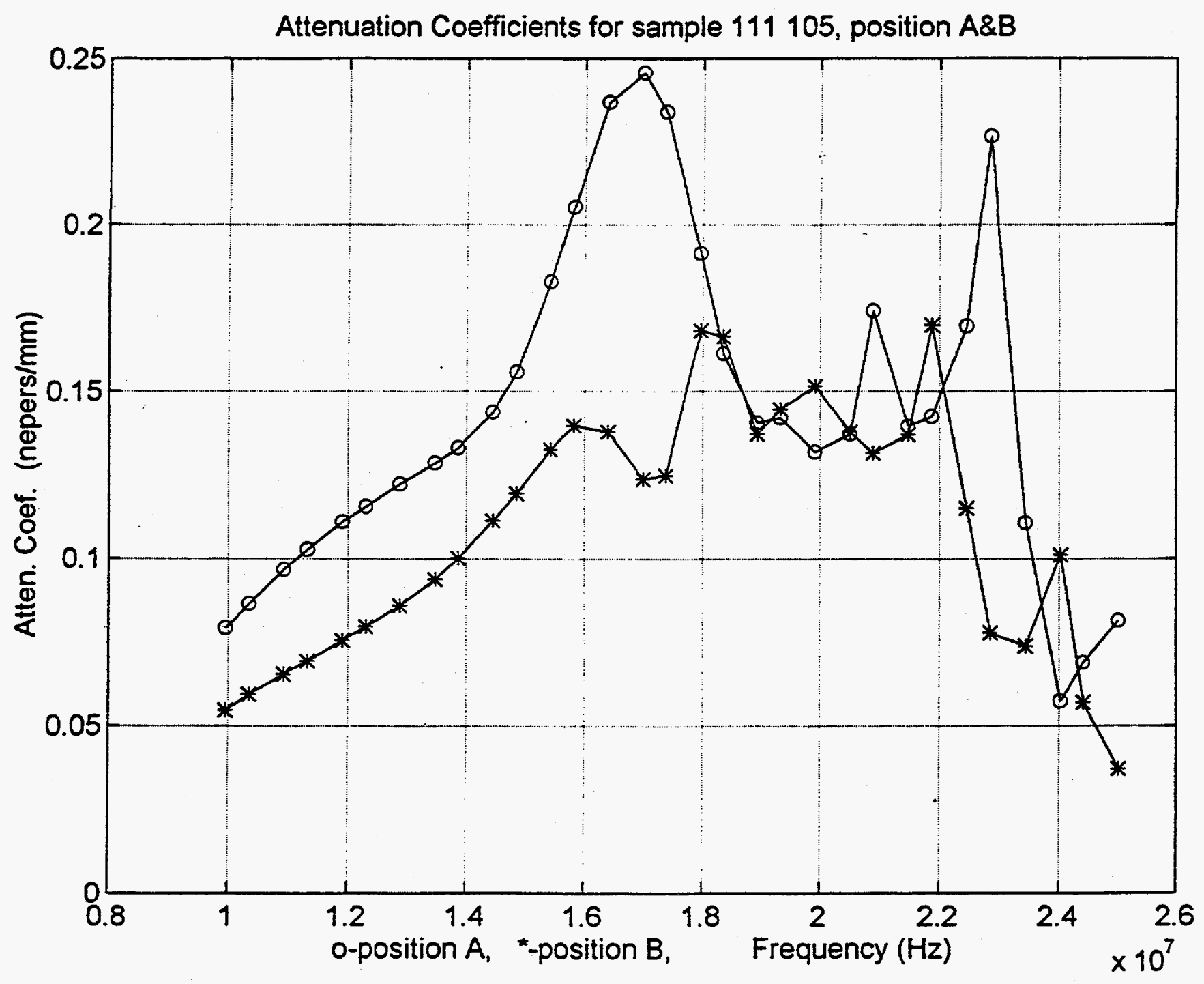



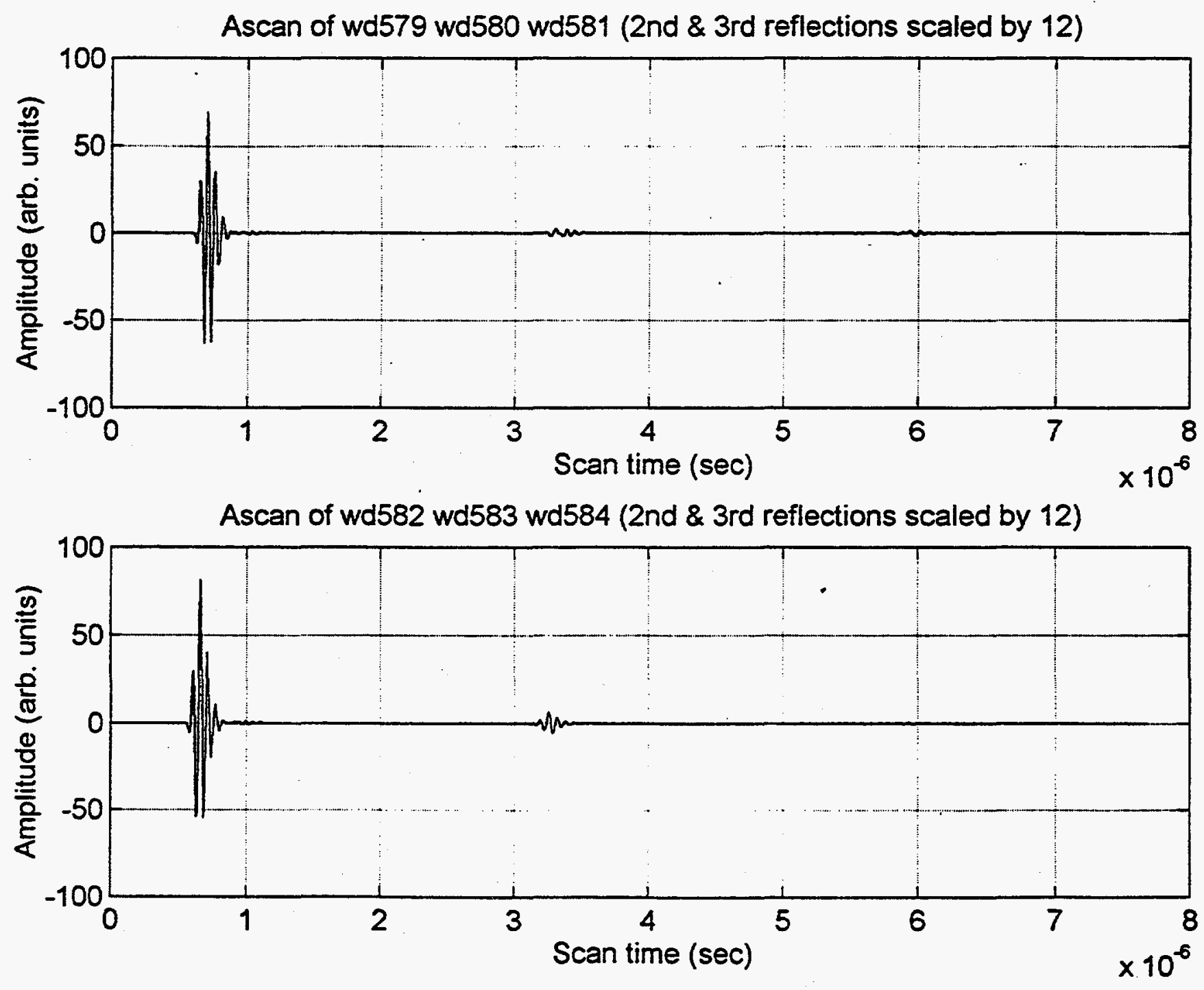
Fourier transform of wd579 wd580 wd581 ( $2 \mathrm{nd} \&$ 3rd transformations scaled by 200 )
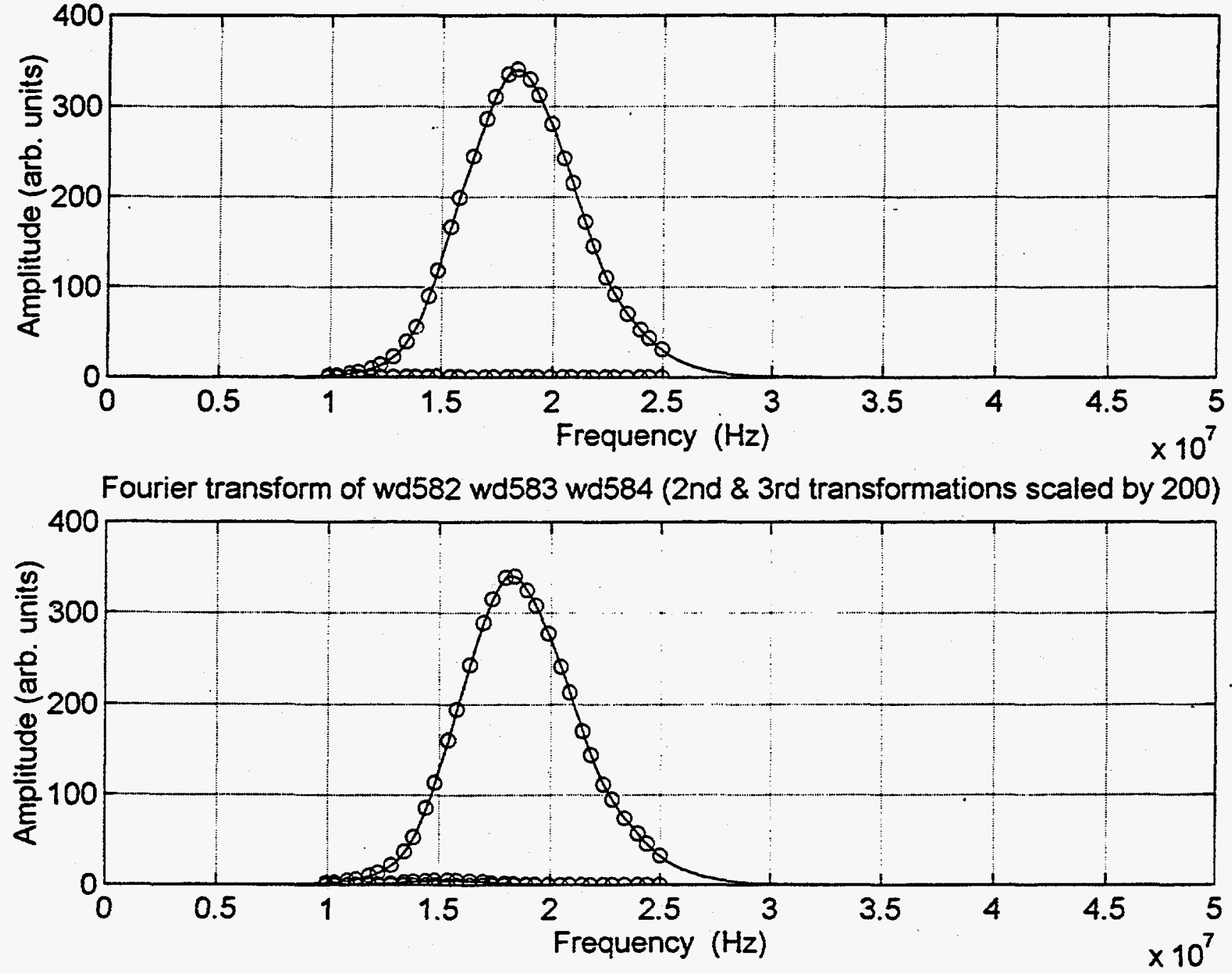


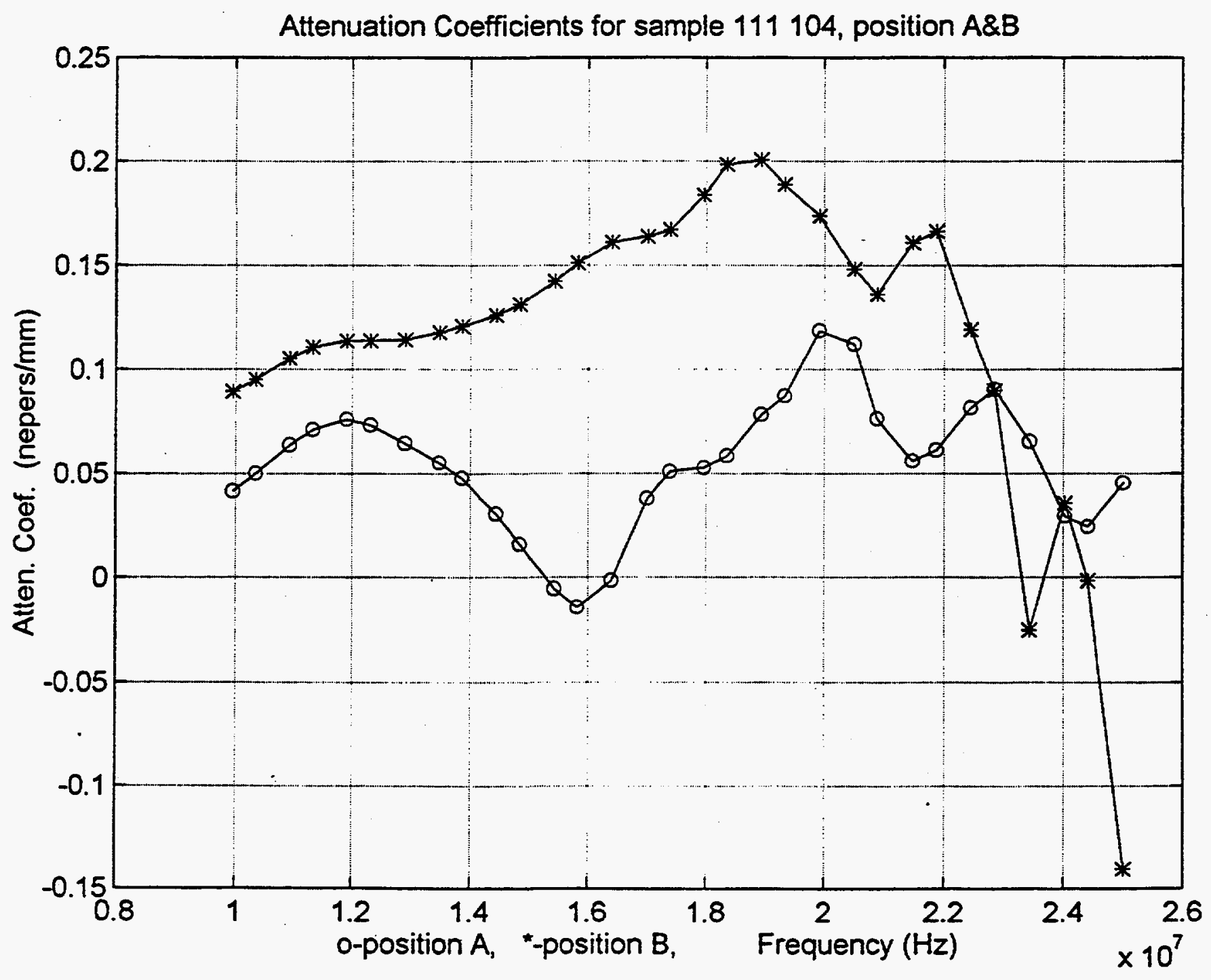




\title{
PART B: X-RAY STEREOGRAPHY TO LOCATE POROSITY ON METAL CASTINGS
}

\author{
J. Gray \\ Iowa State University \\ Center for Nondestructive Testing \\ Ames, Iowa 50011
}




\title{
FINAL REPORT ON THE EVALUATION OF X-RAY STEREOGRAPHY TO LOCATE POROSITY ON METAL CASTINGS
}

\author{
INTRODUCTION AND SUMMARY OF RESULTS
}

During the course of this work we evaluated several areas affecting the quality of cast materials. The initial objective was to identify the most promising option for work in developing $\mathrm{x}$-ray based means to improve the cost of producing quality castings. Of particular interest were methods that could be transferred to industry and that had the potential of having a real impact. There were three areas that were discussed with industries contacts at Chrysler, Harvard Industries, CMI, and Kiowa Corporation. The first area was the detection of inclusion material in rework material using energy dispersive $x$-ray techniques. The second area was the application of real time $X$-ray stereography in the inspection of castings to identify the location of porosity or inclusions relative to a surface. The third area involved coupling several emerging technologies, namely process modeling and NDE inspection modeling with solidification modeling and the efficient design of dies. The first phase of this project was to evaluate industry interest in these varied approaches and to select one for further evaluation. The activity in applying real-time stereography to locating the location of inclusions or porosity relative to the surface was selected. This was based on discussions with industrial representative including, John Balong from Kiowa, George Harmon at Chrysler, and Tom Pruka from CMI and with Dennis Kunerth at INEL. A number of samples from Kiowa and Chrysler were made available and on which work was performed. As there was significant interest in the third area, the application of NDE modeling towards optimizes inspection, a low level of activity was maintained.

The application of stereography towards locating porosity or inclusions in castings has several facets. The first is as an aid in the design of a casting die. During the design of a die the placement of gates and sprues is tested in several prototypes. The castings from these prototypes dies are often inspected by real time radiography for shrink porosity. In many cases the presence of indications is not the primary concern, rather it is their size and especially their location. The normal projection image measures the size of the porosity. The use of stereograph can reclaim the depth information lost in projection radiography. 
The point of particular interest is that this capability can be added at very low cost, especially when compared to the usual means of acquiring this depth information, namely computed tomography or destructive testing. This capability allows the rapid determination whether the location of the indication is in a critical region thus requiring another iteration on the die design.

The second area for the application of stereography is in the inspection of final product. This type of inspecting is often required in safety critical casting such as the steering linkage in automobiles. As noted before, often the issue is not whether there is an indication present, but rather where is the indication. For example, porosity that can be exposed in a machining operation can be distinguished from harmless porosity in the interior of the part. Thus acceptable parts can be certified and not thrown away and rejectable parts can be removed before the values is added. In both cases the amount of scrap generated is reduced, resulting in a lower production cost.

In this work we show that simple stereograph can achieve location accuracy of $0.2 \mathrm{~mm}$ relative to a part surface and developed an inexpensive add-on to existing real time systems thus generating an upgrade path improving the system capabilities. We have demonstrated this capability to industry representative at a workshop held in Ames and attended by 14 different local casting companies. The add-ons required to upgrade an existing x-ray real time system (RTR) depends upon the details of the system.

Assuming that any RTR system will consist of an $x$-ray generator, imaging system and a radiation, a simple joy stick sample manipulator, and a containment shield, the upgraded consists of the following: a PC for data acquisition, a precision rotation stage mounted on the sample manipulator, a calibration block, and the control Windows software. The entire upgrade package is roughly a few percent of the cost of the original RTR system.

\section{Industry Contacts}

Over the course of this project we have developed a number of industry contacts and have discussed a number of their needs. The project outlined above represents one with the highest interest. Contacts include extensive discussions with Chrysler concerning the use of models to evaluate the design of a casting. Of particular note is the use of models to evaluate the overall process. Our primary contact with Chrysler is George Harmon. 
They have indicated a willingness to supply samples and have helped in establishing contacts with two of their casting vendor, CMI Castings and Harvard industries. They have also supplied us with computer aided design CAD models of castings for us in evaluating the application of inspection models to the design of a part.

In discussions with several casting companies, especially Kiowa Corporation and Harvard Industries, two problems were identified, die design aids and identification of foreign inclusions in rework material. Of particular interest was the use of the stenography to determine the three dimensional position of porosity. The possibility of simple upgrades to existing real time $x$-ray equipment was noted. The primary contact with Kiowa is John Balong and with Harvard Industries is Don Peterson. Kiowa has indicated that they are willing to allow site trials of the stenography and are willing to supply samples. Chrysler has supplied us with several sets of samples having a range of defects over a range of severity. These sets include sand inclusions, gas bubbles, dross inclusions, and shrink porosity. These samples are being used for the stenography applications.

\section{Results of X-ray Stereography}

We developed and demonstrated an $x$-ray stereography system based on an $x$-ray image intensifier real time system. The details of stereography can be found in Appendix A, we will discuss the experimental implementation of our stenography system. The key pieces of the system consist of a rotation stage and a frame grabber capturing the RS-170 video signal. The rotational motion, the digital image data and the analysis of the data are done on the control PC. The major effort was to develop the control software and the calibration standard for the stereography system. Each of these parts of the system will be discussed.

The need for a calibration standard comes from the requirement that in the stereography calculations the laboratory coordinate system must be precisely known. Specifically, the source to sample to detector distance must be known to millimeter accuracy in order to obtain the $0.2 \mathrm{~mm}$ positional information. A calibration standard was made of a machinable tungsten alloy with dimensions of 2 in. $\times 1$ in. $\times 0.25$ in. The standard is used to calibrate the measurement system. The block is placed on the image intensifier and is used to calculate the size of the pixels. The precise knowledge of the block size and the $x$-ray opaque nature of the material make this a simple process. The block is then placed on the sample manipulator and positioned so the that image is 2 
times the original size. The amount of motion required to generate a $3 \mathrm{X}$ image is measured, either by the linear motion control or by a caliper. The source to detector distance is 6 times that measured distance, see Figure 1.

At $3 \mathrm{X} \mathrm{a}^{\prime}=\mathrm{D} / 3$ and at $2 \mathrm{X} \mathrm{a}=\mathrm{D} / 2$, so the difference $\mathrm{a}^{\prime}-\mathrm{a}=\mathrm{D} / 6$.

The data acquisition is done via a Windows interface. The procedure consists of placing a small lead tag on the surface of the casting, usually on the surface that is of concern, this surface can be on exposed by machining. The part is then examined in a calibrated RTR system. The first projection is viewed by the user and point on the image are collected via mouse clicks, in this case the surface tag and the internal porosity. The part is rotated by means of the precision rotation stage, usually 30 degrees. The points from the surface tag and the porosity are again identified on the rotated image. The change on the relative pixel distances between the two points of interest together with the rotation angle and the system calibration give the information required to calculate the $x, y, z$ coordinates of the two points. From this the distance of the porosity from the surface can be calculated. A more detailed description of the procedure is included in Appendix B. This is an excerpt from G. Nutakki MS thesis. This thesis is under preparation and the complete version should be available in several months. A portion of this thesis contains a description of the work on developing the $\mathrm{x}$-ray real time stereography data acquisition system.

The results generated from this system allow a user to measure the location of porosity to with in $0.2 \mathrm{~mm}$ of the surface. The time for this measurement is principally governed by the users selection of the initial points and the rotation of the part. The calculation of the porosity coordinates takes a few milliseconds.

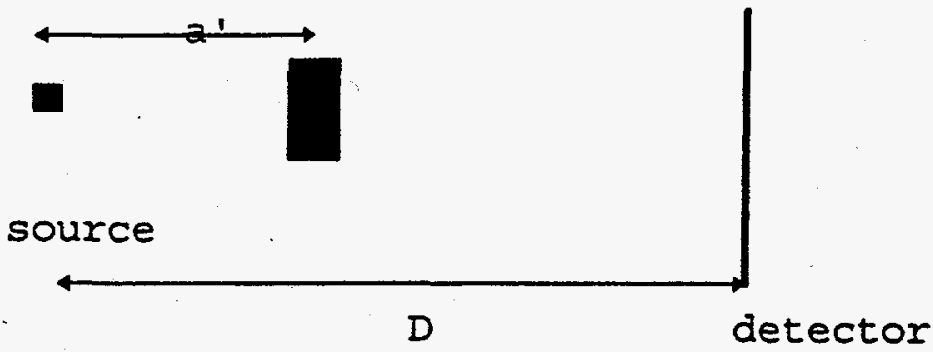

Figure 1. Source to sample to detector distances. 
The system was demonstrated to representatives of 14 Iowa casting cōmpanies at a workshop on Advanced radiography Methods for Inspection Castings held at Iowa State University. This workshop was held in conjunction with the Iowa Demonstration Laboratory. 
APPENDIX A

3-D FLAW RECONSTRUCTION 


\section{APPENDIX A \\ 3-D FLAW RECONSTRUCTION}

This Appendix describes the mathematical foundation used to reconstruct the three dimensional coordinates of flaws and other features of interest within a host material. This method is restricted to determining point locations as opposed to computed tomography methods which reconstruct a map of the entire X-ray attenuation of a sample cross-section. Determining the coordinates of only the points of interest is much more computationally efficient, and often provides sufficient information for an NDE practitioner to make a decision.

The point reconstruction method developed for this work is called the generalized stereography (GS) method. It closely resembles the work of Wallingford (1990); the main differences are the generalized approach for sample manipulation and the elimination of nuisance parameters in the system inversion. Wallingford's work as well as other well known methods for determining flaw depth are briefly described prior to deriving the GS equations.

The Appendix concludes by describing how to incorporate the GS equations into a real-time radiography system. A simulation of the measurement process (forward model) and the solution process (inverse model) is used to gain insight into the error sensitivity of the system under a variety of operational configurations.

\section{Existing Methods}

Stereoradiography (Quinn and Sigl, 1980) is a qualitative technique based on the same principle as a stereoscopic photograph viewer. Two radiographs of the sample are required. Between exposures, the $\mathrm{X}$-ray source is shifted a distance equal to the spacing of the human eyes. These radiographs are placed in a stereoscopic viewer, and the operator perceives the depth of flaws within the sample. Perceiving the orientation of the feature within the part is aided by placing a uniform grid of lead wires around the sample or by painting lead stripes on the sample (Veress, 1989).

Stereo X-ray photogrammetry (Veress, 1989) is a quantitative version of stereoradiography. $\mathrm{X}$-ray photogrammetry is based on a central projection model which assumes a point $\mathrm{X}$-ray source and straight line propagation of the $\mathrm{X}$-rays. The latter condition holds due to the nature of 
electromagnetic wave propagation. The former condition of a point source is not realized in an actual $\mathrm{X}$-ray source, and the ensuing geometric unsharpness makes feature identification more ambiguous. The microfocus source, however, is an extremely good approximation to an ideal point source, so unsharpness problems are minimized. The straight line propagation of the $\mathrm{X}$-ray path implies a collinear relationship between the X-ray source feature point, and detector points. McNeil (1966) identifies four types of X-ray photogrammetric lab setups: (1) two stationary sources with a stationary object, (2) translation of a single source with stationary object, (3) stationary source with object translation, and (4) stationary source with object rotation. In all cases the object is kept in contact with the X-ray film detector. Case 4 is preferred since equipment complexity is minimized. Case (3) requires a significant amount of translation, so the X-ray source must be rotated between views. X-ray photogrammetric techniques emphasize system calibration as a means to achieve low measurement errors, e.g., $\pm 0.004 \mathrm{~cm}$ in depth (Veress, Lippert, and Takamoto, 1977).

The parallax method (Bryant and McIntire, 1985) uses source shifting and similar triangle relationships to quantitatively determine flaw depth within a sample. Figure A.1 shows a diagram of the parallax radiography setup. The X-ray source is translated a known distance $A$ in a line parallel to the X-ray detector. This produces a corresponding shift $B$ in the image of the flaw on the film. The objective is to determine the flaw distance $\mathrm{D}$ above the detector plane. Knowing the source-to-detector distance $S$, the rule of similar triangles implies

$$
\frac{B}{D}=\frac{A}{S-D}
$$

Solving for D yields

$$
D=\frac{S B}{A+B}
$$




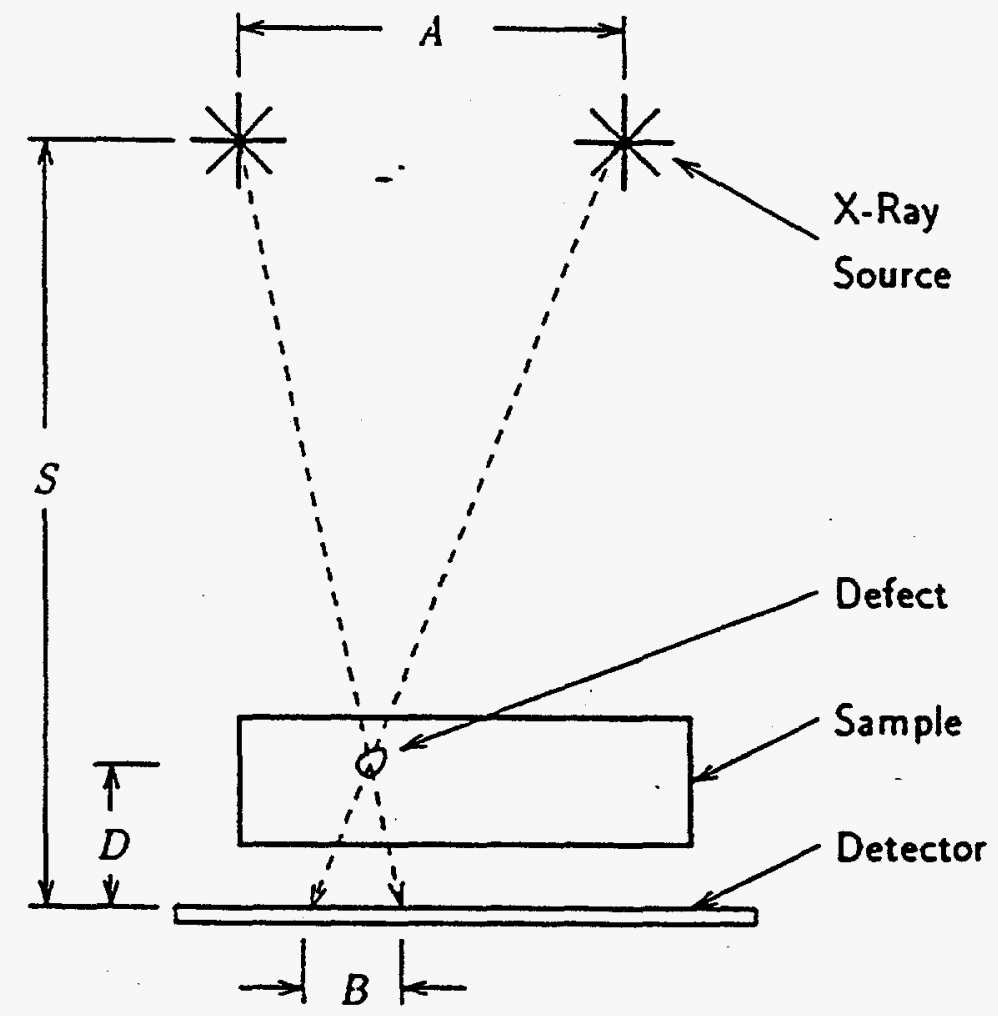

Figure A.1. Parallax method for measuring depth of a flaw in a material.

This technique requires the source shift and source/detector distances to be known accurately. Furthermore, since the result of the method is the flaw height above the detector, the sample thickness and position relative to the detector must be accurately known as well to properly locate the flaw relative to the boundaries of the sample. This problem is alleviated by applying lead markers on the source side and detector side of the sample. The formula of Equation (A.2) is applied for both markers. This determines the boundaries of the sample relative to the detector, so knowledge of the sample thickness is not required.

Stereography (Wallingford 1990) is a method for reconstructing the three dimensional coordinates of feature points within a sample. This approach uses a sample shift rather than a source shift. The method was implemented using both X-ray film and the real-time detector. A pair of images is collected and input to an off-line program to compute the feature depth. 
The X-ray source position is indicated by the vector $S=S_{x} i+S_{x} j+S_{z} k$. The ray connecting the source, the point of interest $P$, and the measurement point $M$ in the detector plane is described by the parametric equation $R(t)=S(l-t)+M(t)$. As $t$ varies from 0 to $l, R(t)$ traces out the ray from the source to the measurement point. The vector $R(t)$ coincides with $P$ at some value of $t=t_{1}$. The sample is shifted an amount $T$ in a line parallel to the $X$-ray detector plane. This translates the point of interest to a new position $\mathbf{P}^{\prime}$ which projects a new measurement point at $\mathbf{M}^{\prime}$. The new ray selected by $S, P^{\prime}$, and $M^{\prime}$ is described by a second equation $R^{\prime}(t)$. It coincides with $P^{\prime}$ at $t=t_{2}$. These two measurements yield a system of equations which is inverted to solve for the unknown values $P_{x} P_{y}, P_{z}$, and the "nuisance parameters" $t_{1}$ and $t_{z}$.

Wallingford demonstrated that this technique could easily accommodate additional measurements to produce a least-squares estimate of the point position P. Furthermore, he derived an extension of the method which could accommodate sample rotation as well as translation. In the subsequent derivation, the sample manipulation is generalized using a linear transformation of the form $\mathrm{y}=\mathrm{Ax}+\mathrm{b}$. This transformation permits the sample to be manipulated in a more complex manner than simple translation.

The main drawback of the parametric approach is that a new parameter $t_{i}$ is introduced for each new measurement. The point of interest $P$ and its translates $P_{i}$ are functions of these parameters, so there is redundancy in this system formulation. In the following derivation, the fact that $S, P$, and $\mathrm{M}$ are collinear is expressed using a cross product approach rather than a parametric equation approach. This non-parametric method is free of nuisance parameters.

\section{Mathematical Formulation}

The following derivation is designed for a fixed-position $\mathrm{X}$-ray source, a fixed position X-ray detector, and a movable sample. This is a limitation of the particular RTR laboratory used for this work (i.e., the X-ray source and detector positions are not computer controlled). However, this method can easily be reformulated in terms of a movable source and a fixed sample.

Figure A.2 shows a diagram of the experiment geometry. The $\mathrm{X}$-ray source location is denoted by the $3 \times 1$ vector $s=\left[s_{1} s_{2} s_{3}\right]^{T}$ (the vector indices 1,2 , and 3 correspond to the $X, Y$, and $\mathrm{Z}$ axes, respectively, of the global coordinate system; this convention is used throughout the 


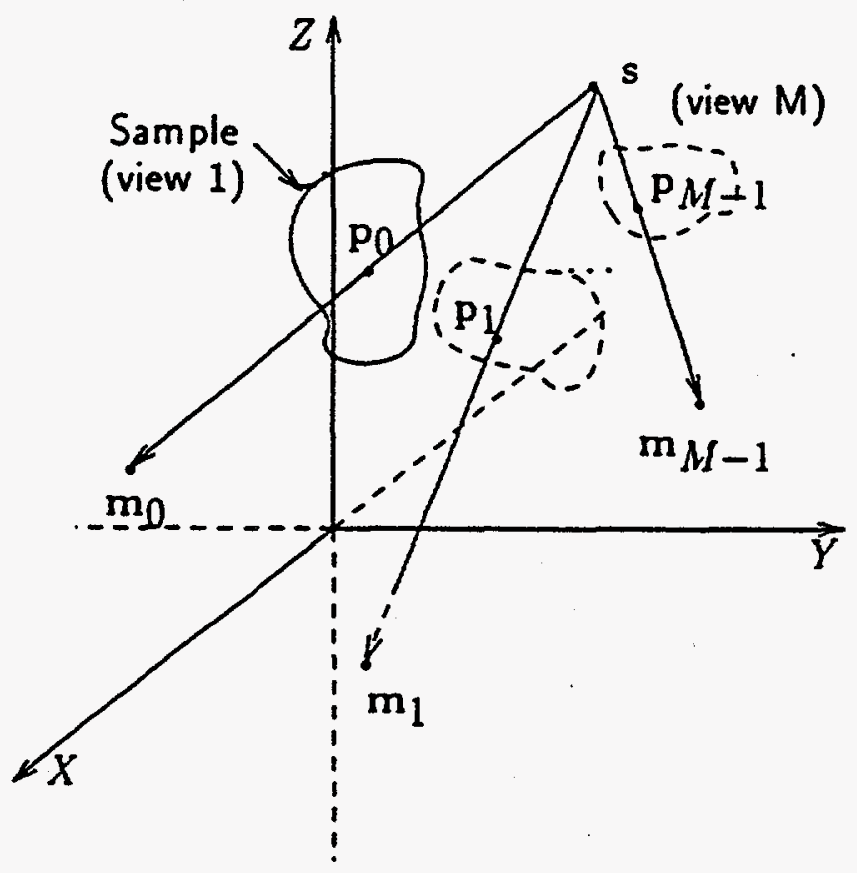

Figure A.2. Experiment geometry for generalized stereography.

derivation). The $\mathrm{X}$-ray source produces a cone beam which is modelled by a series of rays emanating from $s$. One of these rays passes through the point of interest $p_{0}$ (also called the range point) and casts a shadow (projection) on the $\mathrm{X}$-ray detector at the measurement point $\mathrm{m}_{0}$. Note that no particular detector geometry is assumed here, so the choice of $m_{0}$ in three dimensions is perfectly general. For example, the spherical surface of the image intensifier could be used directly rather than assuming a planar detector. The point $\mathrm{p}_{0}$ is embedded within a host material, so only $\mathrm{s}$ and $\mathrm{m}_{0}$ are known.

The goal of the reconstruction is to uniquely identify the 3-D coordinates of $\mathrm{p}_{0}$. It is known that $\mathrm{p}_{0}$ is collinear with the source point and measurement point since all three points lie on the same ray. Yet this is insufficient information to uniquely determine $p_{0}$ since any position along the ray satisfies the collinearity constraint. Additional information is obtained by translating $\mathrm{p}_{0}$ to a new location $p_{1}$, which selects a new ray and creates the measurement point $m_{1}$. $p_{0}$ is transformed to $p_{1}$ using the linear transformation $\mathrm{p}_{1}=A \mathrm{p}_{0}+\mathrm{b}_{1}$. Simple translation is accomplished by taking $\mathrm{A}_{1}$ as the identity matrix and $b_{1}$ as a non-zero vector. Rotation about a center of rotation $c$ is accomplished 
by subtracting $c$ (this takes $p_{0}$ to the origin), applying the rotation matrix $R$, and adding back $c$ to restore the original position:

$$
\begin{aligned}
p_{1} & =R\left(p_{0}-c\right)+c \\
& =\left(R \varphi_{0}-R c\right)+c \\
p_{1} & =R \varphi_{0}+(I-R) c
\end{aligned}
$$

For this transformation $A_{1}=R$ and $b_{1}=(I-R) c$.

The additional measurement point $m_{1}$ and knowledge of how $p_{1}$ is related to $p_{0}$ provides sufficient information to find $\mathrm{p}_{0}$. This information is summarized as follows:

Condition 1: $\mathrm{s}, \mathrm{p}_{0}$, and $\mathrm{m}_{0}$ are collinear,

Condition 2: $\mathrm{s}, \mathrm{p}_{1}$, and $\mathrm{m}_{1}$ are collinear, and

Condition 3: $p_{1}=A_{1} p_{0}+b_{1}$

Conditions 1 and 2 are constraints on the locations of the unknown point and its translate.

Condition 3 indicates how the constraints are coupled together.

Any three points $\mathrm{A}, \mathrm{B}$, and $\mathrm{C}$ are collinear when the cross product $\mathrm{AB} \times \mathrm{AC}$ equals zero. It will be convenient to define the cross product operation in matrix notation. In vector notation, the cross product of two vectors $\mathrm{A}$ and $\mathrm{B}$ is given by the determinant

where $\mathrm{i}, \mathrm{j}$, and $\mathrm{k}$ are unit vectors in the $\mathrm{X}, \mathrm{Y}$, and $\mathrm{Z}$ directions, respectively. Expanding this determinant yields

$$
A \times B=\left|\begin{array}{ccc}
i & j & k \\
A_{x} & A_{y} & A_{z} \\
B_{x} & B_{y} & B_{z}
\end{array}\right|
$$




$$
A \times B=\left(A_{y} B_{z}-A_{z} B_{y}\right) i-\left(A_{x} B_{z}-A_{z} B_{x}\right) j+\left(A_{x} B_{y}-A_{y} B_{x}\right) k
$$

Using the correspondence $a=\left[\begin{array}{lll}a_{1} & a_{2} & a_{3}\end{array}\right]^{\mathrm{T}} \leftrightarrow A=A_{x} i+A_{y} j+A_{z} k$, the above expansion is written in matrix notation as

$$
\left[\begin{array}{cc}
a_{2} b_{3} & -a_{3} b_{2} \\
-a_{1} b_{3}+a_{3} b_{1} \\
a_{1} b_{1} & -a_{2} b_{1}
\end{array}\right]=\left[\begin{array}{ccc}
0 & -a_{3} & a_{2} \\
a_{3} & 0 & -a_{1} \\
-a_{2} & a_{1} & 0
\end{array}\right]\left[\begin{array}{l}
b_{1} \\
b_{2} \\
b_{3}
\end{array}\right]
$$

The operator which takes the $3 \times 1$ vector a onto the $3 \times 3$ matrix premultiplying $b$ in the above equation is defined as $C_{x}$, where

$$
c_{x} \equiv\left[\begin{array}{ccc}
0 & -X_{3} & x_{2} \\
x_{3} & 0 & -x_{1} \\
-X_{2} & x_{1} & 0
\end{array}\right]
$$

Hence, $A \times B$ corresponds to $C_{a} b$.

Now, the collinearity constraint of Condition 1 is expressed by equating the cross product of the vector pointing from the measurement point to the source point $(\mathrm{s}-\mathrm{mo})$ and the vector pointing from the measurement point to the range point $\left(p_{\circ}-m_{o}\right)$ to zero:

$$
\begin{aligned}
C_{s-m_{0}}\left(p_{0}-m_{0}\right) & =0 \\
C_{s-m_{0}} p_{0} & =C_{s-m_{0}} m_{0}
\end{aligned}
$$


It is tempting to solve the above equation for po; however, the rank of $C_{x}$ [deñoted $\rho\left(C_{x}\right)$ ] is $\leq 2$, but $\mathrm{C}_{\mathrm{x}}$ is a $3 \times 3$ matrix. Thus, $\mathrm{C}_{\mathrm{x}}$ is not invertible, so $\mathrm{p}_{\mathrm{o}}$ cannot be determined uniquely. This further demonstrates the need for making more than one measurement to uniquely determine the range point.

Condition 2 is expressed in a like manner:

$$
c_{s-m_{1}} p_{1}=C_{s-m_{1}} m_{1}
$$

By Condition 3, $p_{1}=A_{1}$ po $+b_{1}$, so by substitution into Equation (A.8),

$$
\begin{aligned}
C_{s-m_{1}}\left(A_{1} p_{0}+b_{1}\right) & =C_{s-m_{1}} m_{1} \\
C_{s-m_{1}} A_{1} p_{0} & =C_{s-m_{1}}\left(m_{1}-b_{1}\right)
\end{aligned}
$$

Combining Equations (A.7) and (A.9) forms the system

$$
\left(\begin{array}{l}
C_{s-m_{0}} A_{0} \\
C_{s-m_{1}} A_{1}
\end{array}\right)\left(p_{0}\right)=\left(\begin{array}{l}
C_{s-m_{0}}\left(m_{0}-b_{0}\right) \\
C_{s-m_{1}}\left(m_{1}-b_{1}\right)
\end{array}\right)
$$

where $A_{0} \equiv I$ and $b_{0} \equiv 0$. This is an overdetermined system, so $p_{0}$ is a least-squares solution.

However, for the case of two measurements, the left-hand matrix has only six rows, so this particular system is only, slightly overdetermined. The "overdeterminedness" of the system is improved by including additional measurements $m_{i}$ obtained by applying a series of transformations to po to yield the set of range points $p_{i}=A_{i} p_{0}+b_{i}$. A total of $M$ measurements yields the general system 


$$
\left(\begin{array}{c}
C_{s-m_{0}} A_{0} \\
C_{s-m_{1}} A_{1} \\
C_{s-m_{2}} A_{2} \\
\vdots \\
C_{s-m_{M-1}} A_{M-1}
\end{array}\right)\left(P_{0}\right)=\left(\begin{array}{c}
c_{s-m_{0}}\left(m_{0}-b_{0}\right) \\
C_{s-m_{1}}\left(m_{1}-b_{1}\right) \\
C_{s-m_{2}}\left(m_{2}-b_{2}\right) \\
\vdots \\
C_{s-m_{M-1}}\left(m_{M-1}-b_{M-1}\right)
\end{array}\right)
$$

The left matrix premultiplying $\mathrm{p}_{0}$ is a $3 \mathrm{M} \times 3(\mathrm{M} \geq 2)$ rectangular matrix denoted $\mathrm{X}$. The right hand side is a $3 \mathrm{M} \times 1$ column vector denoted $\mathrm{y}$.

\section{System Inversion}

The system of Equation (A.11) is an overdetermined linear system of the form $X p=y$, where the zero subscript has been dropped to simplify the following discussion. Solving for $p$ requires computation of the matrix inverse of $\mathrm{X}$. $\mathrm{X}$ is rectangular, so only a generalized inverse $\mathrm{X}^{-}$ can be found. The solution vector is then $p=X y$. When $p\left(X^{T} X\right)=3$. $X^{T} X$ is a square matrix and is invertible. Thus, the most computationally straightforward solution is

$$
p=\left(X^{T} X\right)^{-1}\left(X^{T} y\right)
$$

Unfortunately, this method (known as solution of the normal equations) is prone to numerical instability, especially when X is ill-conditioned (Press et al., 1988). The singular value decomposition (SVD) (Press et al., 1988) approach is more numerically stable, and provides useful information concerning the quality of the solution vector $p$.

The SVD decomposes $\mathrm{X}$ as follows: 


$$
X=U W^{T}
$$

where $\mathrm{U}$ and $\mathrm{V}^{\mathrm{T}}$ are orthogonal matrices, and $\mathrm{W}$ is a diagonal matrix containing the singular values $w_{i}$. The solution vector $p$ is determined as

$$
\begin{aligned}
p & =X^{-} y \\
& =\left(U W^{T}\right)^{-1} y \\
& =\left(V^{T}\right)^{-1} w^{-1} U^{-1} y \\
p & =V W^{-1} U^{T} y
\end{aligned}
$$

The last step follows since the transpose of an orthogonal matrix is its inverse; $\mathrm{W}$ is diagonal, so $\mathrm{W}^{-1}$ is found by taking the reciprocal of the diagonal elements.

The condition number of the matrix $\mathrm{X}$ indicates to what extent $\mathrm{X}$ is ill-conditioned for the least-squares problem. The condition number is the ratio of the maximum and minimum singular values:

$$
C N=\frac{\omega_{\max }}{\omega_{\min }}
$$

$\mathrm{CN} \simeq 1$ indicates a well-conditioned $\mathrm{X}$ matrix, while a large value indicates it is ill-conditioned (Press et al., 1988). Furthermore, the sensitivity of the least-squares solution is proportional to the square of the condition number (Golub and Van Loan, 1989). In the laboratory software implementation, the condition number is included as an output to the user as a diagnostic to indicate how much confidence can be placed in the least-squares solution vector $p$. 


\section{Application of Model in RTR Environment}

This system computes the 3-D coordinates of a point $p_{0}$ given a measurement point $m_{0}$ derived from an initial view of the sample, a sequence of additional measurements $m_{1}, m_{2}, \ldots, m_{M-1}$ derived from a series of new sample orientations defined by $\left(A_{i}, b_{i}\right)$, and knowledge of the source location $s$. The sample positioner center of rotation is indicated by the $3 \times 1$ vector $c$. The angular position of the platter is denoted by $\theta$.

Figure A.3 shows a diagram of the coordinate systems used for the X-ray source, sample positioner, and detector. The global coordinate system $\left(\mathrm{X}_{8}, \mathrm{Y}_{\mathrm{g}}, \mathrm{Z}_{\mathrm{g}}\right)$ is centered on the face of the $\mathrm{X}$-ray detector. The source is located somewhere in the positive $\mathrm{Z}_{\mathrm{g}}$ half-space. A coordinate system local to the sample $\left(\mathrm{X}_{\mathrm{s}}, \mathrm{Y}_{\mathrm{s}}, \mathrm{Z}_{\mathrm{s}}\right)$ is placed with its origin at $\mathrm{c}$. Linear transformations are used to map device coordinates to the global coordinate system. For example, the sample positioner reports position in steps, and the display screen coordinates are reported in pixels. Both need to be converted into the global coordinate system in centimeter units.

The sample positioner lacks shaft encoders to provide an absolute position between invocations of the sample positioner program, so the positioner values are relative to the initial platter position $\mathrm{c}$ loaded from the laboratory geometry file. Once the sample is attached to the platter, the position is adjusted to obtain an initial view. A projection of a point of interest is defined from the image using a mouse-guided cursor to give the first measurement $m_{0}=\left[m_{1} m_{2} 0\right]^{\mathrm{T}}\left(m_{3}\right.$ is always zero since the detector plane lies in the $\mathrm{X}_{\mathrm{g}} \mathrm{Y}_{\mathrm{g}}$ plane). The current position of the platter is recorded as $\mathrm{c}_{0}$ $=c+\Delta c_{0}$ and $\theta_{0}$. The sample is translated and/or rotated to a new view.

Once $m_{1}$ is defined from the image, the sample position is recorded as $c_{1}=c+\Delta c_{1}$ and $\theta_{1}$. The linear transformation linking the range points is $p_{1}=R\left(p_{0}-c_{0}\right)+c_{1}$. Conceptually, this transformation takes the range point po back to the initial center of rotation $c_{0}$, applies the rotation matrix $R$ (a function of the change in rotation $\theta_{1}-\theta_{0}$ ), and translates it back to the new center of rotation $c_{1}$. In terms of the general transformation defined by $\left(A_{1}, b_{1}\right)$ : 


$$
A_{1}=R\left(\theta_{1}-\theta_{0}\right)=\left[\begin{array}{ccc}
\cos \left(\theta_{1}-\theta_{0}\right) & 0 & \sin \left(\theta_{1} \theta_{0}\right) \\
0 & 1 & 0 \\
-\sin \left(\theta_{1}-\theta_{0}\right) & 0 & \cos \left(\theta_{1}-\theta_{0}\right)
\end{array}\right]
$$

$$
b_{1}=\left(c+\Delta c_{1}\right)-A_{1}\left(c+\Delta c_{\curvearrowright}\right)
$$

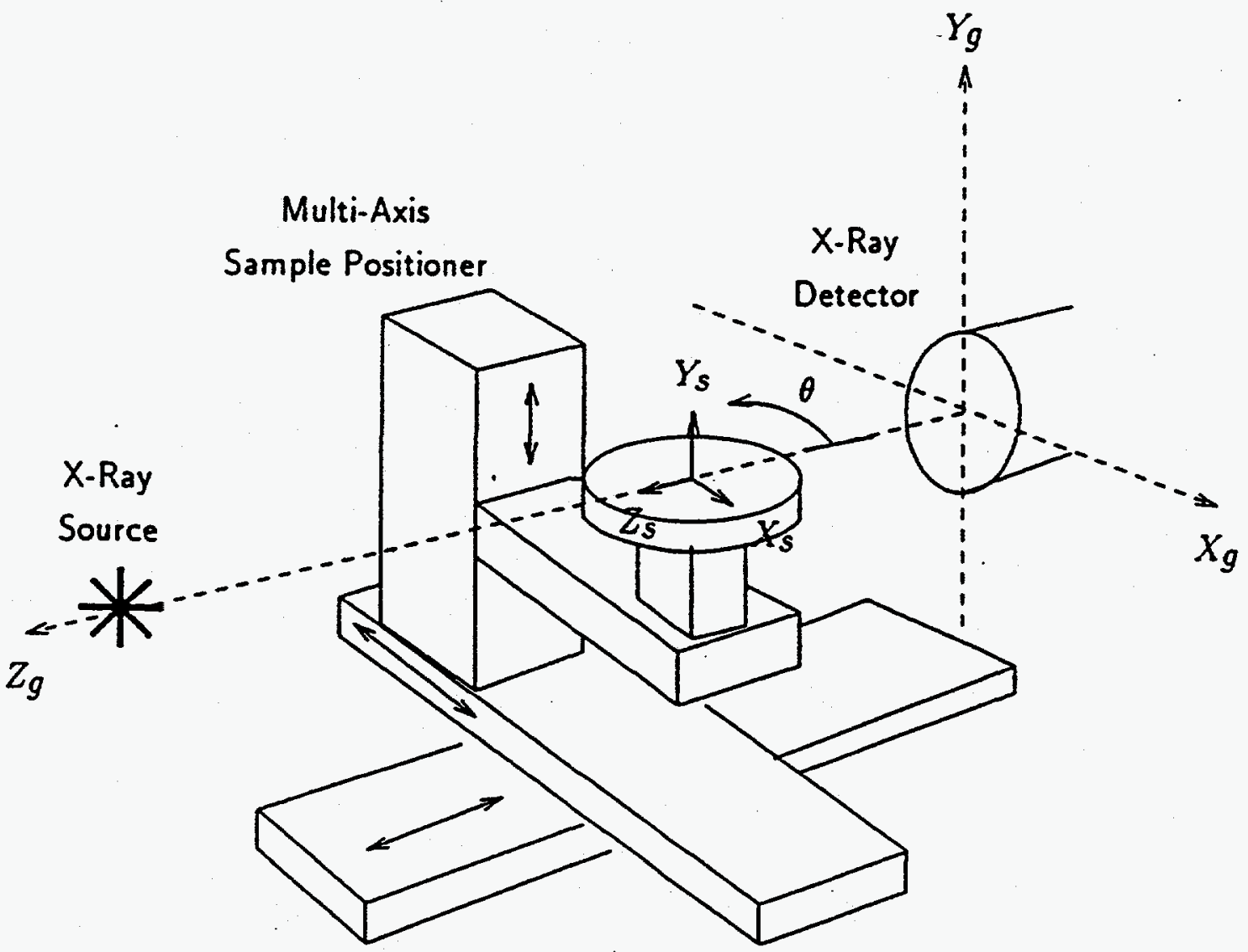

Figure A.3. Global and sample coordinate systems for RTR laboratory. 
The matrix $R(\theta)$ applies a rotation about the $Y_{s}$ axis. This process is repeated until $M \geq 2$ measurements are made. The quantities $s, m_{i}, A_{i}$, and $b_{i}$ are organized into the system of Equation (A.11), which is subsequently inverted to solve for the range point $p_{0}$. The entire process is applied to an arbitrary number of range points within the sample.

Flaw measurements are made by selecting endpoints of crack features. Complex cracks are measured by selecting vertices within the crack image as range points. Once the 3-D coordinates are determined, the crack is reconstructed by connecting piecewise linear segments between the range points. The orientation of the crack is completely determined by the points $\mathrm{p}_{0 \mathrm{i}}$. The length of the crack is computed by summing the lengths as

$$
L=\sum_{i=1}^{M-1}\left|p_{0 i}-p_{o i-1}\right|
$$

This feature was implemented into the 3-D reconstruction program to allow the user to measure the length between an arbitrary sequence of points.

The centroid of void-like flaws and inclusions can be identified as well. The 3-D centroid projects closely to the 2-D centroid of the projection on the detector. For a sphere of uniform density, the distance between the centroid of the 2-D elliptical projection and the projection of the 3-D sphere centroid is (McNeil, 1966)

$$
\Delta x=(d / 2)[\tan (\theta-\phi)+\tan (\theta+\phi)-2 \tan \theta]
$$

where $d$ is the source-to-detector distance, $\theta$ is the angle between a source ray passing through the sphere centroid and a source ray normal to the detector, and $2 \phi$ is the angle subtended by the sphere boundary (see Figure A.4). The distance $\Delta \mathrm{x}$ is zero when the sphere is on-axis with the source $(\theta=0) . \Delta x$ increases slightly off-axis. McNeil used a $1 / 4 \mathrm{in} .(0.64 \mathrm{~cm})$ sphere with a source/detector distance $\mathrm{d}=36^{\prime \prime}(91 \mathrm{~cm})$ and $6=14 \mathrm{in.} / 02 \mathrm{ft}\left(\phi=0^{\circ} 12^{\prime}\right.$ follows from the $1 / 4^{\prime \prime}$ sphere) to get $\Delta \mathrm{x}=0.00012 \mathrm{in}$. $(0.00030 \mathrm{~cm})$. Thus, the error is negligible, considering that the 


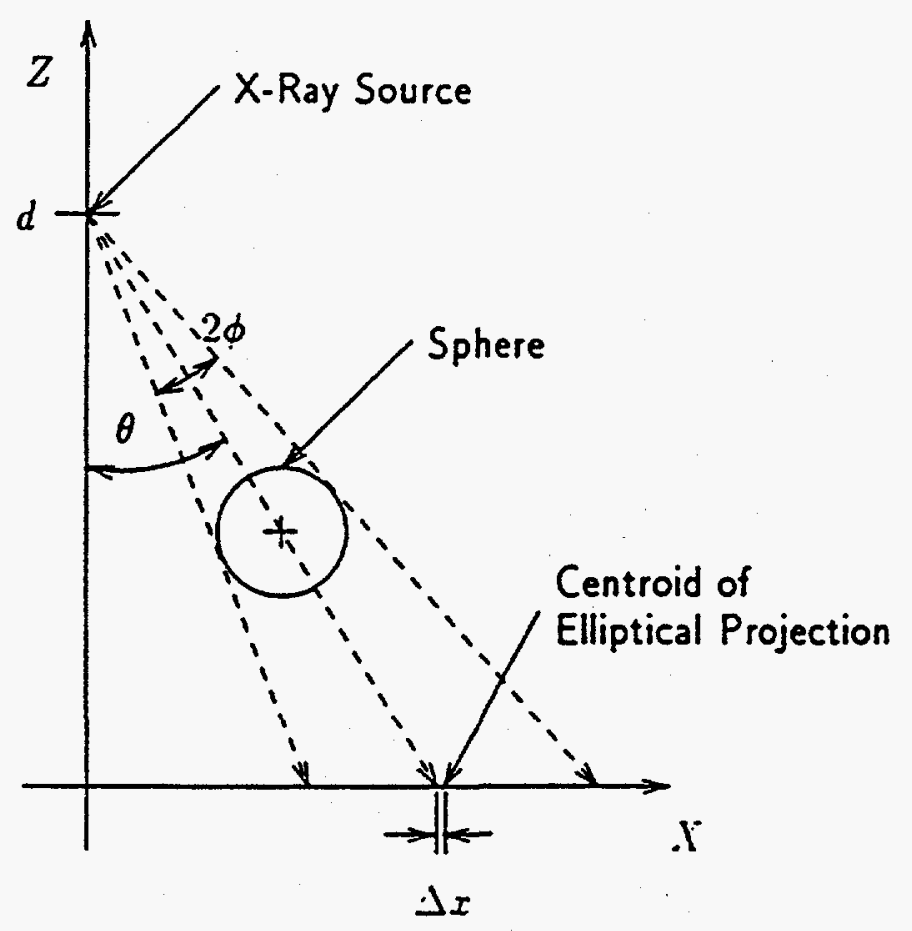

Figure A.4. Error in determining 3-D centroid from 2-D projection.

effective width of a pixel in the X-ray detector is $0.017 \mathrm{~cm}$ (at 60 pixels $/ \mathrm{cm}$ sampling frequency). Limits on the void's boundaries are determined by making 2-D measurements from a series of sample rotations. More accurate modeling of the flaw shape is possible (Wallingford, 1990), although this requires the gray values of the projection to be used. The image intensifier has a wide variety of radiometric aberrations which would need to be taken into account to make this work successfully.

\section{Simulation of 3-D Measurement System}

A simulator was developed to make detailed analyses of the 3-D measurement system. The simulator is split into a forward model and an inverse model. The inverse model is the same as that implemented in the real-time laboratory. It uses $\mathrm{s}, \mathrm{c}, \mathrm{m}_{\mathrm{i}}$, and a list of sample manipulations to calculate $\mathrm{p}_{0}$. The forward model uses the same information, except $\mathrm{p}_{0}$ is input and the measurements $\mathrm{m}_{\mathrm{i}}$ are output. These models are used as the basis of a Monte-Carlo simulation to study the effects of noise and bias on the inverse solution. Simulation was used since interaction of variables and lack of an analytic expression for the inversion process would otherwise make this analysis difficult. 


\section{Forward model equations}

The same collinearity constraints are used for the forward model, except now the range point $\mathrm{p}_{0}$ is given and the measurement $\mathrm{m}_{0}$ needs to be determined. The collinearity of these points with the source is expressed as

$$
\begin{aligned}
C_{s-p 0}\left(m_{0}-p_{0}\right) & =0 \\
C_{s-p 0} m_{0} & =C_{s}-p_{0} p_{0} \\
C_{s-p 0} m_{0} & =c_{s p_{0}}
\end{aligned}
$$

The transformations $\left(A_{i}, b_{i}\right)$ are computed using the same formulas as for the 3-D inversion [Equations (A.16) and (A.17)]. These are applied to $\mathrm{p}_{0}$ to find the translates

The $\mathrm{Z}$ component of $\mathrm{m}_{\mathrm{i}}$ is constrained to the detector surface. A planar surface in the $\mathrm{XY}$ plane is selected, so $\mathrm{m}_{\mathrm{i3}}=\mathbf{0}$ for all measurements (a planar surface is justified since the X-ray detector is calibrated to emulate a linear planar detector). This reduces the number of unknowns to two, so Equation (A.20) can be solved uniquely. Expanding both sides of Equation (A.20) results in

$$
\left[\begin{array}{c}
-\left(s_{3}-p_{03}\right) m_{02} \\
\left(s_{3}-p_{03}\right) m_{01} \\
-\left(s_{2}-p_{02}\right) m_{01}+\left(s_{1}-p_{01}\right) m_{02}
\end{array}\right]=\left[\begin{array}{c}
-s_{3} p_{02}+s_{2} p_{03} \\
s_{3} p_{01}-s_{1} p_{03} \\
-s_{2} p_{01}+s_{1} p_{02}
\end{array}\right]
$$

Adding the first two rows results in the following system:

$$
\left[\begin{array}{c}
\left(s_{3}-p_{03}\right)-\left(s_{3}-p_{03}\right) \\
-\left(s_{2}-p_{02}\right)\left(s_{1}-p_{01}\right.
\end{array}\right]\left[\begin{array}{l}
m_{01} \\
m_{02}
\end{array}\right]=\left[\begin{array}{c}
s_{3}\left(p_{01}-p_{02}\right)+\left(s_{2}-s_{1}\right) p_{03} \\
s_{1} p_{02}-s_{2} p_{01}
\end{array}\right]
$$


This is a full rank system which can be solved for the measurement $\mathrm{X}$ and $\mathrm{Y}^{-}$coordinates. This technique is applied to each $p_{i}$ to calculate the remaining measurement points.

\section{Simulation results}

The simulator was implemented using the modules shown in Figure A.5. The ellipses indicate user specified parameters to define the laboratory setup (X-ray source position and sample positioner location), the range point location, and the variances and biases to be used to perturb the positions of the $\mathrm{X}$-ray source, sample center of rotation, and screen measurements. The rectangles indicate the major sections of the simulator itself. The screen measurements $m_{i}$ are computed using the forward model described in the previous section. These measurements are considered to be "exact" since no noise or bias has been added yet. In the next step, more "realistic" measurements of the system are created. A normal random number generator is used to creafe measurement noise; for each measurement quantity, the random number is scaled by a standard deviation and offset by a bias appropriate to that quantity. The X-ray source and sample position-measurement noise is assumed to be continuous, while the screen measurements are quantized to emulate the behavior of the digitizer.

The perturbed values are applied to the same algorithm that is used to make the 3-D measurements in the laboratory. This routine generates an estimate of the range point, which is saved to an array. The simulator goes back and calculates a new set of noise values, which in turn are applied to the range point calculator to generate another estimate of the range point. This process is repeated many times (usually 100) to build up a distribution of estimates. The average and standard deviation of this distribution are computed, and bias is determined by comparing the average value of the estimate to the "true" value specified earlier by the user.

The error in estimating the $Z$ component (depth) of the range point is of most interest, since the $\mathrm{X}$ and $\mathrm{Y}$ components are easily calculated from only a single 2-D projection image. Measurement errors stem from four sources: 


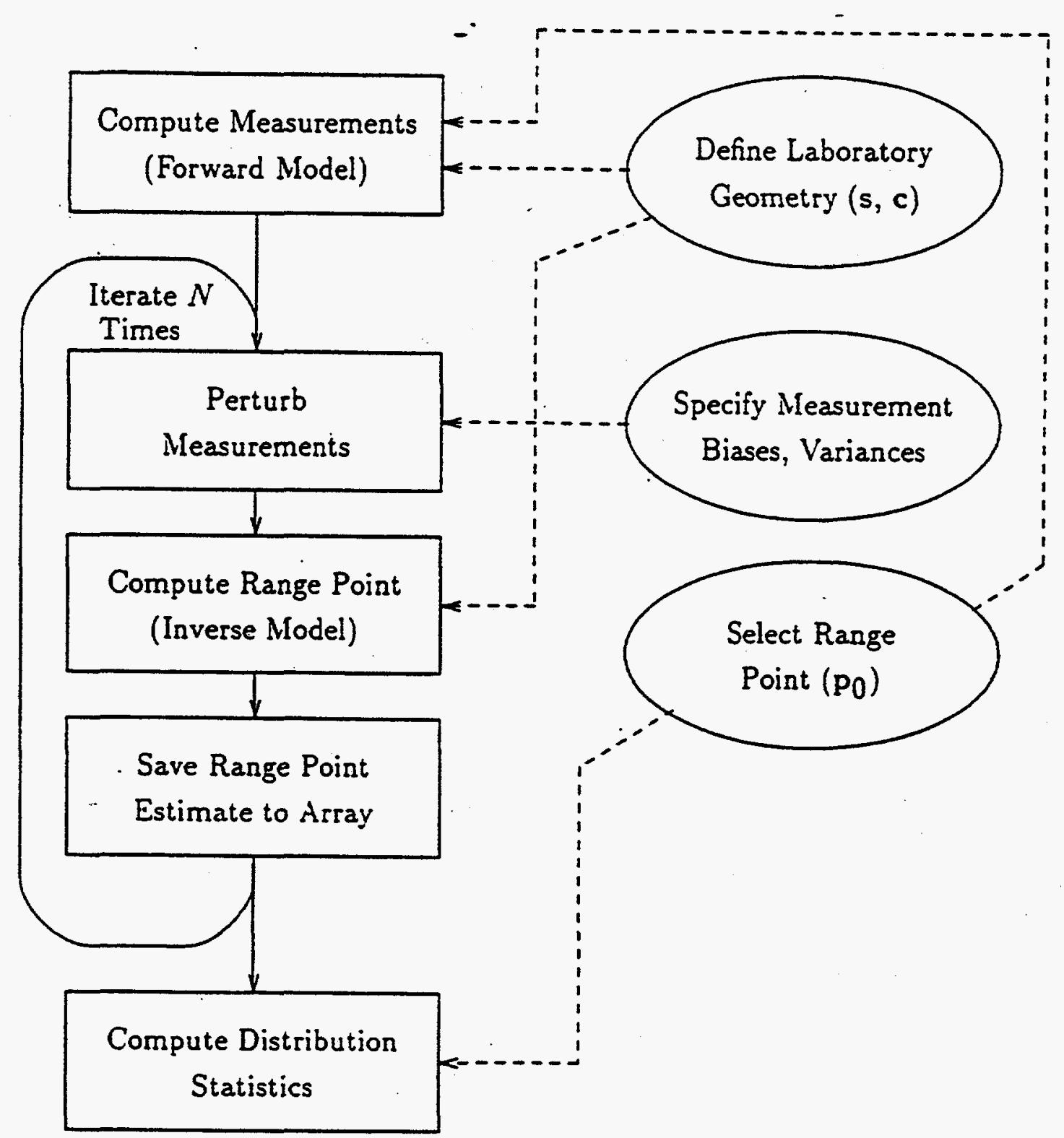

Figure A.5. Flow diagram of 3-D measurement simulator.

1. Determining the 3-D position of the X-ray source,

2. Determining the 3-D center of rotation of the sample positioner platter,

3. Movement of the sample from one view to the next, and

4. Determining the measurement points from the display screen. 
Error Source (3) is negligible in this system due to the high precision and accuracy of the sample positioner. The laboratory source/positioner/detector geometry remains fixed once the measurement is in progress, so Error Sources (1) and (2) contribute a systematic error or bias to the measurement. Selection of measurement points from the display screen is the only variation encountered during repetition of the same experiment, so Error Source (4) contributes the random error to the depth measurement. Consequently, the simulation studies were used to determine the influence of the following factors on the systematic and random error components of the depth measurement:

- Sensitivity to random measurement errors as a function of:

1. change in position between two views (translation)

2. change in angle between two views (rotation)

3. distance from center of rotation

4. number of views between a fixed angle of rotation

5. sample/detector distance (magnification)

- Sensitivity to bias errors in:

1. source $Z$ position

2. sample positioner center of rotation $\mathrm{Z}$ position

Some results from Appendix B are used to make the simulations applicable to the laboratory measurements performed there. A precise method was developed to calculate the $\mathrm{X}$ and $\mathrm{Y}$ components of $s$ and $c$ once the $Z$ component was known, so the $Z$ component measurement dominates the error in determining these quantities. The $Z$ position was determined using a tape measure, which was accurate to within $\pm 1 / 16^{\prime \prime}( \pm 0.16 \mathrm{~cm})$. The standard deviation associated with measuring feature points from the display screen was found to be $0.5-0.8$ pixels. The simulations use a standard deviation of 0.7 pixels for both $X$ and $Y$ measurements; this corresponds to $0.012 \mathrm{~cm}$ resulting from the $60 \mathrm{pixel} / \mathrm{cm}$ sampling frequency used to digitize the real-time image. A constant standard deviation for the measurement noise is justified since in practice image processing routines are used to enhance the features of interest, so the operator experiences the same level of uncertainty in selecting these features from one view to the next. 
The nominal setup for the simulations places the $X$-ray source $100 \mathrm{~cm}^{-}$from the detector. Magnifications of 4 times were most commonly used for the samples in Appendix B; $1 \mathrm{x}$ and $20 \mathrm{x}$ magnifications are also investigated here.

Translation. A two-view translation experiment was set up using the geometry indicated in Table A.1. The table lists the quantity of interest, its nominal value, and the standard deviation and bias of the random noise added to the nominal value to make the perturbed measurement value.

Here, the $X$-ray source was placed on the $Z$ axis $100 \mathrm{~cm}$ from the detector, the positioner center was placed on the $\mathrm{Z}$ axis $60 \mathrm{~cm}$ from the detector (which results in $4 x$ magnification), and the range point was located on the $Z$ axis $2 \mathrm{~cm}$ from the center of rotation. Measurement noise was added only to the display screen measurements. The initial view was always made with the range point positioned as shown in the table, and the second view was made after translating the range point in the $+X$ direction by an amount varying from 0 to $20 \mathrm{~cm}$. Both the standard deviation of the range point depth measurement and the condition number of the inversion are plotted as functions of the change in position (Figure A.6). Note that the sample had to be translated at least $15 \mathrm{~cm}$ to achieve a depth standard deviation below $0.02 \mathrm{~cm}$. This much translation is impractical for a single-detector setup since the detector has limited field of view. In fact, the laboratory setup of Chapter 5 only permitted a translation of $1.5 \mathrm{~cm}$ at $4 \mathrm{x}$ magnification, so the simulation predicts at least an order of magnitude higher error for a practical geometry. A second detector would be required to image the translated sample, which obviously complicates the laboratory setup.

Table A.1. Simulation parameters for translation experiment, $\sim \mathrm{x}$ magnification.

\begin{tabular}{||c|c|c|c||}
\hline Quantity & $\begin{array}{c}\text { Nominal Value } \\
(\mathrm{cm})\end{array}$ & $\begin{array}{c}\text { Standard Deviation } \\
(\mathrm{cm})\end{array}$ & $\begin{array}{c}\text { Bias } \\
(\mathrm{cm})\end{array}$ \\
\hline $\mathrm{s}$ & {$\left[\begin{array}{lll}0 & 0 & 100\end{array}\right]^{\mathrm{T}}$} & {$\left[\begin{array}{lll}0 & 0 & 0\end{array}\right]^{\mathrm{T}}$} & {$\left[\begin{array}{lll}0 & 0 & 0\end{array}\right]^{\mathrm{T}}$} \\
\hline $\mathrm{c}$ & {$\left[\begin{array}{lll}0 & 0 & 6\end{array}\right]^{\mathrm{T}}$} & {$\left[\begin{array}{llll}0 & 0 & 0\end{array}\right]^{\mathrm{T}}$} & {$\left[\begin{array}{lll}0 & 0 & 0\end{array}\right]^{\mathrm{T}}$} \\
\hline $\mathrm{p}_{\mathrm{s}}$ & {$\left[\begin{array}{llll}0 & 0 & 58\end{array}\right]^{\mathrm{T}}$} & - & - \\
\hline $\mathrm{m}$ & - & {$\left[\begin{array}{lllll}0.012 & 0.012 & 0\end{array}\right]^{\mathrm{T}}$} & {$\left[\begin{array}{lll}0 & 0 & 0\end{array}\right]^{\mathrm{T}}$} \\
\hline
\end{tabular}




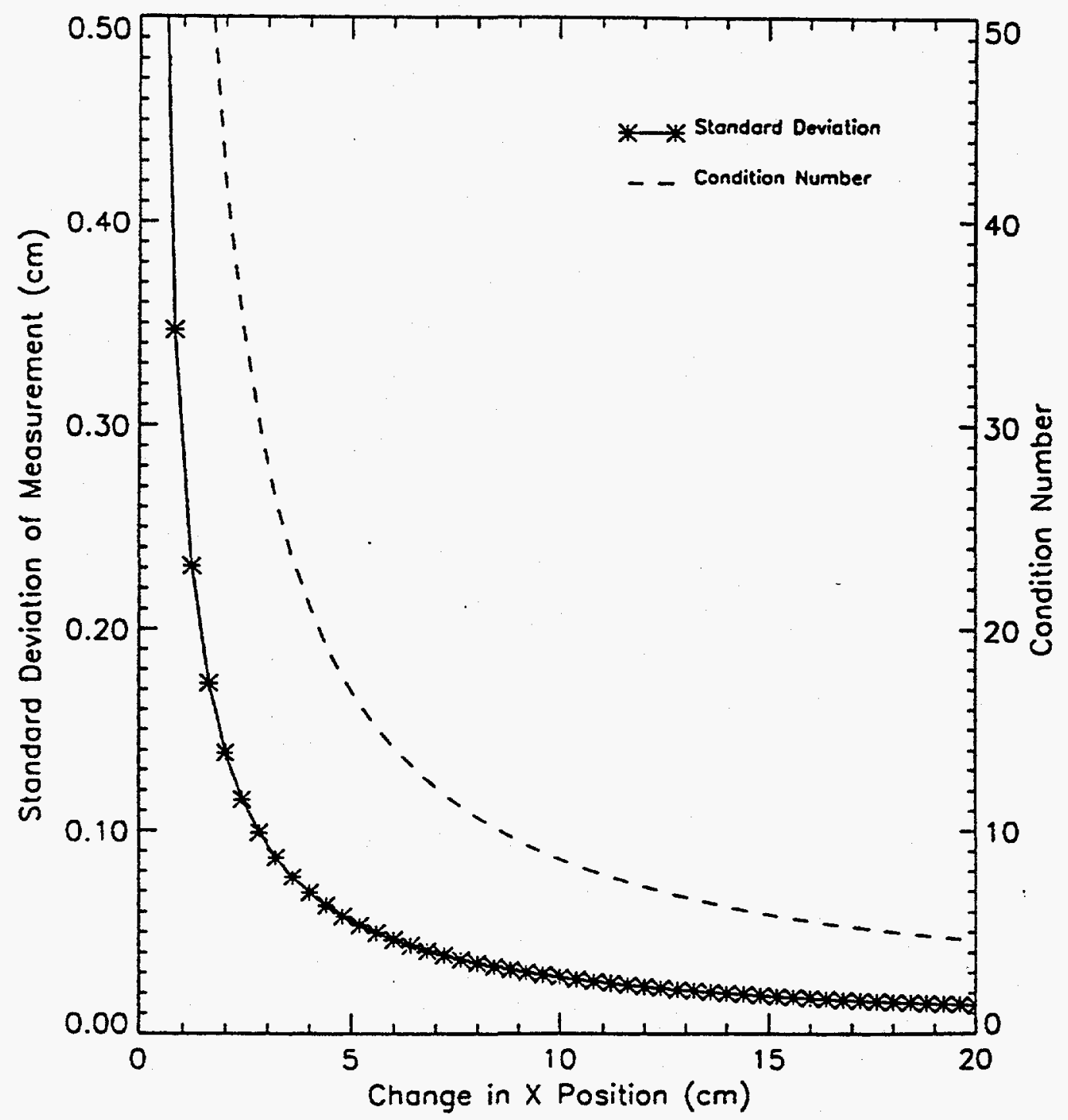

Figure A.6. Error sensitivity as a function of translation amount.

Translation-only measurements result in an ill-conditioned $\mathrm{X}$ matrix for two reasons. First, the long source-to-detector distance dominates the difference $s-m_{j}$ since $\|s\|$ is usually $100 \mathrm{~cm}$, and $\left\|\mathrm{m}_{\mathrm{i}}\right\|$ is about $4 \mathrm{~cm}$ due to the limited detector field of view. Second, the range point transformation matrix $A_{i}$ is identity, so the additional rows added to $X$ are nearly the same as the first three rows found by the initial measurement. For example, a $2 \mathrm{~cm}$ sample translation with the geometry of 


$$
X=\left[\begin{array}{ccc}
0 & -100 & 0 \\
-100 & 0 & 0 \\
0 & 0 & 0 \\
0 & -100 & 0 \\
100 & 0 & 4.76 \\
0 & -4.76 & 0
\end{array}\right]
$$

The first three rows result from the initial view, and the last three rows are obtained after translating to the second view. Rows 3, 4, and 6 are all linearly dependent on Row 1, and Row 5 is only marginally independent from Row 2 . Consequently, the $X_{53}$ value is the only value keeping $\rho(X)=$ 3 ; $X_{53}$ has to be made fairly large to make $X$ better conditioned. As will be seen, using a rotation matrix for $A_{i}$ changes all the values in the last three rows of $X$ to make them linearly independent from the first three rows, making $X$ well-conditioned for inversion while keeping the simple geometry of a single fixed detector.

Rotation. The ability to rotate the sample is one of the primary advantages of this system over a translation-only system. A two-view rotation experiment was set up using the same geometry as the translation experiment (Table A.1). As before, the geometry shown in the table was used for the initial view. The second view was made by rotating the range point in the $+\theta$ direction in the range $0-360^{\circ}$. The depth standard deviation and condition number are plotted as a function of change in angle in Figure A.7.

Here, a $20^{\circ}$ rotation was sufficient to get an error below $0.02 \mathrm{~cm}$ (this required a $15 \mathrm{~cm}$ translation for the previous experiment), and errors less than $0.01 \mathrm{~cm}$ were typical over the majority of rotation angles. The measurement was ill-conditioned using small angular changes, but has also ill-conditioned at $180^{\circ}$ rotation. Using small angular changes does not substantially change the last three rows of $\mathrm{X}$, so the ill-conditioned nature of this situation is evident. However, a rotation of $180^{\circ}$ results in the following matrix: 


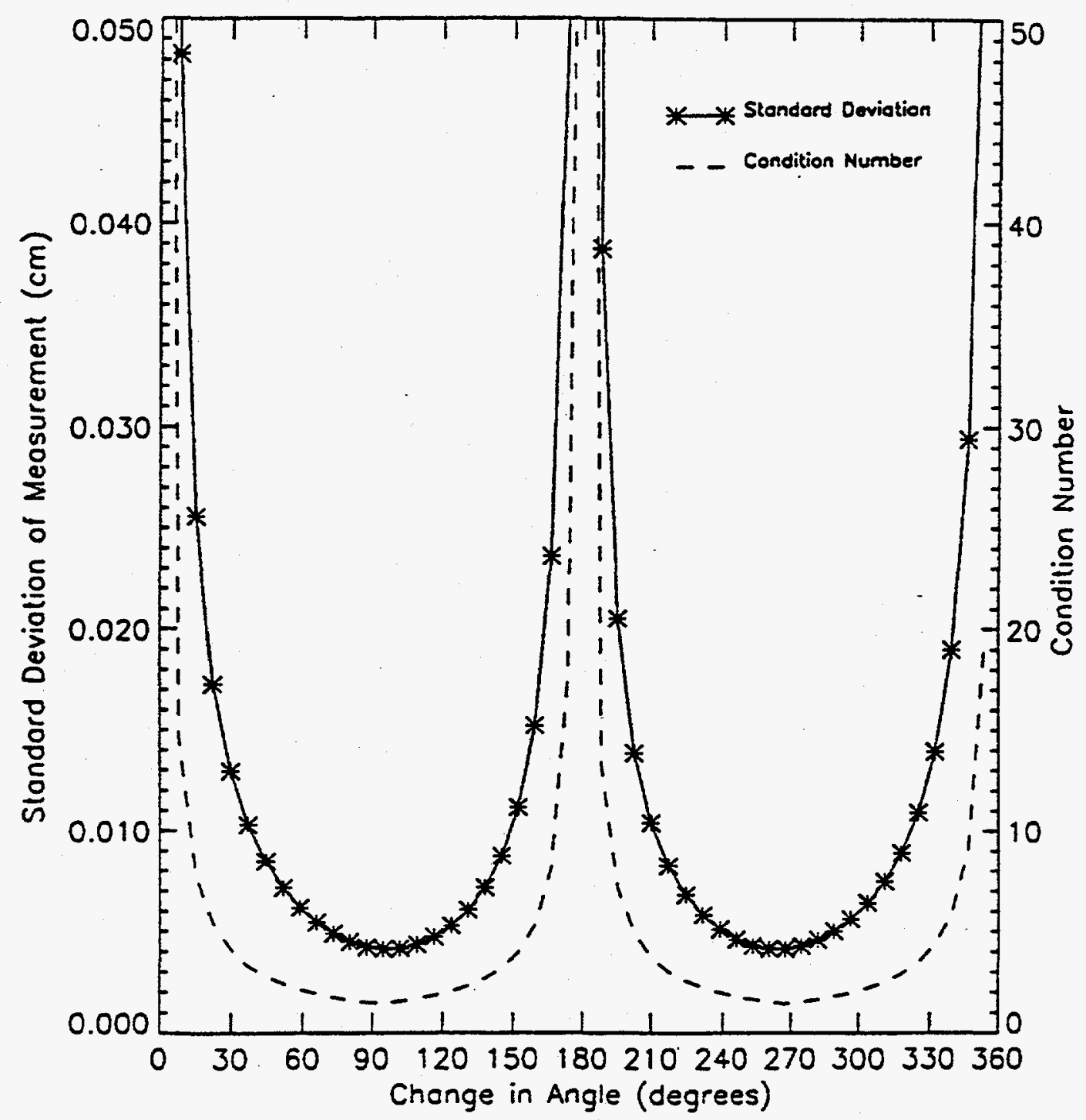

Figure A.7. Error sensitivity as a function of rotation amount.

$$
X=\left[\begin{array}{ccc}
0 & -100 & 0 \\
100 & 0 & 0 \\
0 & 0 & 0 \\
0 & -100 & 0 \\
-100 & 0 & 0 \\
0 & 0 & 0
\end{array}\right]
$$


Here the rank of $X$ is 2 , so the inversion cannot converge to a unique solution. This problem is due to the symmetry of placing $s, c$, and po all on the $Z$ axis. By moving the $X$-ray source off the $Z$ axis to $s=\left[\begin{array}{lll}20 & 30 & 100\end{array}\right]^{\mathrm{T}}$ the $\mathrm{X}$ matrix becomes:

$$
X=\left[\begin{array}{ccc}
0 & -100 & 71.4 \\
100 & 0 & -47.6 \\
-71.4 & 47.6 & 0 \\
0 & -100 & -78.9 \\
-100 & 0 & 52.6 \\
78.9 & 52.6 & 0
\end{array}\right]
$$

This matrix has $\mathrm{CN}=1.8$, indicating it is well-conditioned. The effect of using the off-axis $\mathrm{X}$-ray source as a function of change in rotation angle is plotted in Figure A.8.

In comparison to translation, rotation was found to be superior to achieve a given level of depth error. As a result, rotation-only measurements are emphasized in this work since they are more efficient.

Distance from center of rotation. The same geometry as the rotation experiment (Table A.1) was used here. The initial $Z$ position of $p_{0}$ was varied from $58 \mathrm{~cm}$ to $62 \mathrm{~cm}$ (i.e., $\pm 2 \mathrm{~cm}$ on either side of $\mathrm{c}$ ). The depth standard deviation in determining the range point position was $0.013 \mathrm{~cm}$ at $\mathrm{p}_{03}$ $=58 \mathrm{~cm}$ and $0.011 \mathrm{~cm}$ at $p_{03}=62 \mathrm{~cm}$. The error decreased monotonically between these two extremes, rather than reaching a minimum at zero distance from the center. Overall, the change was minimal; the trend of decreasing error as the point was moved closer to the source suggests the change was due to increased magnification. Using a $1 x$ configuration $\left(s=\left[\begin{array}{lll}0 & 0 & 100\end{array}\right)^{\mathrm{T}}, \mathrm{c}=\left[\begin{array}{lll}0 & 0 & 5\end{array}\right]^{\mathrm{T}}\right)$ with $p_{03}$ varying from $3 \mathrm{~cm}$ to $7 \mathrm{~cm}$ resulted in depth standard deviations of $0.029 \mathrm{~cm}$ and $0.027 \mathrm{~cm}$, respectively. Again, the change was minimal, and the error was less at the higher magnification condition of $\mathrm{p}_{03}=7 \mathrm{~cm}$. Thus, the variance in depth estimation was not found to be a function of distance from the center of rotation. 


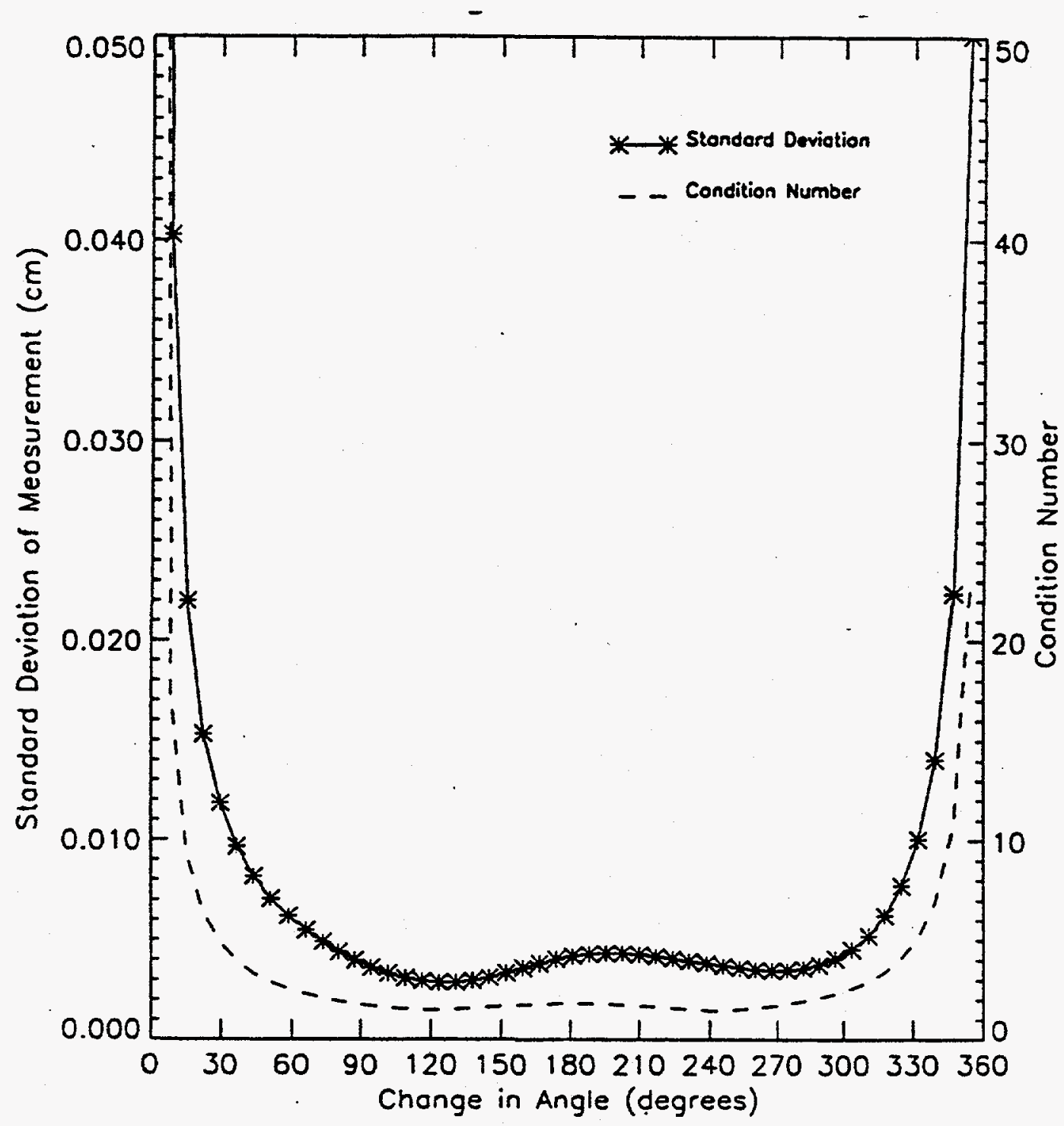

Figure A.8. Measured depth standard deviation due to change in angle (X-ray source off the $\mathrm{Z}$ axis).

Number of views between fixed angle of rotation. So far, only two-view experiments have been considered. The system in Equation (A.11) can support $M$ measurements, so this case was examined for a rotation experiment. The total rotation angle $\Delta \theta_{\mathrm{T}}$ was kept fixed, and multiple views were made by dividing this angle into equal sub-angles. The objective was to determine whether using the intermediate views could improve upon a two-view rotation experiment. Both a well-conditioned two-view experiment $\left(\Delta \theta_{\mathrm{T}}=30^{\circ}\right)$ and an ill-conditioned experiment $\left(\Delta \theta_{\mathrm{T}}=5^{\circ}\right)$ were considered. The $4 \mathrm{x}$ geometry of Table A.1 was used for both tests. Table A.2 lists the depth standard deviations and condition numbers as the number of views was increased from two to six. 


\begin{tabular}{|c|c|c|}
\hline \hline Table A.2. Simulation results for multi-vie-v rotation, with $30^{\circ}$ total rotation. \\
\hline Number of Views & $\begin{array}{c}\text { Depth Standard Deviation } \\
(\mathrm{cm})\end{array}$ & $\mathrm{CN}$ \\
\hline 2 & 0.013 & 4.04 \\
\hline 3 & 0.015 & 4.95 \\
\hline 4 & 0.012 & 5.42 \\
\hline 5 & 0.012 & 5.71 \\
\hline 6 & 0.011 & 5.90 \\
\hline
\end{tabular}

The decrease in error from two views to six views was $0.003 \mathrm{~cm}$, which is not a substantial improvement considering the amount of extra work required to obtain the additional data. Furthermore, the decrease was not monotonic since the error went up slightly from two views to three views.

The results for the ill-conditioned experiment are indicated in Table A.3. Here, the overall decrease was only $0.012 \mathrm{~cm}$, which represents a $15 \%$ reduction in error over the initial error of $0.079 \mathrm{~cm}$.

In both cases, it is evident that accumulating intermediate views can somewhat reduce the errors associated with measuring depth, but this marginal improvement needs to be weighed against the cost of collecting the extra data. In situations where angular rotations are limited to small angles, collecting intermediate views does not substantially improve the estimate. Thus, two-view experiments with a rotation of at least $20^{\circ}$ are sufficient to achieve good results.

Magnification: The effect of magnification on a two-view rotation experiment was considered for the cases of $1 \mathrm{x}$ and $20 \mathrm{x}$. Magnification effectively increases resolution and reduces display screen measurement noise since the larger object is easier to see. This improvement is paid for by reduced field of view, so less material can be inspected. Tables A.4 and A.5 list the laboratory geometries for the $1 x$ and $20 x$ configurations, respectively. 


\begin{tabular}{|c|c|c||}
\hline \hline Table A.3. Simulation results for multi-view rotation, with $5^{\circ}$ total rotation. \\
\hline Number of Views & $\begin{array}{c}\text { Depth Standard Deviation } \\
(\mathrm{cm})\end{array}$ & $\mathrm{CN}$ \\
\hline 2 & 0.079 & 24.07 \\
\hline 3 & 0.091 & 29.48 \\
\hline 4 & 0.071 & 32.23 \\
\hline 5 & 0.074 & 34.07 \\
\hline 6 & 0.067 & 35.23 \\
\hline
\end{tabular}

Table A.4. Simulation parameters for rotation experiment, $1 \mathrm{x}$ magnification.

\begin{tabular}{|c|c|c|c|}
\hline Quantity & $\begin{array}{c}\text { Nominal Value } \\
(\mathrm{cm})\end{array}$ & $\begin{array}{c}\text { Standard Deviation } \\
(\mathrm{cm})\end{array}$ & $\begin{array}{c}\text { Bias } \\
(\mathrm{cm})\end{array}$ \\
\hline $\mathrm{s}$ & {$\left[\begin{array}{lll}0 & 0 & 100\end{array}\right]^{\mathrm{T}}$} & {$\left[\begin{array}{lll}0 & 0 & 0\end{array}\right]^{\mathrm{T}}$} & {$\left[\begin{array}{lll}0 & 0 & 0\end{array}\right]^{\mathrm{T}}$} \\
\hline $\mathrm{c}$ & {$\left[\begin{array}{lll}0 & 0 & 3\end{array}\right]^{\mathrm{T}}$} & {$\left[\begin{array}{lll}0 & 0 & 0\end{array}\right]^{\mathrm{T}}$} & {$\left[\begin{array}{lll}0 & 0 & 0\end{array}\right]^{\mathrm{T}}$} \\
\hline $\mathrm{p}_{\mathrm{o}}$ & {$\left[\begin{array}{lll}0 & 0 & 1\end{array}\right]^{\mathrm{T}}$} & - & - \\
\hline $\mathrm{m}_{\mathrm{i}}$ & -- & {$\left[\begin{array}{llll}0.012 & 0.012 & 0\end{array}\right]^{\mathrm{T}}$} & {$\left[\begin{array}{lll}0 & 0 & 0\end{array}\right]^{\mathrm{T}}$} \\
\hline
\end{tabular}

Table A.5. Simulation parameters for rotation experiment, 20x magnification.

\begin{tabular}{|c|c|c|c|}
\hline Quantity & $\begin{array}{c}\text { Nominal Value } \\
(\mathrm{cm})\end{array}$ & $\begin{array}{c}\text { Standard Deviation } \\
(\mathrm{cm})\end{array}$ & $\begin{array}{c}\text { Bias } \\
(\mathrm{cm})\end{array}$ \\
\hline $\mathrm{s}$ & {$\left[\begin{array}{lll}0 & 0 & 100\end{array}\right]^{\mathrm{T}}$} & {$\left[\begin{array}{lll}0 & 0 & 0\end{array}\right]^{\mathrm{T}}$} & {$\left[\begin{array}{lll}0 & 0 & 0\end{array}\right]^{\mathrm{T}}$} \\
\hline $\mathrm{c}$ & {$\left[\begin{array}{lll}0 & 0 & 95\end{array}\right]^{\mathrm{T}}$} & {$\left[\begin{array}{lll}0 & 0 & 0\end{array}\right]^{\mathrm{T}}$} & {$\left[\begin{array}{lll}0 & 0 & 0\end{array}\right]^{\mathrm{T}}$} \\
\hline $\mathrm{p}_{\mathrm{o}}$ & {$\left[\begin{array}{lll}0 & 0 & 93\end{array}\right]^{\mathrm{T}}$} & - & - \\
\hline $\mathrm{m}_{\mathrm{i}}$ & - & {$\left[\begin{array}{llll}0.012 & 0.012 & 0\end{array}\right]^{\mathrm{T}}$} & {$\left[\begin{array}{lll}0 & 0 & 0\end{array}\right]^{\mathrm{T}}$} \\
\hline
\end{tabular}

Figure A:9 shows the depth measurement standard deviation as a function of change in angle between the two views for the $1 \mathrm{x}$ experiment. Here, the minimum error was $0.01 \mathrm{~cm}$, which required a rotation of $90^{\circ}$. This much rotation is acceptable for objects with axial symmetry, but is 


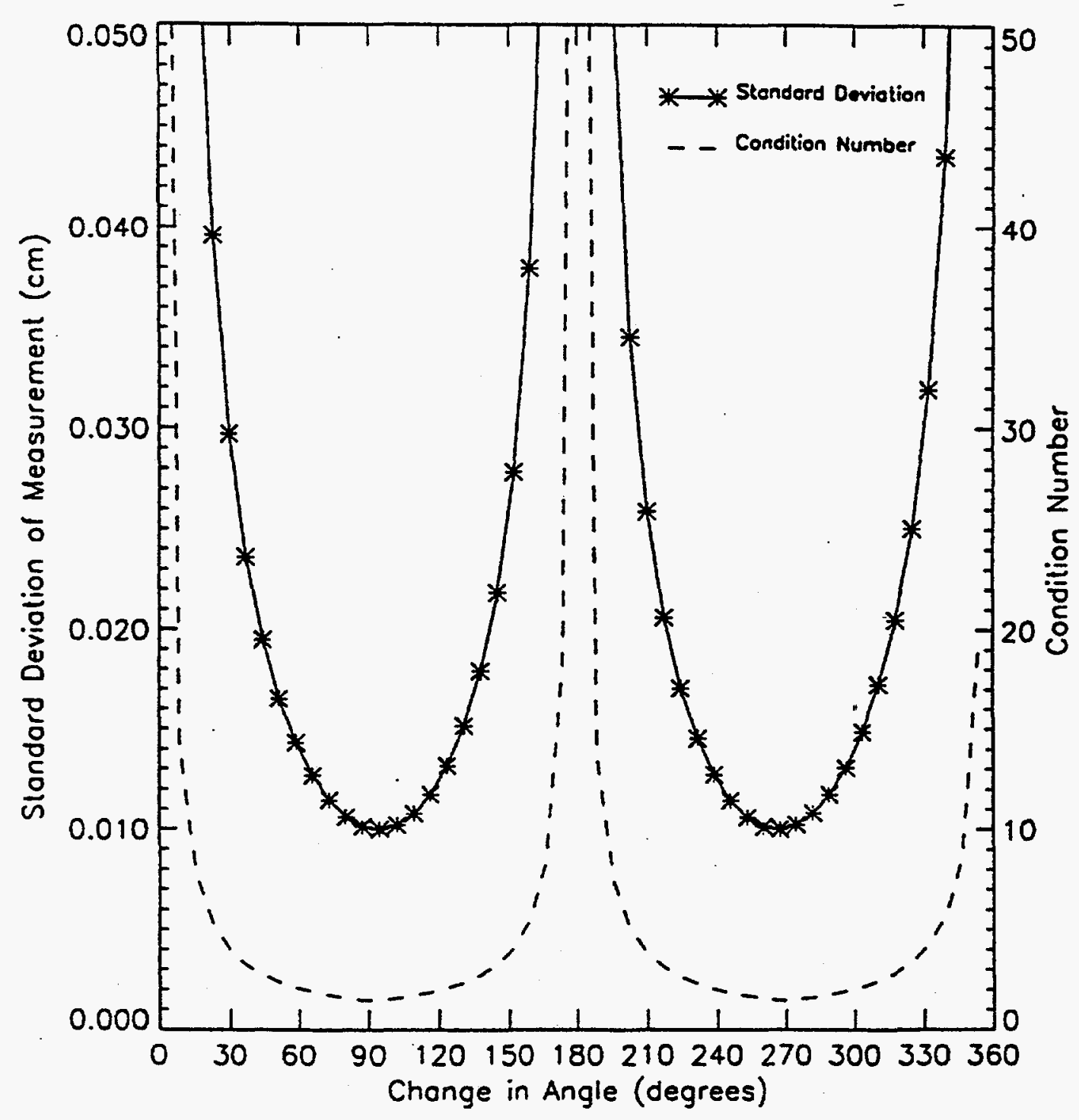

Figure A.9. Two-view rotation experiment, $1 \mathrm{x}$ magnification.

not acceptable for plate-like geometries. Using a more typical rotation of $20-30^{\circ}$ resulted in a factor of 4 to 5 increase in error.

Figure A.10 shows the results for the 20x experiment. The standard deviation of the depth measurement dropped to less than $0.001 \mathrm{~cm}$ at large rotation angles, and was less than $0.01 \mathrm{~cm}$ over the typical range of $5-30^{\circ}$. Again, this much magnification places limits on the sample size, but it is clear that using as high a magnification as possible is beneficial towards reducing measurement errors. The microfocus X-ray source makes magnification practical; however, a standard broad-focus source 


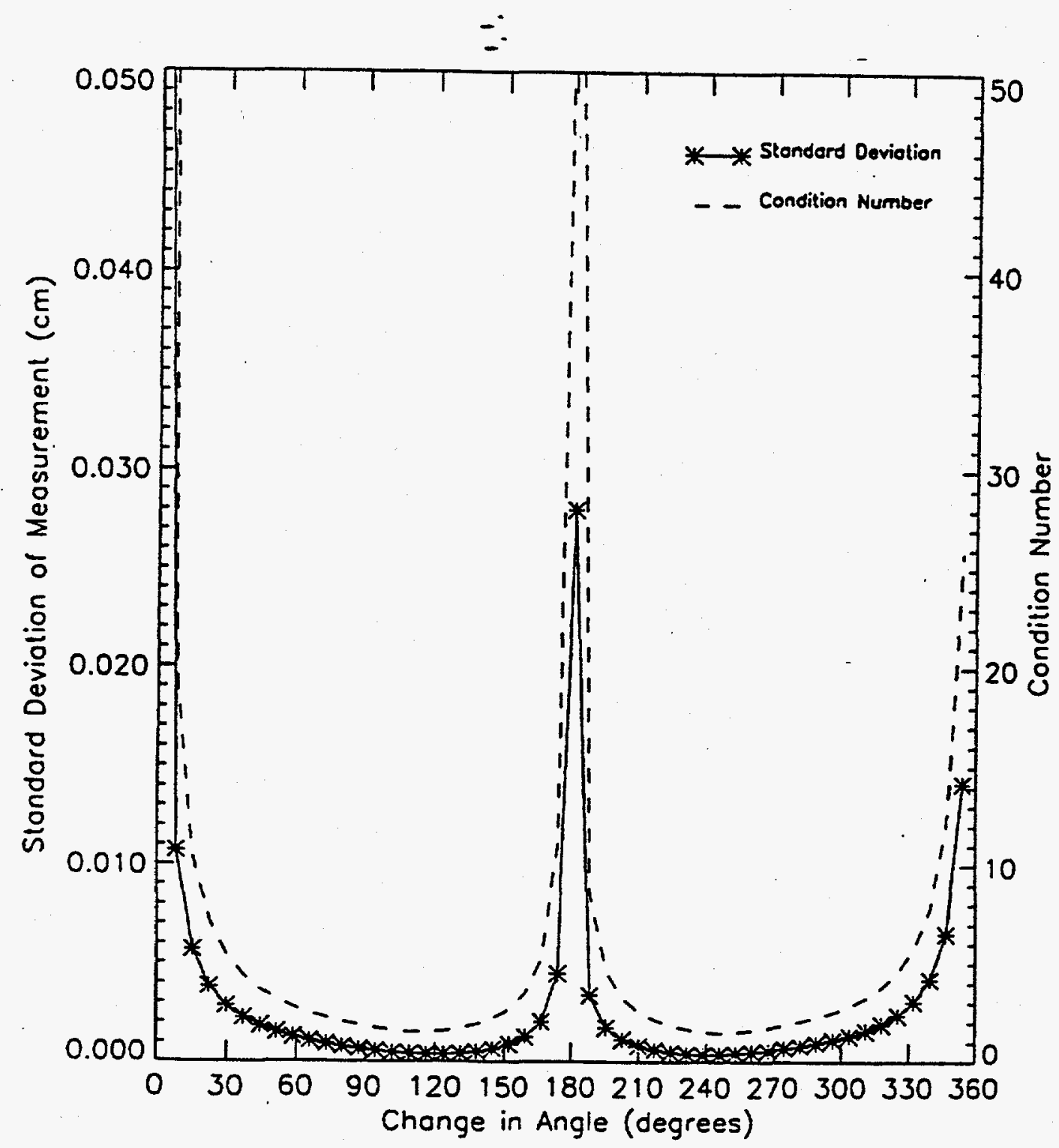

Figure A.10. Two-view rotation experiment, 20x magnification.

can also be used to achieve good results with a $1 \mathrm{x}$ configuration as long as the sample can be rotated a greater amount.

The previous simulations demonstrated the sensitivity to random errors in selecting the feature points for a variety of configurations. The next experiments investigate errors due to mis-measuring the $\mathrm{Z}$ positions of the $\mathrm{X}$-ray source and positioner center of rotation with respect to the detector. Here, random noise is zero on all parameters, and bias is applied to selected quantities. 
Source position bias. The two-view rotation experiment was used as before, except the feature point measurement noise was set to zero. The forward model generated measurement data based on the nominal laboratory setup. The effect of mis-measuring the $Z$ position of the $X$-ray source was simulated by adding a bias to $s_{3}$ and computing the range point estimate using the "incorrect" value. 100 runs were used to develop a distribution of estimates, and the bias was calculated by subtracting the true range point value from the average estimated value. Since changing the sign of the $\mathrm{X}$-ray source $\mathrm{Z}$ bias simply changed the sign of the range point bias, only the results of a positive source bias values will be presented here.

The bias in the range point estimate was found to be a weak function of the rotation angle between the two views. For the $x$ configuration, the bias was $-0.029 \mathrm{~cm}$ at $0^{\circ}$, and $-0.031 \mathrm{~cm}$ at $180^{\circ}$. In between these points, the bias followed a sinusoidal variation with a single period over the range $0-360^{\circ}$. Table A. 6 lists the results obtained using multiple magnifications and source bias values. From this table, it is evident that depth bias is slightly affected by the rotation angle for the two-view experiment, but this effect is down an order of magnitude compared to the effect of error in determining the $\mathrm{Z}$ position of the source. The magnitude of the bias is negligible for $1 \mathrm{x}$ magnification, and increases steadily as magnification increases. For the $4 \mathrm{x}$ configuration, the magnitude of the bias is limited to $0.01-0.02 \mathrm{~cm}$ for an error of $0.5 \mathrm{~cm}$. This provides an upper limit on the bias error, since the tape measure accuracy lies between $0.1-0.2 \mathrm{~cm}$.

Center of rotation position bias. A similar experiment was performed to determine sensitivity to bias error in determining the positioner center of rotation $Z$ position; Table $A .7$ tabulates the results. In this case, a positive bias in $c_{3}$ resulted in a $1: 1$ effect plus some residual positive bias. For example, at $4 x$ magnification, a $0.5 \mathrm{~cm}$ measurement error resulted in $0.52 \mathrm{~cm}$ error in the range point estimate; the 1:1 effect is $0.02 \mathrm{~cm}$ and the residual bias is $0.02 \mathrm{~cm}$. Consequently, even small errors in determining the center of rotation are reflected directly in the result. This is a significant problem when absolute measurements are to be made in the global coordinate system. However, most measurements are made relative to the boundaries of the sample, so measurements in the local coordinate system are only subject to the residual bias effects of $0.01-0.0: 3 \mathrm{~cm}$ for bias errors of $0.1-0.5 \mathrm{~cm}$. 


\begin{tabular}{|c|c|c|}
\hline Table A.6. Sensitivity to bias in X-ray source Z position measurement. \\
\hline $\begin{array}{c}\text { Source Z Bias } \\
(\mathrm{cm})\end{array}$ & $\begin{array}{c}\text { Minimum Bias } \\
(\mathrm{cm})\end{array}$ & $\begin{array}{c}\text { Maximum Bias } \\
\text { (cm) }\end{array}$ \\
\hline 1x Magnification & & \\
\hline 0.1 & +0.000 & +0.000 \\
\hline 0.5 & +0.000 & -0.001 \\
\hline 1.0 & +0.000 & -0.001 \\
\hline 2.0 & +0.000 & -0.002 \\
\hline $4 \times$ Magnification & & -0.003 \\
\hline 0.1 & -0.003 & -0.015 \\
\hline 0.5 & -0.014 & -0.031 \\
\hline 1.0 & -0.029 & -0.061 \\
\hline 2.0 & -0.057 & \\
\hline $20 \times$ Magnification & & -0.039 \\
\hline 0.1 & -0.037 & -0.193 \\
\hline 0.5 & -0.185 & -0.986 \\
\hline 1.0 & -0.367 & -0.767 \\
\hline 2.0 & -0.724 & \\
\hline
\end{tabular}

\section{Experiment: Automotive Air Conditioner Part}

This sample is an aluminum casting used as part of an automobile air conditioner. A drawing of a CAD model for this part is shown in Figure A.11. The part has a wide variety of thickness variations, with the thickest sections on either end of the part, and the thinnest regions in the middle of the part. These thickness variations result in a large variation of $\mathrm{x}$-ray absorption, and leads to simultaneous underexposure (not enough photons for good signal-to-noise ratio) and overexposure (detector saturation) in different parts of the image. The real-time system has an advantage in that the $\mathrm{X}$-ray source power and image processing parameters can by dynamically altered to optimize different parts of the image, based on which region is of interest at the moment. 
Table A.7. Sensitivity to bias in sample center rotation $\mathrm{Z}$ position measurement.

\begin{tabular}{|c|c|c|}
\hline $\begin{array}{c}\text { Source } Z \text { Bias } \\
(\mathrm{cm})\end{array}$ & $\begin{array}{c}\text { Minimum Bias } \\
(\mathrm{cm})\end{array}$ & $\begin{array}{c}\text { Maximum Bias } \\
(\mathrm{cm})\end{array}$ \\
\hline $1 \times$ Magnification & & 0.10 \\
\hline 0.1 & 0.10 & 0.51 \\
\hline 0.5 & 0.51 & 1.02 \\
\hline 1.0 & 1.02 & 2.04 \\
\hline 2.0 & 2.04 & \\
\hline $4 \times$ Magnification & & 0.11 \\
\hline 0.1 & 0.10 & 0.53 \\
\hline 0.5 & 0.52 & 1.05 \\
\hline 1.0 & 1.05 & 2.10 \\
\hline 2.0 & 2.10 & \\
\hline $20 \times$ Magnification & & 0.14 \\
\hline 0.1 & 0.14 & 0.70 \\
\hline 0.5 & 0.70 & 1.40 \\
\hline 1.0 & 1.40 & 2.80 \\
\hline 2.0 & 2.80 & \\
\hline
\end{tabular}

This sample contains cracks due to shrinkage porosity throughout its interior due to improper manufacture. A particular fork-shaped crack located near the "3" was selected for measurement; the real-time image of this feature is indicated in Figure A.12. The gray curved stripe on the left of the image is the projection of the center fillet of the casting to the left of the " 3 ." The fillet appears curved since the casting was initially rotated about $+20^{\circ}$ to make the flaw more visible from the dark patch on the right side of the image. A lead marker was affixed to the back surface of the part which was placed closest to the detector. Five range points were selected to characterize the flaw, and two points were used to identify the inner and outer surface boundaries. These features are indicated as an overlay in Figure A.12. The middle of the " 3 " was used as the inner surface feature, and the lead 


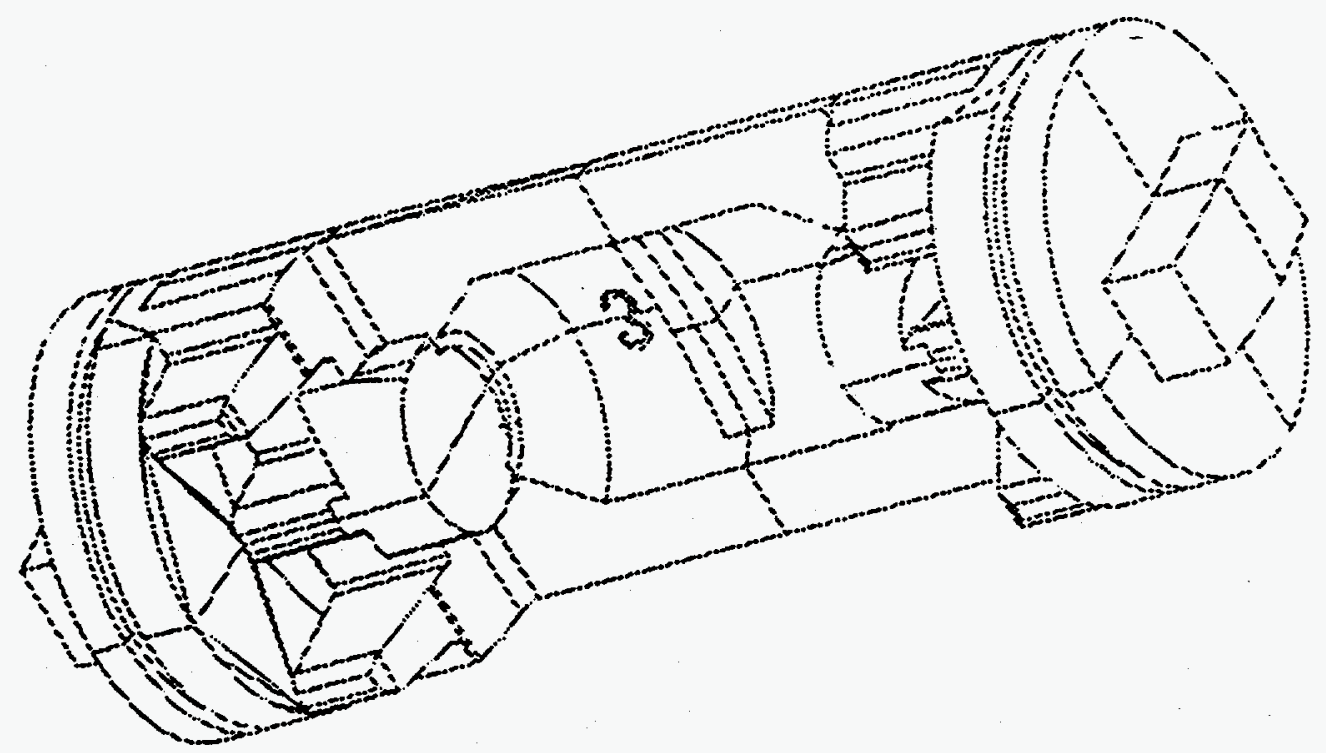

Figure A.11. CAD model of automobile air conditioner part.

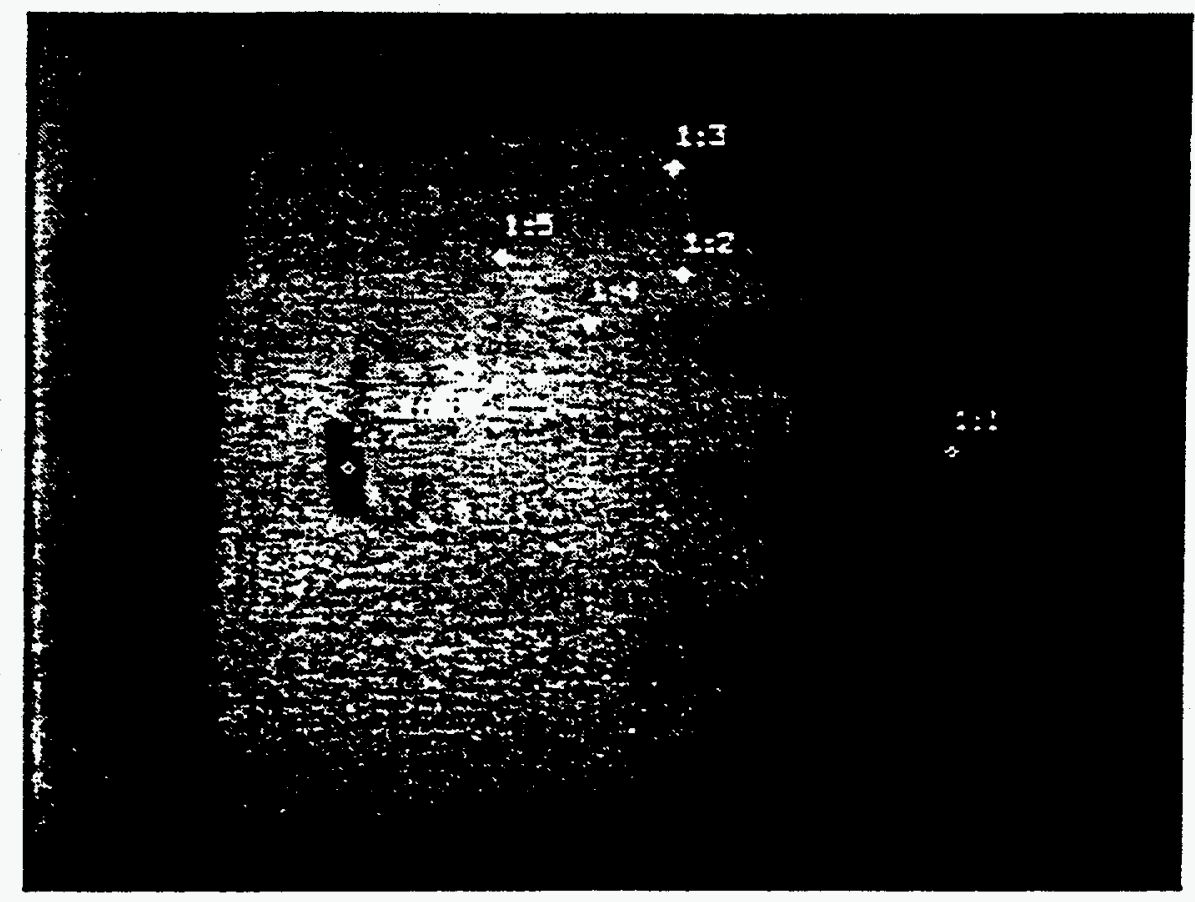

Figure A.12. Real-time image of image of air conditioner part. 
marker was used as the outer surface feature. This demonstrates how including recognizable featuresin the design of the part can eliminate the need to attach external markers to identify the surface boundaries.

The results of three complete measurements are plotted in Figure A.13. Since only two markers were used to locate the surface boundaries, the curved contours of the part were not measured. Dotted lines were drawn through the surface mark locations to give an idea of the flaw orientation relative to the surface. As mentioned previously, the part had an initial rotation of $+20^{\circ}$; this angle was used to plot the surface boundaries in the top view ( $X Z$ plane). Points 2 and 4 of the third data set had a depth deviation of $0.05 \mathrm{~cm}$ compared to the other two data sets, which accounts for the "messiness" of the plot in the side view (YZ plane). The flaw boundaries for these points were not as well-defined, so the feature point selection had higher variance. Furthermore, it was difficult to identify the corresponding points since the flaw significantly changed shape between views. This demonstrates how flaw morphology plays a significant role in determining screen measurement variance.

MEM processing was used to sharpen the flaw features located within two $256 \times 256$ subimages (Figure A.14); the flaw boundaries were only marginally enhanced. Three complete measurements were made again using the MEM processed images, with the results plotted in Figure A.15. The $X$ and $Y$ locations for all except Point 2 were determined with less variation, but the depth variation was not reduced compared to the previous measurement. The processing time for a 256 x 256 image was 4 minutes on the DEC5000. The improvement due to MEM processing was marginal as far as image quality is concerned, and was negligible in terms of reducing operator error in determining the feature points. Thus, the long computation time of MEM was not found to be justified.

This sample demonstrated the ability to make a 3-D crack orientation and size measurements. The features of interest had the poorest definition of the four samples used in this chapter, and the results showed an increased variance in depth as a result. Use of an edge detection scheme would eliminate the operator ambiguity problem, yet these features would also present a challenge to an 

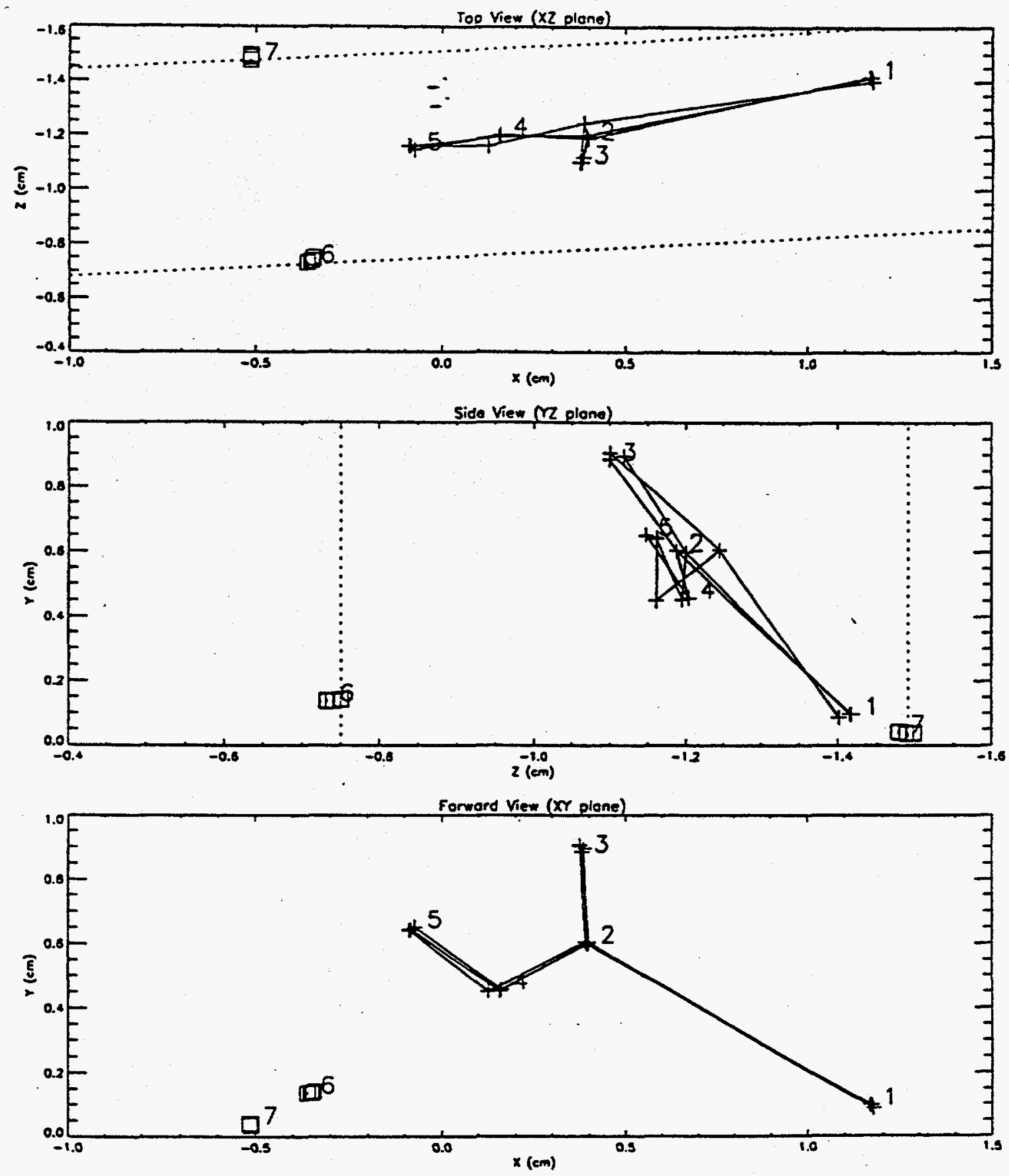

Figure A.13. Range point measurements for air conditioner part. 


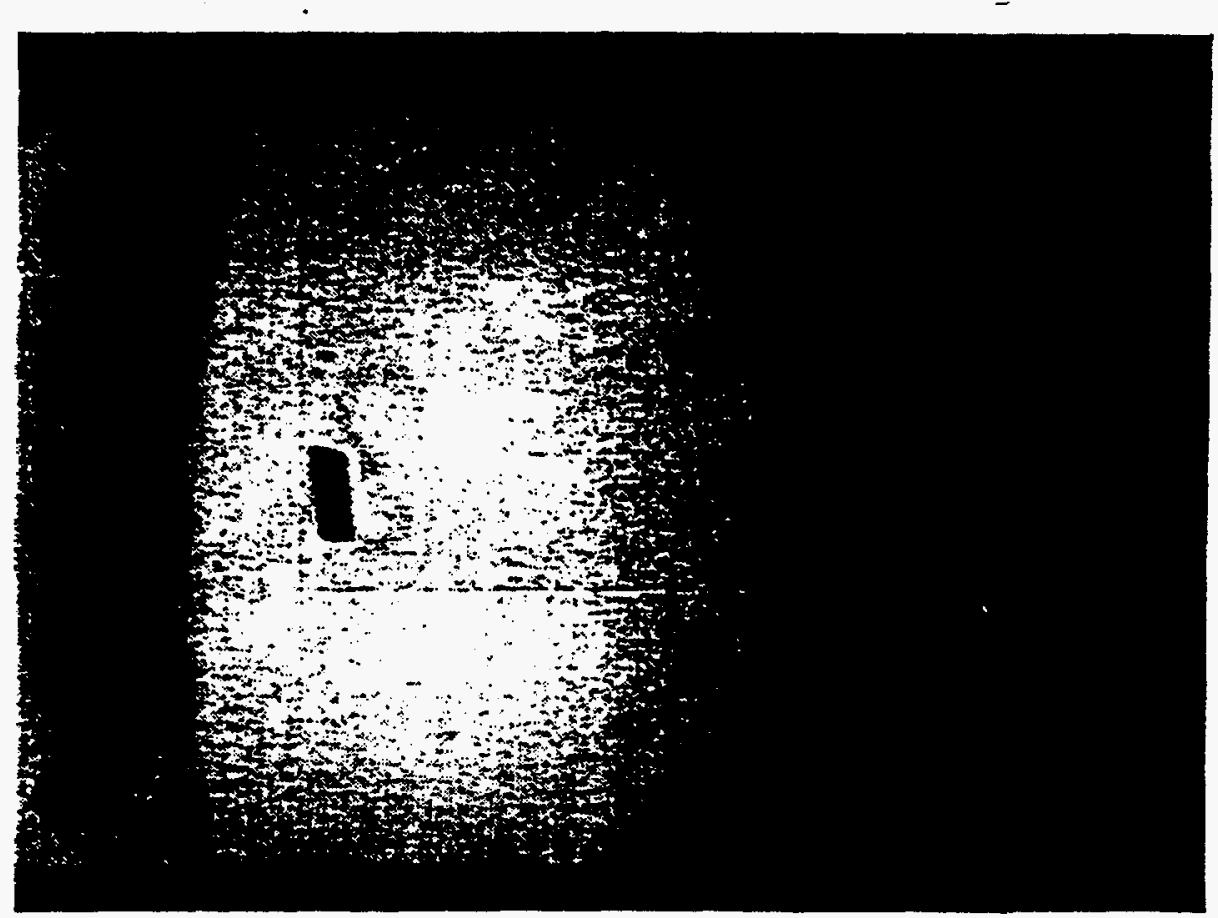

Figure A.14. MEM processed image of air conditioner part.

automated edge detector as well. This showed that measurement error in determining the feature points is a significant component of the overall measurement error, so it would be valuable to incorporate as much automatic and robust edge detection as possible. 

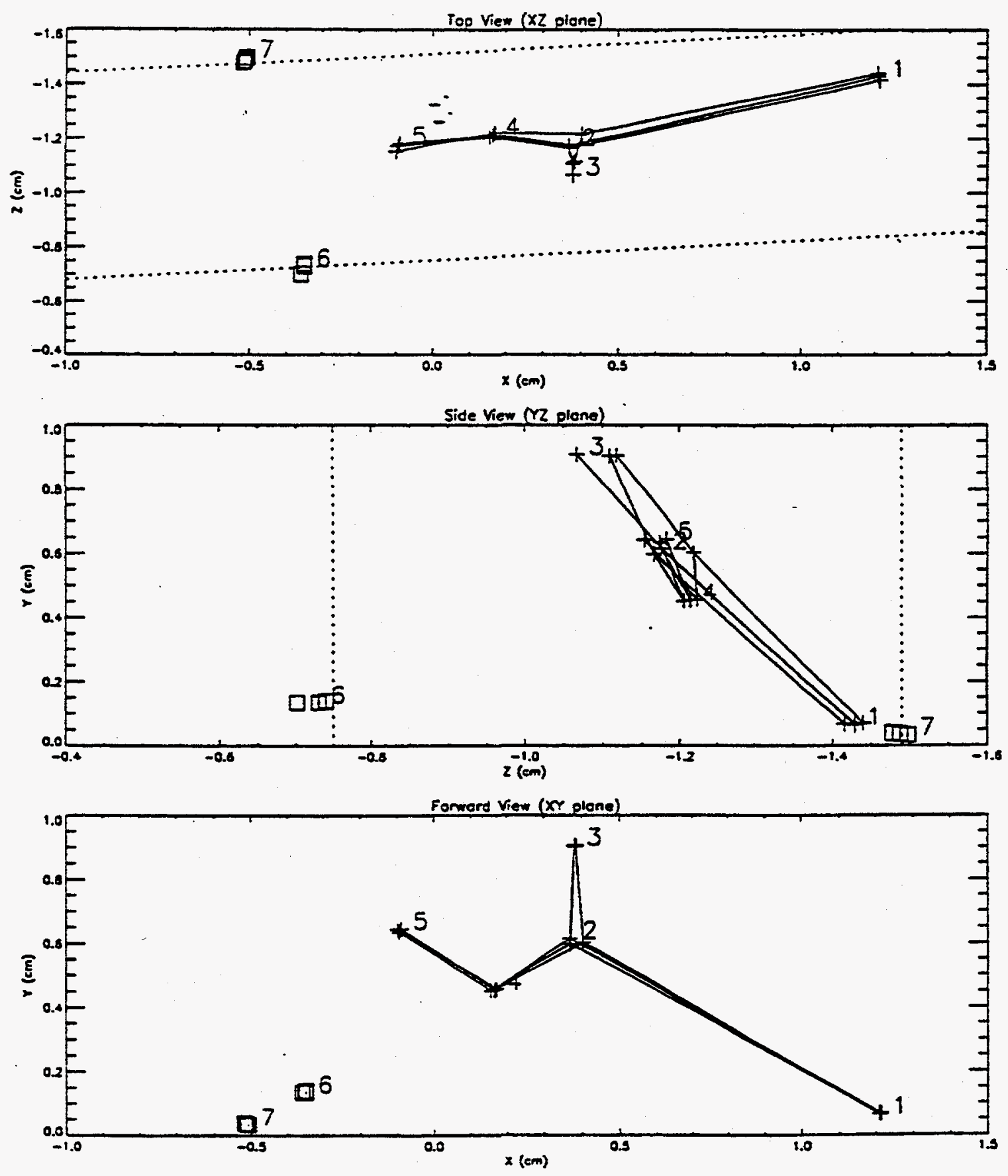

Figure A.15. Range point measurements for air conditioner sample using MEM processed image. 
APPENDIX B

EXPERIMENTAL RESULTS 


\section{APPENDIX B EXPERIMENTAL RESULTS}

The experimental results obtained during the course of this research are presented, and the feasibility of using stereography to obtain $3 \mathrm{D}$ co-ordinates of feature points embedded within samples is demonstrated. This experimental inspection method was tested on three samples moving gradually from the simple to the complex. The results were collaborated with the measurements done using other methods. The first sample used was fabricated in the lab. It is a couple of wires sandwitched between two aluminum plates. The second example is an aluminum test sample in the form of a rod. Its a casting which has porosity caused due to solidification defect.

\section{Experimental Setup}

The X-ray source, sample positioner, and detector are to be properly aligned. The global coordinate system is centered on the image intensifier screen. The X-ray source is taken to be present in the positive $z$ plane. The sample co-ordinate system is defined on the sample. The $x, y$, and $z$ coordinates are output relative to the global co-ordinate system. It is the responsibility of the operator to convert the global co-ordinates of the feature points to another system with origin at one of the selected feature points. This has the advantage that the operator can select the feature point relative to which he wants the distances and orientation calculated.

\section{X-ray Source Calibration}

The purpose of this calibration is to calculate the distance from the detector plane to the X-ray source. Though the tape measure is not altogether done with, we will measuring smaller distances than earlier with it thereby reducing the error introduced. A rudimentary artifact was designed, a cube $0.25 \times 0.25 \times 0.25$ inch. It is in the initial view, attached to the detector plane so that we get a $1 \mathrm{X}$ magnification. It is then moved back till a $2 \mathrm{x}$ magnification is obtained. The magnification can be obtained by measuring the pixel values of an edge of the cube. The artifact is then moved by a distance $\mathrm{p}$ to get a $3 \mathrm{x}$ magnification. Six times the distance $\mathrm{p}$ gives the source to detector distance.

As shown in the Figure B.1 


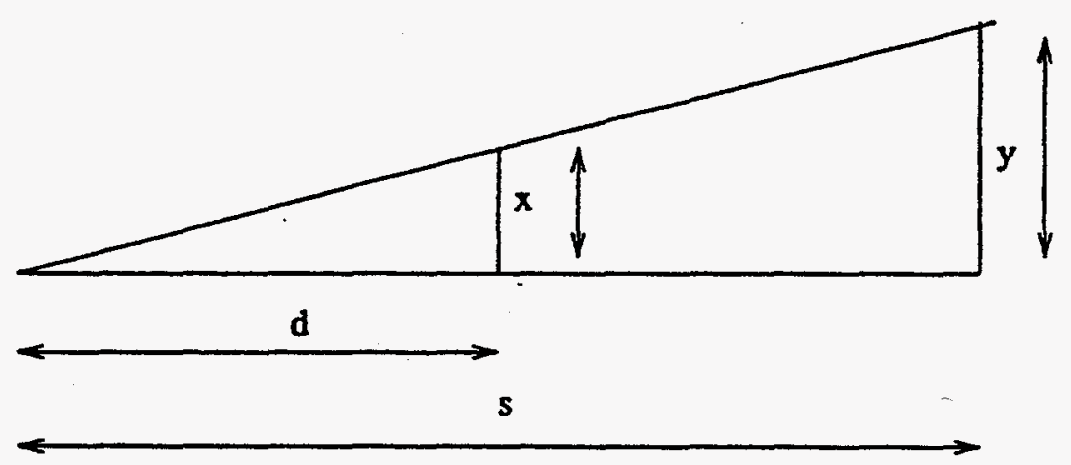

Figure B.1. X-ray source calibration.

$$
\text { for } 1 X \quad d=s
$$

$$
\text { for } 2 X \quad d=s / 2
$$

$$
\text { for } 3 X \quad d=s / 3
$$

by considering the Equations B.1 and B.2 we have

$$
p=s / 6
$$

So 6 times p gives the source to detector distance. Hence the $\mathrm{X}$-ray to source distance is calibrated. The size of the artifact is restricted by the scope of the view of the image intensifier.

\section{Experiment: Aluminum Plates With Embedded Lead Wires}

A fabricated sample is used for this experiment. The sample is a couple of aluminum plates bunched together with lead wires placed between them as shown in the Figure B.2. The objective of the experiment was to calculate the length of the embedded wires and to compare it with visual 


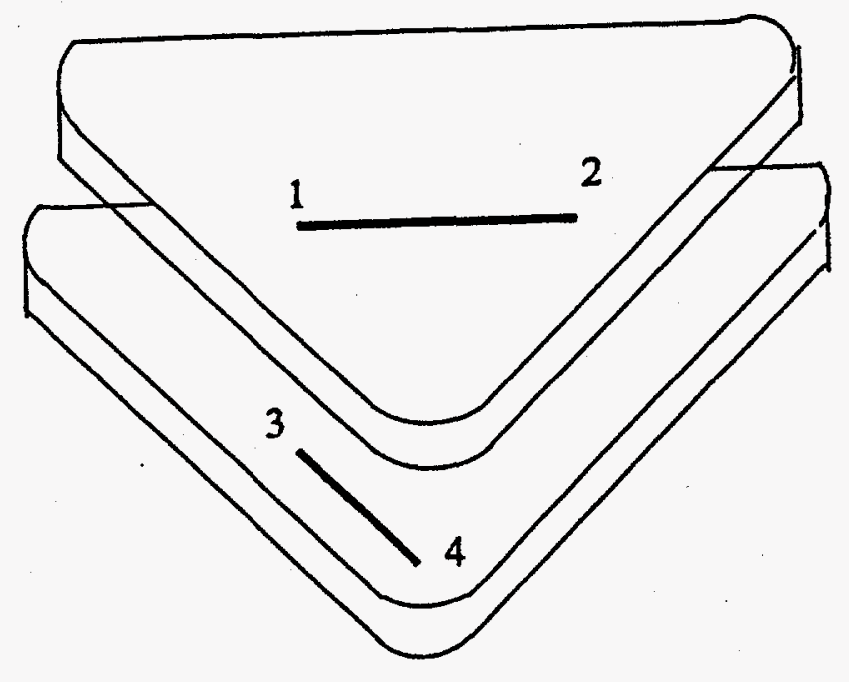

Figure B.2. Sample 1 .

measurement. The depth of the wires was also measured and the result was collaborated with visual inspection. The X-ray generator was operated at $60 \mathrm{KV}$ and $600 \mu \mathrm{A}$. After the initial views the sample was rotated and a decrease in the intensity of the image was observed. This was because of the increased effective thickness of the plates. This makes the selection of feature points difficult, introducing errors into the system. Detectability is characterized by percentage thickness of the feature relative to the host material. The real time detector has the capability to detect $2 \%$ thickness while film can detect up to $1 \%$ (8). Therefore for a given flaw thickness, there exists a maximum rotation angle less than 90 degrees that can still detect the flaw.

For the second orientation the KV value was boosted up to $80 \mathrm{KV}$. Fifty frames were grabbed to remove the blurring effect present. The same experiment was repeated for various angles of rotation and by observing the error results it was found that an angular shift of $\mathbf{4 5}$ degrees gave the least error. From the Table B.1 it is seen that the error that occurred is about $0.1 \mathrm{~mm}$ between the visual length and experimentally measured lengths.

Hence it has been demonstrated in this experiment that stereography can generate the co-ordinates of a limited number of points within a sample. This demonstrates the feasibility of stereography. 


\begin{tabular}{|c|c|c|c|}
\hline \hline Table B.1. Length of wires. & $\mathrm{x}$ & $\mathrm{y}$ & $\mathrm{z}$ \\
\hline Point & 0 & 0 & 0 \\
\hline Point 1 & 1.215 & 0.20 & 0 \\
\hline Point 2 & 0 & 0 & 0 \\
\hline Point 3 & 0.10 & 0.716 & 0 \\
\hline Point 4 & & & \\
\hline \multicolumn{5}{|c|}{ Error in lengths } & \\
\hline \multicolumn{5}{|c|}{} & Visual & Experimental & - \\
\hline Wire & 1.215 & 1.220 & - \\
\hline Wire 1-2 & 0.710 & 0.716 & - \\
\hline Wire 3-1 & &
\end{tabular}

\section{Experiment: Sample Aluminum Casting Bar}

The sample in this case is an experimental casting as shown in the Figure B.3. This sample has porosity due to variation in the cooling temperatures of the molten metal poured into the mould.

The bar did not have a well defined point that could be used as a reference point near the ROI. Lead wires were placed on the edge of the sample so that the flaw could be characterized with respect to the bar boundary. Though the bar had uniform thickness, $I$ had to increase the $\mathrm{x}$-ray intensity to view the flaw properly after the bar was rotated at an angle of 45 degrees. Also because of the limited field of view of the detector I could not view the whole sample at a time so, a ROI was selected and image processing done on that ROI to view the flaw clearly.

There were a couple of flaws that I could see using RTR. But, I selected a flaw that was predominant and it was in the middle of the thickness. Two views of the sample were taken. It was observed that there was a porosity along the length of the bar and the ends of it were at different depths so it had orientation as well though very small. The results are tabulated in the Table B.2. 

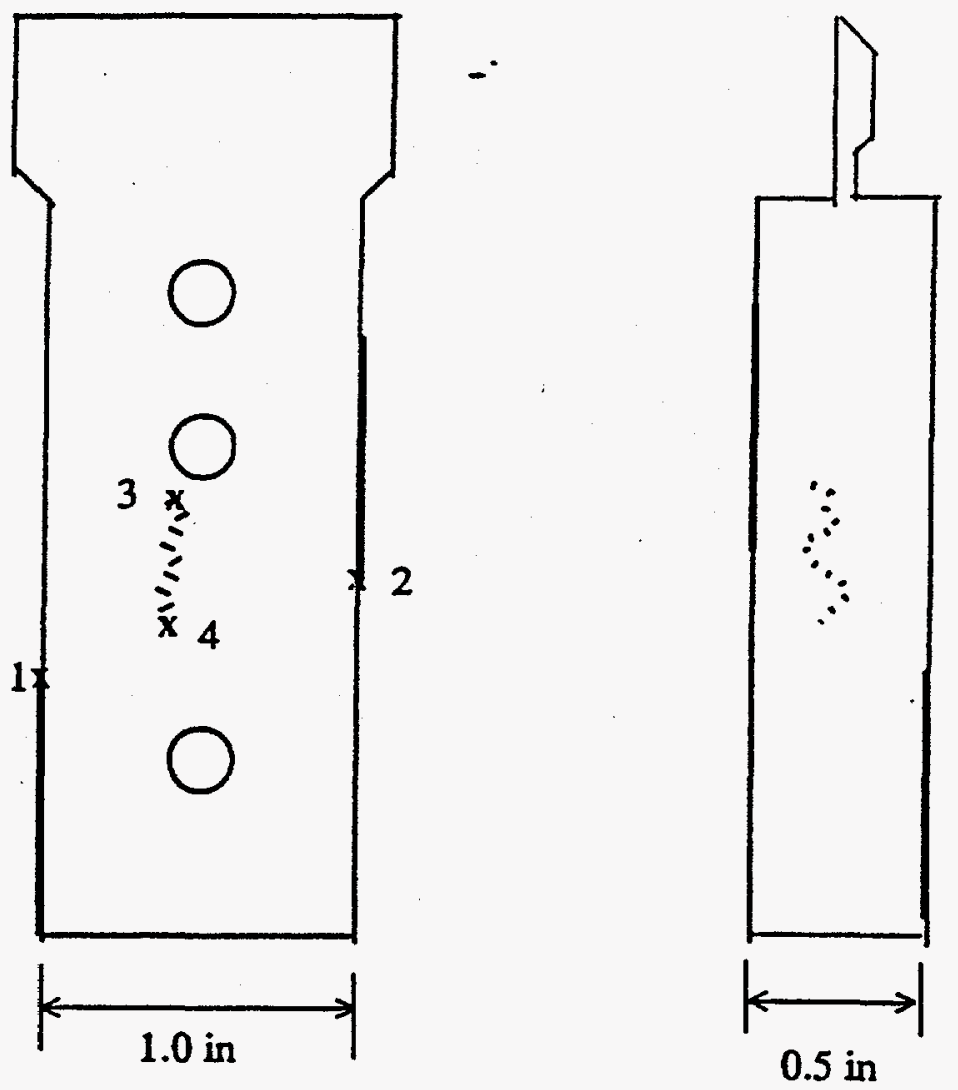

Figure B.3. Test Casting.

Table B.2. Porosity length in aluminum bar.

\begin{tabular}{|c|c|c|}
\hline Point & $x$ & $y$ \\
\hline Reference 1 & 0 & 0 \\
\hline Porosity 1 & 0.43 & 0.25 \\
\hline Porosity 2 & 0.51 & 0.26 \\
\hline
\end{tabular}

The results were collaborated by looking at a radiograph of the sample, and I found a match between the dimensions. From this experiment it can be observed that samples with regular geometries having flaws can be more easily detected than those with irregular geometries. Though I used lead markers on the sample it will be better if we had built in markers on the part handler. 


\section{BIBLIOGRAPHY}

[1] Martin, G. J., and Blessings, K. V., High resolution CCD camera for industrial imaging, SPIE, 1993.

[2] Kaelble, F. E., Handbook of x-rays, McGrawhill Book Company, 1993.

[3] Operation and Maintenance Manual IRT64050-A01, IRT Industrial X-ray Systems, 3030 Callan Road, San Diego, CA, 1990.

[4] Operating Instructions Manual, FeinFocus X-ray units, Leybold Inc., San Jose, CA, 1990.

[5] WINSOCK Reference Manual, Public Domain MicroSoft Windows Socket Library, http://www.microsoft.com, 1995.

[6] DT2867 Hardware \& Software Manuals, Data Translation Inc., Marlboro, MA, 1991.

[7] Stevens, R. W., UNIX Network Programming, Prentice Hall, Englewood Cliffs, NJ, 1990.

[8] Doering, E. R., Ph.D Thesis, Department of Electrical Engineering, Iowa State University, Ames, IA, 1992.

[9] Wallingford, R. M., Ph.D Thesis, Department of Electrical Engineering, Iowa State University, Ames, IA, 1990.

[10] Press, W., and Flannery, B. P., and Teukolsky, S. A., Numerical Recipes in C - The art of scientific computing, Cambridge, U.K., 1988.

[11] Quinn, R. A., and Sigl, C. C., Radiography in Modern Industry, Eastman Kodak, Rochester, NY, 1980.

[12] Veress, S. A., X-ray Photogrammetry Systems and Applications, 1989.

[13] Bryant, L. E., Non-Destructive Testing Handbook, Second Edition, Vol. 3, American Society for Non-Destructive Testing, Columbus, OH, 1985.

[14] Petzold, C., Programming Windows 3.1, Microsoft Press, 1992.

[15] Stallings, W., Data in Computer Communications, 1991.

[16] WINSOCKET 1.1 HELP, http://www.stardust.com, 1995. 